\title{
Brain sterol metabolism : modulating Alzheimer's disease
}

Citation for published version (APA):

Vanmierlo, T. (2010). Brain sterol metabolism : modulating Alzheimer's disease. [Doctoral Thesis, Maastricht University]. Maastricht University. https://doi.org/10.26481/dis.20100930tv

Document status and date:

Published: 01/01/2010

DOI:

10.26481/dis.20100930tv

Document Version:

Publisher's PDF, also known as Version of record

\section{Please check the document version of this publication:}

- A submitted manuscript is the version of the article upon submission and before peer-review. There can be important differences between the submitted version and the official published version of record.

People interested in the research are advised to contact the author for the final version of the publication, or visit the DOI to the publisher's website.

- The final author version and the galley proof are versions of the publication after peer review.

- The final published version features the final layout of the paper including the volume, issue and page numbers.

Link to publication

\footnotetext{
General rights rights.

- You may freely distribute the URL identifying the publication in the public portal. please follow below link for the End User Agreement:

www.umlib.nl/taverne-license

Take down policy

If you believe that this document breaches copyright please contact us at:

repository@maastrichtuniversity.nl

providing details and we will investigate your claim.
}

Copyright and moral rights for the publications made accessible in the public portal are retained by the authors and/or other copyright owners and it is a condition of accessing publications that users recognise and abide by the legal requirements associated with these

- Users may download and print one copy of any publication from the public portal for the purpose of private study or research.

- You may not further distribute the material or use it for any profit-making activity or commercial gain

If the publication is distributed under the terms of Article $25 \mathrm{fa}$ of the Dutch Copyright Act, indicated by the "Taverne" license above, 


\section{Brain Sterol Metabolism - Modulating Alzheimer's Disease}

Tim Vanmierlo 
Brain Sterol Metabolism - Modulating Alzheimer's Disease Tim Vanmierlo

ISBN: 978-90-6464-411-5

Cover design by Silke Reumers

Printed by GVO drukkers \& vormgevers B.V.| Ponsen \& Looijen (C) Tim Vanmierlo, Maastricht 2010 


\section{Brain Sterol Metabolism - Modulating Alzheimer's Disease}

\section{Proefschrift}

Ter verkrijging van de graad van doctor

aan de Universiteit Maastricht, op gezag van de Rector Magnificus, Prof. mr. G.P.M.F. Mols

volgens het besluit van het college van Decanen, in het openbaar te verdedigen

op donderdag 30 september 2010 om 12.00 uur.

door

Tim Vanmierlo

Geboren te Neerpelt op 17 mei 1983 
PROMOTOR

Prof. Dr. Harry W. M. Steinbusch

COPROMOTORES

Dr. Monique Mulder (Erasmus MC, Rotterdam)

Prof. Dr. Dr. rer. nat. Dieter Lütjohann (Universitätsklinikum Bonn, Duitsland)

BEOORDELINGSCOMMISSIE

Prof. Dr. Jim van Os (voorzitter)

Dr. Arjan Blokland

Prof. Dr. Folkert Kuipers (UMCG Groningen)

Dr. Jogchum Plat

Prof. Dr. Jochen Walter (Universitätsklinikum Bonn, Duitsland)

The studies described in this thesis were supported by a Marie Curie early stage training site fellowship grant (MEST-CT-2005-02058)

Financial supports for the publication of this thesis by "Eli Lilly Nederland", "Danone Research - Centre for Specialised Nutrition" and the "International Stichting Alzheimer Onderzoek (ISAO)" are gratefully acknowledged. 


\section{Contents}

\section{CHAPTER I}

GENERAL INTRODUCTION

\section{CHAPTER II}

Alterations In BRAIN CHOLESTEROL METABOLISM IN THE

APPSLXPS1MUT MOUSE, A MODEL FOR ALZHEIMER'S DISEASE.

\section{CHAPTER III}

LIVER X RECEPTOR ACTIVATION RESTORES MEMORY IN AGED AD MICE

WITHOUT REDUCING AMYLOID

\section{CHAPTER IV}

IRREVERSIBLE ACCUMULATION OF PLANT STEROLS IN THE BRAIN

\section{CHAPTER V}

A Study on the Role of Cerebral Plant Sterol Accumulation on

MEMORY AND ANXIETy RELATED BEHAVIOR IN ABCG5-/- MICE

\section{CHAPTER VI}

The Plant Sterol Brassicasterol as Biomarker in Alzheimer's Disease

\section{CHAPTER VII}

GENERAL DisCUSSION

SUMMARY / SAMENVATTING

DANKWOORD 



\section{Chapter I}

\section{General Introduction}

Part of this chapter is based on: Lütjohann D., Vanmierlo T. and Mulder M. (2009) Cholesterol trafficking in the brain, in Ehnholm C. (ed) Cellular lipid metabolism, Berlin: Springer Berlin Heidelberg 131-155 (2009) 


\section{Alzheimer's Disease}

\section{Origin of "Alzheimer"s Disease"}

Alzheimer's disease (AD) is the most common form of elderly dementia. In 2006, approximately 26.6 million people world wide suffered from AD. Mainly due to the increased aging of the world population, over 100 million people will be suffering from the disease by the year 2050, corresponding to 1 in 85 persons. $40 \%$ of the AD patients will require intensive nursing home care, which will lead to a massive economic burden on social health care resources $[1,2]$.

$\mathrm{AD}$ was first presented as case report at the $37^{\text {th }}$ Assembly of the Society of Southwest German Psychiatrists by Alois Alzheimer on November 4, 1906 in Tübingen. The case report concerned a disease course of $4 \frac{1}{2}$ years in a 55 years old female patient (Auguste Deter) investigated by Alois Alzheimer and Emil Kraepelin. In contrast to the title of this paragraph, Alzheimer did not have the disease himself, but he first described the pathology which was later named after him. The course of the disease was featured by deteriorating memory, speaking, physical and social abilities. At the end, Auguste Deter was completely apathetic, lying on her bed with retracted legs, incontinent and had decubitus ulcers, despite good nursing. The autopsy revealed a uniformly atrophic brain without any macroscopic abnormalities. Yet, further investigations revealed that the larger cerebral vessels showed atherosclerotic changes. Using Bielschowsky's silver staining, remarkable several staged neurofibrillary changes were observed. In normal appearing cells, first one or a few fibrils occurred, which were prominent because of their thickness and their unusual impregnation. Then, the fibrils coalesced into dense bundles and gradually progressed to the surface of the cell. Finally, the nucleus and the cell degenerated and only a tangled bundle of fibrils outlined the locus where the nerve cell was situated. About a quarter of all neurons in the cerebral cortex displayed these changes and especially the upper cell layers of the cortex neurons had entirely disappeared. In addition, distributed over the entire cortex, especially numerous in the upper layers, small foci were visible even without staining. These were due to the storage of a peculiar substance in the nerve cell in the cortex. It wasn't until 1910, in the chapter "Senile and Pre-senile Dementias" of Kraepelin's "Textbook of Psychiatry", that the term "Alzheimer's disease" was first introduced [3]. Since average life expectancy was around 50 years at the time AD was discovered, dementia was a rather rare disease. Moreover, AD was considered to be a pre-senile form of dementia until Tomlinson and coworkers (1968-1970) described that the dementia, from which the majority of the elderly subjects suffered, was indeed the form of dementia described by Alzheimer and Kraepelin in 1906 [4, 5]. From then on, 
$\mathrm{AD}$ was no longer seen as a pre-senile form of dementia, but as a major form of dementia in the elderly population [6]. Today, AD is the most common form of elderly dementia.

\section{Hypothesis on Etiology of Alzheimer's Disease}

Aging is the major risk factor for $\mathrm{AD}$, reflected in an increasing prevalence with advanced age. Less than $1 \%$ of the people between 60-64 years have Alzheimer's disease, whereas $40 \%$ of people older than 85 years are affected [7-9]. The cut-off between early onset (EOAD) and late onset $\mathrm{AD}$ (LOAD) is arbitrarily set at 60-65 years. EOAD and LOAD are clinically indistinguishable, however, EOAD is generally more severe with a more rapid rate of progression, as clinical practice indicates [10]. A family history in EOAD is the second most important risk factor for $\mathrm{AD}$ [11, 12]. Several mutations in the amyloid precursor protein (APP) on chromosome 21, presenilin 1 (PS1) on chromosome 12 and PS2 on chromosome 1, have shown to be autosomal-dominant inheritable [11]. These mutations, eventually all lead to an increased production of amyloid- $\beta$ peptide $(A \beta)$. However, only $10 \%-40 \%$ of the EOAD is believed to be due to inheritable mutations involved in the $A \beta$ generation metabolism, called familial Alzheimer's disease (FAD), equivalent to $1 \%$ of al $\mathrm{AD}$ cases. The larger part of AD cases are not based on direct genetic inheritance of sole risk factors $\mathrm{LOAD}(95 \%)$. Therefore, most $\mathrm{AD}$ cases have a more complex etiology, called sporadic $\mathrm{AD}[7,13]$. Besides the causative genetic mutations in $\mathrm{FAD}$, a large number of environmental and genetic risk factors are known to be involved in the development of sporadic $\mathrm{AD}$ (both in EOAD and LOAD), including aging, hypertension, smoking, overweight, plasma cholesterol, atherosclerosis, etc., as summarized by Hooijmans et al [8]. The most persistent risk factor in AD is the presence of the $\varepsilon 4$ allele of APOE, attributing an important role to the peripheral and central cholesterol metabolism in AD [14].

Several genetic and environmental risk factors are described to be related to the known anatomical pathology underlying dementia in $\mathrm{AD}$, including senile plaques, neuronal tangles, neuronal loss, gliosis, synapse loss, and brain atrophy. Together, functional, anatomical and epidemiological studies have contributed to several important hypothesises on the etiology of $\mathrm{AD}$. 


\section{The Amyloid- $\beta$ hypothesis}

Genetic risk factors of AD include mutations in APP, PS1, and PS2, trisomy 21, and ApoE4. Most of these risk factors are related to changes in the $A \beta$ metabolism and result in an elevated $A \beta$ plaque deposition. Therefore, $A \beta$ is assumed to be central in the $\mathrm{AD}$ disease process [15]. The role of $\mathrm{A} \beta$ in $\mathrm{AD}$ is referred to as "the $\mathrm{A} \beta$ hypothesis".

APP is a glycosylated transmembrane spanning type-I protein, consisting of 770,751 or 695 amino acids (aa). Neurons mainly express the APP695 [16], lacking the Nterminal Kunitz protease inhibitor (KPI) domain. $A \beta$ is generated by alternative cleavage of APP as depicted in figure 1. In general APP695 is spliced by the $\alpha$ secretase complex, into the soluble $\operatorname{APP} \alpha(\operatorname{sAPP} \alpha)$ and the membrane resident $\mathrm{C} 83$ peptide between aa687 and aa688. The C83 peptide is subsequently cleaved by the $\gamma$ secretase complex into the p3 fragment and the APP intra-cellular domain (AICD) between aa711 and aa714. This pathway is called the non-amyloidogenic cleavage of APP, since the toxic A $\beta$ peptide is disrupted by the $\alpha$-secretase complex. Alternatively, APP can be cleaved in the late endosomal-lysosomal pathway by the $\beta$-secretase complex into sAPP $\beta$ and the membrane bound C99 fragment between aa671 and aa672. The C99 fragment is subsequently cleaved by the $\gamma$-secretase complex in the toxic $A \beta$ peptide and the AICD. Depending on the exact site of $\gamma$-secretase cleavage, $\mathrm{A} \beta 39-43$ is formed. $A \beta 40$, accounting for $90 \%$ of the $A \beta$ generation is less prone to self aggregation than the more toxic forms $A \beta 42$ and $A \beta 43$ [17]. $A \beta$ can aggregate into oligomers (dimers, trimers, etc.) and subsequently aggregate to form $A \beta$-fibrils. $A \beta$ fibrils finally aggregate together into the AD featuring $A \beta$-plaque deposits, one of the most important post mortem hallmarks of AD. However, despite high levels of amyloid deposition in $\mathrm{AD}$, the number of plaques often corresponds quite poorly with the degree of dementia [18]. Rather the amount of soluble oligomers play a determining role in the disease progression of $\mathrm{AD}$ [19]. $\mathrm{A} \beta$ oligomers might mediate neural system failure in different ways [3]: 1) defects in axonal transport which leads to dystrophic axonal processes [20], 2) loss of spines in the vicinity of $A \beta[21], 3)$ diminished neuronal responsiveness [22], and 4) disturbed memory related behavior upon infusion of oligomeric A $\beta$ [19]. Several mutations (e.g. the Swedish double mutation: K670N, $\mathrm{M} 671 \mathrm{~L})$ in the APP protein result in a shift from the non-amyloidogenic into the amyloidogenic pathway and therefore result in an increased production of $A \beta 40 / 42$, called early onset familial Alzheimer's, FAD. Also mutations compounds of the $\gamma-$ secretase complex (e.g. in PS1 or PS2) result in FAD. FAD mutations are often applied in the generation of in vivo and in vitro $\mathrm{AD}$ models. The studies performed on $\mathrm{AD}$ in this thesis will be bases on the $\mathrm{A} \beta$-hypothesis in different models of AD. 


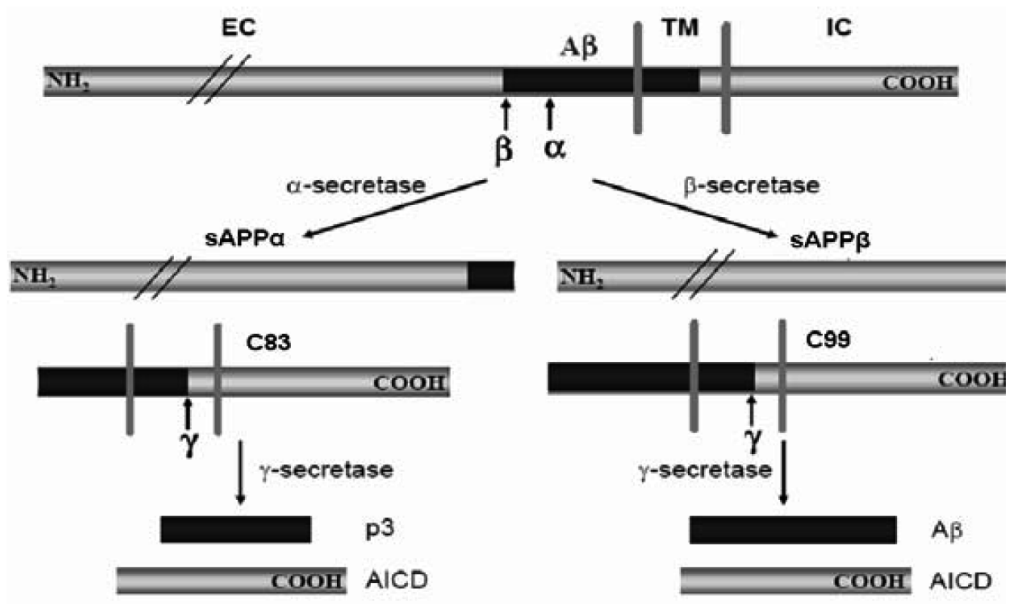

\section{Figure 1}

APP cleavage into the amyloidogenic and non-amyloidogenic pathway. EC extracellular, TM transmebrane, IC intracellular. Modified from Zheng and Koo Molecular Neurodegeneration 2006 [23]

\section{The Neurofibrillary Tangle hypothesis}

Neurofibrillary tangles (NFTs) are pathological protein aggregates within neuronal axons. NTFs are formed upon hyperphosphorylation of the microtubule associated-Tau protein. Tau protein normally binds to and stabilizes microtubules. However, hyperphosphorylated Tau aggregates into insoluble paired helical filaments (PHF), making microtubules inaccessible to motor proteins involved in anterograde transport which causes starvation of synapses. Thus, phosphorylated Tau containing neurons are unable to perform normal functions critical to the survival of a nerve cell. Changes in NFTs are among the earliest signs of AD [24]. After the death neuron has been removed, PHF remain as insoluble ghost tangles and constitute an important post mortem hallmark in AD. Tau protein is encoded in a single gene with six splice variants predominantly expressed in adult neurons [25]. A variety of mutations occur in the human Tau gene, i.e. causing Fronto Temporal Dementia and Parkinsonism linked to chromosome 17 (FTDP-17), a rare disorder characterized by intracellular neuronal and glial inclusions formed by PHF-like tau filaments, providing prove that Tau abnormalities can cause neurodegenerative diseased [26]. 
A link between phosphorylated Tau and $\mathrm{A} \beta$ is suggested; Tau phosphorylation increases in the vicinity of $\mathrm{A} \beta$ in vitro and in vivo. This hypothesis of $\mathrm{A} \beta$ inducing Tau hyperphosphorylation has been supported by the development of significant pathology and degeneration in cortical and subcortical brain regions in transgenic mice expressing both mutant human APP and Tau [27, 28]. However, other studies indicate that the relation between Tau and APP is more complex than initially thought $[3,29]$.

\section{The Cholinergic hypothesis}

It wasn't until the link between the memory loss in AD and specific cholinergic deficits in the brains of people suffering form $\mathrm{AD}$ became clear in mid seventies, that the cholinergic hypothesis emerged [30, 31]. Therefore, the cholinergic hypothesis was one of the earliest hypothesises on the etiology in AD. Lower levels of choline acetyltransferase (ChAT) were discovered in people with AD [32]. The links between cognitive impairment, cholinergic deficits, loss of central cholinergic neurons of the basal forebrain and altered processing of APP, eventually led to larger clinical studies that attempted to modulate the cholinergic system, increasing the signal strength [3]. These efforts culminated in the FDA approval of acetyl cholinesterase inhibitor for treatment of mild to moderate patients. However, the acetyl cholinesterase inhibitors are solely used for symptomatic treatment and do not stop or reverse the pathological disease progression. Therefore, the conclusion was drawn that acetyl choline deficiencies are probably not directly causally involved in $\mathrm{AD}$, but rather a result of widespread brain tissue damage.

\section{Other promising leads}

Besides the traditional etiology theories, several potential causal mechanisms have been suggested since the discovery of AD. Some of the most promising hypothesises are summarized in Jucker et al [3] and are briefly summarized below.

Firstly, an early hypothesis on development of dementia was the metabolic/energetic hypothesis. The hypothesis was based on the idea of reduced transfer of nutrients towards the brain, due to gradually increase in hardenings of the arteries. After being felt out of favor for several decades, the hypothesis, postulating an important role for cerebrovascular perfusion, oxidative metabolism, glucose hypometabolism or mitochondrial dysfunction play a crucial role in the etiology of dementia is now gaining support again. Two important lines of evidence exist. First, neuroimaging studies with positron emission tomography (PET) showed specific regional reduction 
in glucose utilization [33]. Second, epidemiological data show a strong relationship between vascular disease, hypertension and an increased risk for dementia [34].

Secondly, neuroinflammation, involved in the disease pathology of several neurodegenerative diseases, often involves excessive glial activation, apoptosis, overproduction of proinflammatory cytokines, oxidative stress-related enzymes, and various compounds of the complement cascade. Moreover, A $\beta$ deposition and NFTs in $\mathrm{AD}$ are associated with glial activation, neuronal loss and cytokine polymorphisms (e.g. IL1) $[35,36]$.

Thirdly, the most proximal events noticeable in the AD brain are the loss of synapses, dendrite pruning and cell death. Despite the ability of the healthy aging brain to from new synapses and to maintain dendrite arborization, the evolution of $\mathrm{AD}$ is featured by enormous neuronal and synapse loss, correlating well with the parallel cognitive decline [37-39]. However, despite several anatomical hallmarks detectable by Pittsburgh Compound (PiB) A $\beta$ PET scans, quantitative (MRI) scans, etc., the onset of pathologic changes in $\mathrm{AD}$ begins already years prior to the emergence of those overt clinical symptoms [40].

Fourthly, alterations in the brain cholesterol metabolism are strongly linked to the etiology, development and progression of AD. The brain cholesterol metabolism in $\mathrm{AD}$ will be the topic of this thesis; more in particular, the modulations in the brain cholesterol metabolism in the course of AD pathology will be described. The role of the cholesterol metabolism in $\mathrm{AD}$, known so far will be discussed in the following paragraphs. 


\section{The Brain Cholesterol Metabolism}

\section{Cholesterol Synthesis and Function}

5-Cholesten-3 $\beta$-ol or cholesterol is the principal sterol synthesized by animal cells. Other eukaryotes e.g. fungi and plants synthesize cholesterol as well, though in minimal amounts [41, 42]. In contrast, prokaryotes, like bacteria lack the ability of synthesizing cholesterol. The white crystalline powder was first discovered by François Poulletier de la Salle in 1769 in gallstones. Later, in 1815, the chemist Michel Eugène Chevreul rediscovered the molecule and called it cholesterol after the two Greek words chole- (bile) and stereos (solid), and added the chemical suffix -ol for the alcohol group on position 3 [43] (figure 2). Within "physiological" temperatures cholesterol is an important determinant to maintain fluidity of cell membranes and therefore warrants essential physiological functioning. The hydroxyl group on cholesterol interacts with the polar head groups of the membrane phospholipids and sphingolipids, while the bulky steroid and the hydrocarbon chain are embedded in the membrane, alongside the non-polar fatty acid chain of the other lipids [44]. In this structural role, cholesterol reduces the permeability of the plasma membrane to protons (positive hydrogen ions) and sodium ions [45]. Without cholesterol, cell membranes would need a wall like in bacteria and plants.

Cholesterol biosynthesis is a complex process involving more than 20 reaction steps and intermediates. All 27 carbon atoms of the cholesterol molecule (C27) are derived from acetyl-Coenzyme A (CoA). In a first step, acetyl-CoA and acetoacetyl-CoA are joined together to form 3-hydroxy-3-methylglutaryl-CoA (HMG-CoA). Next, the rate limiting, irreversible step in cholesterol synthesis is the reduction of HMG-CoA into mevalonate (C6) by HMG-CoA reductase (HMGCR), an integral membrane protein of endoplasmatic reticulum. Mevalonate is then converted into isopentyl pyrophosphate (C5) over three subsequent steps. Subsequently, three molecules of isopentenyl pyrophosphate condense to form farnesyl pyrophosphate $(\mathrm{C} 15)$ through the action of geranyl transferase. Two molecules of farnesyl pyrophosphate then merge to form squalene (C30) by the action of squalene synthase.

Squalene is first activated by squalene oxidase into squalene epoxide (2,3oxidosqualene) in a reaction that uses $\mathrm{O}_{2}$ and Nicotinamide Adenosine Dinucleotide Phosphate (NADPH). Squalene epoxide is then cyclized to form lanosterol (C30). Lanosterol is converted into cholesterol in a multistep process by the removal of three methyl groups, the reduction of one side-chain double bond, and the migration of the 
other double bond into the B-ring. This conversion either occurs via the Bloch pathway involving desmosterol or via the Kandutsch-Russel pathway involving lathosterol as most important intermediate (see figure 2 ) [46, 47]. The switch from the Bloch pathway towards the Kandutsch-Russel pathway is made by action of the 24 dehydroxycholesterol reductase (DHCR24) also know as the selective AD indicator gene (Seladin-1). Dhcr24 is an oxidoreductase which catalyzes the reduction of the delta-24 double bond of sterol intermediates of the Bloch pathway, e.g., lanosterol and desmosterol, hereby generating respectively 24,25-dihydro-lanosterol and cholesterol. Interestingly, there is evidence for differential use of the pathways, for instance during aging. In young mice, brain cholesterol is synthesized via the Bloch pathway, in old mice via the Kandutsch-Russel pathway [48]. In the brain cells, neurons contain predominantly precursors of the Kandutsch-Russel pathway including 7dehydrocholesterol and lathosterol, whereas astrocytes contain precursors of the Blochpathway, namely desmosterol, indicating different regulation mechanisms are active [49].

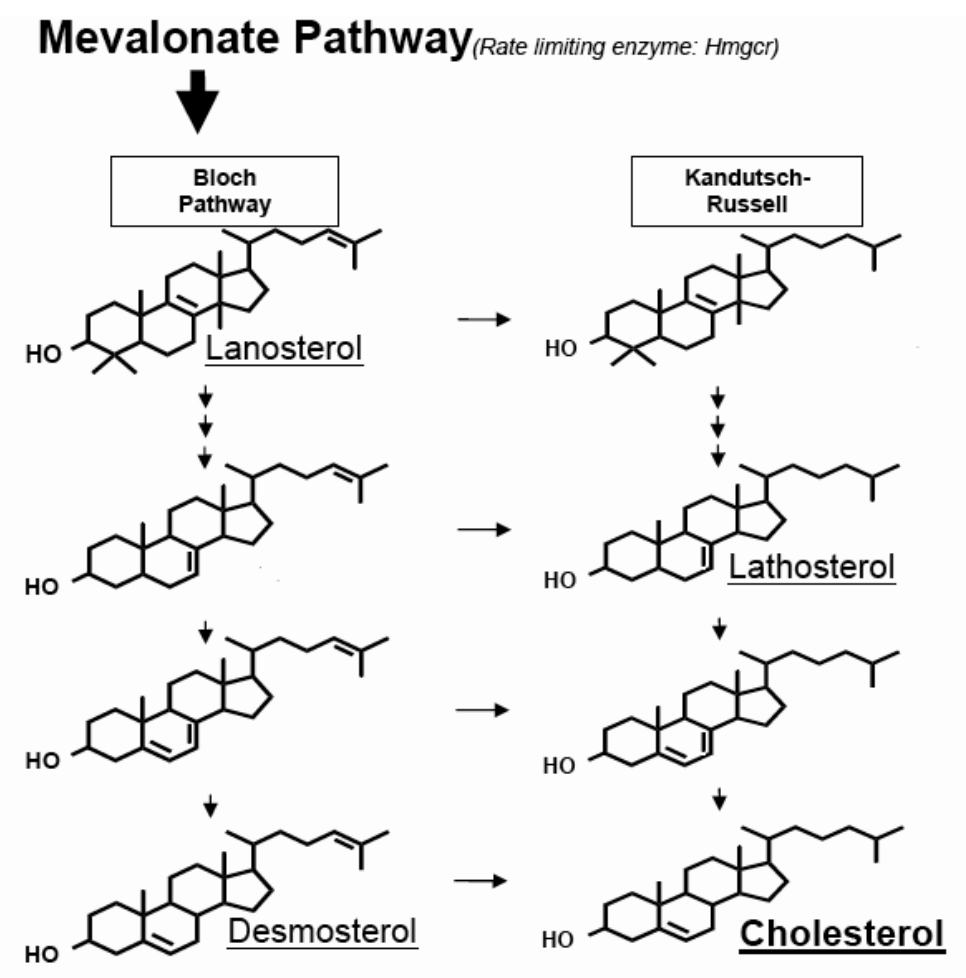

Figure 2

Biosynthesis of cholesterol trough the Bloch and the Kandutsch-Russell pathways. 
Cholesterol is involved in a broad range of physiological processes. Firstly, cell membranes require cholesterol to construct and maintain their flexible, though firm structure. The hydroxyl group on cholesterol interacts with the polar head groups of the membrane phospholipids and sphingolipids, while the bulky steroid and the hydrocarbon chain are embedded in the membrane, alongside the non-polar fatty acid chain of the other lipids. Cholesterol is an important constituent of membrane lipid rafts as will be discussed later.

Secondly, cholesterol is an integral and important constituent of myelin. Myelin sheets are compact monolayers of Schwann cells and oligodendrocytes formed around axons of neurons respectively in the peripheral and central nerve system (CNS). Insulation of axons by myelin sheets provides more efficient impulse conduction. Demyelinisation and consequently disturbance of signal transduction can lead to the severe neurological disorder, multiple sclerosis (MS) featured by focal accumulation of cholesteryl esters in the brain, generated during decomposition of myelin [50].

Thirdly, cholesterol aids in the absorption of fat soluble vitamins, e.g. vitamin A, vitamin $\mathrm{D}$, vitamin $\mathrm{E}$ and vitamin $\mathrm{K}$. In addition, 7-dehydrocholesterol, the last precursor of cholesterol in the Kandutsch-Russel pathway, is an important intermediate in the synthesis of Vitamin D in the skin. Also, adrenal gland hormones, e.g. cortisol, and aldosterone as well as the steroid sex hormones e.g. progesterone, estrogens, and testosterone are derived from metabolizing cholesterol. In the liver, cholesterol is converted into bile acids, which are stored in the gallbladder. Bile acids solubilize dietary fats and vitamins in the digestive tract and aid intestinal absorption of these molecules. Bile acids are the most important excretion mechanism of cholesterol and cholesterol derivates.

Cholesterol is abundantly present in the human body at rates of $\sim 2200 \mathrm{mg} / \mathrm{kg}$. The source of cholesterol in the human body is dual. Firstly, cholesterol is synthesized de novo from acetyl-CoA as discussed later $(\sim 10 \mathrm{mg} /$ day $/ \mathrm{kg})$. Secondly, cholesterol is dietary derivable at interindividual differing absorption rates (ranging from $30-70 \%$ accounting for $\sim 5 \mathrm{mg} / \mathrm{day} / \mathrm{kg})$. Similar amounts of cholesterol are excreted as fecal neutral sterol $(\sim 8 \mathrm{mg} / \mathrm{day} / \mathrm{kg})$ and acidic $(\sim 7 \mathrm{mg} / \mathrm{day} / \mathrm{kg})$ sterol excretion, bringing the daily cholesterol turnover rate at $\sim 15 \mathrm{mg} /$ day $/ \mathrm{kg}$ in humans [51]. In the circulation, cholesterol is transported as a constituent of lipoproteins together with triglycerides and phospholipids [52]. Depending on the density, the apolipoprotein present, and the fate of the lipoprotein particle, lipoproteins are divided grossly into: chylomicrons $(d<0.94$ $\left.\mathrm{g} \mathrm{cm}^{-3}\right)$, very low density lipoproteins (VLDL) $\left(\mathrm{d}<1.006 \mathrm{~g} \mathrm{~cm}^{-3}\right)$, intermediate density lipoproteins (IDL) $\left(1.006<d<1.019 \mathrm{~g} \mathrm{~cm}^{-3}\right)$, low density lipoproteins (LDL) $(1.019<$ $\left.d<1.063 \mathrm{~g} \mathrm{~cm}^{-3}\right)$, and high density lipoproteins (HDL) $\left(1.063<\mathrm{d}<1.21 \mathrm{~g} \mathrm{~cm}^{-3}\right)$. The density increases as the ratio triglycerides:cholesterol decreases [46]. 


\section{Brain Cholesterol Homeostasis}

The brain is the most cholesterol rich organ in the body. It contains about $25 \%$ of all free cholesterol in the body, while it represents only $2 \%$ of the total body weight. Essentially all cholesterol in the brain is unesterified. Radioactive tracer studies have demonstrated that cholesterol metabolism in the brain is almost entirely separated from cholesterol metabolism in the periphery ( $<1 \%$ flux) [53]. Whereas peripheral organs readily take up cholesterol from the blood in the form of LDL-particles and secrete it in the form of HDL particles, it is generally assumed that cholesterol can not cross the blood brain barrier (BBB) (figure 3) under non-diseased conditions [54]. The cerebral capillary endothelium is the anatomical substrate of the $\mathrm{BBB}$, isolating the brain neuropil from the systemic circulation. The cerebral endothelium lining the blood vessel lumen consists of a single layer of cells joined together by tight intracellular junctions. This layer of cells is supported by a basement membrane, which is the laminar structure formed by the fusion of the endothelial and glial vascular basement membrane. The end feet of the astrocytes make up a discontinuous sheath at the abluminal surface of the basement membrane, not allowing cholesterol transfer [52]. Therefore, the brain cholesterol metabolism is self regulating and most cholesterol present in the brain is assumed to be synthesized in situ [55].

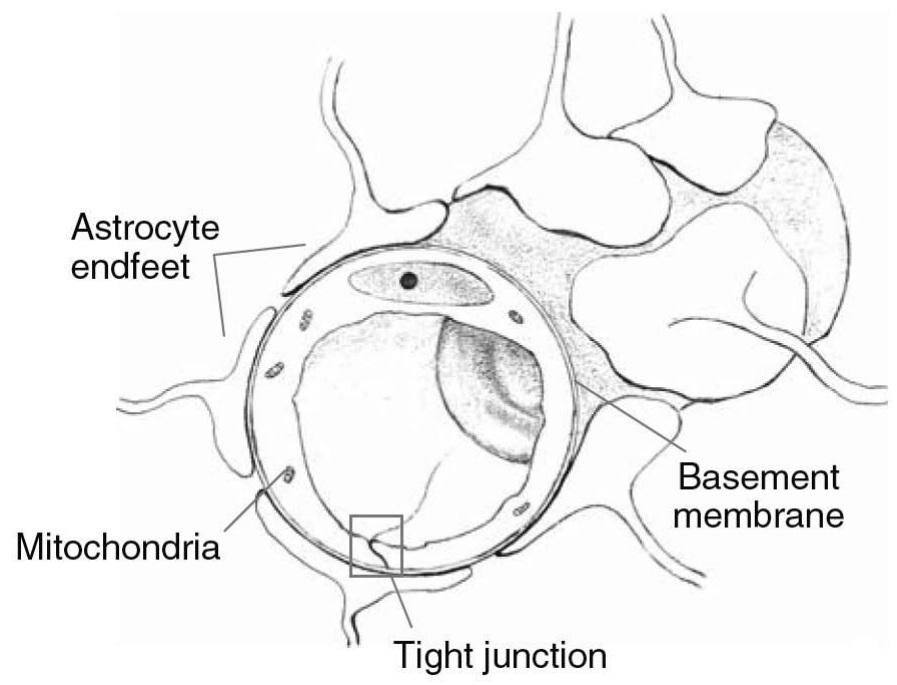

\section{Figure 3}

Schematic representation of the blood-brain barrier. The blood-brain barrier consists of endothelial cells lining the vessel wall, connected via tight junctions. These are surrounded by a thick basement membrane which is covered by astrocytic endfeet. [52] 
Despite the high cholesterol content, cholesterol turnover in the brain was found to be much slower, but far more stable than that in the rest of the body, e.g. 20-80 fold lower than in the liver [56]. However, cholesterol in the brain resides in three major compartments with different turnover rates. The larger pool $(70-80 \%$, or $\sim 260 \mathrm{mg} / \mathrm{kg}$ of the total $\sim 330 \mathrm{mg} / \mathrm{kg}$ ) has the slowest turnover and is present in the myelin membranes at half replacement times of 359 days $\sim 0.3 \%$ /day [57-59]. Of the remaining $\sim 70 \mathrm{mg} / \mathrm{kg}$, about $10 \%$ reside in neurons, which represent about $10 \%$ of the brain cells, and therefore $\sim 7 \mathrm{mg} / \mathrm{kg}$ of cholesterol. The remaining $\sim 63 \mathrm{mg} / \mathrm{kg}$ are present in glial cells [60-62]. The cholesterol turnover in the neuronal fraction is described to be very high i.e. pyramidal cells of the cortex together with the Purkinje cells of the cerebellum are calculated to have a cholesterol turnover of more than $20 \%$ /day, whereas whole body cholesterol turnover is $0.8 \%$ /day. A continuous turnover of cholesterol in neurons may facilitate the cells' ability for efficient and quick adaptation of cholesterol homeostasis required for dynamic structural changes of neurons, their extensions, and their synapses during synaptic plasticity [63]. Neuronal cholesterol content is considered to be a crucial parameter in appropriate neuronal functioning [52].

The metabolism of cholesterol depends on three main aspects: synthesis, transport and catabolism. The major site of cholesterol synthesis in the brain is located in the astrocytes. In early developmental stages, neurons can synthesize cholesterol themselves. In contrast, mature neurons mainly shut down the cholesterol synthesis machinery and rely on astrocytes for this energy consuming process [64, 65]. Evidence is available that astrocytes secrete cholesterol via the ATP binding cassette transporter A1 (ABCA1), ABCG1 and ABCG4 towards poorly lipidated discoidal ApoE generating HDL-like particles in order to supply neurons with cholesterol [66-69]. As such, cholesterol is required for the formation of new synapses, axonal outgrow, and vesicle exocytose, which are all essential for learning and memory processes [70-72]. Peripheral cholesterol is not able to cross the BBB, the other way around is true too; cholesterol can not leave the brain as such. Therefore, cholesterol is metabolized into the brain specific oxysterol 24(S)-OHcholesterol, also called cerebrosterol formed by the neuronal enzyme cholesterol 24(S)- hydroxylase (CYP46A1) [73, 74]. 24(S)OHcholesterol is, in contrast with cholesterol able to traverse the BBB [75, 76]. At first glance, it looks quite controversially that a compound, more polar than cholesterol is able to pass a lipophilic barrier. As with other plasma membranes, the membranes of the endothelial cells are permeable to water [77]. Introduction of an hydroxyl group in the side-chain of the cholesterol molecule leads to a local reordering of membrane phospholipids such that it is energetically more favorable to expel the oxysterol [78]. When a sterol like cholesterol is hydroxylated, there is an increase in the maximal aqueous activity, but a reduction in the passive permeability coefficient. However, the 
increase in solubility is proportionately greater than the reduction in the permeability coefficient, so that the net effect of hydroxylation is to greatly increase the maximal rate of passive diffusion of the molecule across the BBB. 24(S)-OHcholesterol accounts for $64 \%$ of cholesterol efflux to the periphery [62, 75]. The remainder is secreted via an unknown pathway that may involve ABCA1 and ApoE [79, 80].

\section{Regulation of the Brain Cholesterol Metabolism.}

If neurons for their cholesterol supply predominantly rely on astrocytes, it can be questioned how neurons communicate with these cells in order to request sufficient amounts of cholesterol for optimal functioning. An important communication route is via 24(S)-OHcholesterol produced by neurons. 24(S)-OHcholesterol acts as a signal molecule that activates the nuclear transcription factor Liver X Receptor (LXR) in astrocytes and by that induces cholesterol turnover in astrocytes [81]. The selective activation of LXR in astrocytes and not in neurons is explained by the 10- to 20-fold higher basal LXR $\alpha$ and $-\beta$ expression level in astrocytes [81]. In astrocytes cholesterol synthesis is thought to be regulated by two master transcription regulation systems, i.e., sterol regulatory element binding proteins (SREBPs) and LXRs. SREBP activation increases the transcription of genes involved in increasing cellular cholesterol levels [82], while LXR activation facilitates the disposition of cholesterol from cells towards ApoE particles [83]. It is assumed that neurons depend on astrocytal ApoE secretion for their cholesterol supply [64].

LXRs, as part of the nuclear receptor family, consist of two isoforms, namely: LXR $\alpha$ (NR1H3) and LXR $\beta$ (NR1H2). Transcription of LXR responsive genes is initiated upon ligand binding to the ligand-binding-domain and subsequent heterodimerisation with retinoid $\mathrm{X}$ receptor $(\mathrm{RXR})$. The LXR-RXR heterodimer translocates to the nucleus where the DNA-binding-domain from the LXR binds to LXR responsive elements (LXRE) in the promotor region of LXR responsive genes. The LXR responsive genes are mostly genes involved in cholesterol efflux, including ABCA1, ABCG1, APOE, APOCI and genes involved in fatty acid synthesis, like SREBP1c and Fatty Acid Synthase (FASN). LXR $\alpha$ is mainly expressed in liver, adrenal glands, intestine, adipose tissue and macrophages, whereas LXR $\beta$ is expressed ubiquitously [84]. LXR $\alpha$ and LXR $\beta$ are both present in the brain and thought to be involved in the regulation of brain cholesterol homeostasis [85].

Several endogenous ligands for LXR are described: cholesterol precursors (Follicular fluid meiosis-activating sterol (FF-MAS), Testis-MAS (T-MAS) and desmosterol) and cholesterol metabolites (oxycholesterols) [86]. Oxycholesterols are the best known endogenous agonists of LXRs. Besides the endogenous sterol based LXR agonists, also 
sterols of plant or fungal origin are suggested to be LXR agonists respectively phytosterols and ergosterol. Synthetic compounds including T0901317 and GW3965 are described to be LXR agonist as well [86].

SREBP, residing in the ER, is activated in sterol-poor conditions and transported to the Golgi complex where it undergoes proteolytic processing. Subsequently, this fragment is imported into the nucleus, where it switches on the transcription of HMGCR and other sterol-regulated genes. Binding of sterols to the two chaperones (SCAP) and INSIG1 induces conformational changes resulting in the inhibition of the transport of SREBP from the ER to the Golgi. Binding of cholesterol to SCAP and binding of oxysterols, such as 24(S)-OHcholesterol in the brain, to INSIG causes these chaperones to bind one another thereby preventing SCAP from escorting SREBP to the Golgi by making it inaccessible to COPII and preventing the increase in transcription of gene products [87]. Alternatively, cholesterol homeostasis can be regulated at the level of the proteasomal degradation of HMGCR [88].

Upon increased 24(S)-OHcholesterol production, neurons specifically increase ABCG4 expression [81]. Recently, in vivo evidence from transgenic mouse experiments has been provided for a role for ABCG4 in sterol efflux in the brain and that ABCG1 and ABCG4 have overlapping functions in astrocytes, promoting efflux of cholesterol, desmosterol and possibly other sterol biosynthetic intermediates to HDL [67]. The selective up-regulation of ABCG4 in neuroblastoma cells by 24(S)-OHcholesterol suggests a possible role for this transporter in oxysterol transport [81].

Several pathways are suggested to be involved in neuronal cholesterol internalization. Receptors that have been suggested to be involved are: LDLR, LRP1, LR11, apoER2 and VLDLR [89, 90]. Low density lipoprotein receptor related protein 1 (LRP1) is detected predominantly in neurons and in activated astrocytes [91-93]. A number of in vitro and in vivo studies indicate a role for the LRP in the internalization of cholesterol by neurons [94-97]. Besides LRP neurons express LDLR mainly at the soma, apoER2 mostly in distal axons, LR11 in cell bodies/proximal axons, and also SCARBI [98, 99].

\section{Plant Sterols in the Brain}

Another group of dietary available, bio-active sterols are plant sterols and their saturated forms, plant stanols (collectively referred to as phytosterols). Phytosterols are sterols with a high structural and functional similarity to cholesterol. However, none of the mammalian cells are able to synthesize plant sterols from any precursor [100]. The sole source of phytosterols is dietary intake. Phytosterols form about $20 \%$ of the total sterols consumed in the western type diet [101]. Although depending on the nature of the diet, most commonly available phytosterols are sitosterol (65\%), campesterol 
(32\%), and stigmasterol (3\%). Furthermore, traces of other phytosterols e.g. brassicasterol, avenasterol and fucosterol as well as the saturated forms of the most predominantly available phytosterols (sitostanol, campestanol and stigmastanol) are retrieved in the diet. The main phytosterols differ chemically only from cholesterol by an additional ethyl or a methyl group at C24 and/or a double bound at C22 [102, 103].

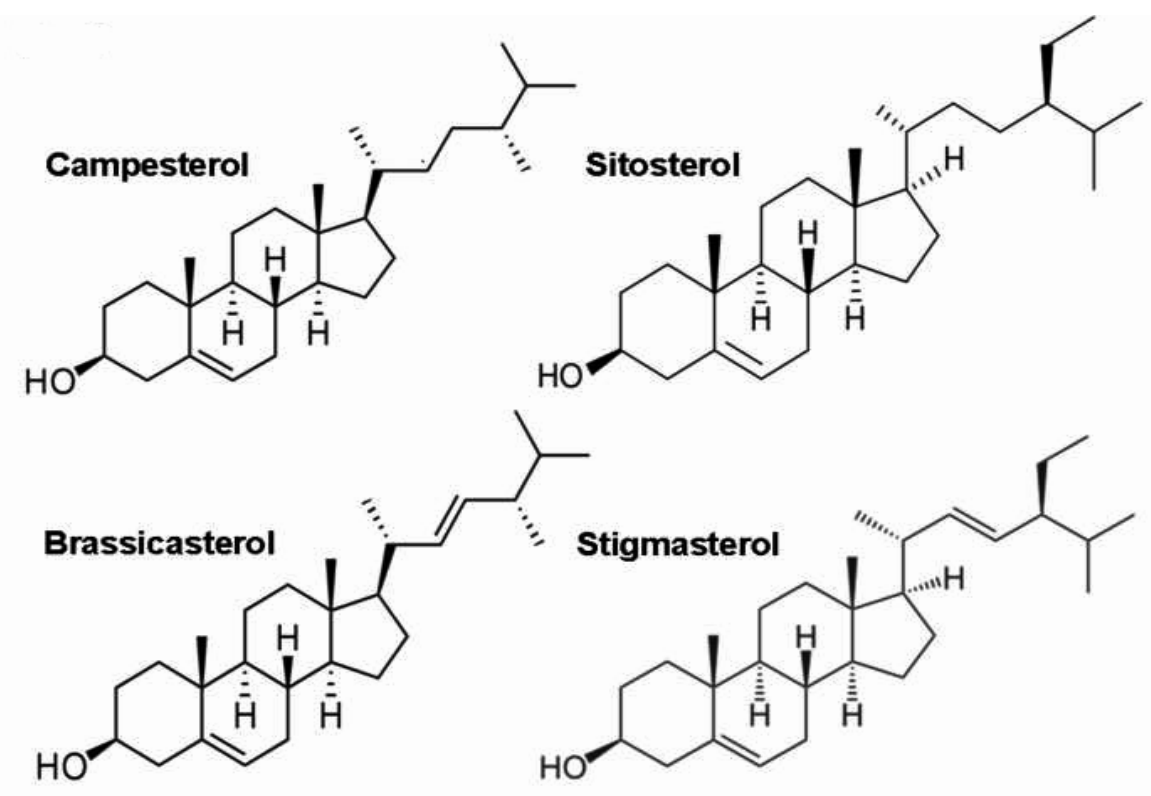

Figure 4

Plant sterols differ chemically only from cholesterol by an aditional methyl (campesterol) or ethyl goup (sitosterol) at C24 and/or a double bound at C22 (stigmasterol and brassicasterol).

In contrast with the about $30-70 \%$ absorption of the dietary available cholesterol, only less than $5 \%$ of total dietary available phytosterols are absorbed from the intestine, with an even lower absorption rate for plant stanols $(0.02-0.3 \%)$ [100, 104-106]. Both phytosterols and cholesterol are taken up by the Nieman-Pick C1-like-1 (NPC1L1) transporter in the brush border of the enterocytes [107, 108]. In comparison to dietary cholesterol, phytosterols are hardly esterified because they are a poor substrate for acetyl-CoA acetyltransferase 2 (ACAT2) [109]. Therefore, it is generally assumed that only a fraction of the available phytosterols is esterified and subsequently basolaterally secreted together with cholesterol esters into chylomicrons towards the lymphatic system or alternatively via the ABCA1 transporter into ApoAI containing HDL [110, 
111]. Accumulation of free phytosterols in enterocytes is reduced by apical secretion of the unesterified phytosterols into the intestinal lumen by the ABCG5/ABCG8 heterodimer [112]. After phytosterol uptake by the hepatocytes, the remaining phytosterols are mainly resecreted into the bile and only partially released into the serum after esterification by lecithin-cholesterol acyltransferase (LCAT) [113]. Despite elaborated secretion mechanisms, only a small but significant fraction of plant sterols ends up as components of VLDLs and can be retrieved in the serum [114]. Consequently, it was shown that plant sterols can accumulate in the several peripheral tissues including the brain [115-117]. Since serum cholesterol is not able to cross the $\mathrm{BBB}$, the exact mechanisms by which the structurally similar plant sterols can accumulate in the brain remains to be elucidated. The brain cholesterol metabolism is a well orchestrated complex system, necessary in optimal brain functioning. Disturbances herein are often related to severe disorders featured by cognitive disabilities such as in Smith-Lemli-Opitz, Tangier Disease, Niemann-Pick disease type $\mathrm{C}$ and also $\mathrm{AD}$ [118-120]. The role of plant sterols in pathogenesis of $\mathrm{AD}$ will be discussed in this thesis.

Defects in the ABCG5 or ABCG8 transporter that form a functional heterodimer, result in accumulation of plant sterols, giving rise to the human inborn error of metabolism "sitosterolemia". These patients develop severe atherosclerosis at very young age [121]. It is thought that ABCG5/ABCG8 heterodimers function by preventing plant sterols from being retained by the body by limiting intestinal absorption and facilitating secretion into bile. Apart from plant sterols, cholesterol is also transported by the ABCG5/ABCG8 heterodimer.

Abcg5-null mice have been generated recently and have been characterized with respect to sterol metabolism [122]. These mice display strongly elevated plasma levels of the plant sterols sitosterol and campesterol. We found that Abcg5-knockout mice accumulate plant sterols in their brains [117]. Levels of campesterol were increased 7fold and levels of sitosterol 12 -fold $(\mathrm{p}<0.0001)$. It can be hypothesized that plant sterol levels in the brain increase even further when the mice are fed a diet rich in plant sterols [117]. The influence of plant sterols on brain functioning and the brain cholesterol metabolism will be discussed in this thesis.

\section{Lipid Rafts and Membrane Domains}

Biological animal membranes consist of a lipid composition with integrated functional membrane proteins. Although the bulk of the membrane bilayer has been considered a homogeneous liquid-disordered $\left(l_{d}\right)$ phase, it is generally assumed that transient lateral heterogeneities coexist in a liquid-ordered $\left(1_{0}\right)$ phase, so called lipid rafts [123]. Lipid 
raft domains are enriched in free cholesterol and saturated lipids including sphingomyelin, glycosphingolipids, cerebrosides and gangliosides, whereas the main membrane phosphopholipids (phosphatidylcholine and phosphatidylethanolamine) have a reduced presence [124]. Cholesterol plays an important role in reducing lipid disorder, mainly present at the cytofacial leaflet of the membrane [125]. Depending on the detergent in which they are isolated, their composition can vary. Therefore, lipid rafts are often referred to as detergent resistant membranes (DRMs). Cholesterol is able to promote the separation between $l_{d}$ and $l_{\mathrm{o}}[126,127]$.

Lipid rafts are lipid ordered integral membrane scaffolds, acting as a movable platform for processes involved in e.g. membrane trafficking, signal transduction, and regulation of the activity of membrane proteins [124, 128-130]. Proteins are assumed to be specifically included or excluded from the membrane rafts, thereby providing a mechanism for confined protein clustering [87]. In cells, cholesterol concentrations influence the domain partitioning and biological activity of proteins that co-isolate in DRMs [131, 132]. Many studies have shown that depletion of cholesterol from cells leads to the disruption of lipid rafts and the release of raft constituents into the bulk plasma membrane [124]. Since cholesterol is crucial for the generation of the $1_{\mathrm{o}}$ phase, alterations in the cholesterol content of cells should lead to changes in the scaffold properties of these domains [124]. Moreover, membrane cholesterol is essential for the structure and function of invaginated caveola and clathrin-coated pits, including caveola-dependent and clathrin-dependent endocytosis [133]. The role of cholesterol in such endocytosis can be investigated by using methyl beta cyclodextrin $(\mathrm{M} \beta \mathrm{CD})$ to remove cholesterol from the plasma membrane [134]. The presence of plant sterols in the cells is expected to influence the fluidity of cellular membranes. 


\section{AD \& Cholesterol metabolism}

\section{Plasma Cholesterol and AD}

FAD is caused by mutations in genes involved in the APP metabolism, namely APP, PS1 and PS2. The cause is less clear in the most occurring, sporadic form of AD. Yet, several epidemiological studies have stressed an association between high plasma cholesterol, mainly in mid age, and increased susceptibility to sporadic late-onset AD [135-137]. In parallel, diet induced hypercholesterolemia enhanced accumulation of A $\beta$ in the brains of transgenic $\mathrm{AD}$ mice and rabbits [138, 139]. In contrast, another group demonstrated decreased plasma cholesterol levels during aging in a transgenic mouse model of AD [140]. Therefore, the role of circulating cholesterol on the risk on $\mathrm{AD}$ development is not clear.

Circulating cholesterol is assumed not to cross the BBB; the potential effect of plasma cholesterol on the development of $\mathrm{AD}$ is expected to be rather secondary. Firstly, high plasma cholesterol levels are strongly correlated with increased development of atherosclerosis. Increased brain vessel atherosclerosis together with a subsequent reduction of blood and oxygen supply to the CNS resulting in cerebral parenchyma stress, is associated with AD [141]. Secondly, despite cholesterol not being able to cross the $\mathrm{BBB}$, more polar cholesterol metabolites, e.g. 27-OHcholesterol can cross the BBB. 27-OHcholesterol is suggested to be a potential link between peripheral and central cholesterol metabolism in AD [142]. A direct effect of circulating cholesterol on $\mathrm{AD}$ development risk is rather unlikely.

\section{Brain Cholesterol Transport in AD}

Perturbations in the brain cholesterol transport are strongly linked to AD. Firstly, the presence of the $\varepsilon 4$ allele of APOE (APOE4) is the main genetic risk factor for sporadic, late onset $\mathrm{AD}$ [14]. In the human population, APOE consists of 3 isoforms, each containing 299 amino acid residues: APOE2, APOE3 and APOE4. APOE2 differs from E3 by a single cysteine substituted for an arginine at position 158. E4 differs from E3 by an arginine substitution for a cysteine residue at position 112 [143]. ApoE protein functions as lipid transporter in lipoproteins in periphery and is the most important apolipoprotein involved in cerebral cholesterol transport. In contrast to humans, mice only have 1 allele for ApoE. The exact molecular mechanism underlying the relationship between the APOE4 allele and the late onset sporadic $\mathrm{AD}$, remains to 
be determined. However, recent relationships have been suggested. ApoE4 promotes $A \beta$ production, aggregation and amyloid plaque formation and reduces the clearance of $\mathrm{A} \beta$ by a weaker affinity for $A \beta$ peptides than for the other ApoE variants [144-146]. Consequently, the binding of ApoE4-A $\beta$ to LRP-1 would result in a compromised LRP-1 mediated A $\beta$ endocytosis and degradation [96, 147].

Secondly, alterations in expression, functionality, and metabolism of lipoprotein receptors are associated to AD. The amyloidogenic cleavage of APP, partially occurs in the endocytic pathway [148]. APP-interacting proteins and lipids involved in endocytosis modulate the trafficking and processing of APP into the endocytic compartments. Cholesterol and LDLR family members (a.o.: LDLR, LRP-1, LRP-1B, ApoER2, LR11, VLDLR) play a pivotal role in cellular localization and trafficking of APP [149]. Expression levels, cofactors, and mutations in either of these compounds imply consequences in APP processing.

Thirdly, cholesterol transport from astrocytes towards neurons is required for optimal neuronal functioning. As discussed above, LXRs are master regulators of cholesterol transport [64]. A decreased expression of LXR-target genes involved in cholesterol transport and synthesis (ABCA1, APOE, and DHCR24) is associated with AD development [150-153]. Improved memory performances and decreased AD-pathology are described upon LXR activation [144, 154]. Several LXR dependent mechanisms, including increased $A \beta$ degradation, decreased $A \beta$ production, and reduced inflammatory response in $\mathrm{AD}$ are described $[144,155,156]$. LXR activation in relation to cholesterol turnover and memory performances in $\mathrm{AD}$ will be discussed as part of this thesis.

Fourthly, several studies have shown that statins, cholesterol synthesis inhibitors, lower the risk on late-onset sporadic $\mathrm{AD}[157,158]$. Since other groups did not find this association [159], doubts exist concerning the role of statins in neurodegeneration [160]. Moreover, deficits in the brain cholesterol synthesis and transport are often associated with neurodegenerative diseases [153, 161]. Therefore, potential beneficial effects of statins are more likely expected to be outside the reduced cholesterol synthesis, e.g. decreased $\gamma$-secretase activity [157], anti-inflammatory activity [162], and reduction in geranylgeranyl isoprenoids levels [163].

Age depended alterations in the brain cholesterol metabolism in the APPSLxPS1mut mouse model, will be subject of this thesis.

\section{Neuronal Membrane Cholesterol and AD}

Different hypothesises on the role of membrane cholesterol in AD are postulated. Discrepancies in the literature are summarized into two models by Ledesma and Dotti 
[125]. The first model postulates that high membrane cholesterol is in favor of developing AD. In line with this hypothesis, Simons and coworkers demonstrated that reduction of plasma membrane cholesterol in APP overexpressing cultured hippocampal rat neurons by statins and cyclodextrin treatment resulted in drastic reduction of $\mathrm{A} \beta$ production [164]. It is assumed that the enzymes involved in the "beneficial" non-amyloidogenic cleavage of APP, $\alpha$-secretase is mainly localized in the non-lipid rafts containing membrane compartment, whereas $\beta$-secretase and $\gamma$-secretase are mainly localized within lipid rafts [165]. The model assumes two pools of APP exist: the larger non-lipid raft associated and the smaller lipid raft associated APP pool. Even if the majority of APP is in non-raft domains, allowing efficient processing by $\beta$ secretase (both found in raft and non-raft domains), the resulting $\beta$-C-terminal fragments ( $\beta$-CTFs) concentrate in lipid raft domains, allowing efficient processing by $\gamma$-secretase, which is associated with lipid rafts $[149,166,167]$. Decrease of membrane cholesterol content is believed to disrupt lipid raft associated $\beta$ - and $\gamma$-secretase and therefore reduced $A \beta$ generation [149]. High concentrations of cholesterol would promote clustering of APP and $\beta$-CTFs with $\beta$ - and $\gamma$-secretase containing lipid rafts, increasing $A \beta$ generation [168]. In addition, ApoE4 is suggested to improve clustering of APP and BACE in the cholesterol rich lipid raft [160]. Therefore, decreasing membrane cholesterol content in APP overexpressing cells reduces the production of $\mathrm{A} \beta$. However, it is shown that $\mathrm{AD}$ patients, in contrast to the previous hypothesis, have a moderate decreased hippocampal membrane cholesterol content [169].

The second model suggests that membrane cholesterol is important in the prevention of $\mathrm{A} \beta$ generation. In parallel to this hypothesis, it was shown that $\mathrm{AD}$ patients have lower neuronal membrane cholesterol content than controls [169]. Abad-Rodriguez and colleagues show that decreased membrane cholesterol content results in a delocalization of $\beta$-secretase from the lipid raft compartment towards the non lipid raft. As consequence, an increased interaction from $\beta$-secretase with the large non lipid raft associated APP pool, results in increased levels of A $\beta$ generation [170]. Moreover, reduced membrane cholesterol content results in a decreased activity of plamin, a membrane associated A $\beta$ degrading enzyme [169]. Also in favor of this model is the down regulation of DHCR24, an enzyme important in cholesterol synthesis as described above, in affected areas of AD brains [153]. Inhibition of ACAT1 reduces the intracellular cholesterol esterification and storage, leaving more free cholesterol available for the membrane compartment, results in decreased $A \beta$ production [171]. ApoE is necessary to transport $A \beta$ in microglial insulin degrading enzyme (IDE) mediated clearance of $A \beta$ [144]. In addition, ApoE4-derived glial cells have reduced ability to release cholesterol to neuronal receptors [68]. Neurons depend on cholesterol for synapse formation and axonal outgrowth [70-72]. ApoE4 positive AD patients 
show a moderate decrease in hippocampal membrane cholesterol [169]. Together this is in contrast with the high cholesterol hypothesis and supports the rather beneficial effect of cholesterol on AD.

Moreover, most experiments supporting the first hypothesis were performed in APP overexpressing cells in contrast to the more physiological models supporting the second model $[125,160]$. In addition, cholesterol in the neurons is involved in learning and memory processes $[63,172]$. The brain cholesterol metabolism is strictly regulated and severe disturbances of this homeostasis in both directions might be involved in $\mathrm{AD}$ development. Evidence indicates that cholesterol intracellular trafficking and distribution are relevant in the APP processing pathways. However, it remains undetermined whether alterations in the brain cholesterol metabolism are cause or consequence in the $\mathrm{AD}$ pathology.

\section{Plant Sterols and AD}

Recently, it was shown that plant sterols accumulate in the brain [115-117]. The exact fate of plant sterols in the brain remains to be determined. Incorporation of plant sterols in animal membranes can modulate the membrane composition and consequently membrane organization depending cellular functioning [77]. Accumulation of plant sterols in the brain may interfere with brain cholesterol homeostasis. Besides a cell specific up-regulation of APOE expression in astrocytes [117], no effects of plant sterols on the brain cholesterol metabolism are shown so far. Due to the structural parallel to cholesterol, interference with cholesterol depending functions, including the role in $\mathrm{AD}$ development can be expected. Therefore, the role of plant sterols on $\mathrm{AD}$ pathology will be addressed in this thesis. 


\section{The aim of the thesis}

The aim of the thesis was to elucidate whether specific modulation of the brain cholesterol metabolism could influence AD pathology.

In chapter II of the thesis, the brain cholesterol metabolism of an AD mouse model, assumed to posses a more physiological expression pattern of the involved APP gene, namely the APPSLxPS1mut mouse was examined. Brains and serum of APPSLXPS1mut mice and control littermates were examined at different ages: at the pre-plaque stage (9 months) versus end stage pathology (21 months). Different parameters in the brain cholesterol metabolism were measured and compared: cholesterol precursors, cholesterol, cholesterol metabolites and genes involved in cholesterol synthesis, transport and metabolizing. The aim was to see whether alterations are preceding or following AD related pathology.

In chapter III, APPSLxPS1mut mice and littermates were subjected to an LXR agonist (T0901317) versus control treatment at an end stage AD pathology level. Evolution of cognitive performances, amyloid deposition, and cholesterol turnover were topic of the research.

In chapter IV, dietary available plant sterols, which are structurally very similar to cholesterol, were investigated for their transfer across the blood-brain barrier (BBB) both in in vitro and in vivo models. Subsequently we investigated where plant sterols will accumulate beyond the BBB and whether plant sterols can modulate the processing of the APP protein in a neuronal cell model, stably overexpressing APP.

In chapter $\mathbf{V}$, the consequences of an increased presence of the plant sterols in the brain on normal brain functioning were examined. A phytosterolemic mouse model, Abcg5-/- mice, was compared with Abcg5+/+ littermates for memory and learning on the one hand and mood and anxiety related behavior on the other hand.

In chapter VI, we hypothesize, based on the solely exogenous nature of plant sterols in the CSF and the impaired choroid plexus function in $\mathrm{AD}$, that plant sterol concentrations in CSF are altered in AD pathology and therefore can be added to the relevant spectrum of biomarkers for $\mathrm{AD}$. CSF and plasma plant sterol concentrations will be compared between $\mathrm{AD}$ patients and age and gender matched non-demented "healthy" controls, applying age, gender, CSF phospho-tau, CSF A $\beta_{42}$, CSF:plasma albumin quotient, presence of the APOE4 allele and plasma creatinine as potential covariates. 


\section{References}

1. Zheng, H. and E.H. Koo, The amyloid precursor protein: beyond amyloid. Mol Neurodegener, 2006. 1: p. 5 .

2. Clegg, A., et al., Clinical and cost-effectiveness of donepezil, rivastigmine, and galantamine for Alzheimer's disease. A systematic review. Int J Technol Assess Health Care, 2002. 18(3): p. 497-507.

3. Brookmeyer, R., et al., Forecasting the global burden of Alzheimer's disease. Alzheimers Dement, 2007. 3(3): p. 186-91.

4. Jucker, M., et al., Alzheimer: 100 Years and Beyond, ed. Springer-Verlag. 2006, Berlin Heidelberg

5. Tomlinson, B.E., G. Blessed, and M. Roth, Observations on the brains of non-demented old people. J Neurol Sci, 1968. 7(2): p. 331-56.

6. Tomlinson, B.E., G. Blessed, and M. Roth, Observations on the brains of demented old people. J Neurol Sci, 1970. 11(3): p. 205-42.

7. Katzman, R., Editorial: The prevalence and malignancy of Alzheimer disease. A major killer. Arch Neurol, 1976. 33(4): p. 217-8.

8. $\quad$ Breteler, M.M., et al., Epidemiology of Alzheimer's disease. Epidemiol Rev, 1992. 14: p. 59-82.

9. Hooijmans, C.R. and A.J. Kiliaan, Fatty acids, lipid metabolism and Alzheimer pathology. Eur J Pharmacol, 2008. 585(1): p. 176-96.

10. Wang, X.P. and H.L. Ding, Alzheimer's disease: epidemiology, genetics, and beyond. Neurosci Bull, 2008. 24(2): p. 105-9.

11. Rademakers, R., M. Cruts, and C. Van Broeckhoven, Genetics of early-onset Alzheimer dementia. ScientificWorldJournal, 2003. 3: p. 497-519.

12. Tanzi, R.E. and L. Bertram, New frontiers in Alzheimer's disease genetics. Neuron, 2001. 32(2): p. 1814.

13. Van Duijn, C.M., et al., Interaction between genetic and environmental risk factors for Alzheimer's disease: a reanalysis of case-control studies. EURODEM Risk Factors Research Group. Genet Epidemiol, 1994. 11(6): p. 539-51.

14. Zekanowski, C., et al., Genetic aspects of Alzheimer's disease. Acta Neurobiol Exp (Wars), 2004. 64(1): p. 19-31.

15. Travis, J., New piece in Alzheimer's puzzle. Science, 1993. 261(5123): p. 828-9.

16. Selkoe, D.J., Toward a comprehensive theory for Alzheimer's disease. Hypothesis: Alzheimer's disease is caused by the cerebral accumulation and cytotoxicity of amyloid beta-protein. Ann N Y Acad Sci, 2000. 924: p. 17-25.

17. Neve, R.L., E.A. Finch, and L.R. Dawes, Expression of the Alzheimer amyloid precursor gene transcripts in the human brain. Neuron, 1988. 1(8): p. 669-77.

18. Trzesniewska, K., M. Brzyska, and D. Elbaum, Neurodegenerative aspects of protein aggregation. Acta Neurobiol Exp (Wars), 2004. 64(1): p. 41-52.

19. Arriagada, P.V., et al., Neurofibrillary tangles but not senile plaques parallel duration and severity of Alzheimer's disease. Neurology, 1992. 42(3 Pt 1): p. 631-9.

20. Walsh, D.M., et al., Naturally secreted oligomers of amyloid beta protein potently inhibit hippocampal long-term potentiation in vivo. Nature, 2002. 416(6880): p. 535-9.

21. Stokin, G.B., et al., Axonopathy and transport deficits early in the pathogenesis of Alzheimer's disease. Science, 2005. 307(5713): p. 1282-8.

22. Spires, T.L., et al., Dendritic spine abnormalities in amyloid precursor protein transgenic mice demonstrated by gene transfer and intravital multiphoton microscopy. J Neurosci, 2005. 25(31): p. 7278-87.

23. Kamenetz, F., et al., APP processing and synaptic function. Neuron, 2003. 37(6): p. 925-37.

24. Braak, H., et al., Vulnerability of select neuronal types to Alzheimer's disease. Ann N Y Acad Sci, 2000. 924: p. 53-61.

25. Lee, V.M., M. Goedert, and J.Q. Trojanowski, Neurodegenerative tauopathies. Annu Rev Neurosci, 2001. 24: p. 1121-59. 
26. Trojanowski, J.Q. and V.M. Lee, The role of tau in Alzheimer's disease. Med Clin North Am, 2002. 86(3): p. 615-27.

27. Goedert, M., et al., Cloning and sequencing of the cDNA encoding a core protein of the paired helical filament of Alzheimer disease: identification as the microtubule-associated protein tau. Proc Natl Acad Sci U S A, 1988. 85(11): p. 4051-5.

28. Lewis, J., et al., Enhanced neurofibrillary degeneration in transgenic mice expressing mutant tau and $A P P$. Science, 2001. 293(5534): p. 1487-91.

29. Dermaut, B., et al., A novel presenilin 1 mutation associated with Pick's disease but not beta-amyloid plaques. Ann Neurol, 2004. 55(5): p. 617-26.

30. Perry, E.K., et al., A cholinergic connection between normal aging and senile dementia in the human hippocampus. Neurosci Lett, 1977. 6(1): p. 85-9.

31. Davies, P. and A.J. Maloney, Selective loss of central cholinergic neurons in Alzheimer's disease. Lancet, 1976. 2(8000): p. 1403.

32. Whitehouse, P.J., et al., Alzheimer's disease and senile dementia: loss of neurons in the basal forebrain. Science, 1982. 215(4537): p. 1237-9.

33. Blass, J.P. and G.E. Gibson, The role of oxidative abnormalities in the pathophysiology of Alzheimer's disease. Rev Neurol (Paris), 1991. 147(6-7): p. 513-25.

34. de la Torre, J.C., How do heart disease and stroke become risk factors for Alzheimer's disease? Neurol Res, 2006. 28(6): p. 637-44.

35. Combs, C.K., et al., beta-Amyloid stimulation of microglia and monocytes results in TNFalphadependent expression of inducible nitric oxide synthase and neuronal apoptosis. J Neurosci, 2001. 21(4): p. 1179-88.

36. Wang, W.F., et al., Association of interleukin-I beta and receptor antagonist gene polymorphisms with late onset Alzheimer's disease in Taiwan Chinese. Eur J Neurol, 2005. 12(8): p. 609-13.

37. Flood, D.G. and P.D. Coleman, Failed compensatory dendritic growth as a pathophysiological process in Alzheimer's disease. Can J Neurol Sci, 1986. 13(4 Suppl): p. 475-9.

38. Hoff, S.F., et al., A new type of lesion-induced synaptogenesis: II. The effect of aging on synaptic turnover in non-denervated zones. Brain Res, 1981. 222(1): p. 15-27.

39. Masliah, E., et al., Synaptic and neuritic alterations during the progression of Alzheimer's disease. Neurosci Lett, 1994. 174(1): p. 67-72.

40. Rabinovici, G.D., et al., 11C-PIB PET imaging in Alzheimer disease and frontotemporal lobar degeneration. Neurology, 2007. 68(15): p. 1205-12.

41. Behrman, E.J. and V. Gopalan, Cholesterol and plants. J. Chem. Educ., 2005. 82(12): p. 1791.

42. Weete, J.D., et al., Fatty Acids and Sterols of Selected Hyphochytriomycetes and Chytridiomycetes. Experimental mycology 1989. 13: p. 183-195.

43. Olson, R.E., Discovery of the lipoproteins, their role in fat transport and their significance as risk factors. J Nutr, 1998. 128(2 Suppl): p. 439S-443S.

44. Beattie, M.E., et al., Sterol structure determines miscibility versus melting transitions in lipid vesicles. Biophys J, 2005. 89(3): p. 1760-8.

45. Haines, T.H., Do sterols reduce proton and sodium leaks through lipid bilayers? Prog Lipid Res, 2001. 40(4): p. 299-324.

46. Berg, J.M., et al., Biochemistry. 5th ed. Chapter 26: The Biosynthesis of Membrane Lipids and Steroids, ed. S. Moran. 2002, New York: W. H. Freeman and Company.

47. Goldstein, J.L. and M.S. Brown, Regulation of the mevalonate pathway. Nature, 1990. 343(6257): p. 425-30.

48. Lutjohann, D., et al., Profile of cholesterol-related sterols in aged amyloid precursor protein transgenic mouse brain. J Lipid Res, 2002. 43(7): p. 1078-85.

49. Nieweg, K., H. Schaller, and F.W. Pfrieger, Marked differences in cholesterol synthesis between neurons and glial cells from postnatal rats. J Neurochem, 2009. 109(1): p. 125-34.

50. Wender, M., H. Filipek-Wender, and J. Stanislawska, Cholesteryl esters of the brain in demyelinating diseases. Clin Chim Acta, 1974. 54(3): p. 269-75.

51. Dietschy, J.M. and S.D. Turley, Control of cholesterol turnover in the mouse. J Biol Chem, 2002. 277(6): p. 3801-4.

52. Lütjohann, D., T. Vanmierlo, and M. Mulder, Cellular lipid metabolism; Chapter 5: Cholesterol trafficking in the brain pp 131-155. 1st ed, ed. C. Ehnholm. 2009, Berlin Heidelberg: Springer Berlin Heidelberg. 131-155. 
53. Edmond, J., et al., Dietary cholesterol and the origin of cholesterol in the brain of developing rats. $\mathrm{J}$ Nutr, 1991. 121(9): p. 1323-30.

54. Bjorkhem, I. and S. Meaney, Brain cholesterol: long secret life behind a barrier. Arterioscler Thromb Vasc Biol, 2004. 24(5): p. 806-15.

55. Dietschy, J.M. and S.D. Turley, Cholesterol metabolism in the brain. Curr Opin Lipidol, 2001. 12(2): p. $105-12$.

56. Spady, D.K. and J.M. Dietschy, Sterol synthesis in vivo in 18 tissues of the squirrel monkey, guinea pig, rabbit, hamster, and rat. J Lipid Res, 1983. 24(3): p. 303-15.

57. Ando, S., et al., Turnover of myelin lipids in aging brain. Neurochem Res, 2003. 28(1): p. 5-13.

58. Smith, M.E., The turnover of myelin in the adult rat. Biochim Biophys Acta, 1968. 164(2): p. $285-93$.

59. Spohn, M. and A.N. Davison, Cholesterol metabolism in myelin and other subcellular fractions of rat brain. J Lipid Res, 1972. 13(5): p. 563-70.

60. Davison, A.N., Brain sterol metabolism. Adv Lipid Res, 1965. 3: p. 171-96.

61. Muse, E.D., et al., Parameters related to lipid metabolism as markers of myelination in mouse brain. $\mathrm{J}$ Neurochem, 2001. 76(1): p. 77-86.

62. Xie, C., et al., Quantitation of two pathways for cholesterol excretion from the brain in normal mice and mice with neurodegeneration. J Lipid Res, 2003. 44(9): p. 1780-9.

63. Pfrieger, F.W., Cholesterol homeostasis and function in neurons of the central nervous system. Cell Mol Life Sci, 2003. 60(6): p. 1158-71.

64. Pfrieger, F.W., Outsourcing in the brain: do neurons depend on cholesterol delivery by astrocytes? Bioessays, 2003. 25(1): p. 72-8.

65. Vance, J.E., et al., Evidence that the major membrane lipids, except cholesterol, are made in axons of cultured rat sympathetic neurons. J Neurochem, 1994. 62(1): p. 329-37.

66. Wahrle, S.E., et al., ABCA1 is required for normal central nervous system ApoE levels and for lipidation of astrocyte-secreted apoE. J Biol Chem, 2004. 279(39): p. 40987-93.

67. Wang, N., et al., ATP-binding cassette transporters G1 and G4 mediate cholesterol and desmosterol efflux to HDL and regulate sterol accumulation in the brain. Faseb J, 2008. 22(4): p. 1073-82.

68. Gong, J.S., et al., Apolipoprotein E (ApoE) isoform-dependent lipid release from astrocytes prepared from human ApoE3 and ApoE4 knock-in mice. J Biol Chem, 2002. 277(33): p. 29919-26.

69. Legleiter, J., et al., In situ AFM studies of astrocyte-secreted apolipoprotein E- and J-containing lipoproteins. J Colloid Interface Sci, 2004. 278(1): p. 96-106.

70. Chintagari, N.R., et al., Effect of cholesterol depletion on exocytosis of alveolar type II cells. Am J Respir Cell Mol Biol, 2006. 34(6): p. 677-87.

71. Toledo, J.D., et al., Cholesterol flux between lipid vesicles and apolipoprotein AI discs of variable size and composition. Arch Biochem Biophys, 2000. 380(1): p. 63-70.

72. Guo, J., et al., Effects of cholesterol levels on the excitability of rat hippocampal neurons. Mol Membr Biol, 2008. 25(3): p. 216-23.

73. Lund, E.G., J.M. Guileyardo, and D.W. Russell, cDNA cloning of cholesterol 24-hydroxylase, a mediator of cholesterol homeostasis in the brain. Proc Natl Acad Sci U S A, 1999. 96(13): p. 7238-43.

74. Russell, D.W., et al., Cholesterol 24-hydroxylase: an enzyme of cholesterol turnover in the brain. Annu Rev Biochem, 2009. 78: p. 1017-40.

75. Bjorkhem, I., et al., Importance of a novel oxidative mechanism for elimination of brain cholesterol. Turnover of cholesterol and 24(S)-hydroxycholesterol in rat brain as measured with 1802 techniques in vivo and in vitro. J Biol Chem, 1997. 272(48): p. 30178-84.

76. Lutjohann, D., et al., Cholesterol homeostasis in human brain: evidence for an age-dependent flux of 24S-hydroxycholesterol from the brain into the circulation. Proc Natl Acad Sci U S A, 1996. 93(18): p. 9799-804.

77. Panzenboeck, U., et al., ABCA1 and scavenger receptor class B, type I, are modulators of reverse sterol transport at an in vitro blood-brain barrier constituted of porcine brain capillary endothelial cells. J Biol Chem, 2002. 277(45): p. 42781-9.

78. Kessel, A., N. Ben-Tal, and S. May, Interactions of cholesterol with lipid bilayers: the preferred configuration and fluctuations. Biophys J, 2001. 81(2): p. 643-58.

79. Rebeck, G.W., Cholesterol efflux as a critical component of Alzheimer's disease pathogenesis. J Mol Neurosci, 2004. 23(3): p. 219-24.

80. Shafaati, M., et al., Levels of ApoE in cerebrospinal fluid are correlated with Tau and 24Shydroxycholesterol in patients with cognitive disorders. Neurosci Lett, 2007. 425(2): p. 78-82. 
81. Abildayeva, K., et al., 24(S)-Hydroxycholesterol Participates in a Liver X Receptor-controlled Pathway in Astrocytes That Regulates Apolipoprotein E-mediated Cholesterol Efflux. J Biol Chem, 2006. 281(18): p. 12799-12808.

82. Goldstein, J.L., R.A. DeBose-Boyd, and M.S. Brown, Protein sensors for membrane sterols. Cell, 2006. 124(1): p. 35-46.

83. Eckert, G.P., et al., Regulation of central nervous system cholesterol homeostasis by the liver $X$ receptor agonist TO-901317. Neurosci Lett, 2007. 423(1): p. 47-52.

84. Zelcer, N. and P. Tontonoz, Liver X receptors as integrators of metabolic and inflammatory signaling. J Clin Invest, 2006. 116(3): p. 607-14.

85. Whitney, K.D., et al., Regulation of cholesterol homeostasis by the liver X receptors in the central nervous system. Mol Endocrinol, 2002. 16(6): p. 1378-85.

86. Janowski, B.A., et al., An oxysterol signalling pathway mediated by the nuclear receptor LXR alpha. Nature, 1996. 383(6602): p. 728-31.

87. Ikonen, E., Cellular cholesterol trafficking and compartmentalization. Nat Rev Mol Cell Biol, 2008. 9(2): p. 125-38.

88. Song, B.L., N.B. Javitt, and R.A. DeBose-Boyd, Insig-mediated degradation of HMG CoA reductase stimulated by lanosterol, an intermediate in the synthesis of cholesterol. Cell Metab, 2005. 1(3): p. 17989.

89. $\mathrm{Bu}, \mathrm{G}$., et al., Nerve growth factor induces rapid increases in functional cell surface low density lipoprotein receptor-related protein. J Biol Chem, 1998. 273(21): p. 13359-65.

90. Jaeger, S. and C.U. Pietrzik, Functional role of lipoprotein receptors in Alzheimer's disease. Curr Alzheimer Res, 2008. 5(1): p. 15-25.

91. $\mathrm{Bu}, \mathrm{G}$., et al., Subcellular localization and endocytic function of low density lipoprotein receptorrelated protein in human glioblastoma cells. J Biol Chem, 1994. 269(47): p. 29874-82.

92. Rebeck, G.W., et al., Multiple, diverse senile plaque-associated proteins are ligands of an apolipoprotein E receptor, the alpha 2-macroglobulin receptor/low-density-lipoprotein receptorrelated protein. Ann Neurol, 1995. 37(2): p. 211-7.

93. Rebeck, G.W., et al., Apolipoprotein E in sporadic Alzheimer's disease: allelic variation and receptor interactions. Neuron, 1993. 11(4): p. 575-80.

94. Nathan, B.P., et al., Apolipoprotein E4 inhibits, and apolipoprotein E3 promotes neurite outgrowth in cultured adult mouse cortical neurons through the low-density lipoprotein receptor-related protein. Brain Res, 2002. 928(1-2): p. 96-105.

95. Zerbinatti, C.V. and G. Bu, LRP and Alzheimer's disease. Rev Neurosci, 2005. 16(2): p. 123-35.

96. Zerbinatti, C.V., et al., Apolipoprotein $E$ and low density lipoprotein receptor-related protein facilitate intraneuronal Abeta42 accumulation in amyloid model mice. J Biol Chem, 2006. 281(47): p. 36180-6.

97. Zerbinatti, C.V., et al., Increased soluble amyloid-beta peptide and memory deficits in amyloid model mice overexpressing the low-density lipoprotein receptor-related protein. Proc Natl Acad Sci U S A, 2004. 101(4): p. 1075-80.

98. Offe, K., et al., The lipoprotein receptor LR11 regulates amyloid beta production and amyloid precursor protein traffic in endosomal compartments. J Neurosci, 2006. 26(5): p. 1596-603.

99. $\quad$ Posse De Chaves, E.I., et al., Uptake of lipoproteins for axonal growth of sympathetic neurons. J Biol Chem, 2000. 275(26): p. 19883-90.

100. Salen, G., E.H. Ahrens, Jr., and S.M. Grundy, Metabolism of beta-sitosterol in man. J Clin Invest, 1970. 49(5): p. 952-67.

101. Subbiah, M., Significance of dietary plant sterols in man and experimental animals. Mayo Clin Proc 1971. 46: p. 549.

102. Pollak, O.J. and D. Kritchevsky, Sitosterol. Monogr Atheroscler, 1981. 10: p. 1-219.

103. Salen, G., et al., Increased plasma cholestanol and 5 alpha-saturated plant sterol derivatives in subjects with sitosterolemia and xanthomatosis. J Lipid Res, 1985. 26(2): p. 203-9.

104. Ostlund, R.E., Jr., et al., Gastrointestinal absorption and plasma kinetics of soy Delta(5)-phytosterols and phytostanols in humans. Am J Physiol Endocrinol Metab, 2002. 282(4): p. E911-6.

105. Heinemann, T., G. Axtmann, and K. von Bergmann, Comparison of intestinal absorption of cholesterol with different plant sterols in man. Eur J Clin Invest, 1993. 23(12): p. 827-31.

106. Lutjohann, D., et al., Sterol absorption and sterol balance in phytosterolemia evaluated by deuteriumlabeled sterols: effect of sitostanol treatment. J Lipid Res, 1995. 36(8): p. 1763-73.

107. Altmann, S.W., et al., Niemann-Pick C1 Like 1 protein is critical for intestinal cholesterol absorption. Science, 2004. 303(5661): p. 1201-4. 
108. Davis, H.R., Jr., et al., Niemann-Pick C1 Like 1 (NPC1L1) is the intestinal phytosterol and cholesterol transporter and a key modulator of whole-body cholesterol homeostasis. J Biol Chem, 2004. 279(32): p. 33586-92.

109. Liu, J., et al., Investigating the allosterism of acyl-CoA:cholesterol acyltransferase (ACAT) by using various sterols: in vitro and intact cell studies. Biochem J, 2005. 391(Pt 2): p. 389-97.

110. Calpe-Berdiel, L., J.C. Escola-Gil, and F. Blanco-Vaca, New insights into the molecular actions of plant sterols and stanols in cholesterol metabolism. Atherosclerosis, 2008.

111. Iqbal, J., K. Anwar, and M.M. Hussain, Multiple, independently regulated pathways of cholesterol transport across the intestinal epithelial cells. J Biol Chem, 2003. 278(34): p. 31610-20.

112. Igel, M., et al., Comparison of the intestinal uptake of cholesterol, plant sterols, and stanols in mice. $\mathrm{J}$ Lipid Res, 2003. 44(3): p. 533-8.

113. Nordby, G. and K.R. Norum, Substrate specificity of lecithin:cholesterol acyltransferase. Esterification of desmosterol, b-sitosterol, and cholecalciferol in human plasma. Scand J Clin Lab Invest, 1975. 35(7): p. 677-82.

114. Boberg, K.M. and S. Skrede, Content of sitosterol, cholestanol, and cholesterol in very low density lipoproteins of rat liver perfusate. Scand J Gastroenterol, 1988. 23(4): p. 442-8.

115. Fricke, C.B., et al., Increased plant sterol and stanol levels in brain of Watanabe rabbits fed rapeseed oil derived plant sterol or stanol esters. Br J Nutr, 2007. 98(5): p. 890-9.

116. Weingartner, O., et al., Vascular effects of diet supplementation with plant sterols. J Am Coll Cardiol, 2008. 51(16): p. 1553-61.

117. Jansen, P.J., et al., Dietary plant sterols accumulate in the brain. Biochim Biophys Acta, 2006.

118. Tint, G.S., et al., Markedly increased tissue concentrations of 7-dehydrocholesterol combined with low levels of cholesterol are characteristic of the Smith-Lemli-Opitz syndrome. J Lipid Res, 1995. 36(1): p. 89-95.

119. Repa, J.J., et al., Liver X receptor activation enhances cholesterol loss from the brain, decreases neuroinflammation, and increases survival of the NPC1 mouse. J Neurosci, 2007. 27(52): p. 14470-80.

120. Antoine, J.C., et al., Pathology of roots, spinal cord and brainstem in syringomyelia-like syndrome of Tangier disease. J Neurol Sci, 1991. 106(2): p. 179-85.

121. Salen, G., et al., Lethal atherosclerosis associated with abnormal plasma and tissue sterol composition in sitosterolemia with xanthomatosis. J Lipid Res, 1985. 26(9): p. 1126-33.

122. Plosch, T., et al., Sitosterolemia in ABC-transporter G5-deficient mice is aggravated on activation of the liver-X receptor. Gastroenterology, 2004. 126(1): p. 290-300.

123. Simons, K. and W.L. Vaz, Model systems, lipid rafts, and cell membranes. Annu Rev Biophys Biomol Struct, 2004. 33: p. 269-95.

124. Pike, L.J., Lipid rafts: heterogeneity on the high seas. Biochem J, 2004. 378(Pt 2): p. 281-92.

125. Ledesma, M.D. and C.G. Dotti, Amyloid excess in Alzheimer's disease: what is cholesterol to be blamed for? FEBS Lett, 2006. 580(23): p. 5525-32.

126. Ahmed, S.N., D.A. Brown, and E. London, On the origin of sphingolipid/cholesterol-rich detergentinsoluble cell membranes: physiological concentrations of cholesterol and sphingolipid induce formation of a detergent-insoluble, liquid-ordered lipid phase in model membranes. Biochemistry, 1997. 36(36): p. 10944-53.

127. Baumgart, T., S.T. Hess, and W.W. Webb, Imaging coexisting fluid domains in biomembrane models coupling curvature and line tension. Nature, 2003. 425(6960): p. 821-4.

128. Edidin, M., The state of lipid rafts: from model membranes to cells. Annu Rev Biophys Biomol Struct, 2003. 32: p. 257-83.

129. Niemela, P.S., et al., Assessing the nature of lipid raft membranes. PLoS Comput Biol, 2007. 3(2): p. e34.

130. Simons, K. and E. Ikonen, Functional rafts in cell membranes. Nature, 1997. 387(6633): p. 569-72.

131. Friedrichson, T. and T.V. Kurzchalia, Microdomains of GPI-anchored proteins in living cells revealed by crosslinking. Nature, 1998. 394(6695): p. 802-5.

132. Varma, R. and S. Mayor, GPI-anchored proteins are organized in submicron domains at the cell surface. Nature, 1998. 394(6695): p. 798-801.

133. Cheng, Z.J., et al., Membrane microdomains, caveolae, and caveolar endocytosis of sphingolipids. Mol Membr Biol, 2006. 23(1): p. 101-10.

134. Ostrom, R.S. and X. Liu, Detergent and detergent-free methods to define lipid rafts and caveolae. Methods Mol Biol, 2007. 400: p. 459-68. 
135. Jarvik, G.P., et al., Interactions of apolipoprotein E genotype, total cholesterol level, age, and sex in prediction of Alzheimer's disease: a case-control study. Neurology, 1995. 45(6): p. 1092-6.

136. Kivipelto, M., et al., Apolipoprotein E epsilon4 allele, elevated midlife total cholesterol level, and high midlife systolic blood pressure are independent risk factors for late-life Alzheimer disease. Ann Intern Med, 2002. 137(3): p. 149-55.

137. Pappolla, M.A., et al., Mild hypercholesterolemia is an early risk factor for the development of Alzheimer amyloid pathology. Neurology, 2003. 61(2): p. 199-205.

138. Shie, F.S., et al., Diet-induced hypercholesterolemia enhances brain A beta accumulation in transgenic mice. Neuroreport, 2002. 13(4): p. 455-9.

139. Sparks, D.L., Intraneuronal beta-amyloid immunoreactivity in the CNS. Neurobiol Aging, 1996. 17(2): p. 291-9.

140. Wirths, O., et al., Decreased plasma cholesterol levels during aging in transgenic mouse models of Alzheimer's disease. Exp Gerontol, 2006. 41(2): p. 220-4.

141. Beach, T.G., et al., Circle of Willis atherosclerosis: association with Alzheimer's disease, neuritic plaques and neurofibrillary tangles. Acta Neuropathol, 2007. 113(1): p. 13-21.

142. Bjorkhem, I., Crossing the barrier: oxysterols as cholesterol transporters and metabolic modulators in the brain. J Intern Med, 2006. 260(6): p. 493-508.

143. Corder, E.H., et al., Gene dose of apolipoprotein E type 4 allele and the risk of Alzheimer's disease in late onset families. Science, 1993. 261(5123): p. 921-3.

144. Jiang, Q., et al., ApoE promotes the proteolytic degradation of Abeta. Neuron, 2008. 58(5): p. 681-93.

145. Liu, Q., et al., Amyloid precursor protein regulates brain apolipoprotein E and cholesterol metabolism through lipoprotein receptor LRP1. Neuron, 2007. 56(1): p. 66-78.

146. Ye, S., et al., Apolipoprotein (apo) E4 enhances amyloid beta peptide production in cultured neuronal cells: apoE structure as a potential therapeutic target. Proc Natl Acad Sci U S A, 2005. 102(51): p. 18700-5.

147. Deane, R., et al., LRP/amyloid beta-peptide interaction mediates differential brain efflux of Abeta isoforms. Neuron, 2004. 43(3): p. 333-44.

148. Koo, E.H. and S.L. Squazzo, Evidence that production and release of amyloid beta-protein involves the endocytic pathway. J Biol Chem, 1994. 269(26): p. 17386-9.

149. Marzolo, M.P. and G. Bu, Lipoprotein receptors and cholesterol in APP trafficking and proteolytic processing, implications for Alzheimer's disease. Semin Cell Dev Biol, 2009. 20(2): p. 191-200.

150. Laffitte, B.A., et al., LXRs control lipid-inducible expression of the apolipoprotein $E$ gene in macrophages and adipocytes. Proc Natl Acad Sci U S A, 2001. 98(2): p. 507-12.

151. Wahrle, S.E., et al., Deletion of Abcal increases Abeta deposition in the PDAPP transgenic mouse model of Alzheimer disease. J Biol Chem, 2005. 280(52): p. 43236-42.

152. Wahrle, S.E., et al., Overexpression of ABCA1 reduces amyloid deposition in the PDAPP mouse model of Alzheimer disease. J Clin Invest, 2008. 118(2): p. 671-82.

153. Crameri, A., et al., The role of seladin-1/DHCR24 in cholesterol biosynthesis, APP processing and Abeta generation in vivo. Embo J, 2006. 25(2): p. 432-43.

154. Riddell, D.R., et al., The LXR agonist TO901317 selectively lowers hippocampal Abeta42 and improves memory in the Tg2576 mouse model of Alzheimer's disease. Mol Cell Neurosci, 2007. 34(4): p. 621-8.

155. Koldamova, R.P., et al., The liver X receptor ligand T0901317 decreases amyloid beta production in vitro and in a mouse model of Alzheimer's disease. J Biol Chem, 2005. 280(6): p. 4079-88.

156. Lefterov, I., et al., Expression profiling in APP23 mouse brain: inhibition of Abeta amyloidosis and inflammation in response to LXR agonist treatment. Mol Neurodegener, 2007. 2(1): p. 20.

157. Fassbender, K., et al., Simvastatin strongly reduces levels of Alzheimer's disease beta-amyloid peptides Abeta 42 and Abeta 40 in vitro and in vivo. Proc Natl Acad Sci U S A, 2001. 98(10): p. 5856-61.

158. Zandi, P.P., et al., Do statins reduce risk of incident dementia and Alzheimer disease? The Cache County Study. Arch Gen Psychiatry, 2005. 62(2): p. 217-24.

159. Li, G., et al., Statin therapy and risk of dementia in the elderly: a community-based prospective cohort study. Neurology, 2004. 63(9): p. 1624-8.

160. Stefani, M. and G. Liguri, Cholesterol in Alzheimer's disease: unresolved questions. Curr Alzheimer Res, 2009. 6(1): p. 15-29.

161. Cecchi, C., et al., Seladin-1/DHCR24 protects neuroblastoma cells against Abeta toxicity by increasing membrane cholesterol content. J Cell Mol Med, 2008. 12(5B): p. 1990-2002. 
162. Sun, Y.X., et al., Pravastatin inhibits pro-inflammatory effects of Alzheimer's peptide Abeta(1-42) in glioma cell culture in vitro. Pharmacol Res, 2003. 47(2): p. 119-26.

163. Zhou, Y., et al., Geranylgeranyl pyrophosphate stimulates gamma-secretase to increase the generation of Abeta and APP-CTFgamma. Faseb J, 2008. 22(1): p. 47-54.

164. Simons, M., et al., Cholesterol depletion inhibits the generation of beta-amyloid in hippocampal neurons. Proc Natl Acad Sci U S A, 1998. 95(11): p. 6460-4.

165. Wolozin, B., A fluid connection: cholesterol and Abeta. Proc Natl Acad Sci U S A, 2001. 98(10): p. 5371-3.

166. Spasic, D. and W. Annaert, Building gamma-secretase: the bits and pieces. J Cell Sci, 2008. 121(Pt 4): p. 413-20.

167. Vetrivel, K.S., et al., Spatial segregation of gamma-secretase and substrates in distinct membrane domains. J Biol Chem, 2005. 280(27): p. 25892-900.

168. Ehehalt, R., et al., Amyloidogenic processing of the Alzheimer beta-amyloid precursor protein depends on lipid rafts. J Cell Biol, 2003. 160(1): p. 113-23.

169. Ledesma, M.D., et al., Raft disorganization leads to reduced plasmin activity in Alzheimer's disease brains. EMBO Rep, 2003. 4(12): p. 1190-6.

170. Abad-Rodriguez, J., et al., Neuronal membrane cholesterol loss enhances amyloid peptide generation. J Cell Biol, 2004. 167(5): p. 953-60.

171. Puglielli, L., et al., Acyl-coenzyme A: cholesterol acyltransferase modulates the generation of the amyloid beta-peptide. Nat Cell Biol, 2001. 3(10): p. 905-12.

172. Kotti, T., et al., Biphasic requirement for geranylgeraniol in hippocampal long-term potentiation. Proc Natl Acad Sci U S A, 2008. 105(32): p. 11394-9. 



\section{Chapter II}

\section{Alterations in Brain Cholesterol Metabolism in the APPSLXPS1mut mouse, a model for Alzheimer's Disease.}




\section{Chapter II}

\section{Alterations in Brain Cholesterol Metabolism in the APPSLxPS1mut mouse, a Model for Alzheimer's Disease.}

Tim Vanmierlo ${ }^{\mathrm{a}, \mathrm{b}, \mathrm{e}}$, Vincent W. Bloks ${ }^{\mathrm{c}}$, Leonie C. van Vark- van der Zee ${ }^{\mathrm{d}}$, Kris Rutten $^{\mathrm{a}, \mathrm{e}}$, Anja Kerksiek ${ }^{\mathrm{b}}$, Silvia Friedrichs ${ }^{\mathrm{b}}$, Eric Sijbrands ${ }^{\mathrm{d}}$, Harry W. Steinbusch $^{\mathrm{a}, \mathrm{e}}$, Folkert Kuipers ${ }^{\mathrm{c}}$, Dieter Lütjohann ${ }^{\mathrm{b}, \mathrm{e}}$, Monique Mulder ${ }^{\mathrm{a}, \mathrm{d}, \mathrm{e}}$

${ }^{a}$ Department of Neuroscience, Maastricht University, 6200 MD Maastricht, The Netherlands; ${ }^{b}$ Institute of Clinical Chemistry and Pharmacology, University of Bonn, 53105 Bonn, Germany; ${ }^{\mathrm{c}}$ Department of Pediatrics, University Medical Center Groningen, 9713 GZ. Groningen, The Netherlands; ${ }^{\mathrm{d}}$ Department of Internal Medicine, Division of Pharmacology, Vascular and Metabolic Diseases, Erasmus Medical Center, 3015 CE Rotterdam, The Netherlands; ${ }^{\text {e }}$ EURON (European Graduate School for Neuroscience

J Alzheimers Dis, 2010. 19(1): p. 117-27 


\section{Abstract}

Disturbances in cerebral cholesterol metabolism have been implicated in the pathogenesis of Alzheimer's disease (AD). Here, we provide evidence that alterations in brain cholesterol homeostasis also can be a consequence of disease progression. We found that APPSLxPS1mut mice, at the age of 9 months when AD-like pathology starts to develop, display increased levels of the cholesterol precursor desmosterol and of the cholesterol metabolite 27-hydroxy $(\mathrm{OH})$ cholesterol in their cerebellum in comparison with wild-type controls. At the age of 21 months, when APPSLxPS1mut brain contains abundant amyloid deposits, desmosterol levels had further increased ( $>200 \%$ in comparison with wild-type mice) in all brain regions examined. 24(S)-OHcholesterol levels were increased in hippocampus and cerebellum of the APPSLXPS1mut mice, while 27-OHcholesterol levels were increased in cerebellum exclusively. Brain cholesterol levels remained unaffected. In line with the fact that desmosterol and 24(S)-OHcholesterol are Liver X Receptor (LXR) activators, the LXR-target genes Abcal and Apoc1 were upregulated predominantly in hippocampus of APPSLxPS1mut mice at both ages evaluated. The reduced expression of the enzyme that converts desmosterol into cholesterol, the Selective AD indicator 1 gene (Seladin-1/Dhcr24), in both cortex and cerebellum may underlie the increased desmosterol levels in 21 month-old APPSLxPS1mut mice.

\section{Introduction}

Alzheimer's disease (AD) is a slowly progressive neurodegenerative disease leading to a gradual loss of higher cognitive functions such as short-term and spatial memory. At present, there is no all-embracing effective therapy. Deposition of amyloid- $\beta(A \beta)$, cleaved from its larger $A \beta$-precursor protein (APP), in senile plaques is a major neuropathological hallmark of $\mathrm{AD}$ [1]. Neither the precise physiological functions of APP or A $\beta$, nor their roles in the development of AD are fully understood yet. Processing of APP is affected by cholesterol [2-4]. Of all organs in the human body, the brain has the highest content of free cholesterol. Cholesterol homeostasis in the central nervous system is maintained largely independently of that in the remainder of the body. Therefore, in the brain all cholesterol is synthesized endogenously and no cholesterol is retrieved from the circulation. Brain cholesterol turnover is low, i.e. $<1 \% /$ day. Since cholesterol cannot be degraded and since high levels of free cholesterol are toxic, in particular to neurons, excess cholesterol is secreted from the brain into the circulation. About 
$60 \%$ is secreted in the form of the brain-specific metabolite 24(S)-OHcholesterol and the remaining $40 \%$ via yet unknown pathways, which may involve apolipoprotein E (ApoE) and ATP binding cassette (ABC)A1 [5-8].

Maintenance of brain cholesterol homeostasis involves complex processes (Fig. 1). As previously shown, aberrations in cholesterol metabolism within the brain can lead to severe neurological diseases [9, 10]. Accumulated epidemiological and experimental evidence indicates involvement of cholesterol in the development and progression of $\mathrm{AD}$ [11-13]. This is supported by the fact that apoE4, one of the three common forms of ApoE in humans, is associated with an increased risk of AD [14]. ApoE is best known for its role in cholesterol transport in the circulation. Moreover, the risk of $\mathrm{AD}$ increased with high circulating cholesterol levels in middle aged subjects, while the use of statins, cholesterol synthesis inhibitors, was found to reduce the risk [15-17]. In addition, the deposition of $A \beta$ in the brain was increased with increasing plasma cholesterol levels and with a high fat intake in AD animal models $[18,19]$. In vitro, alterations in intracellular levels or distribution of cholesterol were found to affect the generation of $A \beta$ from its precursor protein $[2$, $4]$, but on the other hand $A \beta$ was also found to modulate cholesterol synthesis [20, 21]. Thus an increasing amount of evidence points at a mechanistic link between cerebral cholesterol metabolism and the deposition of $\mathrm{A} \beta[22,23]$. The aim of the present study was to assess whether alterations in brain cholesterol metabolism occur as a consequence of disease progression in APPSLxPS1mut mice, a model for AD.

In contrast to the commonly used AD-models that overexpress mutants of the human APP specifically in neurons several-fold [24], these mice carry a humanized APP sequence, that contains both the Swedish and the London mutation under the control of the endogenous murine APP promoter [25]. Therefore, expression of APPSL is not restricted to neurons but comparable to that of murine-APP, and the levels of APPSL are in the physiological range. When crossed with PS1M146Ltransgenic mice, amyloid is deposited predominantly in the cortex and in the hippocampus from the age of 6-9 months onwards with a steep increase from the age of 18 months [25]. This model has been suggested to be more "human-like" than other murine AD models that overexpress a human APP mutant. Our results revealed the occurrence of specific abnormalities in brain cholesterol metabolism in APPSLxPS1mut mice, indicating that these can be a consequence of disease progression. 
Mevalonate Pathway (Rate limiting enzyme: Hmgcr)

HO

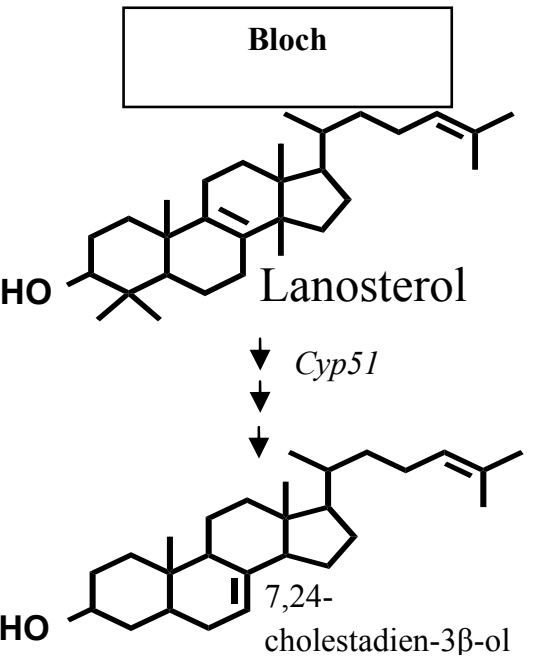

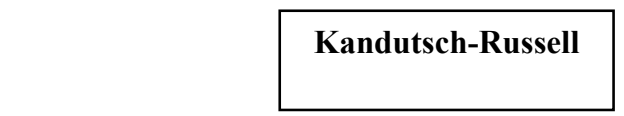

Dhcr24/

$\stackrel{(\text { Seladin1) }}{\longrightarrow}$

HO

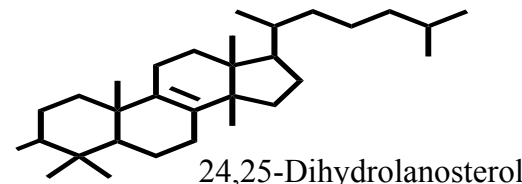

Dhcr24/

(Seladin1)
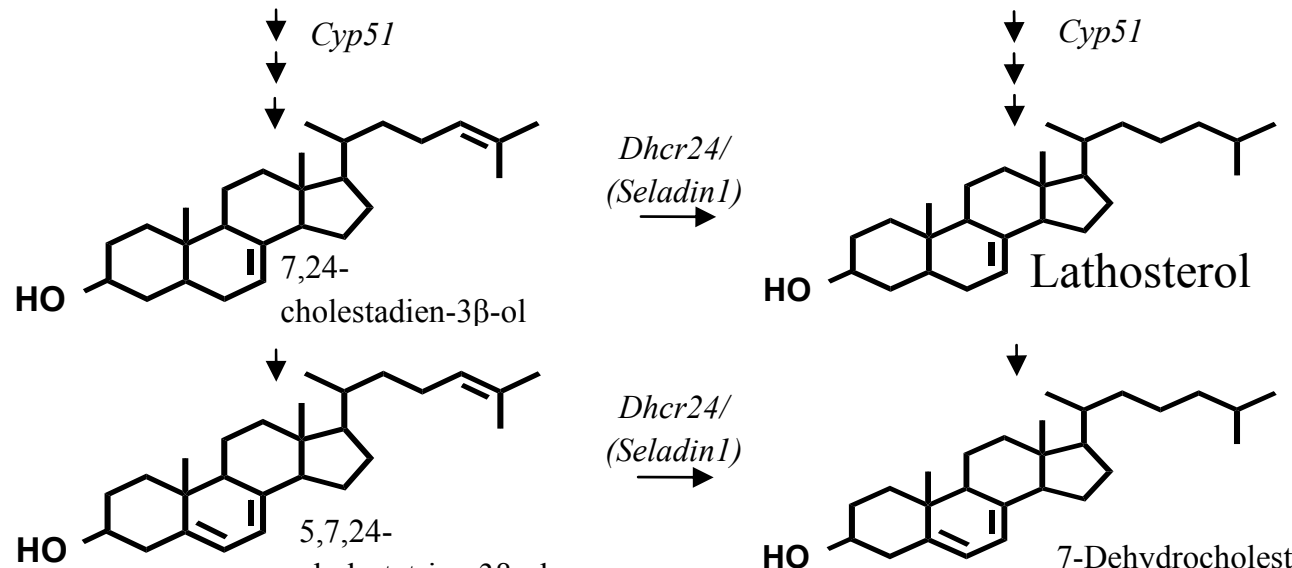

Dhcr24/

(Seladin1)

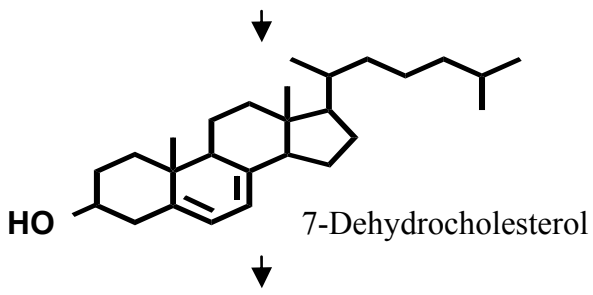

HO

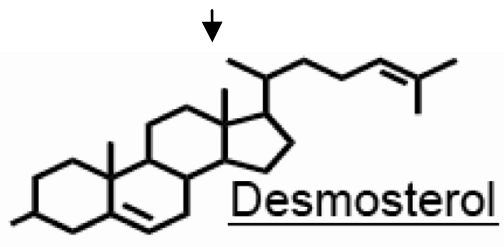

Dhcr24/

(Seladin1)
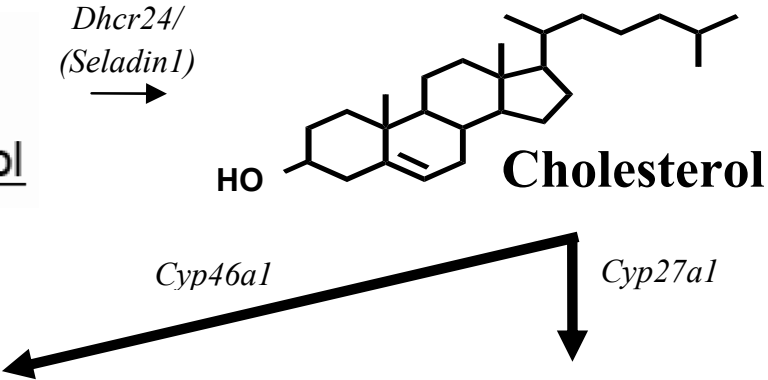

24(S)-OHcholesterol

27-OHcholesterol

Fig.1. The main cholesterol post-mevalonate synthesis and metabolizing pathways.

On the left hand side, the main players in the Bloch pathway are shown together with the genes coding for the necessary enzymes involved of the conversion. On the right hand side, the key steps of the Kandutsch-Russell pathway are shown in the formation of cholesterol. The switch from the Bloch pathway towards the Kandutsch-Russel pathway is mediated by the enzyme encoded by Dhcr24 (Seladin1). Cholesterol can be metabolized into oxysterols from which 24(S)OHcholesterol and 27-OHcholesterol are shown. Important enzymes involved are displayed in Italics. 


\section{Materials and Methods}

Animals

All experimental procedures were approved by the local ethical committee of the Maastricht University for animal experiments according to the Netherlands' governmental guidelines. Mice with gene-targeted expression of the APP mutant encoding the Swedish/London-FAD mutations (APPSL) were generated as described by Köhler et al. [25]. The APPSL mice were crossbred with PS1(M146L) transgenic mice [26] to generate APPSLxPS1mut mice on a mixed Sv129, Black Swiss and C57BL/6NCrl background [25]. Subsequently, mice were backcrossed 6 times with C57BL/6NCrl mice to finally obtain APPSLxPS1mut mice on a steady C57BL/6NCrl background. C57BL/6NCrl mice were obtained from Charles River Laboratories (L'Arbresle, France) animals will further on be referred to as wild-type controls. Animals had access to food and water ad libitum and were kept on a normal 12h day/night rhythm (lights on 7AM; lights off 7PM). All experiments were performed using male APPSLxPS1mut and C57BL/6NCrl mice.

\section{Analyses}

\section{Sample preparation}

The mice were anaesthetized IP with ketamine/xylazine $(100 \mathrm{mg} / \mathrm{kg}$ ketamine and 5 $\mathrm{mg} / \mathrm{kg}$ xylazine) and subsequently perfused with phosphate buffered saline (PBS) $\left(137 \mathrm{mM} \mathrm{NaCl} ; 2.7 \mathrm{mM} \mathrm{KCl} ; 6.5 \mathrm{mM} \mathrm{Na}_{2} \mathrm{HPO}_{4} ; 1.5 \mathrm{mM} \mathrm{KH}_{2} \mathrm{PO}_{4} ; \mathrm{pH}=7.3\right)$. Blood was collected prior to perfusion by puncturing of the heart and spun $10 \mathrm{~min}$ at 1400rpm to obtain serum, which was snap frozen in liquid nitrogen. Brains were divided into two hemispheres that were subdivided into hippocampus (HC), cortex (CTX), and cerebellum (CB). These were either used for sterol analyses or for Quantitative Real Time Polymerase Chain Reaction (Q-RT-PCR).

\section{Sterol profile determination}

Preceding the analysis, samples were spun in a speed vacuum dryer (12mbar) (Savant AES 1000) for 24h to relate individual sterol concentrations to their dry weight. The sterols were extracted from the dried tissue by placing them in a $2 \mathrm{ml}$ mixture of chloroform-methanol $(2: 1)$ for $24 \mathrm{~h}$ at $4^{\circ} \mathrm{C}$. Sterol levels were determined by gas chromatography/mass spectrometry (GC/MS) as described previously [27, 28].

\section{$R N A$ isolation and RT-procedures}

Total RNA from cerebellum, hippocampus and cortex was isolated using the Trizol Reagent method (Invitrogen, Breda, The Netherlands) according to the manufacturer instructions. Integrity of RNA was checked by a $1.5 \%$ agarose gel 
electrophoresis, and RNA concentrations were measured spectrophotometrically (NanoDrop, Witec AG, Littau, Germany). cDNA was synthesized from a $1 \mu \mathrm{g}$ mRNA input quantity using the Bio-Rad iScript cDNA synthesis kit (Bio-Rad, Veenendaal, the Netherlands) according to the manufacturer's instructions. cDNA equivalent to $10 \mathrm{ng}$ of total RNA was amplified using the Q-RT-PCR core kit (Eurogentec, Seraing, Belgium) according to the manufacturers protocol. Q-RTPCR was performed using an Applied Biosystems 7700 sequence detector according to the manufacturer's instructions as described before [29], with modifications [30]. Primer oligomers (Invitrogen, Breda, The Netherlands) and fluorogenic probes, labeled with 6-carboxyfluorescein (5'- FAM) and 6carboxytetramethylrhodamine (3'- TAMRA), were purchased by Eurogentec (Seraing, Belgium). Primer and probe sequences are published and available online at RTprimerDB and are shown in Table I. All expression data were subsequently normalized for $18 \mathrm{~S}$ rRNA, which was analyzed in a separate run.

Table I: Primer and probe sequences applied in Q-RT-PCR assay

\begin{tabular}{|c|c|c|c|}
\hline Gene & 5' Forward primer & 3'Reverse primer & probe \\
\hline Dhcr24 & $\begin{array}{l}\text { AGCTTTGCTGAACTC } \\
\text { CATTGG }\end{array}$ & $\begin{array}{l}\text { CCCCCCACTGTGAGGT } \\
\text { CAT }\end{array}$ & TGGACCCTGCCTGTGTTGCCTGA \\
\hline Cyp51 & $\begin{array}{l}\text { CCACGCTGCCTGGCT } \\
\text { ATT }\end{array}$ & $\begin{array}{l}\text { CTATCCCTGCGCCTGA } \\
\text { AACT }\end{array}$ & CCAGCTTGGCTGCCTCTGCCA \\
\hline Hmger & $\begin{array}{l}\text { CCGGCAACAACAAG } \\
\text { ATCTGTG }\end{array}$ & $\begin{array}{l}\text { ATGTACAGGATGGCG } \\
\text { ATGCA }\end{array}$ & TGTCGCTGCTCAGCACGTCCTCTTC \\
\hline Cyp27a1 & $\begin{array}{l}\text { GCCTTGCACAAGGA } \\
\text { AGTGACT }\end{array}$ & $\begin{array}{l}\text { CGCAGGGTCTCCTTAA } \\
\text { TCACA }\end{array}$ & CCCTTCGGGAAGGTGCCCCAG \\
\hline Cyp46a1 & $\begin{array}{l}\text { AACCATCTGGCATTC } \\
\text { ACAGTGA }\end{array}$ & $\begin{array}{l}\text { GGAACCGACAACCTC } \\
\text { ATCCA }\end{array}$ & TCGGCCTGCAGCCTTGCCAC \\
\hline Abcal & $\begin{array}{l}\text { CCCAGAGCAAAAAG } \\
\text { CGACTC }\end{array}$ & $\begin{array}{l}\text { GGTCATCATCACTTTG } \\
\text { GTCCTTG }\end{array}$ & $\begin{array}{l}\text { AGACTACTCTGTCTCTCAGACAACAC } \\
\text { TTGACCAAG }\end{array}$ \\
\hline Abcg1 & $\begin{array}{l}\text { CAAGACCCTTTTGAA } \\
\text { AGGGATCTC }\end{array}$ & $\begin{array}{l}\text { GCCAGAATATTCATGA } \\
\text { GTGTGGAC }\end{array}$ & CCCATGATGGCCACCAGCTCTCC \\
\hline Apoe & $\begin{array}{l}\text { CCTGAACCGCTTCTG } \\
\text { GGATT }\end{array}$ & $\begin{array}{l}\text { GCTCTTCCTGGACCTG } \\
\text { GTCA }\end{array}$ & AAAGCGTCTGCACCCAGCGCAGG \\
\hline Apoc1 & $\begin{array}{l}\text { GGGCAGCCATTGAA } \\
\text { CATATCA }\end{array}$ & $\begin{array}{l}\text { TTGCCAAATGCCTCTG } \\
\text { AGAAC }\end{array}$ & CCCGGGTCTTGGTCAAAATTTCCTTC \\
\hline Srebplc & $\begin{array}{l}\text { GGAGCCATGGATTG } \\
\text { CACATT }\end{array}$ & $\begin{array}{l}\text { CCTGTCTCACCCCCAG } \\
\text { CATA }\end{array}$ & $\begin{array}{l}\text { CAGCTCATCAACAACCAAGACAGTG } \\
\text { ACTTCC }\end{array}$ \\
\hline $18 \mathrm{~S}$ & $\begin{array}{l}\text { CGGCTACCACATCCA } \\
\text { AGGA }\end{array}$ & $\begin{array}{l}\text { CCAATTACAGGGCCTC } \\
\text { GAAA }\end{array}$ & CGCGCAAATTACCCACTCCCGA \\
\hline 11 & were & $h$ & xyfluorescein \\
\hline
\end{tabular}




\section{Statistics}

Statistical analyses were performed using GraphPad Prism 4®. Genotypes were compared for significant differences using the independent sample two tailed student t-test. In the case of low sample size, when normality and Gaussian distribution could not be proven (mRNA expression data), genotypes were compared using the non parametrical Mann Whitney U (MWU) test. Because of the low sample size, mRNA expression data were displayed as scatter dot plots. Significance levels were determined on $\mathrm{P}<0.05^{(*)}, \mathrm{P}<0.01^{(* *)}$ or $\mathrm{P}<0.001^{(* * *)}$. Extreme values were excluded using Dixon's principles of exclusion of extreme values $[31,32]$.

\section{Results}

The APPSLxPS1mut mice appeared healthy and there were no differences in general behavior in comparison with wild-type mice. However, their body weight was significantly higher than that of the wild-type mice at the age of 9 months $(\mathrm{t}(8)=7.72 ; \mathrm{P}<0.001)$. At the age of 21 months the weight difference was not significant anymore $(\mathrm{t}(16)=1.80 ; \mathrm{P}=0.09)$ (Fig. 2). No gross differences in intake of food and water and in the excretion of faeces and urine were detected over a period

Wild type controls

$\square$ APPSLxPS1mut
A
9 MONTHS
B
21 MONTHS
$* * *$
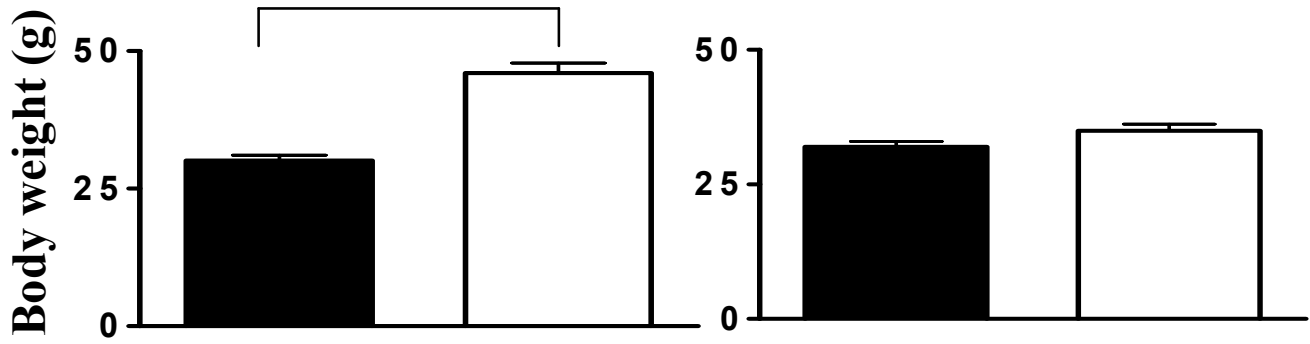

of two days (data not shown).

Fig.2. Body weight of APPSLxPS1mut versus wild-type mice.

At the age of 9 months APPSLxPS1mut displayed a significantly higher body weight than the wild-type mice (A). At 21 months the difference did not reach significance anymore (B). Values are displayed as mean + SEM. $* * *=\mathrm{P}<0.001$ 
Brain, liver and serum sterol profiles of APPSLxPS1mut and wild-type mice of 9 and 21 months old were analyzed using GC/MS. At the age of 9 months, levels of the cholesterol precursor desmosterol were increased in cerebellum $(\mathrm{t}(8)=5.77$; $\mathrm{P}<0.001$ ) of the APPSLxPS1mut mice in comparison with wild-types. The level of the cholesterol precursor lathosterol was slightly reduced in the cortex $(\mathrm{t}(8)=4.13$; $\mathrm{P}<0.01$ ) (Fig. 3A). Total levels of cholesterol in brain remained unaffected (Fig. 3C). The levels of 27-OHcholesterol were significantly increased in the cerebellum of the APPSLxPS1mut mice $(\mathrm{t}(8)=3.63 ; \mathrm{P}<0.01)$, while levels of the brain-specific cholesterol metabolite 24(S)-OHcholesterol were comparable (Fig. 3E). At the age of 21 months the levels of desmosterol, were further (2 to 3-fold) increased in the hippocampus $(\mathrm{t}(12)=3.84 ; \mathrm{P}<0.01)$ and in cerebellum $(\mathrm{t}(12)=3.93 ; \mathrm{P}<0.01)$, as well as in the cortex $(\mathrm{t}(11)=2.95 ; \mathrm{P}<0.05)$ of the APPSLxPS1mut mice in comparison to the wild-type mice (Fig. 3B). 
9 MONTHS

21 MONTHS — wild type controls

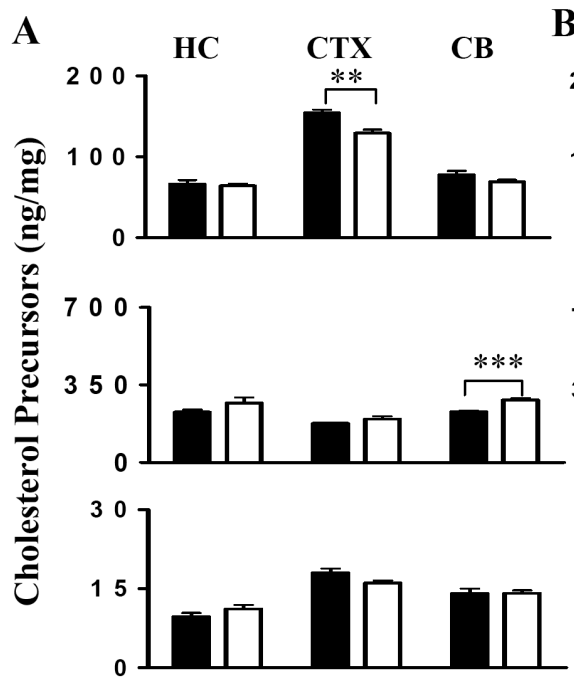

B HC CTX $\quad$ CB $\square$ APPSLxPS1mut
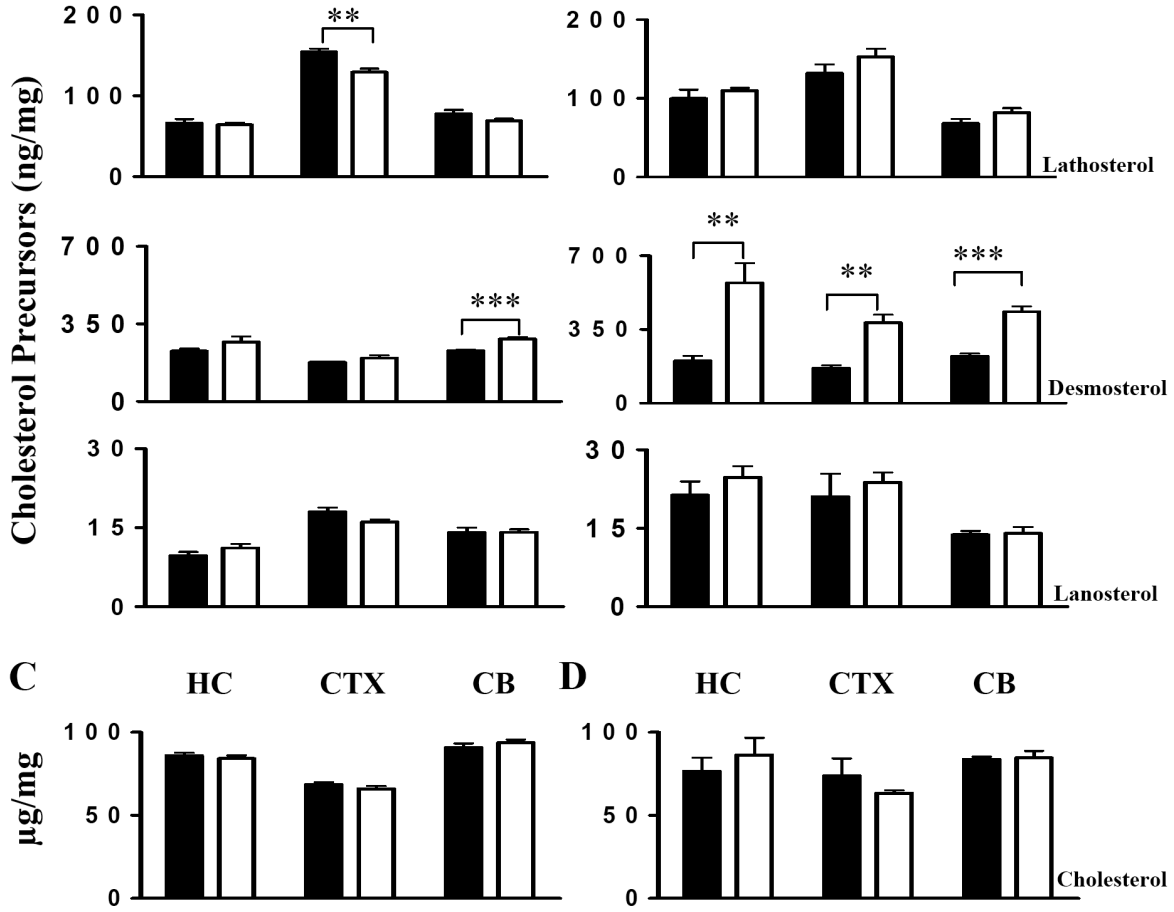

D $\quad \mathrm{HC} \quad \mathrm{CTX} \quad \mathrm{CB}$
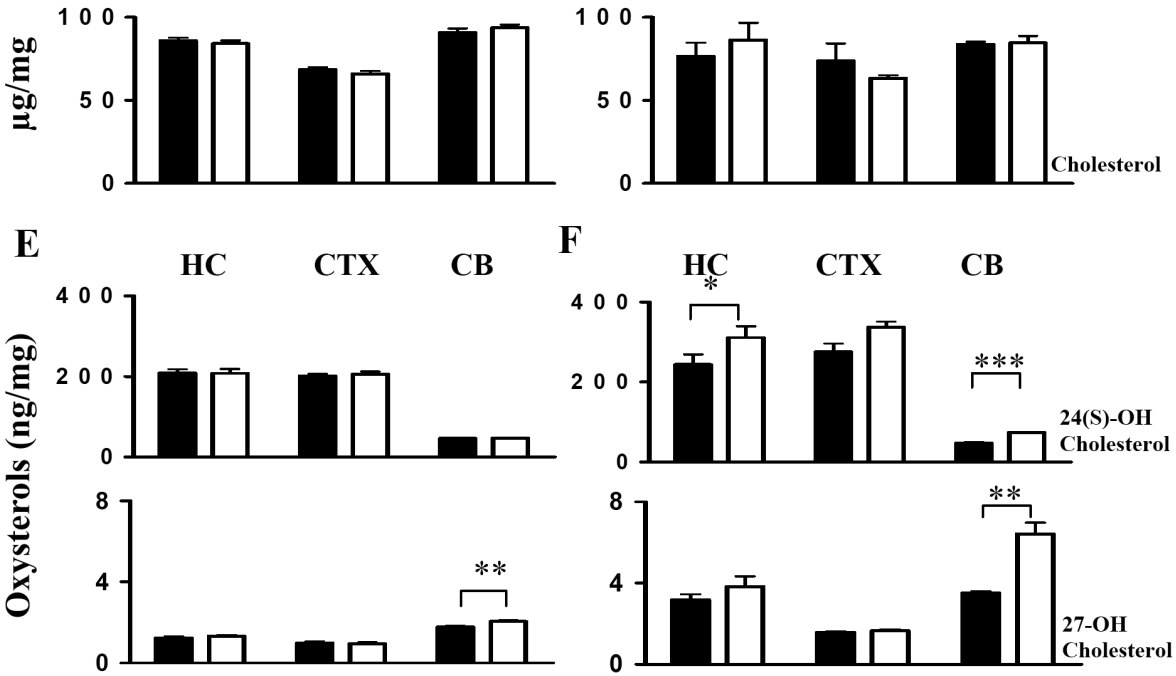

Fig. 3. Sterol levels in brain of APPSLXPS1mut and wild-type mice.

Cholesterol precursor levels (lathosterol, desmosterol and lanosterol) (A \& B), cholesterol (C \& D) and the cholesterol metabolites (24(S)-OHcholesterol and 27-OHcholesterol) (E \& F) in specific brain regions (cerebellum (CB), hippocampus (HC), and cortex (CTX)) of 9 months and 21 months old APPSLXPS1mut $(\mathrm{n}(\mathrm{t}=9$ months $)=5 ; \mathrm{n}(\mathrm{t}=21$ months $)=6)$ and wild-type mice $(\mathrm{n}(\mathrm{t}$ $=9$ months $)=5 ; \mathrm{n}(\mathrm{t}=21$ months $)=7)$. Values are displayed as mean + SEM. $(* \mathrm{P}<0.05, * * \mathrm{P}<0.01$, and $* * * \mathrm{P}<0.001)$. 
Furthermore, at this age, the levels of $24(\mathrm{~S})-\mathrm{OHcholesterol}(\mathrm{t}(12)=3.97 ; \mathrm{P}<0.01)$ were increased significantly in hippocampus and cerebellum and 27-OHcholesterol levels were significantly increased in cerebellum $(t(12)=5.92 ; \mathrm{P}<0.001) \quad$ (Fig. $3 \mathrm{~F}$ ). Despite interregional differences, no differences were detectable in total cholesterol levels within either of the brain regions examined (Fig. 3D).

There were no obvious differences in serum sterol profiles of the APPSLXPS1mut and wild-type mice at both ages (Fig. 4), with the exception of serum total cholesterol levels at 9 months which were slightly lower in the APPSLXPS1mut mice in comparison with the wild type mice $(\mathrm{t}(8)=2.33$; $\mathrm{P}<0.05)$. Livers of the APPSLXPS1mut mice of 21 months displayed significantly increased levels of lathosterol $(10.8 \pm 2.2$ vs. $5.5 \pm 0.3, \mathrm{t}(12)=2,75 ; \mathrm{P}<0.05)$ and decreased levels of 27 OHcholesterol $(1.3 \pm 0.2$ vs. $2.2 \pm 0.2, \mathrm{t}(12)=2.85 ; \mathrm{P}<0.05)$ (data not shown). There were no significant differences in liver sterols at the age of 9 months.

9 MONTHS

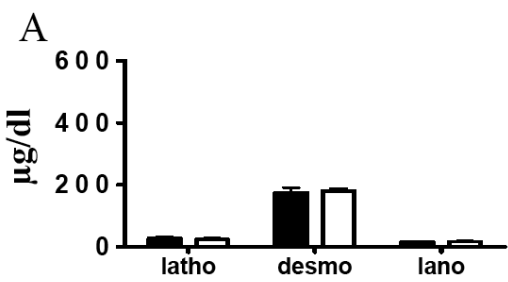

C

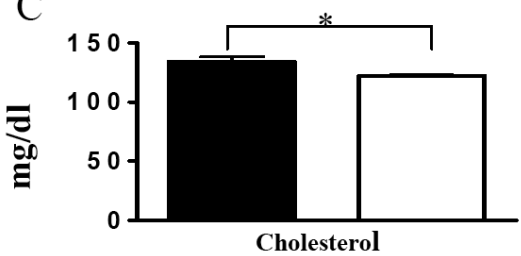

E

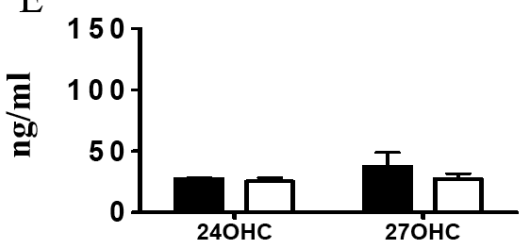

21 MONTHS

B

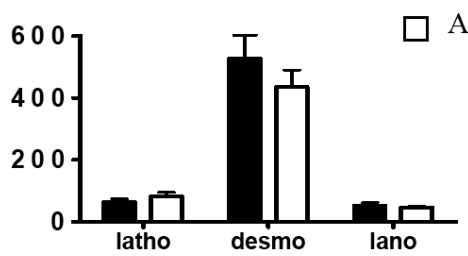

$\mathrm{D}$

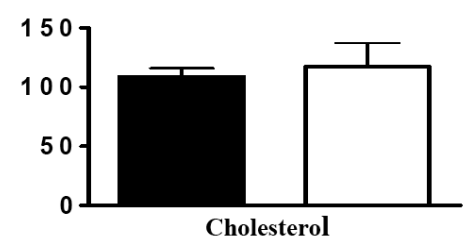

F

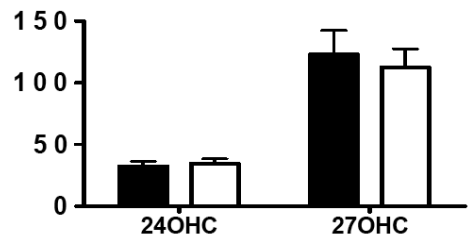

Fig. 4. Serum sterol levels in APPSLxPS1mut and wild-type mice.

Serum cholesterol precursor levels (lathosterol, desmosterol and lanosterol) (A \& B), cholesterol (C \& D) and the major cholesterol metabolites (24(S)-OHcholesterol and 27-OHcholesterol) (E \& F) are shown for APPSLxPS1mut ( $\mathrm{n}=5$ and 6 for 9 and 21 month-old mice, respectively) and wild-type mice ( $\mathrm{n}=4$ and 7 for 9 and 21 month-old mice, respectively). Values are displayed as mean + SEM. 
To find out if the increased levels of desmosterol in the brains of the 21 month old APPSLXPS1mut mice are due to increased synthesis or decreased conversion into cholesterol we quantified the mRNA levels of the selective AD indicator gene (Seladin-1/Dhcr24) and of Cyp51 (Fig. 5). Dhcr24 is an oxidoreductase which catalyzes the reduction of the delta- 24 double bond of sterol intermediates, e.g., lanosterol and desmosterol, hereby generating respectively 24,25-dihydro-lanosterol and cholesterol (Fig. 1). In line with the increased desmosterol levels, Dhcr24 was significantly down-regulated in the cortex $(\mathrm{P}<0.05$; MWU-test $)$ and cerebellum $(\mathrm{P}<0.01$; MWU-test $)$ of the 21-month old APPSLxPS1mut mice versus wild-type mice. Cyp51 is the gene encoding a cytochrome-P450 enzyme (lanosterol 14 alphademethylase) that indirectly converts lanosterol into cholesterol and 24,25-dihydrolanosterol into lathosterol (Fig. 1). There were no differences in Cyp51 mRNA levels at the age of 21 months. However, at 9 months, both Cyp51 ( $<<0.05$; MWUtest) and Dhcr24 ( $<<0.05$; MWU-test) mRNA levels were significantly increased in the hippocampus of APPSLxPS1mut mice. The mRNA levels of the rate-limiting enzyme in the cholesterol biosynthesis, 3-hydroxy-3-methylglutaryl-CoA reductase (Hmgcr), remained unaffected (Fig. 5). Sterol 24-hydroxylase mRNA levels (Cyp46a1) were increased in the cortex and hippocampus $(\mathrm{P}<0.05$; MWU-test $)$ and sterol 27-hydroxylase (Cyp27a1) levels in the cerebellum of APPSLxPS1mut mice at 9 months. Expression levels of Cyp46a1, Cyp27a1 and Hmgcr generally decreased with age (Fig. 5).

Because desmosterol as well as 24(S)-OHcholesterol are activators of the LXR pathway [33, 34], we examined the expression of a number of LXR-target genes; Abca1, Abcg1, Apoe, Apoc1 and Srebp1c. At the age of 9 months Abca1 and Apoe mRNA levels were significantly upregulated in the hippocampus $(\mathrm{P}<0.05$; MWUtest), and interestingly at the age of 21 months Abca1 mRNA was upregulated in the hippocampus $(\mathrm{P}<0.05$; MWU-test) and in the cortex $(\mathrm{P}<0.01$; MWU-test $)$ (Fig. 6). Apoc1, which we identified as a powerfully LXR-responsive gene within the brain (chapter III) and related to learning and memory functions [35], was upregulated in all regions examined at 21 months, but not at 9 months. Apoe, Abcg1 and Srebp1c were upregulated in the hippocampus at the age of 21 months as well (Fig. 6). 
A

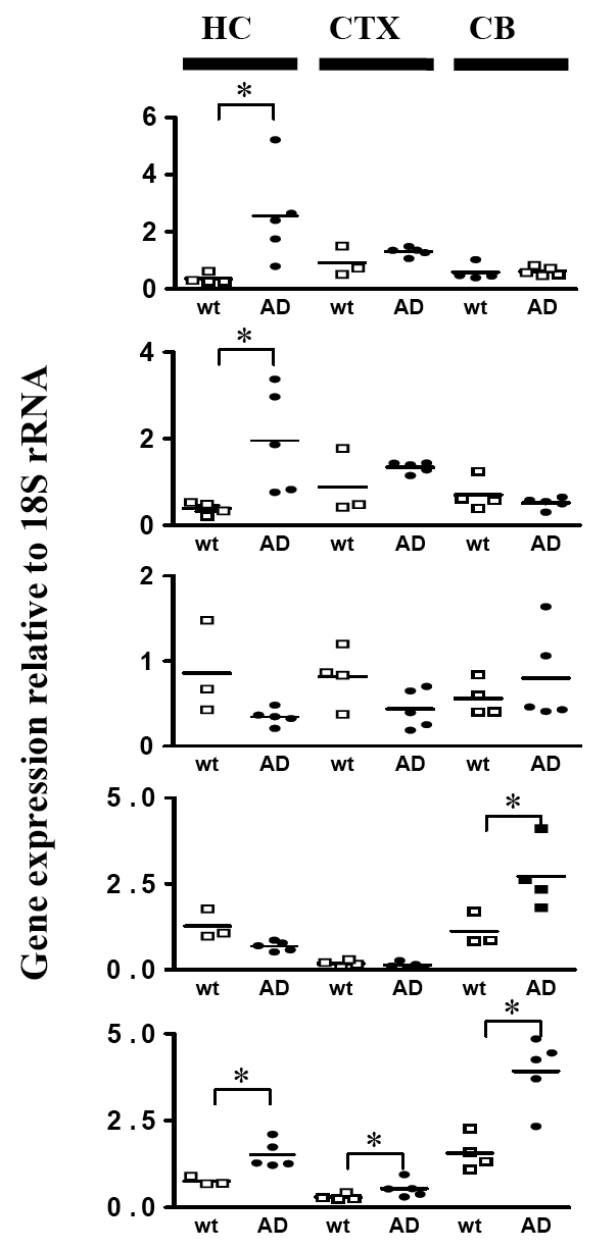

B 21 MONTHS
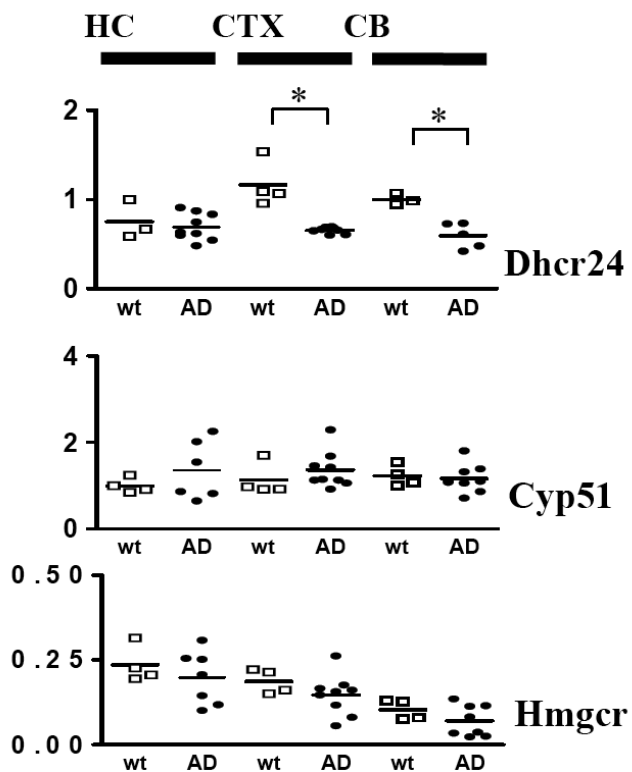

Fig.5. mRNA expression levels of genes involved in brain cholesterol homeostasis.

mRNA expression levels after Q-RT-PCR amplification of cholesterol metabolism regulating genes (Dhcr24, Cyp51, Hmgcr, Cyp27A1 and Cyp46a1), normalized against 18-S-rRNA expression in hippocampus (HC), cortex (CTX) and cerebellum (CB) of 9 months old and 21 months old APPSLxPS1mut $(\mathrm{n}(\mathrm{t}=9$ months $)=5 ; \mathrm{n}(\mathrm{t}=21$ months $)=8)$ and wild-type mice $(\mathrm{n}(\mathrm{t}=$ 9 months $)=4 ; \mathrm{n}(\mathrm{t}=21$ months $)=4)$. Values are displayed as means $+/-$ SEM. Statistics were performed using the MWU-test. $\left(* \mathrm{P}<0.05,{ }^{*} \mathrm{P}<0.01,{ }^{*} \mathrm{P}<0.01\right)$ 
A

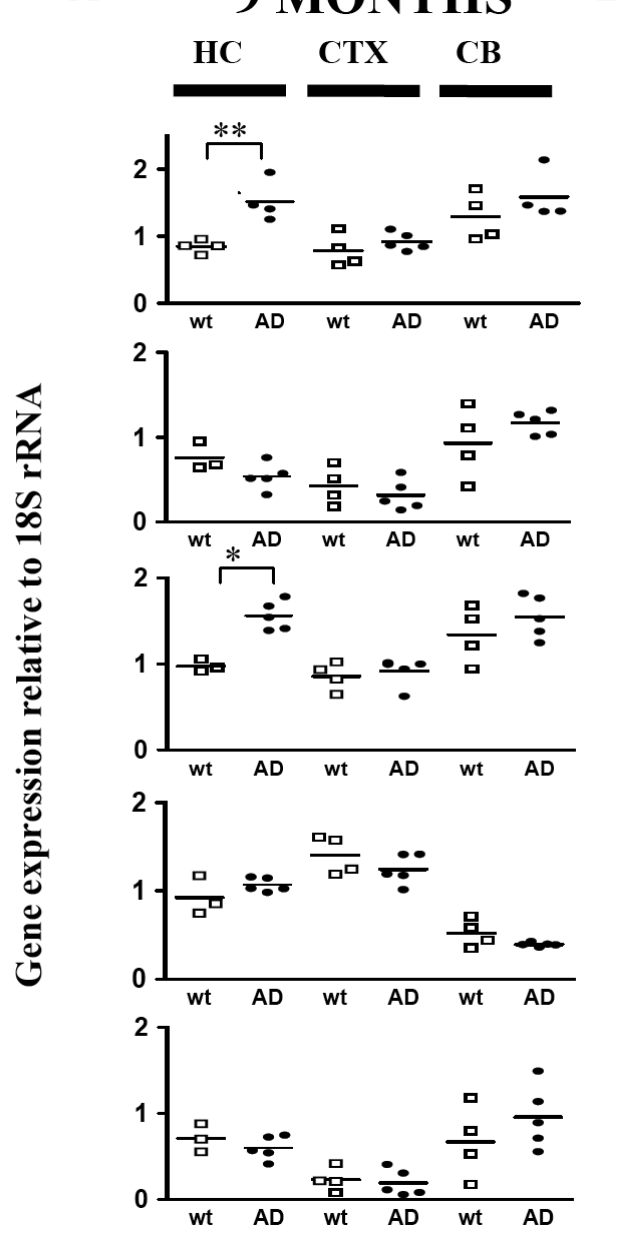

21 MONTHS

HC CTX CB

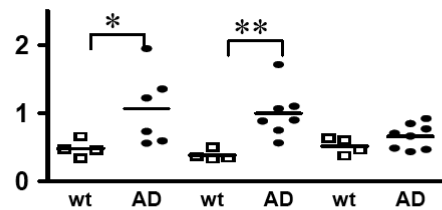

Abca1

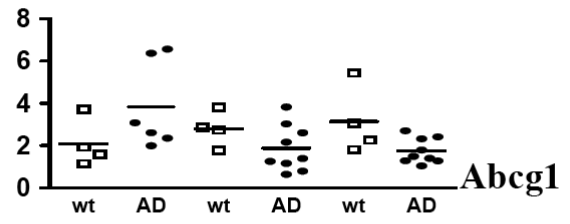

ㅁ
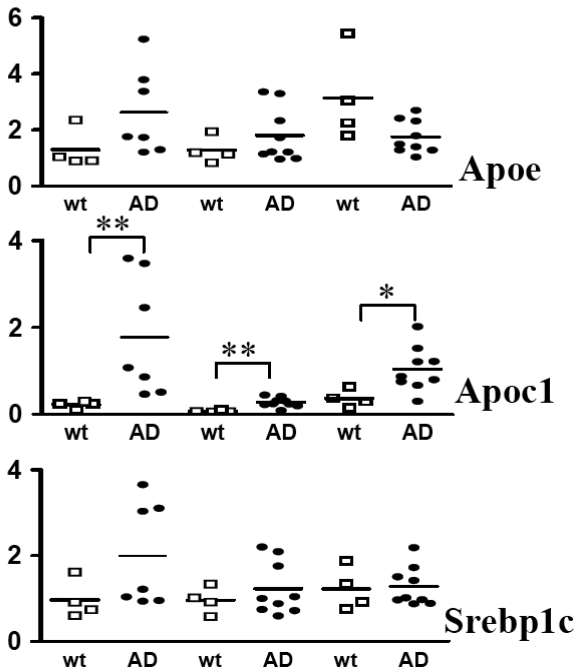

Fig. 6. $m R N A$ expression levels of $L X R$ responsive genes

mRNA expression levels after Q-RT-PCR amplification of LXR responsive genes (Abca1, Abcg1, Apoe Apoc1 and Sreb1c), normalized against 18-S-rRNA expression in hippocampus (HC), cortex (CTX) and cerebellum (CB) of 9 months old and 21 months old APPSLxPS1mut $(\mathrm{n}(\mathrm{t}=9$ months $)=5 ; \mathrm{n}(\mathrm{t}=21$ months $)=8)$ and wild-types $(\mathrm{n}(\mathrm{t}=9$ months $)=4 ; \mathrm{n}(\mathrm{t}=21$ months $)=$ 4). Values are displayed as means $+/$ - SEM. Statistics were performed using the MWU-test. $\left({ }^{*} \mathrm{P}<0.05, * * \mathrm{P}<0.01, * * \mathrm{P}<0.01\right)$ 


\section{Discussion}

Our data strongly suggest that brain cholesterol metabolism is altered as a consequence of the ongoing neuropathology induced by the expression of the APPSL and/or PS1M146L mutants in this murine AD model. These mutations lead amongst others to amyloid deposition, predominantly in the cortex and to a lesser extent in the hippocampus, starting from the age of 6-9 months with a steep increase from the age of 18 months [25].

APPSLXPS1mut mice display age-related alterations in their brain sterol profiles and in the expression of cholesterol metabolism-related genes in comparison with wild-type mice. Brain levels of cholesterol precursors and metabolites were found to be increased in particular in the 21-month old APPSLxPS1mut mice, in parallel with a steep increase in amyloid deposition [25]. Our data suggest an overall enhanced cholesterol turnover in the brain of the APPSLXPS1mut mice, in particular in the hippocampus. Already at the age of 9 months the mRNA levels of Dhcr24 and Cyp51 as well as of Abca1 and Apoe were increased in hippocampus of APPSLXPS1mut mice. However, at that age there was only a limited change in the levels of cholesterol precursors and metabolites detectable. At the age of 21 months, when the brains contained numerous amyloid depositions, desmosterol levels were sturdily increased, suggesting an enhanced overall cholesterol synthesis. The increased levels of 24(S)-OHcholesterol at this age suggest an upregulated conversion and removal of cholesterol from the brain. At the age of 21 months the increase in Cyp51 gene expression was not significantly different between the APPSLXPS1mut and wild-type mice anymore, while Dhcr24 levels even were significantly decreased in cortex and cerebellum of the first. How the overall enhanced cholesterol turnover relates to the pathology remains to be determined since the cortex of aged APPSLxPS1mut mice does display numerous $A \beta$ deposits [25], while the cerebellum remains free of $A \beta$ deposits.

The expression of APPSL and PS1M146L mutants may either directly induce alterations in cholesterol metabolism or indirectly via the ongoing neuropathogenesis, other than the deposition of $A \beta$. At present we can not distinguish which of the mutants underlie the alterations in cholesterol metabolism. However, determination Abca1, Abcg1, Apoe and Srebp1c mRNA levels in the hippocampus of 21 month old mice expressing the APPSL mutant exclusively $(n=2)$ indicated that these are very similar to the wild-type mice (data not shown). This suggests that either the PS1M146L mutation alone or in combination with the 
APPSL mutation is responsible for the alterations in the brain cholesterol metabolism.

In line with our data, overexpression of APP695 in neuroblastoma cells was found to upregulate cholesterol synthesis [36] and $A \beta$ promoted lipid release from neuronal membranes [37]. Others, however, reported a reduction in membrane cholesterol in brains of 9 months, but not of 3 months old APP695swePS1M146L mice [38]. Moreover, an inhibiting effect of $\mathrm{A} \beta 40$ on the activity of Hydroxymethylglutaryl-CoA reductase was reported [20]. Eighteen month old APP23 mice, expressing human APP751 with the Swedish mutation, displayed no alterations in brain levels of cholesterol, its precursors or metabolites [28]. In wildtype mice and also in the APP23 mice desmosterol levels were found to decrease with aging [28]. These finding would be in line with a modulatory role of the PS1M146L mutant. Synthesis of cholesterol via desmosterol along the "Bloch pathway" is the predominant pathway during development, while cholesterol synthesis via 24,25 dihydro-lanosterol and lathosterol along the "Kandutsch-Russell pathway" later in life becomes the predominant pathway (Fig. 1). As a result desmosterol levels decrease dramatically with age in humans [39]. However, we found an increase with aging of the desmosterol levels in the APPSLxPS1mut mice leading to a 2 to 3 -fold increase in desmosterol levels in 21 month-old APPSLxPS1mut mice in comparison with the wild-type mice.

The increased levels of desmosterol in the brains of the APPSLxPS1mut mice may be caused, at least partly, by a reduced activity of Dhcr24 as indicated by its reduced mRNA levels. However, the observation that Dhcr24 mRNA is upregulated at 9 months, but down regulated at 21 months, suggests that reduced Dhcr24 levels are secondary to AD pathology. Moreover, it remains to be explained why Dhcr24 mRNA levels were reduced in cortex and cerebellum, while in cortex numerous amyloid deposits occur, but none in cerebellum. Increased desmosterol levels are in line with an enhanced "Bloch pathway" activation which is presumed to be particularly used by astrocytes, indicating an astrocyte mediated cholesterol upregulation is involved [40].

Reduced Dhcr24 mRNA levels have been observed in post-mortem AD brains, whereas increasing its expression was found to be protective against $A \beta$-induced toxicity in vitro [41]. Dhcr24 has been related to lipid raft formation and decreased Seladin-1 levels increased the co-localisation of APP and $\beta$-secretase, resulting in an increased $\mathrm{AD}$ pathology [41]. It has therefore been suggested that pharmacological enhancement of the Seladin-1-dependent cholesterol synthesis may be an effective A $\beta$-lowering approach for treatment of AD. Since Dhcr24 was 
recently identified as an LXR-target gene, activators of the LXR-pathway may be suitable candidates [42].

In line with the increased levels of 24(S)-OHcholesterol in the brains of APPSLXPS1mut mice, AD patients have been reported to display elevated levels of 24(S)-OHcholesterol in their cerebrospinal fluid [43, 44]. There was no detectable difference in total levels of cholesterol or 24(S)-OHcholesterol at 9 months in the hippocampus and cortex of APPSLxPS1mut mice despite a significant increase in Cyp46a1 mRNA expression.

Although 27-OHcholesterol is mainly produced outside the brain, the cerebellar increase in 27-OHcholesterol in APPSLxPS1mut mice was in parallel with an increased Cyp27a1 mRNA expression in cerebellum at 9 months. Higher levels of 27-OH cholesterol have been described to be protective in AD [45]. This may be related to the observation that no plaques develop within the cerebellum of the APPSLxPS1mut mice.

Increased endogenous cholesterol synthesis and increased levels of desmosterol could be affected by an altered function of the $\gamma$-secretase complex due to the PS1M146L. Loss of function of the $\gamma$-secretase complex has been reported to lead to a decreased lipoprotein uptake [46]. However, it is still unclear how the M146L mutation in the PS1 affects the internalisation of lipoproteins. Our data suggest that overexpression of PS1M146L may lead to increased cholesterol synthesis and turnover.

In conclusion, our data provide evidence that alterations brain cholesterol metabolism can be a consequence of expression of APPSL and/or of PS1M146L directly or via the neuropathology that develops in the APPSLxPS1mut mouse model.

\section{Acknowledgements}

The project was financially supported by Marie Curie Early Stage Training Fellowship: contract number MEST-CT-2005-02058. All experimental procedures concerning the used mice were approved by the local ethical committee of the Maastricht University for animal experiments according to governmental guidelines. There are no conflicts of interest to be disclosed. 


\section{References}

1. Tanzi, R.E., Molecular genetics of Alzheimer's disease and the amyloid beta peptide precursor gene. Ann Med, 1989. 21(2): p. 91-4.

2. Huttunen, H.J., et al., Novel N-terminal Cleavage of APP Precludes Abeta Generation in ACATDefective AC29 Cells. J Mol Neurosci, 2009. 37(1): p. 6-15.

3. Osenkowski, P., et al., Direct and potent regulation of gamma-secretase by its lipid microenvironment. J Biol Chem, 2008. 283(33): p. 22529-40.

4. Xiong, H., et al., Cholesterol retention in Alzheimer's brain is responsible for high beta- and gamma-secretase activities and Abeta production. Neurobiol Dis, 2008. 29(3): p. 422-37.

5. Lund, E.G., J.M. Guileyardo, and D.W. Russell, cDNA cloning of cholesterol 24-hydroxylase, a mediator of cholesterol homeostasis in the brain. Proc Natl Acad Sci U S A, 1999. 96(13): p. 7238-43.

6. Lund, E.G., et al., Knockout of the cholesterol 24-hydroxylase gene in mice reveals a brainspecific mechanism of cholesterol turnover. J Biol Chem, 2003. 278(25): p. 22980-8.

7. Rebeck, G.W., Cholesterol efflux as a critical component of Alzheimer's disease pathogenesis. J Mol Neurosci, 2004. 23(3): p. 219-24.

8. Shafaati, M., et al., Levels of ApoE in cerebrospinal fluid are correlated with Tau and 24Shydroxycholesterol in patients with cognitive disorders. Neurosci Lett, 2007. 425(2): p. 78-82.

9. Antoine, J.C., et al., Pathology of roots, spinal cord and brainstem in syringomyelia-like syndrome of Tangier disease. J Neurol Sci, 1991. 106(2): p. 179-85.

10. Tint, G.S., et al., Markedly increased tissue concentrations of 7-dehydrocholesterol combined with low levels of cholesterol are characteristic of the Smith-Lemli-Opitz syndrome. J Lipid Res, 1995. 36(1): p. 89-95.

11. Marx, J., Alzheimer's disease. Bad for the heart, bad for the mind? Science, 2001. 294(5542): p. 508-9.

12. Puglielli, L., et al., Role of acyl-coenzyme a: cholesterol acyltransferase activity in the processing of the amyloid precursor protein. J Mol Neurosci, 2004. 24(1): p. 93-6.

13. Repa, J.J., et al., Liver $X$ receptor activation enhances cholesterol loss from the brain, decreases neuroinflammation, and increases survival of the NPC1 mouse. J Neurosci, 2007. 27(52): p. 14470-80.

14. Travis, J., New piece in Alzheimer's puzzle. Science, 1993. 261(5123): p. 828-9.

15. Crisby, M., L.A. Carlson, and B. Winblad, Statins in the prevention and treatment of Alzheimer disease. Alzheimer Dis Assoc Disord, 2002. 16(3): p. 131-6.

16. Haag, M.D., et al., Statins are associated with a reduced risk of Alzheimer disease regardless of lipophilicity. The Rotterdam Study. J Neurol Neurosurg Psychiatry, 2009. 80(1): p. 13-7.

17. Rosenberg, P.B., et al., Effects of cardiovascular medications on rate of functional decline in Alzheimer disease. Am J Geriatr Psychiatry, 2008. 16(11): p. 883-92.

18. Shie, F.S., et al., Diet-induced hypercholesterolemia enhances brain $A$ beta accumulation in transgenic mice. Neuroreport, 2002. 13(4): p. 455-9.

19. Reiss, A.B., Cholesterol and apolipoprotein E in Alzheimer's disease. Am J Alzheimers Dis Other Demen, 2005. 20(2): p. 91-6.

20. Grimm, M.O., et al., Regulation of cholesterol and sphingomyelin metabolism by amyloid-beta and presenilin. Nat Cell Biol, 2005. 7(11): p. 1118-23.

21. Grimm, M.O., et al., Altered membrane fluidity and lipid raft composition in presenilin-deficient cells. Acta Neurol Scand Suppl, 2006. 185: p. 27-32.

22. Pedrini, S., et al., Modulation of statin-activated shedding of Alzheimer APP ectodomain by ROCK. PLoS Med, 2005. 2(1): p. e18.

23. Shobab, L.A., G.Y. Hsiung, and H.H. Feldman, Cholesterol in Alzheimer's disease. Lancet Neurol, 2005. 4(12): p. 841-52.

24. Hsiao, K., et al., Correlative memory deficits, Abeta elevation, and amyloid plaques in transgenic mice. Science, 1996. 274(5284): p. 99-102. 
25. Kohler, C., et al., Alzheimer's disease-like neuropathology of gene-targeted APP-SLxPS1mut mice expressing the amyloid precursor protein at endogenous levels. Neurobiol Dis, 2005. 20(2): p. 528-40.

26. Duff, K., et al., Increased amyloid-beta42(43) in brains of mice expressing mutant presenilin 1. Nature, 1996. 383(6602): p. 710-3.

27. Jansen, P.J., et al., Dietary plant sterols accumulate in the brain. Biochim Biophys Acta, 2006.

28. Lutjohann, D., et al., Profile of cholesterol-related sterols in aged amyloid precursor protein transgenic mouse brain. J Lipid Res, 2002. 43(7): p. 1078-85.

29. Heid, C.A., et al., Real time quantitative PCR. Genome Res, 1996. 6(10): p. 986-94.

30. Plosch, T., et al., Increased hepatobiliary and fecal cholesterol excretion upon activation of the liver X receptor is independent of ABCA1. J Biol Chem, 2002. 277(37): p. 33870-7.

31. Dixon, W.J., Analyses of extreme values. Annals of Mathematical Statistics, 1959. 21: p. 488-506.

32. Dixon, W.J., Ratios involving extreme values. Annals of Mathematical Statistics, 1959. 22: p. 6878.

33. Lehmann, J.M., et al., Activation of the nuclear receptor LXR by oxysterols defines a new hormone response pathway. J Biol Chem, 1997. 272(6): p. 3137-40.

34. Yang, C., et al., Sterol intermediates from cholesterol biosynthetic pathway as liver $X$ receptor ligands. J Biol Chem, 2006. 281(38): p. 27816-26.

35. Abildayeva, K., et al., Human apolipoprotein CI expression in mice impairs learning and memory functions. J Lipid Res, 2007.

36. Wirths, O., et al., Altered cholesterol metabolism in APP695-transfected neuroblastoma cells. Brain Res, 2007. 1152: p. 209-14.

37. Gong, J.S., et al., Amyloid beta-protein affects cholesterol metabolism in cultured neurons: implications for pivotal role of cholesterol in the amyloid cascade. J Neurosci Res, 2002. 70(3): p. 438-46.

38. Yao, J.K., et al., Reduced membrane lipids in the cortex of Alzheimer's disease transgenic mice. Neurochem Res, 2009. 34(1): p. 102-8.

39. Fumagalli, R. and R. Paoletti, The identification and significance of desmosterol in the developing human and animal brain. Life Sci, 1963. 5: p. 291-5.

40. Nieweg, K., H. Schaller, and F.W. Pfrieger, Marked differences in cholesterol synthesis between neurons and glial cells from postnatal rats. J Neurochem, 2009. 109(1): p. 125-34.

41. Crameri, A., et al., The role of seladin-1/DHCR24 in cholesterol biosynthesis, APP processing and Abeta generation in vivo. Embo J, 2006. 25(2): p. 432-43.

42. Wang, Y., et al., The selective Alzheimer's disease indicator-1 gene (Seladin-1/DHCR24) is a liver X receptor target gene. Mol Pharmacol, 2008. 74(6): p. 1716-21.

43. Papassotiropoulos, A., et al., 24S-hydroxycholesterol in cerebrospinal fluid is elevated in early stages of dementia. J Psychiatr Res, 2002. 36(1): p. 27-32.

44. Schonknecht, P., et al., Cerebrospinal fluid 24S-hydroxycholesterol is increased in patients with Alzheimer's disease compared to healthy controls. Neurosci Lett, 2002. 324(1): p. 83-5.

45. Scott Kim, W., et al., Impact of 27-hydroxycholesterol on amyloid-beta peptide production and ATP-binding cassette transporter expression in primary human neurons. J Alzheimers Dis, 2009. 16(1): p. 121-31.

46. Tamboli, I.Y., et al., Loss of gamma-secretase function impairs endocytosis of lipoprotein particles and membrane cholesterol homeostasis. J Neurosci, 2008. 28(46): p. 12097-106. 



\section{Chapter III}

\section{Liver X Receptor activation restores memory in aged AD mice without reducing amyloid}




\section{Chapter III}

\section{Liver X Receptor Activation Restores Memory in Aged AD Mice Without Reducing Amyloid}

Tim Vanmierlo ${ }^{\mathrm{a}, \mathrm{b}, \mathrm{g}}$, Kris Rutten $^{\mathrm{a}, \mathrm{g}}$, Jos Dederen ${ }^{\mathrm{c}}$, Vincent W. Bloks ${ }^{\mathrm{d}}$, Leonie C. van Vark -van der Zee ${ }^{\mathrm{f}}$, Folkert Kuipers $^{\mathrm{d}}$, Amanda Kiliaan ${ }^{\mathrm{c}}$, Arjan Blokland ${ }^{\mathrm{e}, \mathrm{g}}$, Eric J.G. Sijbrands $^{\mathrm{f}}$, Harry Steinbusch ${ }^{\mathrm{a}, \mathrm{g}}$, Jos Prickaerts ${ }^{\mathrm{a}, \mathrm{g}}$, Dieter Lütjohann ${ }^{\mathrm{b}, \mathrm{g}}$, Monique Mulder $^{\mathrm{a}, \mathrm{f}}$

a Department of Neuroscience, Maastricht University, Maastricht, The Netherlands; b Institute for Clinical Chemistry and Pharmacology, University of Bonn, Bonn, Germany; c Department of Anatomy and Department of Cognitive Neuroscience, Donders Institute for Brain, Cognition, and Behavior, UMC Nijmegen St Radboud, Nijmegen, Nijmegen, The Netherlands; d Department of Pediatrics, University Medical Center Groningen, Groningen, The Netherlands; e Department of Neuropsychology and Psychopharmacology, Maastricht University, Maastricht, The Netherlands; f Department of Internal Medicine, Division of Pharmacology, Vascular and Metabolic Diseases, Erasmus Medical Center, Rotterdam, The Netherlands; ${ }^{\mathrm{g}}$ EURON (European Graduate School for Neuroscience)

Neurobiology of Aging 2009 [Epub ahead of print] 


\section{Abstract}

Alterations in cerebral cholesterol metabolism are thought to play a role in the progression of Alzheimer's disease (AD). Liver X Receptors (LXRs) are key regulators of cholesterol metabolism. The synthetic LXR activator, T0901317 has been reported to improve memory functions in animal models for $\mathrm{AD}$ and to reduce amyloid- $\beta(\mathrm{A} \beta)$ deposition in the brain. Here we provide evidence that long term administration of T0901317 to aged, 21-month old APPSLxPS1mut mice restores impaired memory. Cerebral cholesterol turnover was enhanced as indicated by the increased levels of brain cholesterol precursors and the up regulation of LXR-target genes Abca1, Abcg1, and Apoe. Unexpectedly, the improved memory functions in the APPSLXPS1mut mice after T0901317-treatment were not accompanied by a decrease in $\mathrm{A} \beta$ plaque load in the cortex or hippocampus DG, CA1 or CA3. T0901317 administration also enhanced cerebral cholesterol turnover in aged C57BL/6NCrl mice, but did not further improve their memory functions.

In conclusion, long-term activation of the LXR-pathway restored memory functions in aged APPSLXPS1mut mice with advanced $A \beta$ deposition. However the beneficial effects of T0901317 on memory in the APPSLxPS1mut mice were independent of the $A \beta$ plaque load in the hippocampus, but were associated with enhanced brain cholesterol turnover.

\section{Introduction}

The molecular mechanisms underlying Alzheimer's disease (AD) are not entirely understood and at present there is no all-embracing effective treatment. Therefore, alternative treatments focusing on prevention or retardation of the progression of $\mathrm{AD}$ pathology are required. Increasing evidence indicates a link between $\mathrm{AD}$ and the brain cholesterol metabolism [1-3]. Disturbances in cholesterol metabolism have been linked to neurological disorders such as Smith-Lemli-Opitz syndrome, Niemann-Pick disease type C, Tangier Disease, Huntington's disease, and recently also to $\mathrm{AD}$ [4-8]. A link between cholesterol and $\mathrm{AD}$ is supported by the fact that APOE4, one of the three common human variants of APOE, namely E2, E3 and E4, is a major genetic risk factor for sporadic $\mathrm{AD}$ [9]. Apolipoprotein E (apoE) is a key player in cholesterol transport in the circulation and is thought to have similar functions within the brain [10]. Moreover, cholesterol-lowering drugs, i.e., statins that are widely used to treat hypercholesterolemia, reduced the risk of $\mathrm{AD}$ although the literature remains controversial $[11,12]$. The brain, composes only $2 \%$ of the total body mass, but contains almost $25 \%$ of an individual's whole body unesterified 
cholesterol. Brain cholesterol originates almost exclusively from in situ neosynthesis [13] because circulating cholesterol is prevented from entering the brain by the blood-brain-barrier [14]. Since cholesterol is synthesized continuously and cannot be degraded within the brain, it is removed from the brain predominantly in the form of the relatively polar brain-specific metabolite, 24(S)hydroxycholesterol (24(S)-OHcholesterol), formed by the enzyme cholesterol 24(S)- hydroxylase (CYP46A1) [15]. The remainder is secreted via an unknown pathway that may involve ABCA1 and apoE $[16,17]$.

Liver X receptors (LXRs) are so called key regulators of cellular cholesterol homeostasis [18]. The LXR family comprises two isoforms, LXR $\alpha$ (NR1H3) and LXR $\beta$ (NR1H2), which play an important role in whole body cholesterol homeostasis, and are abundantly present in the brain [19]. Acute activation of LXRs has been shown to decrease $A \beta$ generation, both in vitro and in vivo [20, 21]. Short term administration of different synthetic LXR-agonists to 20 weeks old $\mathrm{Tg} 2576$ mice, a transgenic model for $\mathrm{AD}$, resulted in improved memory functions $[22,23]$. Moreover, APPSwe/PS1dE9 mice that were deficient for either LXR $\alpha$ or LXR $\beta$ were found to display an increased amyloid plaque load [24]. LXR activation was found to attenuate the inflammatory response of microglia to fibrillar $\mathrm{A} \beta$ in vitro [24]. In addition, LXR activation via oral administration of T0901317 has been reported to enhance the overall cholesterol turnover in the body including that in the brain $[6,25]$. This may lead to a reduced deposition of $A \beta$ in several manners. Firstly, via enhancing its secretion from the brain together with cholesterol as a result of an enhanced cholesterol turnover, $A \beta$ might be reduced [26, 27]. Secondly, it may result in increased concentrations of apoE-containing HDL-like particles in the brain interstitial fluid. These may bind $A \beta$ and prevent its deposition, or may direct $\mathrm{A} \beta$ to glial cells for degradation [22].

We hypothesized that long-term LXR-activation by T0901317 leads to improved memory functions as a result of reduced amyloid deposition in the brain induced by an enhanced cholesterol turnover in the brain. For this purpose we used APPLSxPS1mut mice that bear a humanized endogenous APP gene, e.g. part of the murine gene was replaced by the human APP gene carrying the Swedish and the London mutation, and also expressed the PS1 $1_{\mathrm{M} 146 \mathrm{~L}}$ transgene. APPSL is expressed at endogenous levels with a normal central and peripheral expression pattern. This model therefore has been suggested to be more human-like than most other models for AD that in contrast overexpress APP mutants specifically in neurons [28].

Aged APPLSxPS1mut mice and wild type C57BL/6NCrl mice, that were fed either a T0901317 or a vehicle containing diet, were subjected to a number of behavioral tasks, and subsequently brain sterol profiles and amyloid plaque load was determined. 


\section{Materials and Methods}

Overall design

APPSLxPSmut- or C57BL/6NCrl mice received T0901317 or vehicle and were subjected to behavioural tasks. Subsequently, as indicated in the experimental setup in figure 1, the mice were sacrificed and brains were used for analysis of amyloid load, and for determination of sterol profiles and gene expression.

21 months

$\underline{23 \text { months }}$

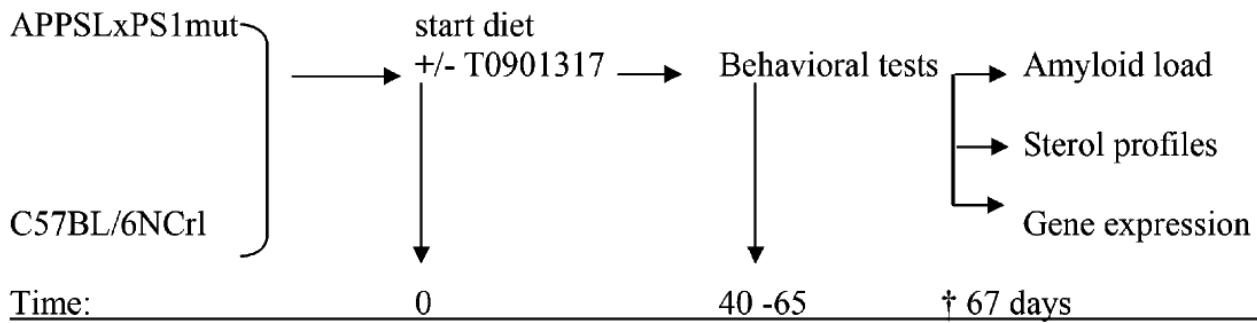

Fig.1. Time scale of the experimental setup.

Animals and Behavior Animals and Diet

Mice with gene-targeted expression of the APP mutant encoding the Swedish/London-FAD mutations were generated as described previously [28] and crossbred with PS1 $1_{\mathrm{m} 1461}$ transgenic mice [29] to generate APPSLxPS1mut mice (mixed Sv129, Black Swiss and C57BL/6NCrl background). Subsequently, mice were backcrossed 6 times with $\mathrm{C} 57 \mathrm{BL} / 6 \mathrm{NCrl}$ mice. The APPSLxPS1mut mice were bred for experimental use in our animal facilities and 1-year old wild type C57BL/6NCrl mice were purchased from Charles River laboratories (L'Arbresle, France) and allowed to age in the same animal facility. At the age of 21 months, male APPSLxPS1mut or C57BL/6NCrl mice were, based on Microsoft Office Excel 2003's random number generator tool, randomly divided into two groups receiving either a T0901317 (Cayman Chemical, Ann Arbor, MI, USA) containing diet (APPSLxPS1mut: $\mathrm{n}=15 ; \mathrm{C} 57 \mathrm{BL} / 6 \mathrm{NCrl}: \mathrm{n}=14$ ) or a control diet (APPSLxPS1mut: $\mathrm{n}=15 ; \mathrm{C} 57 \mathrm{BL} / 6 \mathrm{NCrl}: \mathrm{n}=13$ ).

The most effective T0901317 concentration was first determined in 4 groups $(\mathrm{n}=5)$ of 20 weeks old C57BL/6NCrl mice. Four different concentrations T0901317/ kg 
body weight $(0,10,30$ and $60 \mathrm{mg})$ dissolved in a $5 \%$ ethanol, $0.9 \% \mathrm{NaCl} \mathrm{H}_{2} \mathrm{O}$ solution were administrated by gavages for 6 days. Four hours after the final administration, mice were sacrificed and mRNA was isolated from cortex and hippocampus as described in the section RNA isolation and RT-procedures. Q-RTPCR on ABCA1, a strong T0901317 target gene, was maximally increased at the concentration of $30 \mathrm{mg} / \mathrm{kg}$ body weight (data not shown). Based on the average mouse weight (30g) and on average mouse food consumption (6g/day), experimental food was prepared in a $0.015 \%$ drug in food $(\mathrm{w} / \mathrm{w})$ concentration corresponding to $30 \mathrm{mg} / \mathrm{kg}$ body weight. The diet was prepared by dissolving T0901317 in $0.15 \%$ ethanol as vehicle mixed together with milliq water : crushed (1:1) chow pellets (Ssniff, Soest, Germany). Consequently the mixture was dried at room temperature in order to achieve a $0.015 \%(\mathrm{w} / \mathrm{w})$, drug concentration in food. Chow for the control group was prepared likewise, containing only the ethanol vehicle. One week prior to the beginning of feeding the diets, all animals were single-housed, with ad libitum access to food and water. Mice were subjected to behavioural testing after 6 to 9 weeks of diet in the dark phase of the day-night cycle. Testing was performed with the experimenter being unaware of the individual dietary treatment conditions and the code was broken after mice were sacrificed. Handling procedures were conform previous descriptions [30]. The Kaplan Meier survival analysis for long term T0901317-fed ( $\mathrm{n}=15$ at $\mathrm{t}=0 ; \mathrm{n}=8$ at $\mathrm{t}=67)$ versus the vehicle-fed $(\mathrm{n}=15$ at $\mathrm{t}=0 ; \mathrm{n}=13$ at $\mathrm{t}=67)$ APPSLxPS1mut mice revealed an increased mortality for the T0901317-fed APPSLxPS1mut mice $\left(\chi^{2}(1)=4.03 ; \mathrm{P}<0.05\right)$. None of the $\mathrm{C} 57 \mathrm{BL} / 6 \mathrm{NCrl}$ mice died during the experiment.

\section{Object Recognition Task}

The object recognition task (ORT) was conducted after 6 weeks of T0901317 treatment as described with following specifications [30, 31]. In brief, all animals were habituated to the arena over three days. Mice were allowed to explore the empty arena in trials of $4 \mathrm{~min}$ for two days. On the third day of the habituation, mice were subjected to two trials of 4 minutes in the arena with two similar objects (samples). The two objects were placed symmetrically about $10 \mathrm{~cm}$ away from the wall of the circular arena. After one resting day, the experiment started. During the first trial (T1) the apparatus contained two identical objects (samples). After exploring the samples in the first exploration period of $4 \mathrm{~min}$, the mouse was placed back in its home cage. Subsequently, after a predetermined delay interval $(0.5 \mathrm{~h}, 1 \mathrm{~h}$, $2 \mathrm{~h}$ or $6 \mathrm{~h}$ ), the mouse was returned to the arena for the second trial of $4 \mathrm{~min}$ (T2), but now with two dissimilar objects, a familiar one (the sample) and a new one. The times spent exploring each object during T1 and T2 were recorded manually using a personal computer. Sitting on the object was not considered as exploratory 
behaviour. The discrimination index $\mathrm{d} 2$ in $\mathrm{T} 2(\mathrm{~d} 2=[($ exploration time for the novel object) - (exploration time for the familiar object)] / (total exploration time in T2)) was calculated as measure for object memory.

\section{Object Location Task}

The object location task (OLT), a spatial memory task applied as modified form of the ORT as described above [31], was conducted after 8.5 weeks of T0901317 treatment. Since the animals were already familiar with the arena, no habituation assays were required. At the start of $\mathrm{T} 1$ ( $4 \mathrm{~min})$, two objects were placed symmetrically about $10 \mathrm{~cm}$ away from the wall. After the predetermined delay interval $(0.5 \mathrm{~h}, 1 \mathrm{~h}, 2 \mathrm{~h}$ or $6 \mathrm{~h})$, the mouse was returned to the arena for the second trial of 4 min (T2), but now, one of the two similar objects, was displaced conform with an alternating location scheme. The same objects used in the ORT were applied in the OLT. The times spent exploring each object during T1 and T2 were recorded manually using a personal computer. The discrimination index $\mathrm{d} 2$ in $\mathrm{T} 2 \mathrm{(d} 2=$ [(exploration time for the displaced object) - (exploration time for the familiar object)] / (total exploration time in T2)) was calculated as measure for spatial memory.

\section{Open Field}

The open field test (OF) was conducted after 8 weeks of chronic T0901317 treatment as described previously [32] in a square, clear Plexiglas box $(50 \mathrm{~cm} \times$ $50 \mathrm{~cm} \times 30 \mathrm{~cm}$ ), with an open top and a grey floor. The movements and position of the animals were recorded and registered automatically by a computerized system (Etho Vision ${ }^{\mathrm{TM}}$, Noldus, Wageningen, The Netherlands) in order to determine time in zone (TIZ) and distance moved (DM) over the $10 \mathrm{~min}$ trial.

\section{Analyses}

Sample preparation

The mice were anaesthetized with IP ketamine/xylazine $(100 \mathrm{mg} / \mathrm{kg}$ ketamine and 5 $\mathrm{mg} / \mathrm{kg}$ xylazine), before decapitation. Blood was collected and spun $10 \mathrm{~min}$ at $200 \mathrm{~g}$ to obtain serum, which was snap frozen in liquid nitrogen. Brains were divided into two hemispheres. For APPSLxPS1mut mice, one hemisphere was preserved for cryosection for immuhistochemical (IHC) staining (APPSLxPS1mut: T0901317: $\mathrm{n}=$ 8; vehicle: $\mathrm{n}=8$ ). The other hemisphere was preserved for mRNA Quantitative Real Time Polymerase Chain Reaction (Q-RT-PCR) (APPSLxPS1mut: T0901317: $\mathrm{n}=4$; vehicle: $n=9$ ) analyses or sterol profiling (APPSLxPS1mut: T0901317: $n=4$; vehicle: $\mathrm{n}=9$ ). For the $\mathrm{C} 57 \mathrm{BL} / 6 \mathrm{NCrl}$ mice, one hemisphere was used for sterol 
analysis and the other half for Q-RT-PCR (C57BL/6NCrl : T0901317: $\mathrm{n}=14$; vehicle:n=13).

\section{Aß Quantification}

Brain hemispheres were overnight post fixed in Somogyi fixative (4\% depolymerized paraformaldehyde, $0.2 \%$ picric acid, $50 \% 0.2 \mathrm{M}$ phosphate buffer (pH 7.3)). Next day the hemispheres were transferred to a daily increasing sucrose concentration solution $(10 \%, 20 \%$ and $30 \%$ sucrose in $0.1 \mathrm{M}$ phosphate buffer). Subsequently, brains were $\mathrm{CO}_{2}$ frozen and stored at $-80^{\circ} \mathrm{C}$. Six series of $40-\mu \mathrm{m}$ frontal sections were cut through the brain using a sliding microtome (Microm HM 440, Walldorf, Germany). Immunohistochemistry and plaque quantification were performed using standard free-floating labeling procedures on series 1 and 2 as described before [33]. In brief, after blocking the brain sections against endogenous peroxidase with $0.3 \% \mathrm{H}_{2} \mathrm{O}_{2}$ in phosphate buffer saline, the sections were pretreated with $0.05 \mathrm{M}$ sodium citrate solution at $85^{\circ} \mathrm{C}$ for 20 minutes. Monoclonal mouse anti$\mathrm{A} \beta_{4-10}$ anitbody (1:20.000) was used as primary antibody (T. Hartmann, Homburg, Germany). Biotin labeled donkey-anti-mouse (1:1500) was used as secondary antibody (Jackson Immuno Research, Suffolk, UK). After 90 minutes of incubation the sections were rinsed three times and transferred to a solution containing $\mathrm{ABC}$ elite 1:800 (Vector laboratories, Burlingame) for 90 minutes. Visualization of $A \beta$ was achieved by incubation with 3-3'diaminobenzidine tetra hydrochloride with ammonium nickel sulphate as an intensifier (DAB-Ni solution). All stained sections were mounted on gelatin-coated slides, dried overnight in a stove at $37^{\circ} \mathrm{C}$, dehydrated in alcohol series, cleared with xylol and mounted in Entellan. All sections were stained in one session to minimize differences in staining intensity. The amount of $A \beta$ in the cortex and in the hippocampus, namely in the cornu amonis 1 (CA1), the CA3 and the dentate gyrus (DG), were quantified in five sections (with $200 \mu \mathrm{m}$ distance between the sections). The hippocampus and cortex were quantified at -2.18 up to -2.46 posterior to bregma. The regions were chosen because of their large amyloid load in humans and transgenic mice and their importance in memory [34, 35]. Sections were digitized and quantified, using a Zeis Axioskop microscope equipped with hardware and software of Microbrightfield, (Williston, USA). A $\beta$ load was defined as the percentage of area covered by A $\beta$. All measurements were performed blind by two investigators.

Alternatively, Sodium-Dodecyl-Sulfate (SDS) and formic acid soluble A $\beta$ fractions were detected immunochemically using ELISA kits against human $A \beta 40$ and $A \beta 42$ (Invitrogen, \#KHB3482 and \#KHB3544, Camarillo, CA, USA) after a four phase extraction [36]. 


\section{Sterol profile determination}

Brains were dissected in cerebellum, hippocampus, and cortex. Preceding the analysis, samples were spun in a speed vacuum dryer (12mbar) (Savant AES 1000) for $24 \mathrm{~h}$ to relate individual sterol concentrations to dry weight. The sterols were extracted from the dried tissue by placing them in a $2 \mathrm{ml}$ mixture of chloroformmethanol $(2: 1)$ for $24 \mathrm{~h}$ at $4{ }^{\circ} \mathrm{C}$. Sterol levels were determined by gas chromatograph mass spectrometer as described previously [37,38].

\section{$R N A$ isolation and RT-procedures}

Cerebellum, hippocampus, and cortex were homogenized in $1 \mathrm{ml}$ of Trizol Reagent (In vitrogen, Breda, The Netherlands) preceding the RNA isolation. Total RNA was isolated using the Trizol Reagent method (Invitrogen, Breda, The Netherlands) according to the manufacturer instructions and quantified using Ribogreen (Molecular Probes, Inc., Eugene, OR). cDNA equivalent to 10 ng of total RNA was amplified using the Q-RT-PCR core kit (Eurogentec, Seraing, Belgium) according to the manufacturers protocol. Q-RT-PCR was performed using an Applied Biosystems 7700 sequence detector according to the manufacturer's instructions as described before [39], with modifications [25]. Primer oligomers (Invitrogen, Breda, The Netherlands) and fluorogenic probes, labeled with 6carboxyfluorescein(5'- FAM) and 6-carboxytetramethylrhodamine(3'-TAMRA), were purchased by Eurogentec (Seraing, Belgium). Primer and probe sequences have been deposited at the RTPrimerDB (pubmed: 18948285) and are shown in Table I. All expression data were subsequently normalized for $18 \mathrm{~S}$ rRNA, which was analyzed in a separate run.

Table I: Primer and probe sequences applied in Q-RT-PCR assay

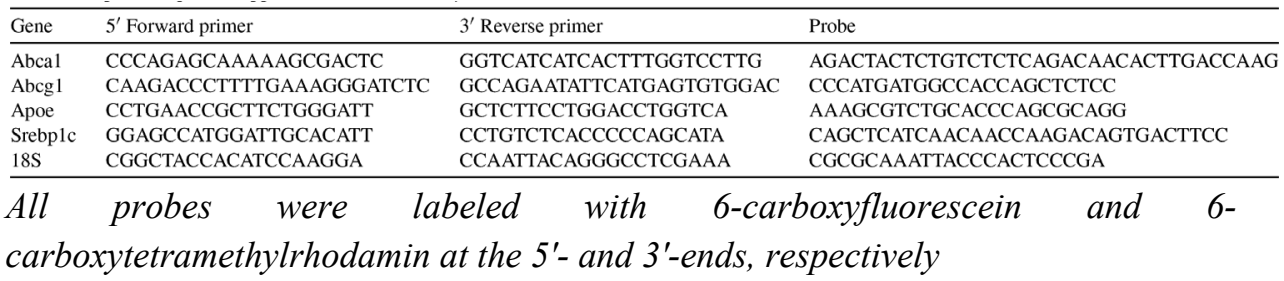

\section{Statistics}

All statistic analyses were performed using GraphPad Prism $4^{\mathrm{TM}}$. The ORT and OLT data were analyzed over time using the 2-way analysis of variances assay (2way ANOVA) with time interval and treatment as independent variables. Animals that did not reach the minimum of 5 seconds exploration in T1 or T2 of the ORT or OLT were excluded from further analysis [31]. d2-Values at the different time intervals were compared between the T0901317 and vehicle fed group using an 
unpaired 2-sided student T-test. In order to distinguish whether $\mathrm{d} 2$ values were different from 0 , a one-sample student T-test was applied. A $\beta$ plaque quantification data were compared using an unpaired two-sided student T-test. When normality could not be proven due to a low power, mRNA and sterol levels were compared using the non parametrical Mann Whitney U (MWU) test. The Kaplan Meier survival analysis was verified on significance using the log rank chi square test. Significance levels were determined on $\mathrm{P}<0.05, \mathrm{P}<0.01$ or $\mathrm{P}<0.001$. In all data sets, extreme values were excluded by means of Dixon's principles of exclusion of extreme values [40, 41].

\section{Results}

Long term T0901317 administration did not induce alterations in appearance or general behavior of the APPSLxPS1mut and the C57BL/6NCrl mice. Body weight of the mice remained stable during the testing period and was not affected by T0901317 treatment (data not shown).

Long term LXR-activation improves memory functions in aged APPSLXPS1mut mice

In line with the neuropathological changes [28], the APPSLxPS1mut mice, displayed a significantly impaired performance in the ORT in comparison with the C57BL/6NCrl mice $(\mathrm{F}(1,88)=15.54 ; \mathrm{P}<0.001$, see Fig. 2A\&B), indicating impaired memory functions. Memory of the APPSLxPS1mut mice declined already after $1 \mathrm{~h}$ $(\mathrm{t}(11)=0.74$; n.s.), while the vehicle treated control $\mathrm{C} 57 \mathrm{BL} / 6 \mathrm{NCrl}$ mice memorized the objects well upto $6 \mathrm{~h}(\mathrm{t}(8)=5.95 ; \mathrm{P}<0.001)$. After T0901317 administration, the APPSLXPS1mut mice displayed a significantly better discrimination between the novel and the familiar object than the vehicle-treated group over the different delay intervals $(\mathrm{F}(1,73)=7.74 ; \mathrm{P}<0.01)$ as shown in Fig. 2A. T0901317 treatment restored memory of APPSLxPS1mut mice until the $2 \mathrm{~h}$ delay interval $(\mathrm{F}(1,55)=2.20 ; \mathrm{n} . \mathrm{s})$, but not at the $6 \mathrm{~h}$ delay interval $(\mathrm{t}(14)=3.14 ; \mathrm{P}<0.01))$. T0901317 treatment did not further improve the performance of the $\mathrm{C} 57 \mathrm{BL} / 6 \mathrm{NCrl}$ mice $(\mathrm{F}(1,84)=0.60$; n.s; see Fig. 2B).

Subsequently, the object location task (OLT) was performed in order to examine the effect of T0901317 administration on spatial memory. While the C57BL/6 NCrl mice memorized the objects well up to $6 \mathrm{~h}(0.5 \mathrm{~h}: \mathrm{t}(12)=7.17 ; \mathrm{P}<0.001,1 \mathrm{~h}$ : $\mathrm{t}(12)=5.66 ; \mathrm{P}<0.001,2 \mathrm{~h}: \mathrm{t}(12)=4.64 ; \mathrm{P}<0.001,6 \mathrm{~h} \mathrm{t}(11)=6.51 ; \mathrm{P}<0.001)$ the vehicletreated APPSLxPS1mut mice could not distinguish the re-located/displaced object from the regularly placed object at any time interval examined $(0.5 \mathrm{~h}: \mathrm{t}(8)=0.20$; n.s., 
1h: $\mathrm{t}(6)=0.40$; n.s., $2 \mathrm{~h}: \mathrm{t}(5)=0.22$; n.s., $6 \mathrm{~h}: \mathrm{t}(6)=0.25$; n.s.). T0901317 treatment significantly improved the performance of the APPSLxPS1mut mice in the OLT $(F(1,50)=15.78 ; P<0.001$, Fig. $2 C)$ upto the $2 \mathrm{~h}$ interval time point $(0.5 \mathrm{~h}: \mathrm{t}(5)=2.88$; $\mathrm{P}<0.05,1 \mathrm{~h}: \mathrm{t}(4)=7.51 ; \mathrm{P}<0.01,2 \mathrm{~h}: \mathrm{t}(5)=3.38 ; \mathrm{P}<0.05)$, but not after $6 \mathrm{~h}$ delay (6h: $\mathrm{t}(5)=0.91$; n.s.). Performance of the C57BL/6NCrl mice in the OLT $(\mathrm{F}(1,99)=0.00$; n.s. Fig. 2D) was not further improved by T0901317 treatment.
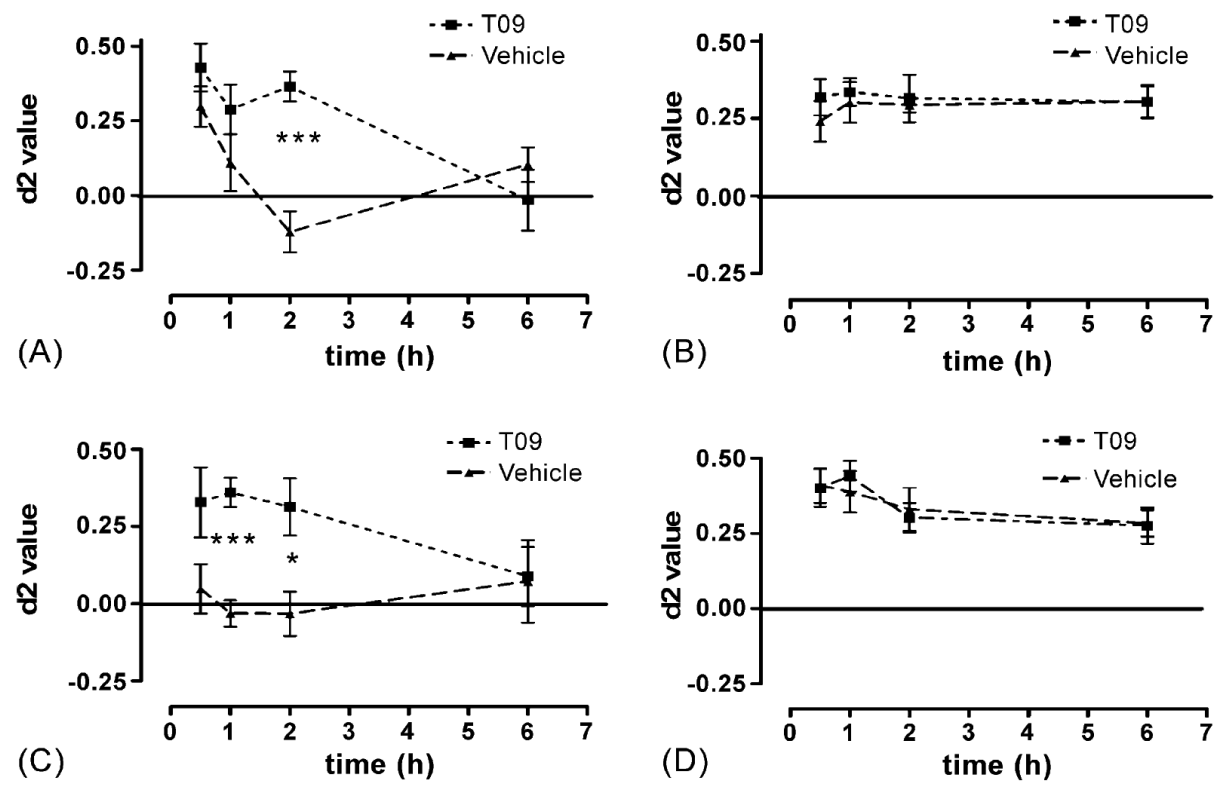

Fig. 2. The effects of chronic T0901317 treatment in APPSLxPS1mut mice and C57BL/6NCrl mice in the object recognition task and object location task.

Object memory in the ORT improves after T0901317 (T09) treatment of APPSLxPS1mut (A), but remains uninfluenced in C57BL/6NCrl (B) mice. T0901317 treatment ameliorated spatial memory in APPSLxPS1mut mice (C), but did not affect performance of C57BL/6 $\mathrm{NCrl}$ mice (D). Total exploration times in trial 1 (T1) and in trial 2 (T2) of ORT and OLT, were comparable between all 4 groups (data not shown). (Significant differences as determined by unpaired 2 -sided T-test, are indicated $*=\mathrm{P}<0.05 ; * * *=\mathrm{P}<0.001)$. Values are displayed as means $+/$ - SEM.

General locomotor activity or anxiety of the APPSLxPS1mut or C57BL/6NCrl mice was not affected by long term T0901317-treatment.

General locomotor behavior and anxiety was examined using the Open Field task (OF) (Table II). T0901317 had no affect on the time spent in the center of the arena or the distance moved by the APPSLxPS1mut mice, nor by the C57BL/6NCrl mice (Table II). Therefore, T0901317 treatment did not detectably affect locomotor activity or anxiety. However, the APPSLXPS1mut mice, spent significantly more time in the center of the arena $(\mathrm{t}(18)=2.23 ; \mathrm{P}<0.05)$ and covered a significantly shorter distance $(\mathrm{t}(24)=3.61 ; \mathrm{P}<0.001)$ than the $\mathrm{C} 57 \mathrm{BL} / 6 \mathrm{NCrl}$ mice. 
Table II: APPSLxPS1 and C57BL/6NCrl, mice in the open field task: Time in zone (TIZ) and Distance Moved (DM)

\begin{tabular}{llcllll}
\hline & T09 & TIZ center $(\mathrm{s})$ & TIZ corner $(\mathrm{s})$ & TIZ walls $(\mathrm{s})$ & DM $(\mathrm{m})$ \\
\hline C57BL/6NCRL & - & $89.5^{\mathrm{a}}(6.55)$ & $186.2(9.21)$ & $324.4(8.32)$ & $38.2(3.11)$ & 12 \\
& + & $107.2(9.28)$ & $194.0(9.90)$ & $298.8(7.81)$ & $34.5(1.38)$ & 14 \\
APPSLxPS1mut & - & $208.8(24.14)$ & $134.9(13.08)$ & $256.3(17.01)$ & $23.6(2.63)$ & 14 \\
& + & $176.8(47.63)$ & $139.4(51.42)$ & $283.3(48.79)$ & $20.5(5.24)$ & 8 \\
\hline
\end{tabular}

a Values are displayed as mean \pm (SEM).

T0901317 treatment alters brain cholesterol homeostasis in APPSLXPS1mut and in C57BL/6NCrl mice.

T0901317 treatment resulted in increased levels of cholesterol precursors (lathosterol, lanosterol and desmosterol) in cerebellum (CB), hippocampus (HC), and cortex (CTX) in APPSLxPS1mut and in C57BL/6NCrl mice (Fig. 3A \& B). Yet, the levels of lathosterol in the HC and lanosterol in the CTX of the APPSLXPS1mut mice and lanosterol and desmosterol in the CTX of the C57BL/6NCrl mice lacked the power to reach significance. Levels of total cholesterol and of the cholesterol metabolites, 24(S)-OHcholesterol and 27OHcholesterol remained unaffected in most brain regions of the APPSLXPS1mut mice (Fig. 3C and E) and C57BL/6NCrl mice (Fig. 3D and F) after T0901317 treatment. Only 24(S)-OHcholesterol and 27-OHcholesterol levels in $\mathrm{CB}$ of the C57BL/6NCrl mice were decreased (Fig. 3F). Serum cholesterol precursor levels significantly increased 1.3- and 1.8-fold, respectively in APPSLXPS1mut and C57BL/6NCrl mice upon T0901317 treatment (data not shown). Livers of the T0901317-treated APPSLxPS1mut and C57BL/6NCrl mice displayed a 1.3-fold decrease in cholesterol and an about 2.5-fold increase in cholesterol metabolites. Plant sterol levels in both liver and serum were about 6-fold decreased upon T0901317 treatment (data not shown). 


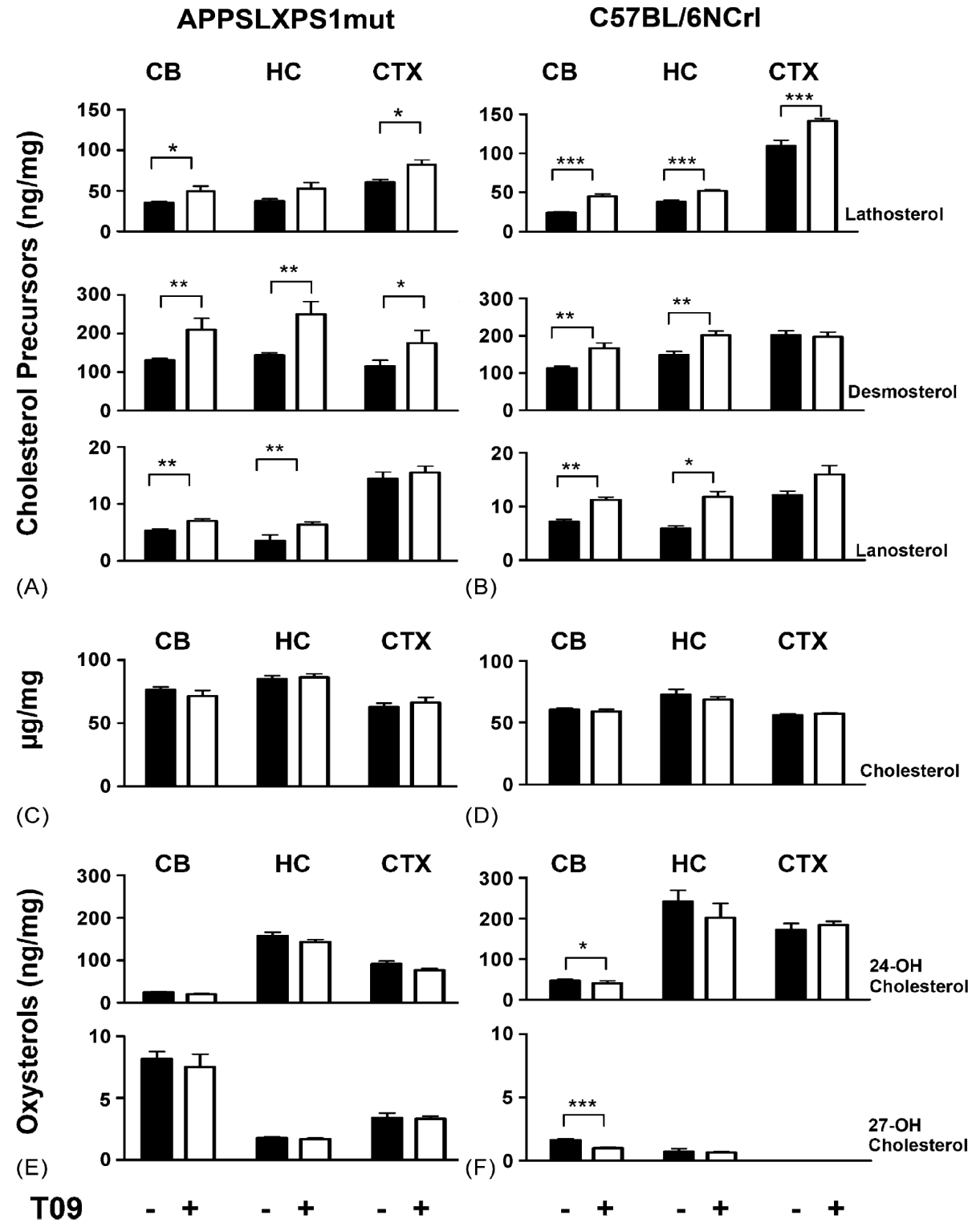

Fig.3. Sterol levels in different brain regions of APPSLxPS1mut and C57BL/6NCrl mice.

Cholesterol precursor levels (lathosterol, desmosterol and lanosterol) (A \& B), cholesterol (C \& D) and the main cholesterol metabolites (24S-OHcholesterol and 27-OHcholesterol) (E \& F) are displayed for specific brain regions (cerebellum (CB), hippocampus (HC), and cortex (CTX)) after 67 days of diet containing T0901317 (T09+) or vehicle (T09-) in aged APPSLxPS1mut $(\mathrm{n}(\mathrm{T} 09-)=8$ and $\mathrm{n}(\mathrm{T} 09+)=4)(\mathrm{A}, \mathrm{C}$, and $\mathrm{E})$ and C57BL/6NCrl mice $(\mathrm{n}(\mathrm{T} 09-)=13$ and $\mathrm{n}(\mathrm{T} 09+)=14)$ $(\mathrm{B}, \mathrm{D}$, and $\mathrm{F})$. Values are displayed as means $+/-\mathrm{SD} .\left({ }^{*} \mathrm{P}<0.05, * * \mathrm{P}<0.01\right.$, and $\left.* * * \mathrm{P}<0.001\right)$ 
Upregulation of LXR target genes in brain of APPSLXPS1mut and C57BL/6NCrl mice after long term T0901317 treatment.

Data presented in Figure 4A show the effect of long term T0901317 treatment on expression of a number of LXR-target genes. In HC and CB of the APPSLXPS1mut mice Abcal and Srebp1c mRNA levels were 2 to 3-fold increased by T0901317. However, no effect was observed in the CTX. Abcg1 and Apoe expression were significantly increased in the $\mathrm{CB}$ and to a lesser extend also in the HC. Basal expression of Abca1, Abcg1, Apoe and Srebp1c in C57BL/6NCrl mice was on average three times lower than in APPSLxPS1mut mice, and was increased by T0901317 in HC, CB and unlike in the APPSLxPS1mut mice also in CTX.

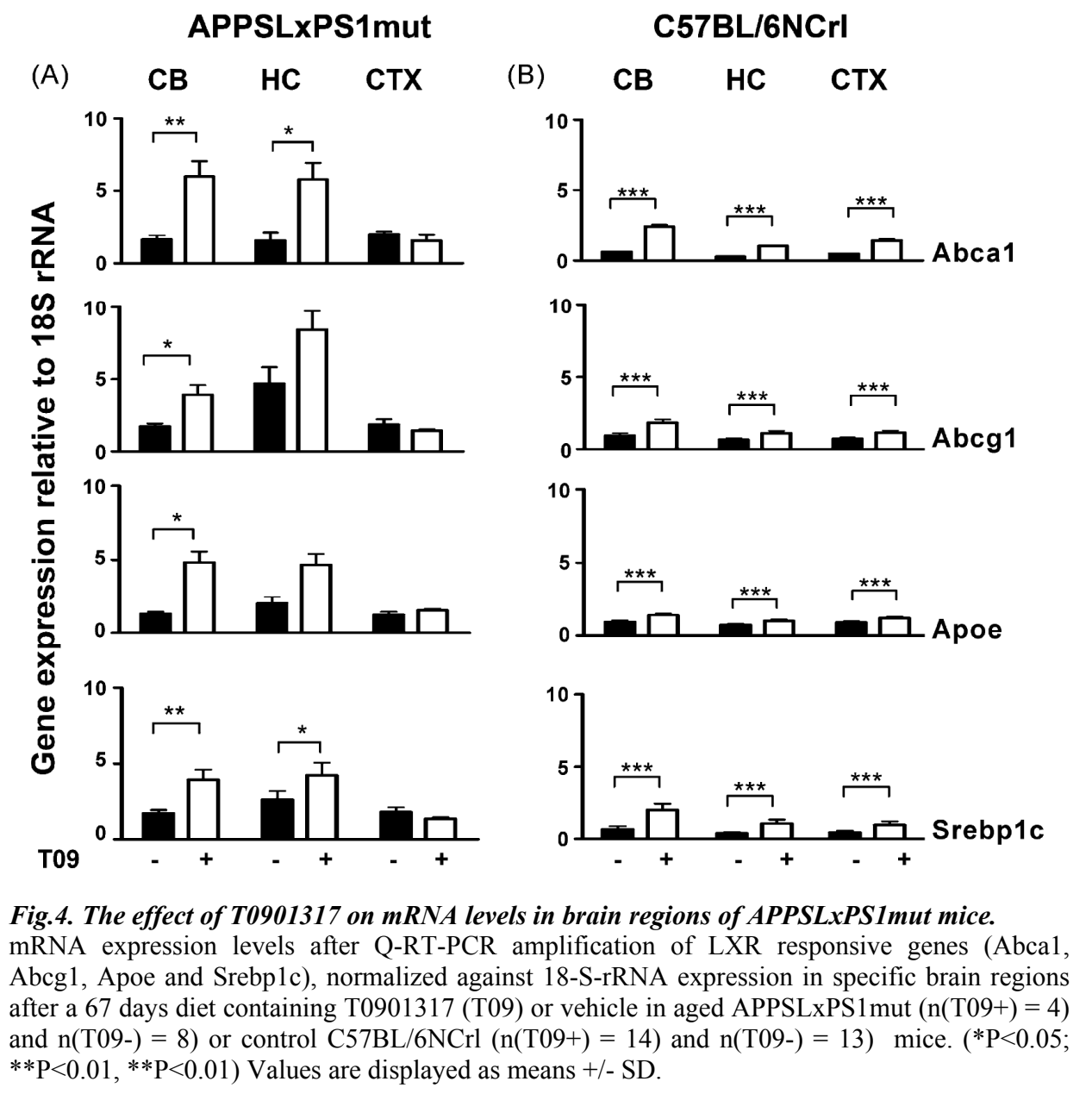


(A)
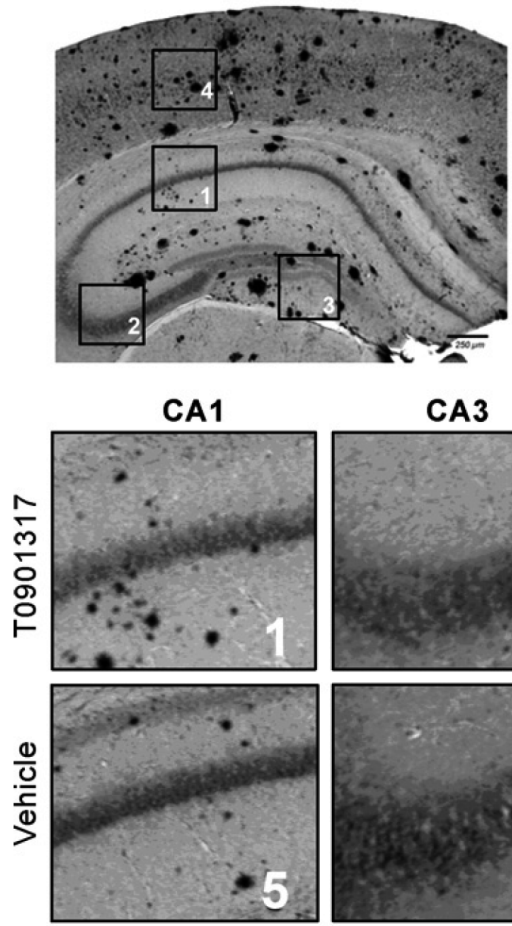

(B)

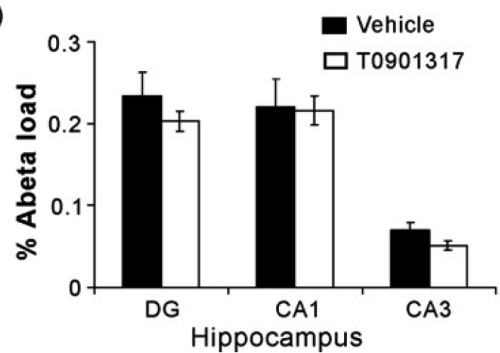

CA3
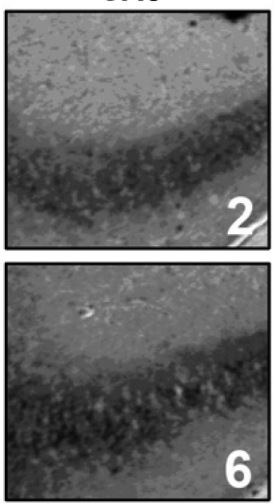

6.

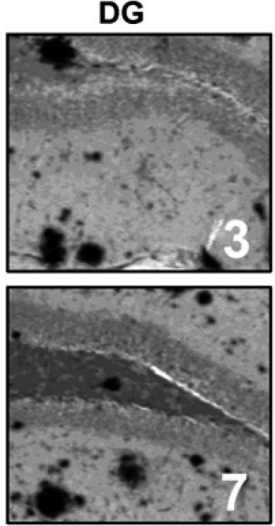

(C)

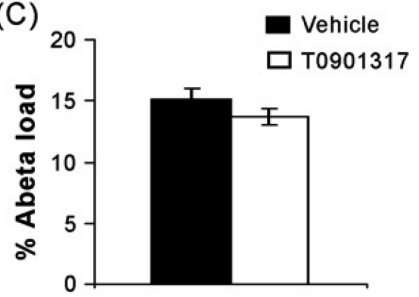

Cortex

Fig. 5. Amyloid plaque deposition in the cortex and different regions of the hippocampus.

Slices of vehicle or T0901317 treated APPSLxPS1mut mice stained for A $\beta$ (WO-2 antibody) deposition are shown; a $250 \mu \mathrm{m}$ scale bar is indicated. Magnifications (4x) of Cornu Amonis 1 (CA1) (1 and 5), CA3 (2 and 6), Dendate Gyrus (DG) and cortex (CTX) are shown below (A). The amyloid load in the cortex $(\mathrm{C})$ and different regions of the hippocampus (B) (cornu amonis 1 CA1, CA3 and DG) of T0901317 (T09) $(n=8)$ and vehicle $(n=8)$ treated APPSLxPS1mut mice are shown as percentage of surface coverage. A trend towards reduced amyloid load was observe in the $\mathrm{CA} 3(\mathrm{t}(13)=1.50 ; \mathrm{P}=0.08)$. Values are displayed as means $+/$ - SEM. 
No detectable reduction in $A \beta$ plaque load in the hippocampus of the APPSLxPS1mut mice after T0901317 administration

The A $\beta$ plaque load in the HC of the APPSLxPS1mut mice was determined after 10 weeks of treatment with T0901317. There were no significant differences in the percentage of the area covered by $\mathrm{A} \beta$ in $\mathrm{CA} 1(\mathrm{t}(13)=0.10 ; \mathrm{P}=\mathrm{n} . \mathrm{s}$.) and $\mathrm{DG}$ $(\mathrm{t}(13)=0.89 ; \mathrm{P}=\mathrm{n} . \mathrm{s})$ between the treated and untreated group. Only in the CA3, the region with the lowest number of plaques, a trend towards a decreased $\mathrm{A} \beta$ load $(\mathrm{t}(13)=1.50 ; \mathrm{P}=0.08)$ after $\mathrm{T} 0901317$ treatment was detectable (Fig. 5A \& B). Furthermore, A $\beta$ plaque load was determined in the CTX of APPSLxPS1mut mice. No significant difference was noticed between the treated and untreated group $(\mathrm{t}(13)=1.28 ; \mathrm{P}=$ n.s.) (Fig.5C). Plaque load in $\mathrm{CB}$ was not determined since no plaques could be detected in this brain region of the APPSLxPS1mut mice. Moreover, no differences in $A \beta$ plaque load were detected in the prelimbic area (corresponding to the frontal cortex in the human situation) and the anterior cingulate gyrus, both affected in $\mathrm{AD}$ (data not shown).

\section{Discussion}

Here we show that long-term pharmacological LXR-activation by T0901317 partly restores the impaired object and spatial memory in aged APPSLxPS1mut mice with major $A \beta$ deposition in their brain. In line with previous observations, T0901317 treatment upregulated levels of cholesterol precursors, lathosterol, lanosterol and desmosterol, in all brain regions examined, indicating an enhanced cholesterol turnover in the brain [6]. Abca1, Apoe and Abcg1 may be involved in the enhanced cholesterol flux, since their expression was increased upon T0901317 treatment. In contrast with our expectations, the enhanced cholesterol turnover in brain was not accompanied by a decrease in hippocampal and cortex $\mathrm{A} \beta$ load. Our data suggest that a reduction in $A \beta$ plaque load is not required for improved memory functions in this model, but that an enhanced cholesterol turnover in the brain may be involved. Although, T0901317 treatment also upregulated levels of cholesterol precursors and the expression of LXR-target genes in brain of $\mathrm{C} 57 \mathrm{BL} / 6 \mathrm{NCrl}$ mice, it did not further improve their memory functions.

Both in vitro and in vivo studies have shown that alterations in cellular cholesterol metabolism affect the processing of APP [42, 43]. LXR activation results in depletion of cholesterol from membrane lipid rafts and consequently has been reported to decrease the production of amyloid [44]. It has been suggested that the deposition of $A \beta$ is prevented by increased interstitial concentrations of HDL-like particles by binding $A \beta$ thereby preventing its deposition and/or by directing it to 
glial cells for degradation [45]. The increased levels of cholesterol precursors, together with the upregulation of the expression of Abca1, Abcg1 and Apoe by T0901317 suggest that the levels of interstitial HDL-like particles may increase due to an enhanced secretion of cholesterol. The expression of Abca1, Abcg1 and Apoe were increased by T0901317 treatment in all brain regions with the exception of the cortex of the APPSLXPS1mut mice, where the largest number of plaques is found. This suggests that the altered processing of APP and/or the deposition of A $\beta$ interferes with the LXR-pathway in the cortex.

In agreement with previous observations, 24(S)-OHcholesterol levels remained unchanged in the brain despite the upregulated cholesterol precursor levels and unchanged cholesterol levels after T0901317 administration. Therefore, alternative cholesterol clearance or metabolizing pathways involving apoE and/or HDL-like particles may exist [17, 46, 47].

It has previously been published that short term (6 days) administration of the synthetic LXR-agonist GW3965 and T0901317 to 20 weeks-old pre-plaque Tg2576 mice improved memory performance [22, 23]. Furthermore, 4 months of GW3965 administration to 12 months old $\mathrm{Tg} 2576$ is described to reduce total plaque number with $50 \%$ [22]. LXR activation reduces the $\mathrm{A} \beta_{40}$ and $\mathrm{A} \beta_{42}$ levels both in vitro and in vivo in AD-models [20, 21, 23]. Moreover, T0901317 was reported to decrease the neuronal amyloid production independent of cholesterol efflux [21]. In contrast herewith, in our experiments, no significant differences in $A \beta$ load was detected in hippocampus or cortex of the APPSLXPS1mut mice after T0901317 administration to aged APPSLXPS1mut mice. This indicates that a reduction in A $\beta$ plaque load is not essential for improvement of memory functions. APPSLXPS1mut mice display a steep increase in $A \beta$ plaque deposition from 18 months on. LXR activation may prevent the deposition of $A \beta$, but not reduce the already deposited $A \beta$. Therefore, the possibility remains that administration of T0901317 starting at the pre-plaque stage, would have prevented A $\beta$ deposition in the APPSLxPS1mut mice.

However, a dissociation between memory performance and $A \beta$ plaque load or levels was reported by others before [48, 49]. Nicotinamide restored cognition in $3 \times \mathrm{xTg}-\mathrm{AD}$ mice in parallel with a reduction in phospho-tau, but without reducing $\mathrm{A} \beta$ levels in the brain [50]. 8-Hydroxy-Quinoline was found to improve memory within hours coinciding with a decrease in soluble and insoluble interstitial $\mathrm{A} \beta$ in brain [51]. Therefore, T0901317 administration may affect specific extracellular A $\beta$ forms, which have been suggested to affect cognition [52, 53]. Yet, since T0901317 administration does not detectably affect $A \beta$ load in the hippocampus and cortex after 10 weeks, it seems unlikely that interstitial $A \beta$ levels are affected. Unfortunately, we were unable to determine the soluble $A \beta$ levels in APPSLXPS1mut mice. However, we performed an $A \beta$ plaque quantification in 
parallel to ELISAs for SDS and formic acid soluble A $\beta 40$ and A $\beta 42$ in 10 months old APPSwe/PS1dE9 mice either on a 10 weeks T0901317 or chow fed diet [54]. No differences were detected with respect to $A \beta$ plaque deposition and SDS or formic acid soluble $A \beta 40$ or $A \beta 42$ (data not shown). Despite the fact that the immunochemical data in the APPSwe/PS1dE9 mice support our immunohistological findings in the APPSLxPS1mut mice, alterations in soluble A $\beta$ in the APPSLxPS1mut mice after T0901317 diet can not be excluded.

Our data suggest that alterations in cerebral cholesterol metabolism are involved in the beneficial effects of T0901317 on memory in the APPSLxPS1mut mice. The enhanced cholesterol flux in the brain may beneficially affect a number of processes involved in learning and memory, such as the formation of new synapses, vesicle exocytosis, generation of the non-sterol isoprenoid geranylgeraniol and neurosteroids [55-59]. However, in aged C57BL/6NCrl mice with normal memory functions, the T0901317-induced enhanced cholesterol turnover does not further improve memory functions. Since T0901317 besides LXRs, also activates PXR, involvement of the latter pathway may be involved in improved memory performance [60].

Peculiarly, T0901317 treatment caused increased mortality in the APPSLxPS1mut mice, but not in the control $\mathrm{C} 57 \mathrm{BL} / 6 \mathrm{NCrl}$ group. This has not been described previously after long term treatment of Tg2576 AD mice with GW3965 [22]. Neither did we observe increased mortality after 10 weeks of T0901317 treatment of APPSwe/PS1dE9 mice (data not shown). Although the mechanisms remain speculative, a potential cause could rely in the physiological expression pattern of the knocked-in APPSL protein under the endogenous APP promoter in this specific model resulting in central as well as peripheral expression, similar to that in familial $\mathrm{AD}$ patients. This is presently under investigation.

In conclusion, T0901317 treatment can improve memory functions even in aged $\mathrm{AD}$ mice with advanced $\mathrm{AD}$ neuropathology, independently of $\mathrm{A} \beta$ plaque load. Our data suggest that an enhanced cholesterol flux may be involved in the mechanism underlying the beneficial effects of T0901317. Cerebral cholesterol metabolism may be a suitable target in the treatment and/or retardation of neurodegenerative diseases, including those other than AD. 


\section{Acknowledgements}

We thank Dr. D. Jaarsma and Prof Dr. J. Walter for critically reading the manuscript and Silvia Friedrichs and Anja Kerksiek for their excellent technical assistance. The project was financially supported by Marie Curie Early Stage Training Fellowship: Contract number MEST-CT-2005-02058. All experimental procedures concerning the used mice were approved by the local ethical committee of the Maastricht University for animal experiments according to governmental guidelines. There are no actual conflicts of interest. 


\section{References}

1. Hirsch-Reinshagen, V., B.L. Burgess, and C.L. Wellington, Why lipids are important for Alzheimer disease? Mol Cell Biochem, 2008.

2. Marx, J., Alzheimer's disease. Bad for the heart, bad for the mind? Science, 2001. 294(5542): p. 508-9.

3. Ye, S., et al., Apolipoprotein (apo) E4 enhances amyloid beta peptide production in cultured neuronal cells: apoE structure as a potential therapeutic target. Proc Natl Acad Sci U S A, 2005. 102(51): p. 18700-5.

4. Zhang, M., et al., Astrocyte-only Npc1 reduces neuronal cholesterol and triples life span of Npc1(/-) mice. J Neurosci Res, 2008.

5. Tint, G.S., et al., Markedly increased tissue concentrations of 7-dehydrocholesterol combined with low levels of cholesterol are characteristic of the Smith-Lemli-Opitz syndrome. J Lipid Res, 1995. 36(1): p. 89-95.

6. Repa, J.J., et al., Liver X receptor activation enhances cholesterol loss from the brain, decreases neuroinflammation, and increases survival of the NPC1 mouse. J Neurosci, 2007. 27(52): p. 14470-80.

7. Antoine, J.C., et al., Pathology of roots, spinal cord and brainstem in syringomyelia-like syndrome of Tangier disease. J Neurol Sci, 1991. 106(2): p. 179-85.

8. $\quad$ Milagre, I., et al., Transcriptional regulation of the human CYP46A1 brain-specific expression by Sp transcription factors. J Neurochem, 2008. 106(2): p. 835-49.

9. Corder, E.H., et al., Gene dose of apolipoprotein E type 4 allele and the risk of Alzheimer's disease in late onset families. Science, 1993. 261(5123): p. 921-3.

10. Mahley, R.W. and S.C. Rall, Jr., Apolipoprotein E: far more than a lipid transport protein. Annu Rev Genomics Hum Genet, 2000. 1: p. 507-37.

11. Haag, M.D., et al., Statins are associated with a reduced risk of Alzheimer disease regardless of lipophilicity. The Rotterdam Study. J Neurol Neurosurg Psychiatry, 2008.

12. Wolozin, B., et al., The cellular biochemistry of cholesterol and statins: insights into the pathophysiology and therapy of Alzheimer's disease. CNS Drug Rev, 2004. 10(2): p. 127-46.

13. Dietschy, J.M. and S.D. Turley, Cholesterol metabolism in the brain. Curr Opin Lipidol, 2001. 12(2): p. 105-12.

14. Lutjohann, D., Cholesterol metabolism in the brain: importance of 24S-hydroxylation. Acta Neurol Scand Suppl, 2006. 185: p. 33-42.

15. Lund, E.G., J.M. Guileyardo, and D.W. Russell, cDNA cloning of cholesterol 24-hydroxylase, a mediator of cholesterol homeostasis in the brain. Proc Natl Acad Sci U S A, 1999. 96(13): p. 7238-43.

16. Rebeck, G.W., Cholesterol efflux as a critical component of Alzheimer's disease pathogenesis. J Mol Neurosci, 2004. 23(3): p. 219-24.

17. Shafaati, M., et al., Levels of ApoE in cerebrospinal fluid are correlated with Tau and 24SOHcholesterol in patients with cognitive disorders. Neurosci Lett, 2007. 425(2): p. 78-82.

18. Schultz, J.R., et al., Role of LXRs in control of lipogenesis. Genes Dev, 2000. 14(22): p. 2831-8.

19. Whitney, K.D., et al., Regulation of cholesterol homeostasis by the liver X receptors in the central nervous system. Mol Endocrinol, 2002. 16(6): p. 1378-85.

20. Koldamova, R.P., et al., The liver X receptor ligand T0901317 decreases amyloid beta production in vitro and in a mouse model of Alzheimer's disease. J Biol Chem, 2005. 280(6): p. 4079-88.

21. Sun, Y., et al., Expression of liver X receptor target genes decreases cellular amyloid beta peptide secretion. J Biol Chem, 2003. 278(30): p. 27688-94.

22. Jiang, Q., et al., ApoE promotes the proteolytic degradation of Abeta. Neuron, 2008. 58(5): p. 68193.

23. Riddell, D.R., et al., The LXR agonist TO901317 selectively lowers hippocampal Abeta42 and improves memory in the Tg2576 mouse model of Alzheimer's disease. Mol Cell Neurosci, 2007. 34(4): p. 621-8. 
24. Zelcer, N., et al., Attenuation of neuroinflammation and Alzheimer's disease pathology by liver $x$ receptors. Proc Natl Acad Sci U S A, 2007. 104(25): p. 10601-6.

25. Plosch, T., et al., Increased hepatobiliary and fecal cholesterol excretion upon activation of the liver X receptor is independent of ABCA1. J Biol Chem, 2002. 277(37): p. 33870-7.

26. Bell, R.D., et al., Transport pathways for clearance of human Alzheimer's amyloid beta-peptide and apolipoproteins $E$ and $J$ in the mouse central nervous system. J Cereb Blood Flow Metab, 2007. 27(5): p. 909-18.

27. Mulder, M. and D. Terwel, Possible link between lipid metabolism and cerebral amyloid angiopathy in Alzheimer's disease: A role for high-density lipoproteins? Haemostasis, 1998. 28(34): p. 174-94.

28. Kohler, C., et al., Alzheimer's disease-like neuropathology of gene-targeted APP-SLxPS1mut mice expressing the amyloid precursor protein at endogenous levels. Neurobiol Dis, 2005. 20(2): p. 528-40.

29. Duff, K., et al., Increased amyloid-beta42(43) in brains of mice expressing mutant presenilin 1. Nature, 1996. 383(6602): p. 710-3.

30. Rutten, K., et al., The selective PDE5 inhibitor, sildenafil, improves object memory in Swiss mice and increases cGMP levels in hippocampal slices. Behav Brain Res, 2005. 164(1): p. 11-6.

31. Sik, A., et al., Performance of different mouse strains in an object recognition task. Behav Brain Res, 2003. 147(1-2): p. 49-54.

32. Mulder, M., et al., Low-density lipoprotein receptor-knockout mice display impaired spatial memory associated with a decreased synaptic density in the hippocampus. Neurobiol Dis, 2004. 16(1): p. 212-9.

33. Hooijmans, C.R., et al., Amyloid beta deposition is related to decreased glucose transporter-1 levels and hippocampal atrophy in brains of aged APP/PS1 mice. Brain Res, 2007. 1181: p. 93103.

34. Irizarry, M.C., et al., Abeta deposition is associated with neuropil changes, but not with overt neuronal loss in the human amyloid precursor protein V717F (PDAPP) transgenic mouse. J Neurosci, 1997. 17(18): p. 7053-9.

35. Shukla, C. and L.R. Bridges, Regional distribution of tau, beta-amyloid and beta-amyloid precursor protein in the Alzheimer's brain: a quantitative immunolabelling study. Neuroreport, 1999. 10(18): p. 3785-9.

36. Kawarabayashi, T., et al., Age-dependent changes in brain, CSF, and plasma amyloid (beta) protein in the Tg2576 transgenic mouse model of Alzheimer's disease. J Neurosci, 2001. 21(2): p. $372-81$.

37. Jansen, P.J., et al., Dietary plant sterols accumulate in the brain. Biochim Biophys Acta, 2006.

38. Lutjohann, D., et al., Profile of cholesterol-related sterols in aged amyloid precursor protein transgenic mouse brain. J Lipid Res, 2002. 43(7): p. 1078-85.

39. Heid, C.A., et al., Real time quantitative PCR. Genome Res, 1996. 6(10): p. 986-94.

40. Dixon, W.J., Analyses of extreme values. Annals of Mathematical Statistics, 1959. 21: p. 488-506.

41. Dixon, W.J., Ratios involving extreme values. Annals of Mathematical Statistics, 1959. 22: p. 6878.

42. Shobab, L.A., G.Y. Hsiung, and H.H. Feldman, Cholesterol in Alzheimer's disease. Lancet Neurol, 2005. 4(12): p. 841-52.

43. Xiong, H., et al., Cholesterol retention in Alzheimer's brain is responsible for high beta-and gamma-secretase activities and Abeta production. Neurobiol Dis, 2008. 29(3): p. 422-37.

44. $\quad$ Eckert, G.P., et al., Regulation of central nervous system cholesterol homeostasis by the liver $X$ receptor agonist TO-901317. Neurosci Lett, 2007. 423(1): p. 47-52.

45. Wahrle, S.E., et al., Overexpression of ABCA1 reduces amyloid deposition in the PDAPP mouse model of Alzheimer disease. J Clin Invest, 2008. 118(2): p. 671-82.

46. Meaney, S., et al., Novel route for elimination of brain oxysterols across the blood-brain barrier: conversion into 7alpha-hydroxy-3-oxo-4-cholestenoic acid. J Lipid Res, 2007. 48(4): p. 944-51. 
47. Wang, N., et al., ATP-binding cassette transporters G1 and G4 mediate cholesterol and desmosterol efflux to HDL and regulate sterol accumulation in the brain. Faseb J, 2007.

48. McLean, C.A., et a1., Soluble pool of Abeta amyloid as a determinant of severity of neurodegeneration in Alzheimer's disease. Ann Neurol, 1999. 46(6): p. 860-6.

49. Snowdon, D.A., Aging and Alzheimer's disease: lessons from the Nun Study. Gerontologist, 1997. 37(2): p. 150-6.

50. Green, K.N., et al., Nicotinamide restores cognition in Alzheimer's disease transgenic mice via a mechanism involving sirtuin inhibition and selective reduction of Thr231-phosphotau. J Neurosci, 2008. 28(45): p. 11500-10.

51. Adlard, P.A., et al., Rapid restoration of cognition in Alzheimer's transgenic mice with 8-hydroxy quinoline analogs is associated with decreased interstitial Abeta. Neuron, 2008. 59(1): p. 43-55.

52. Cleary, J.P., et al., Natural oligomers of the amyloid-beta protein specifically disrupt cognitive function. Nat Neurosci, 2005. 8(1): p. 79-84.

53. Townsend, M., et al., Orally available compound prevents deficits in memory caused by the Alzheimer amyloid-beta oligomers. Ann Neurol, 2006. 60(6): p. 668-76.

54. Borchelt, D.R., et al., Accelerated amyloid deposition in the brains of transgenic mice coexpressing mutant presenilin 1 and amyloid precursor proteins. Neuron, 1997. 19(4): p. 939-45.

55. Chintagari, N.R., et al., Effect of cholesterol depletion on exocytosis of alveolar type II cells. Am J Respir Cell Mol Biol, 2006. 34(6): p. 677-87.

56. Toledo, J.D., et al., Cholesterol flux between lipid vesicles and apolipoprotein AI discs of variable size and composition. Arch Biochem Biophys, 2000. 380(1): p. 63-70.

57. Mukai, H., et al., Local neurosteroid production in the hippocampus: influence on synaptic plasticity of memory. Neuroendocrinology, 2006. 84(4): p. 255-63.

58. Kotti, T., et al., Biphasic requirement for geranylgeraniol in hippocampal long-term potentiation. Proc Natl Acad Sci U S A, 2008. 105(32): p. 11394-9.

59. Marx, C.E., et al., Proof-of-Concept Trial with the Neurosteroid Pregnenolone Targeting Cognitive and Negative Symptoms in Schizophrenia. Neuropsychopharmacology, 2009.

60. Mitro, N., et al., T0901317 is a potent PXR ligand: implications for the biology ascribed to LXR. FEBS Lett, 2007. 581(9): p. 1721-6. 



\section{Chapter IV}

\section{Irreversible accumulation of plant sterols in the brain}




\section{Chapter IV}

\section{Irreversible Accumulation of Plant Sterols in the Brain}

Tim Vanmierlo ${ }^{\mathrm{a}, \mathrm{b}, \mathrm{g}}$, Oliver Weingärtner ${ }^{\mathrm{c}}$, Susanne van der Pol $^{\mathrm{d}}$, Constanze Husche ${ }^{\mathrm{a}}$, Irfan Tamboli ${ }^{\mathrm{e}}$, Anja Kerksiek ${ }^{\mathrm{a}}$, Silvia Friedrichs ${ }^{\mathrm{a}}$, Eric Sijbrands ${ }^{\mathrm{f}}$, Harry Steinbusch $^{\mathrm{b}, \mathrm{g}}$, Ulrich Laufs ${ }^{\mathrm{c}}$, Michael Böhm ${ }^{\mathrm{c}}$, Elga de Vries ${ }^{\mathrm{d}}$, Jochen Walter ${ }^{\mathrm{e}, \mathrm{g}}$, Monique Mulder ${ }^{\mathrm{f}}$, Dieter Lütjohann ${ }^{\mathrm{a}, \mathrm{g}}$

a Institute of Clinical Chemistry and Pharmacology, University of Bonn, Bonn, Germany; b Department of Neuroscience, Maastricht University, Maastricht, The Netherlands; c Klinik für Innere Medizin III, Kardiologie, Angiologie und Internistische Intensivmedizin, Universitätsklinikum des Saarlandes, Homburg/Saar, Germany; d Department of Molecular Cell Biology and Immunology, VU University Medical Center, Amsterdam, The Netherlands; e Department of Neurology, University of Bonn, Bonn, Germany; f Department of Internal Medicine, Division of Pharmacology, Vascular and Metabolic Diseases, Erasmus Medical Center, Rotterdam, The Netherlands; ${ }^{\mathrm{g}}$ EURON (European Graduate School for Neuroscience)

Submitted 


\section{Abstract}

Plant sterols such as sitosterol and campesterol are frequently administered as functional food additives in the prevention of atherosclerosis, although hard endpoint studies are lacking. Recently, it became clear that plant sterols can accumulate in murine brains. We questioned if the accumulation would be reversible and if plant sterol accumulation can modulate cholesterol related pathology in Alzheimer's disease (AD).

After being fed a plant sterol ester enriched diet for a period of 6 weeks, C57BL/6NCrl mice displayed significantly increased serum, liver and brain plant sterol concentrations. Brain plant sterol levels did however not decrease after subsequently feeding the mice a plant stanol ester enriched diet for a period of 6 months that almost completely depletes them from plant sterol uptake. In contrast, serum and liver plant sterol concentrations were significantly reduced. Thus plant sterol accumulation in the brain is irreversible. Campesterol accumulated both in vitro and in vivo more efficiently across the blood-brain barrier than sitosterol when compared to the serum plant sterol concentrations. Plant sterols were found to be incorporated within the detergent resistant membranes. In relation to $\mathrm{AD}$, in vitro, only sitosterol reduced the secretion of the toxic amyloid-beta fragment, by reducing the maturation of the amyloid precursor protein overexpressing Neuro2A cells.

In conclusion, accumulation of plant sterols in the brains of C57BL/6NCrl mice is irreversible with a higher flux for campesterol than for sitosterol. Plant sterols accumulate within the lipid rafts of brain cell membranes, but only sitosterol reduces amyloid precursor protein processing.

\section{Introduction}

Plant sterols are in contrast to cholesterol solely derivable from the diet. They differ structurally from cholesterol only by an additional methyl or ethyl group at C24 and/or a double bond at $\mathrm{C} 22(\Delta 22)$. The most prevalent plant sterols are campesterol (methyl C24), sitosterol (ethyl C24), brassicasterol (methyl C24, $\Delta 22$ ), and stigmasterol (ethyl C24, $\Delta 22)[1,2]$. A high plant sterol intake $(2-2.5 \mathrm{~g} /$ day) leads to reduced serum and low density lipoprotein (LDL) cholesterol levels $(\sim 12 \%)[3,4]$. Therefore, plant sterols are frequently applied as functional food additives in the prevention of atherosclerosis and cardiovascular diseases (CVD) without prescription [5]. Administration of plant sterols results besides reduced serum and LDL cholesterol concentrations, in an elevation of serum plant sterol 
concentrations [6]. Since hard end-point studies are lacking [7], as well as insights in the underlying molecular mechanisms [8] and adverse events upon plant sterol administration in animal studies are increasingly being reported [9], the administration of plant sterols is a current topic of debate [10].

In contrast to the peripheral tissues, all cholesterol within the central nervous system (CNS) is synthesized in situ, since circulating cholesterol is assumed to be prevented from entering the brain by the presence of the blood-brain barrier (BBB) $[11,12]$. Recently, we reported that plant sterols, in contrast with cholesterol, can accumulate in the brain of ATP binding cassette G5 (ABC)G5-deficient mice, a model for phytosterolemia (Jansen et al. 2006). ABCG5 and ABCG8 act as functional heterodimer transporters at the apical membranes of enterocytes and hepatocytes where they resecrete plant sterols into the intestinal lumen and bile, respectively [13]. However, some plant sterols still end up in the serum and small amounts reach the brain. Accumulating evidence suggests a key role for a disturbed brain cholesterol homeostasis in the development and proceeding of Alzheimer's disease (AD) [14-16]. A reduction in total membrane cholesterol concentrations and more in particular in lipid rafts has been shown to reduce the production of amyloid- $\beta(\mathrm{A} \beta)$ by disrupting the splicing of its larger amyloid precursor protein (APP) by the $\gamma$-secretase complex $[17,18]$. Mild cholesterol depletion consequently impairs APP trafficking to the membrane, making it less prone for cleavage within the lipid rafts or detergent resistant membranes (DRMs) [17]. Moreover, processing of APP into A $\beta$ is requiring cholesterol rich DRM microdomains [19]. Plant sterols are known to be organized in lipid rafts within plant cell membranes [20-22]. Therefore, we first assessed if the plant sterols that accumulated in the mouse brain are being resecreted from the brain after feeding a plant stanol enriched / plant sterol depleted diet. In addition, we hypothesized that plant sterols can modulate AD pathology by interfering with the membrane cholesterol metabolism in an in vitro setup.

\section{Materials and Methods}

Animals

All experiments were approved by the local ethical committee for animal experiments of the Universitätsklinikum des Saarlandes according to the institutional and governmental guidelines. C57BL/6NCrl male mice were purchased from Charles River Laboratories GmbH (Sulzfeld, Germany). At 12 weeks (time 
point 1 ), mice ( $\mathrm{n}=29)$ were randomly divided into two groups. One group was fed a $2 \%$ plant sterol ester (PSE) enriched diet (60\% sitosterol, 30\% campesterol, and $10 \%$ stigmasterol) $(\mathrm{n}=14)$ whereas the other group was fed a control diet $(0.015 \%$ plant sterol: $65 \%$ sitosterol, $30 \%$ campesterol, and $5 \%$ stigmasterol) $(\mathrm{n}=15)$ (figure 1). After 6 weeks of diet, 6 animals per group were sacrificed (time point 2). Subsequently, the remaining animals in the group previously fed a plant sterol diet $(\mathrm{n}=8)$ were now fed a $1 \%$ plant stanols enriched diet (85\% sitostanol, $15 \%$ campestanol). The diet of the remaining animals in the control group ( $\mathrm{n}=9)$ was prolonged for 6 more months (containing $0.0015 \%$ plant stanols: $55 \%$ sitostanol and $45 \%$ campestanol) (time point 3). All animals were sacrificed at the end of the experiment with an overdose of ketamine $(1 \mathrm{~g} / \mathrm{kg} /$ body weight $)$ and Xylazine (100 $\mathrm{mg} / \mathrm{kg} /$ body weight). Liver, serum and brain hemispheres were snap frozen in liquid nitrogen and stored at $-80^{\circ} \mathrm{C}$ awaiting further analysis. Food sterol concentrations were verified with gas chromatograph/mass spectrometry (GC/MS).

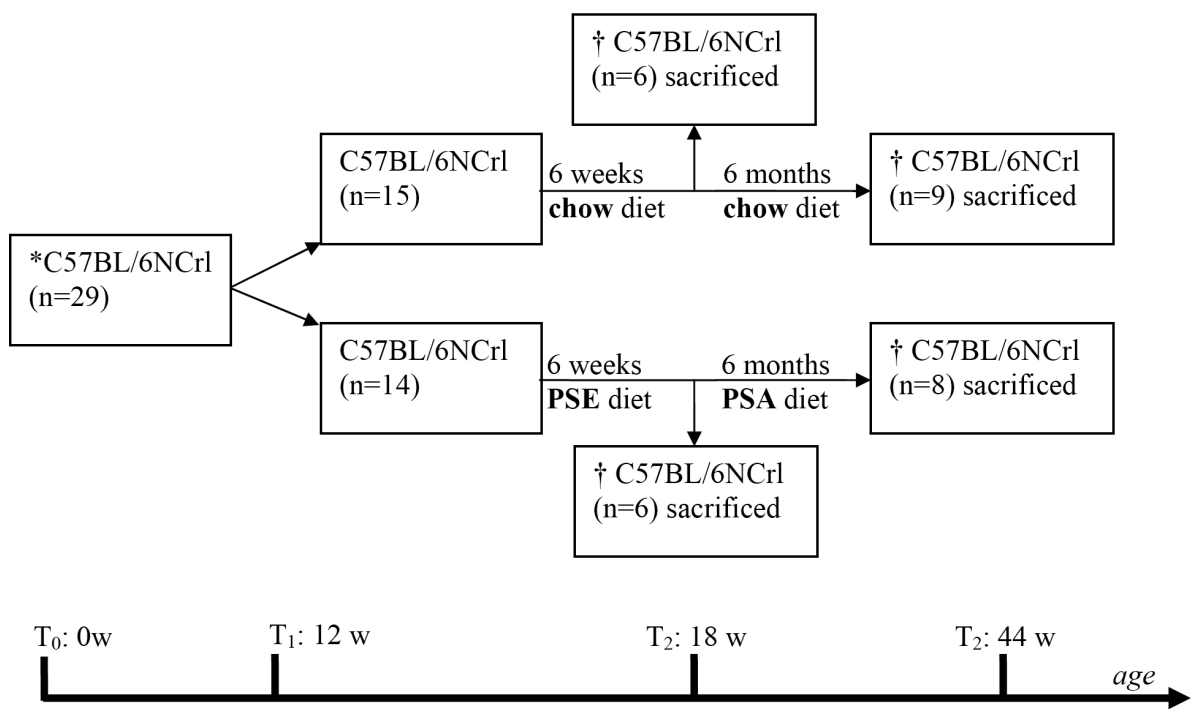

Fig.1. Experimental scheme of $\mathrm{C} 57 \mathrm{BL} / 6 \mathrm{NCrl}$ feeding experiment.

At 12 weeks $\mathrm{C} 57 \mathrm{BL} / 6 \mathrm{NCrl}(\mathrm{n}=29)$ were randomly subdivided in two groups respectively fed a regular chow diet $(n=15)$ or a plant sterol ester enriched diet (PSE) $(n=15)$. After 6 weeks, 6 animals per group were sacrificed. The remaining animals on the regular chow diet $(n=9)$ were further fed the normal chow diet. The remaining mice from the PSE group $(n=8)$ were now fed a plant stanol ester (PSA) enriched diet. After 6 months of the second feeding term, all animals were sacrificed. 


\section{Chemical reagents}

d6-Sitosterol/d6-campesterol (55\%-45\%) (Sugaris GmbH, Münster, Germany), d6cholesterol (99.0\%) (CDN isotopes, Pointe-Claire, Quebec, Canada), epicoprostanol (99.9\%) Sigma, St. Louis, MO, USA), sitosterol (98\%) (Alltech Associates inc., Deerfield, IL, USA), campesterol (81\% campesterol, 11\% sitosterol, 8\% stigmasterol), stigmasterol (99.7\%), cholesterol (99\%) (SigmaAldrich Chemie, Steinheim, Germany) were quantified and tested on purity against $5 \alpha$-cholestan $(\mu \mathrm{g} / \mu \mathrm{l})$ (Serva Electrophoresis $\mathrm{GmbH}$, Heidelberg, Germany) using gas chromatography flame ionization detection (GC/FID) and GC/MS scan method. Sterols were dissolved in $100 \%$ ethanol to obtain a $10 \mathrm{mM}$ stock solution.

Analyses

\section{Cell culture experiments}

The human astrocytoma CCF-STTG1 and neuroblastoma SHSY5Y cell lines, purchased from European Collection of cell Cultures (CACC, Salisbury, UK) were cultured in 10\% FCS (EU approved origin, Gibco, Invitrogen $\mathrm{GmbH}$, Karlsruhe Germany) containing 1:1 Dulbecco's Modified Eagle's Medium and F-12 Nutrient mixture (ham) (DMEM/F-12) (Gibco, Invitrogen GmbH, Karlsruhe Germany) including 100 units penicillin and $100 \mu \mathrm{g}$ streptomycin per $\mathrm{ml}$ (pen/strep from Gibco, Invitrogen GmbH, Karlsruhe Germany). The hCMEC/D3 cell line is a human adult brain endothelial cell line, described to be an excellent cell line for in vitro studies of the BBB [23]. OLN-93 cells (oligodendroglioma derived from primary rat brain glial cultures) were kindly provided by Dr Richter-Landsberg, Oldenburg, Germany [24]. The mouse neuroblastoma (N2A) cells were cultured in 10\% FCS DMEM with 100u pen/ 100 $\mathrm{g}$ strep/ml. N2A cells were transfected with pcDNA3.1 vector (Invitrogen $\mathrm{GmbH}$, Karlsruhe Germany) containing the APP gene over $6 \mathrm{~h}$ using OPTI-MEM ${ }^{\circledR}$ reduced serum medium (Gibco, Invitrogen $\mathrm{GmbH}$, Karlsruhe Germany) and Lipofectamine 2000 (Invitrogen GmbH, Karlsruhe Germany) according to the manufacturers prescriptions. Stable transfectants were selected in 10\% FCS DMEM supplemented with $0.5 \mathrm{mg} / \mathrm{ml}$ G418 (Gibco, Invitrogen $\mathrm{GmbH}$, Karlsruhe Germany). The pcDNA3.1 vector was encoding the gene resistance to the neomycin analog, G418.

\section{Western Blot Analysis and antibodies}

Cell lysates $(25 \mu \mathrm{g} /$ lane) were dissolved in RIPA buffer (10mM Tris, $150 \mathrm{mM} \mathrm{NaCl}$, $1 \mathrm{mM}$ EDTA, 1\% Triton-X-100, 0,1\% deoxycholic acid) and subsequently mixed 1:1 with laemmli sample buffer (2x) (Biorad laboratories, Hercules, CA, USA). $15 \mu 1$ Supernatant aliquots were 1:1 mixed with laemmli sample buffer. Samples 
were subjected to dodecyl sulphate- 7.5 or $10 \%$ polyacrylamide gel electrophoresis 7.5 or $10 \%$ SDS-PAGE and then transferred to Trans-Blot ${ }^{\circledR}$ transfer pure nitrocellulose medium (Biorad laboratories, Hercules, CA, USA). After 1h blocking at room temperature in blotting $5 \%$ grade blocker non-fat dry milk (Biorad laboratories, Hercules, CA, USA) in Tris buffered saline (Biorad laboratories, Hercules, CA, USA) - Triton $0.1 \%$ (Biorad laboratories, Hercules, CA, USA), the membranes were incubated for $1 \mathrm{~h} 15$ min with polyclonal rabbit anti-APP Nterminal antibody $(1: 1000,5313$ described before $[25,26])$ at room temperature. The membranes were then incubated with a peroxidase-conjugated donkey antirabbit (1:20000, Santa Cruz biotechnologies, Santa Cruz, CA, USA) secondary antibodiy after which the results were visualized using AceGlow ${ }^{\mathrm{TM}}$ chemiluminicense (Peqlab biotechnology $\mathrm{GmbH}$, Erlangen, Germany). Luminicent signal was detected by Chemicapt 5000 detector and software (Peqlab biotechnology GmbH, Erlangen, Germany).

\section{AB ELISA}

$A \beta 40$ and $A \beta 42$ were detected immunochemically using ELISA kits against human $\mathrm{A} \beta 40$ and AB42 (Invitrogen, \#KHB3482 and \#KHB3544, Camarillo, CA, USA) in N2A and SHSY5Y cell lysates and supernatant according to the manufacturers instructions.

\section{In vitro $B B B$ model}

hCMEC/D3 were seeded at confluency onto collagen-coated Costar Transwell filter setup (pore-size $0.4 \mu \mathrm{m}$; Corning Incorporated) in growth medium containing $2.5 \%$ FCS and grown for 4 days $(200 \mu 1$ upper compartment, $800 \mu 1$ medium in the lower compartment). Paracellular permeability of human brain endothelial cell monolayers was analyzed as described previously [27]. In addition, one hour before harvesting the cells in the presence of $15 \mu \mathrm{M}$ d6-sitosterol/d6-campesterol or d6cholesterol, paracellular permeability was validated by adding fluorescein isothiocyanate coupled dextran (FITC-dextran: $150 \mathrm{kDa}, \mu \mathrm{g} / \mu \mathrm{l}$ in culture medium; Sigma-Aldrich) in the upper compartment. After $24 \mathrm{~h}$ of co-culture, $10 \mu 1$ per sample was collected from the upper and lower chambers for measurement of fluorescence intensity using a FLUOstar Galaxy microplate reader (BMG Labtechnologies), excitation $485 \mathrm{~nm}$ and emission $520 \mathrm{~nm}$, at gain 64 . The average $1 \mathrm{~h}$ flux of FITC-dextran through the endothelial monolayer was calculated and extremes were excluded from further analyses based on Dixon's principles of exclusion for extreme values [28, 29]. Viability of the hMEC/D3 cells was determined by Water-soluble tetrazolium (WST) assay to be unaltered after incubation of different concentrations of d6-sitosterol/d6-campesterol or d6- 
cholesterol $(0,5,10,15,20,25$ and $30 \mu \mathrm{M})$ for different incubation times $(6 \mathrm{~h}, 24 \mathrm{~h}$ and 48h) (data not shown). Subsequently, the trans-endothelial flux experiment was repeated with confluent primary astrocytes at the bottom of the lower compartment in the endothelial-Transwell ${ }^{\circledR}$ setup to further strengthen the endothelial tight junctions, restricting the permeability of the BBB and improving the blood-brain barrier model [30]. Plant sterol and cholesterol flux was measured $24 \mathrm{~h}$ after incubation by sterol extraction and quantification in supernatant, endothelial cell layer, astrocytes cell layer, and transfluent of 4 pooled chambers by GC/MS analyses.

\section{Efflux studies}

CCF-STTG1 (astrocytes), SHSY5Y (neurons) and OLN-93 (oligodendrocytes) cells were cultured in culture medium supplemented with $10 \%$ fetal calf serum until $90 \%$ confluency. After washing cells with $1 \mathrm{x}$ PBS, cells were incubated with $0.5 \%$ FCS medium containing either $15 \mu \mathrm{M}$ d6-cholesterol or d6-sitosterol/d6-campesterol. After $24 \mathrm{~h}$ the medium was discarded. Cells were washed three times with PBS and serum free medium containing either $25 \mu \mathrm{g} / \mathrm{ml} \mathrm{HDL}$ (isolated as described previously [31] from a freshly constituted serum pool of 4 individuals) or vehicle control $(0.2 \%$ BSA in sterile PBS) were added for $6 \mathrm{~h}$. Medium and cells were collected separately and analysed by GC/MS for d6-cholesterol and respectively d6sitosterol/d6-campesterol content.

\section{Detergent Resistant Membrane isolation from confluent cell lines}

SHSY5Y cells were cultured till confluency in 10\% FCS containing medium in T75 flasks. Cells were then 3:2000 stimulated using the $10 \mathrm{mM}$ sterol stock concentrations (sitosterol, campesterol, stigmasterol, mixture, cholesterol or vehicle (ethanol)) for $24 \mathrm{~h}$ in $0.5 \%$ FCS containing medium to obtain a $15 \mu \mathrm{M}$ end concentration. Lipid rafts or detergent resistant membranes (DRMs) were isolated as described before [32]. In brief, cells were washed with ice cold phosphatebuffered saline (0.15 M NaCl, $20 \mathrm{mM} \mathrm{NaH2PO4,} \mathrm{and} 2 \mathrm{mM} \mathrm{Na2HPO4,pH} \mathrm{7.4)}$ twice. Cells were subsequently scraped in $1.5 \mathrm{ml}$ ice cold TNE buffer ( $25 \mathrm{mM}$ Tris, pH 7.4, $150 \mathrm{mM} \mathrm{NaCl}$, and $2 \mathrm{mM}$ EDTA) containing "complete" protease inhibitor mix (Roche Diagnostics GmbH, Mannheim, Germany), and disrupted by mechanical stress: 15 strokes through a $25-\mathrm{G}$ needle. Nuclei were pelleted by low speed centrifugation (20 $\mathrm{min}, 1,000 \mathrm{~g}, 4^{\circ} \mathrm{C}$ ) and discarded. Membranes were pelleted from the supernatant at high speed centrifugation $\left(90 \mathrm{~min}, 17,000 \mathrm{~g}, 4^{\circ} \mathrm{C}\right)$. The membrane pellet was then dissolved in $1.5 \mathrm{ml} 1 \%$ CHAPSO (Roche Diagnostics GmbH, Mannheim, Germany) containing "complete" protease inhibitor mix (Roche Diagnostics GmbH, Mannheim, Germany) and incubated on ice for 
$30^{\prime}$. The CHAPSO cell extracts were mixed with $2.5 \mathrm{ml}$ TNE buffer containing $72 \%$ sucrose to yield a final concentration of $45 \%(\mathrm{w} / \mathrm{v})$ sucrose and laid at the bottom of $12 \mathrm{ml} \mathrm{Beckman}$ ultracentrifuge tube. $4 \mathrm{ml}$ of TNE buffer containing $35 \%$ and $5 \%$ sucrose were successively and carefully laid over the CHAPSO cell extracts. The samples were spun at $4^{\circ} \mathrm{C}$ for $19 \mathrm{~h}$ at $197,000 \mathrm{~g}$ in the SW41 TI rotor (Beckman Coulter GmbH, Krefeld, Germany). Fractions were carefully collected in $1 \mathrm{ml}$ volumes from top to bottom to yield a total of 12 fractions. The low buoyant DRM fraction was settled at the interface between the 35 and $5 \%$ sucrose as amorphous white material visible to the naked eye that were collected in fractions 4 and 5 . DRMs (density: $1.09-1.13 \mathrm{~g} / \mathrm{cm}^{3}$ [33]) were confirmed by densitometry using a DMA48 density meter (Anton Paar GmbH, Graz, Austria), on flotellin-1 expression by western blot, and cholesterol content by GC/MS (data not shown). The nonDRM fractions were confirmed to be in fraction 8-10. All individual fractions were subjected to sterol extraction preceding gas chromatography - mass spectrometry (GC/MS).

\section{Preparation of total membrane fraction of mouse brain hemispheres}

Snap frozen wet mouse brain hemispheres were weighted (mg) preceding the homogenization as described in Parkin et al. $1.5 \mathrm{ml}$ Homogenization buffer (150 $\mathrm{mM} \mathrm{NaCl}, 20 \mathrm{mM} \mathrm{Na} 2 \mathrm{HPO}_{4}, 2 \mathrm{mM} \mathrm{NaH} \mathrm{PO}_{4}, 1 \mathrm{mM}$ EDTA, and 20\% (vol/vol) glycerol, $\mathrm{pH}$ 7.4) was added to each hemisphere. 2 Cycles of $30 \mathrm{~s}$ at $6,500 \mathrm{rpm}$ in the Precellys24 homogenizer (Bertin technologies, Montigny-le-Bretonneux, France), were separated by 5 min cooling on ice. After homogenization, the samples were diluted 1:4 in homogenization buffer and centrifuged at 5,000 $\mathrm{g}$ for $20 \mathrm{~min}$. The pellet was discarded and the supernatant was centrifuged for $90 \mathrm{~min}$ at 17,000 $g$. The resultant membrane pellet was resuspended in $1.5 \mathrm{~mL}$ TNE buffer containing $1 \%$ CHAPSO (Roche Diagnostics GmbH, Mannheim, Germany) and incubated on ice for $30 \mathrm{~min} .12 \times 1 \mathrm{ml}$ fractions were carefully isolated top till bottom and DRMs were confirmed to be in fraction 4 and 5 by densitometry, cholesterol content and Flotillin-1 protein expression as depicted in the DRM isolation from confluent cell lines section of the materials and methods. Fractions were further subjected to GC/MS analyses for detection of plant sterol concentrations.

\section{Sterol Profile Determination}

Wet weights of hemispheres and protein concentrations of brain homogenates were determined in order relate sterol content to wet weight or protein concentration. Sterol contents of fractions generated from brain homogenates, total brain homogenates, cell lysates, fractions generated by sucrose gradient ultracentrifugation, total medium and cell lysates from the efflux experiment were 
determined by gas chromatography/mass spectrometry as extensively described previously $[34,35]$.

\section{Statistical Analysis}

All statistical analyses were performed using GraphPad Prism 4 ${ }^{\mathrm{TM}}$. The sterol data in serum, liver and brain were analyzed using the 1-way ANOVA with a post hoc Bonferroni's Multiple Comparison Test. Extreme values were excluded by means of Dixon's principles of exclusion of extreme values [28, 29]. Significance levels were determined on \#, §, *: $\mathrm{P}<0.05, \# \#, \S \S, * *: \mathrm{P}<0.01$ or \#\#\#, $\S \S, * * *: \mathrm{P}<0.001$.

\section{Results}

Dietary administration of plant sterols increased brain plant sterol levels irreversibly

C57BL/6NCrl mice were either fed a normal chow diet $(\mathrm{NC})(\mathrm{n}=15)$ or were challenged with a $2 \%$ plant sterol esters enriched diet (PSE) $(n=14)$ for a period of 6 weeks (Fig1). In line with several previous reports [36, 37], the PSE fed group displayed significantly increased sitosterol and campesterol concentrations in serum, liver and brain in comparison to the NC group (Fig2.A-C). Campesterol accumulated 1.2 fold more efficient in brain in comparison with sitosterol in relation to serum concentrations.

Brain plant sterol levels remained unchanged even after feeding the mice a $1.5 \%$ plant stanol ester enriched diet (PSA) for 6 months following the 6 weeks PSE diet (Fig.2C), while sitosterol and campesterol concentrations were significantly reduced in comparison to both 6 weeks $\mathrm{NC}$ and to the additional 6 months $\mathrm{NC}$ in serum (sitosterol: $\mathrm{P}<0.001$ and campesterol: $\mathrm{P}<0.001$; Fig. $2 \mathrm{~A}$ ) and liver (sitosterol: $\mathrm{P}<0.001$ and campesterol: $\mathrm{P}<0.001$; Fig.2B). Brain cholesterol and sitostanol were comparable in all groups, while brain campestanol increased two-fold upon PSA diet, still being 300-fold lower in concentration compared to campesterol (data not shown). Together, these results strongly indicate that plant sterols entering the brain in a dose dependent way accumulate almost irreversibly, even though plant sterol concentrations in serum were strongly reduced over the 6 months PSA diet. 


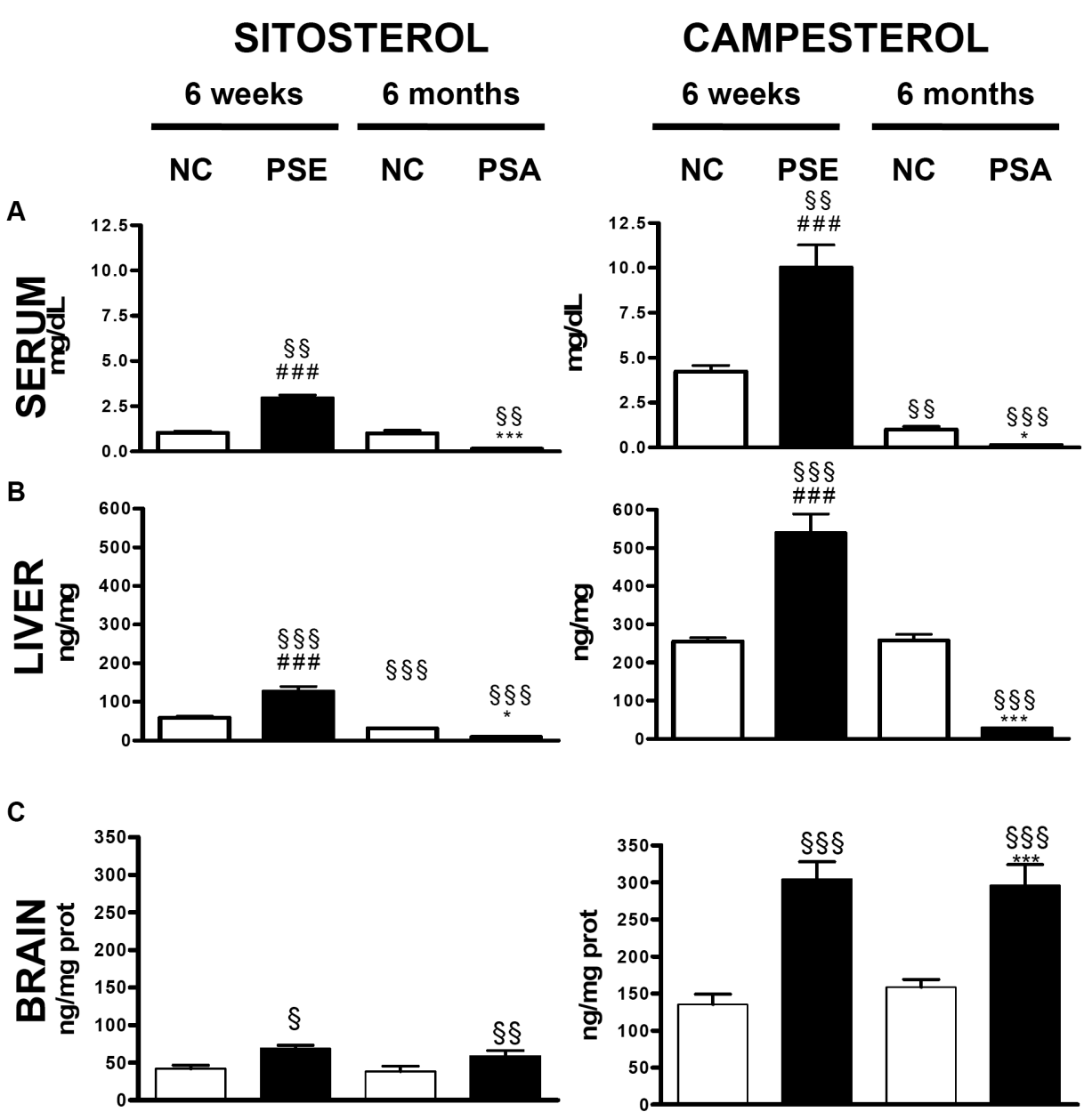

Fig.2. Plant sterol concentrations in serum, liver and brain with or without plant sterol or plant stanol administration.

12 Weeks old C57BL/6NCrl mice were fed either a normal chow (NC) $(\mathrm{n}=15)$ or a plant sterol enriched chow (PSE) $(n=14)$. After 6 weeks, 6 animals per group were sacrificed. The remaining animals on $\mathrm{NC}(\mathrm{n}=9)$ were further fed $\mathrm{NC}$ and the animals previously fed PSE were now fed a plant stanol enriched chow (PSA) for 6 more months. Concentrations of sitosterol and campesterol in serum (mg/dl) (A), liver (ng/mg dry weight) (B), and brain (ng/mg protein) (C) are displayed as mean +/- SEM. PSE leads to elevated plant sterols in the serum (A), liver (B) and brain (C). PSA reduces serum and liver plant sterol concentrations, but not brain plant sterol concentrations. ANOVA, Bonferroni Post Hoc: "§": compared to 6weeks NC fed C57BL/6/6NCrl mice and Student t-tests: “\#”: PSE versus PSA, “*”: 6 months PSA versus 6 months NC. §,*: $\mathrm{P}<0.05, \S \S: \mathrm{P}<0.01, \S \S \S$, \#\#\#,***: $\mathrm{P}<0.001$ 
Dietary administration of plant sterols increased brain plant sterol concentrations predominantly in detergent resistant membranes (DRM)

Considering the functional importance of cholesterol in neuronal membranes, the distribution of plant sterols within the membranes was determined. Membranes were isolated from brain homogenates of mice either fed a PSE enriched or a NC diet for 6 weeks. Both NC and PSE diet displayed higher levels of plant sterols in DRM than in the non-DRM fractions (sitosterol NC: DRM vs non-DRM: $\mathrm{P}<0.001$, sitosterol PSE: DRM vs non-DRM P $<0.001$, campesterol NC: DRM vs non-DRM: $\mathrm{P}<0.001$ and campesterol PSE: DRM vs non-DRM $\mathrm{P}<0.001$; Fig.3A-D). Moreover, after PSE diet, plant sterols accumulated in significantly higher amounts in the DRM fractions, but not in the non-DRM fractions in comparison to NC (sitosterol DRM: NC vs PSE: $\mathrm{P}<0.05$ and campesterol DRM: NC vs PSE: $\mathrm{P}<0.001$; Fig.3AD). Therefore, these data show that plant sterols accumulate in a dose dependent manner within the DRM fractions of the brain cell membranes.

A

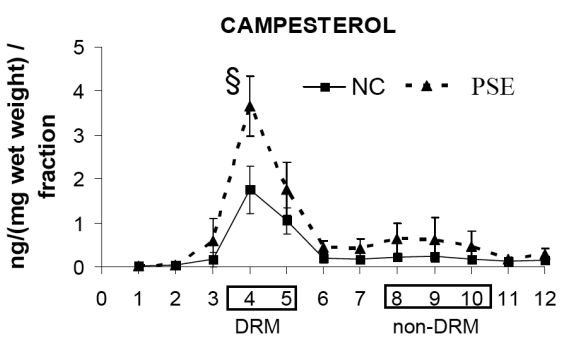

C

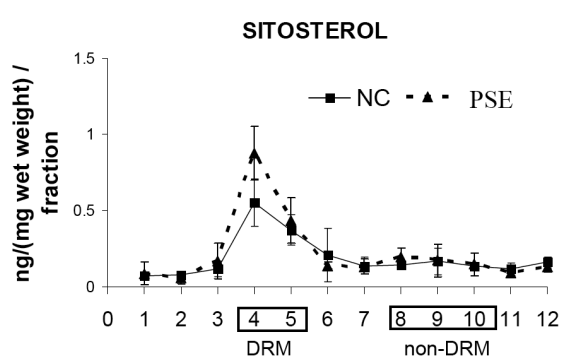

E

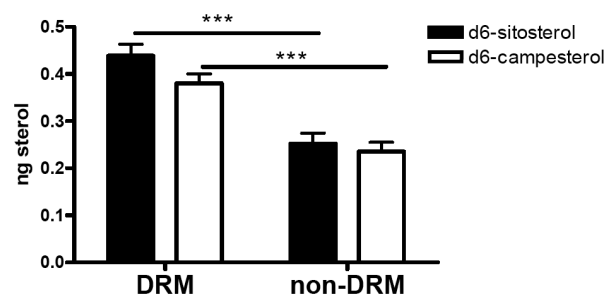

B

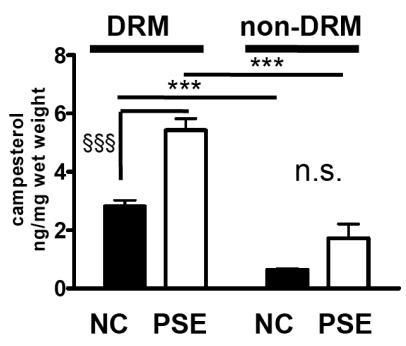

D

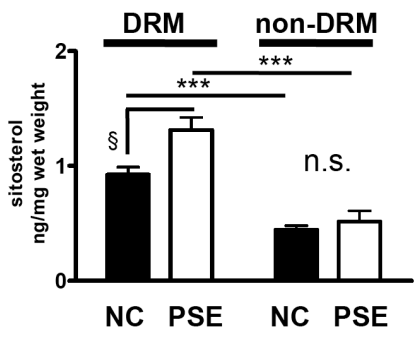


Figure 3. Plant sterol concentrations in homogenates and DRM fractions of mice brains, with or without plant sterol or plant stanol administration.

12 Weeks old C57BL/6NCrl mice were fed either a normal chow (NC) $(\mathrm{n}=15)$ or a plant sterol enriched chow (PSE) $(n=14)$. After 6 weeks, 6 Animals per group were sacrificed and sitosterol (A) and campesterol (B) concentrations were determined in $12 \times 1 \mathrm{ml}$ (top-bottom) density fractions of the membranes ( $\mathrm{ng} /$ fraction/mg wet weight). As depicted in the materials and methods section, membrane fractions were subdivided in detergent resistant membrane fractions (DRMs) and non-DRMs were validated by densitometry, cholesterol analysis and western blot for Flotillin-1, corresponding respectively to fraction 4 and 5 (DRM) and 8, 9 and 10 (non-DRM). Sitosterol (B) and Campesterol (D) DRM and non-DRM fraction masses were added up. PSE administration leads to significantly elevated plant sterols concentrations in DRMs, but not in non-DRM regions of the brain membranes. The increased accumulation of campesterol and sitosterol in DRM compared to non-DRM was subsequently validated by $24 \mathrm{~h}$ administration $(n=5)$ of $10 \mu \mathrm{M}$ d6-sitosterol/d6-campesterol $(55 \%-45 \%)$ mixture to a neuroblastoma cell line (SHSY5Y) (E). *: DRM compared to non-DRM, §: PSE compared to NC fed C57BL/6/6NCrl mice; $\S: \mathrm{P}<0.05, \S \S \S, * * *: \mathrm{P}<0.001$; n.s.: not significant.

Campesterol is more efficient than sitosterol in traversing an endothelial monolayer In order to examine if sitosterol and campesterol traverse the endothelial cell layer of the BBB or accumulate within these cells d6-sitosterol/d6-campesterol and respectively d6-cholesterol were added to the apical site of a polarized confluent human brain endothelial hMEC/D3 monolayer in an in vitro Transwell@ setup. After 24h, the internalization of all sterols was comparable (Fig.4A). However, the subsequent d6-cholesterol flux through the endothelial monolayer was \pm 5 fold more efficient than for d6-sitosterol and d6-campesterol $(\mathrm{P}<0.0001)(0.97 \% \pm 0.10$; Fig.4B). Despite a comparable apical uptake of d6-sitosterol and d6-campesterol, d6-campesterol was 1.4 fold more efficiently resecreted at the basolateral side of the endothelial cells than $\mathrm{d} 6$-sitosterol $(0.22 \% \pm 0.04$ vs $0.16 \% \pm 0.03$ : $\mathrm{P}=0.016$; Fig. $2 \mathrm{~B})$. In the presence of the primary astrocytes, we found a two-fold higher sterol flux through the endothelial monolayer, displaying similar sterol ratios (Fig.4C) One third of the sterols in the lower chamber were incorporated by the astrocytes (d6cholesterol: $40 \pm 7 \%$, d6-campesterol: $34 \pm 6 \%$ and d6-sitosterol: $29 \pm 6 \%$ ). These data show an endothelial selective BBB crossing, depending on the molecular complexity of the sterol side chain.

Plant sterols accumulate in detergent resistant membranes in cultured brain cells Plant sterol and cholesterol uptake and efflux to HDL were determined using different cerebral cell derived cell lines. Neuronal cells incorporated significantly less plant sterols and also cholesterol than glial cells (olgidendrocytes and astrocytes) both after $6 \mathrm{~h}$ and $24 \mathrm{~h}$ incubation (Table I). Slightly, but not significantly more cholesterol than plant sterols was taken up by all cell lines. Sterol efflux in the neuronal cell line could not be enhanced by external acceptor HDL. The percentage 
of secreted plant sterols was slightly higher than for cholesterol. However, when the percentages were corrected for absolute uptake values, absolute amounts in $\mu \mathrm{g}$ secreted did not differ between cholesterol and plant sterols.

After administration of $10 \mu \mathrm{M}$ d6-sitosterol/d6-campesterol (55\%-45\%) mixture, both d6-sitosterol $(\mathrm{P}<0.001)$ and $\mathrm{d}$-campesterol $(\mathrm{P}<0.001)$ were found to accumulate preferentially in the cholesterol rich DRMs rather than in the phospholipid enriched non-DRM fractions (Fig.3E). The ratio of both plant sterols of the administered mixture was maintained within each fraction measured and the accumulation paralleled the accumulation administered d6-cholesterol (data not shown).

Together these data show that plant sterols are handled likewise within the individual cerebral cell lines and subsequently accumulate within the DRMs.

A

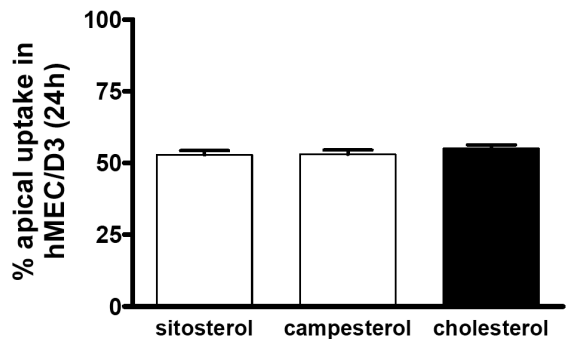

C

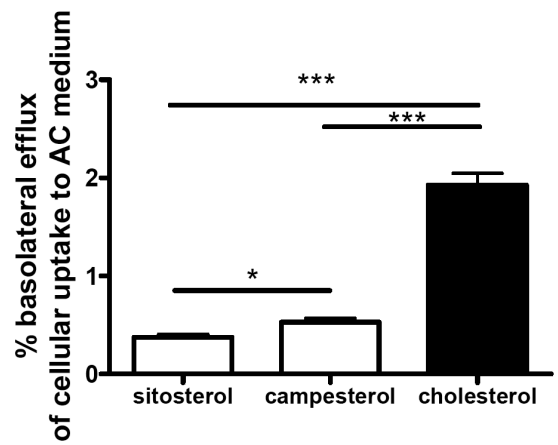

B

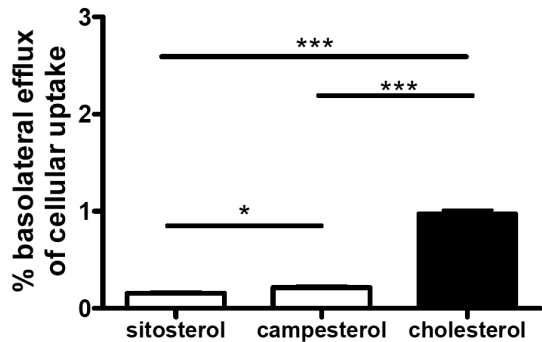

Figure 4. Apical uptake and subsequent basolateral efflux of plant sterols and cholesterol in a human endothelial cell line in a Transwell ${ }^{\circledR}$ setup.

hMEC/D3 endothelial were grown till confluency on a Transwell ${ }^{\circledR}$ permeable support $0.4 \mu \mathrm{m}$ polycarbonate membrane $6.5 \mathrm{~mm}$ insert in the absence $(\mathrm{B})$ or presence $(\mathrm{C})$ of confluently grown primary astrocytes $(\mathrm{AC})$ at the bottom of the lower chamber. $15 \mu \mathrm{M} \mathrm{d} 6$-sitosterol/d6-campesterol $(n=6)$ and respectively $15 \mu \mathrm{M}$ d6-cholesterol $(n=6)$ were added for $24 \mathrm{~h}$ to the upper compartment. After $24 \mathrm{~h}$, the apical uptake rate (A) and the consequent basolateral flux (B \& C) were quantified. Flux was significantly higher for cholesterol than for the plant sterols. Campesterol flux was significantly higher than sitosterol. Values are represented as mean (\%) of 6 independent experiments $+/-$ SD. $* *: \mathrm{P}<0.01$ and $* * *: \mathrm{P}<0.001$ 
Table I: $6 \mathrm{~h}$ and $24 \mathrm{~h}$ uptake and $6 \mathrm{~h}$ during efflux upon $24 \mathrm{~h}$ uptake of d6-sitosterol, d6-campesterol and d6-cholesterol in a neuroblastoma, astrocytoma and oligodendro-glioma cell line.

\begin{tabular}{|c|c|c|c|c|c|c|}
\hline \multirow{2}{*}{$\begin{array}{c}\text { UPTAKE } \\
(\%)\end{array}$} & \multicolumn{2}{|c|}{ d6-sitosterol } & \multicolumn{2}{|c|}{ d6-campesterol } & \multicolumn{2}{|c|}{ d6-cholesterol } \\
\hline & $6 \mathrm{~h}$ & $24 \mathrm{~h}$ & $6 \mathrm{~h}$ & $24 h$ & $6 \mathrm{~h}$ & $24 \mathrm{~h}$ \\
\hline neuron. & $4.2(0.6)$ & $15.5(1.0)$ & $4.4(0.6)$ & $15.5(0.9)$ & $7.9(1.5)$ & $20.4(1.9)$ \\
\hline astrocyt. & $11.1(0.6)$ & $42.1(5.7)$ & $10.2(0.6)$ & $44.1(3.5)$ & $10.1(0.5)$ & $44.5(3.6)$ \\
\hline oligod. & $9.8(0.8)$ & $34.4(3.0)$ & $10.4(0.7)$ & $34.2(2.7)$ & $15.7(1.9)$ & $37.5(6.5)$ \\
\hline \multirow{2}{*}{$\begin{array}{c}\text { EFFLUX } \\
(\%)\end{array}$} & \multicolumn{2}{|c|}{ d6-sititosterol } & \multicolumn{2}{|c|}{ d6-campesterol } & \multicolumn{2}{|c|}{ d6-cholesterol } \\
\hline & $\varnothing$ & HDL & $\varnothing$ & HDL & $\varnothing$ & HDL \\
\hline neuron. & $16.7(0.4)$ & $16.8(2.3)$ & $15.9(0.4)$ & $16.7(2.1)$ & $5.5(0.4)$ & $6.81(0.7)$ \\
\hline astrocyt. & $13.2(4.1)$ & $37.3(2.9)^{*}$ & $12.7(3.4)$ & $34.7(2.9)^{\star}$ & $5.4(1.4)$ & $26.5(0.6)^{\star}$ \\
\hline oligod. & $4.2(0.7)$ & $5.7(1.4)^{*}$ & $4.1(0.7)$ & $5.6(1.4)^{\star}$ & $2.6(0.2)$ & $5.3(0.5)^{\star *}$ \\
\hline
\end{tabular}

Values are displayed in $\%+/-S D . \varnothing$ represents medium with $0.2 \%$ BSA as control acceptor. ${ }^{*}=P<0.05 ; * *=P<0.01$ Mann-Withney $U$ test $H D L$ vs $\varnothing$.

Sitosterol reduces maturation of amyloid precursor protein (APP) and consequently the production of $A \beta$

Based on the known role of cholesterol on the interaction between the amyloidogenic secretase complexes and APP within the membrane DRMs [38], N2A cells stably overexpressing APP bearing the Swedish mutation (APPswe) were incubated for $24 \mathrm{~h}$ with $10 \mu \mathrm{M}$ purified plant sterols (sitosterol, campesterol and stigmasterol) and compared with incubation with $10 \mu \mathrm{M}$ cholesterol and vehicle control (1:1000 ethanol) for APP maturation. Western blot analysis revealed an intracellular reduction in APP glycosylation upon sitosterol stimulation, whereas other sterols did not influence the APP maturation (Fig 5A). In parallel, the subsequent cleavage and secretion of APP into sAPP, A $\beta 40$ and A $\beta 42$ were significantly and consistently reduced upon sitosterol stimulation (Fig5A\&B). Except for a small increased $A \beta 40$ secretion upon stigmasterol and campesterol stimulation, $A \beta 40, A \beta 42$ and sAPP remained unmodified. Intracellular $A \beta 40$ and A $\beta 42$ levels remained unaffected under each condition compared to the vehicle (data not shown). Experiments were repeated in a SHSY5Y human neuroblastoma cell line natively expressing $\mathrm{APP}_{695}$ and HEK293 cells stably overexpressing APPswe. Again, upon sitosterol stimulation, a reduced amount of glycosylated APP, a decreased A 42 and SAPP was found in SHSY5Y cells, whereas this could

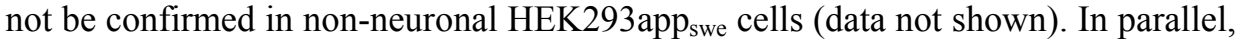
APP mRNA expression in SHSY5Y cells was significantly increased upon 
sitosterol and campesterol stimulation, but unaffected after stimulation with other sterols compared to vehicle (data not shown). Together these data show that the plant sterol sitosterol significantly reduced $A \beta 40$ and $A \beta 42$ secretion in different neuronal cell lines by lowering APP maturation.

A

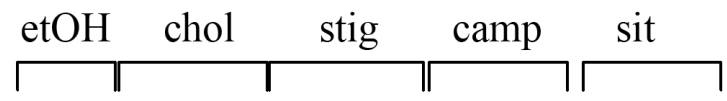

SAPP

$\rightarrow r a r$

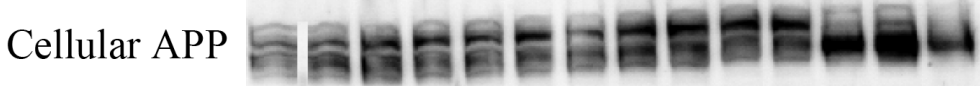

B

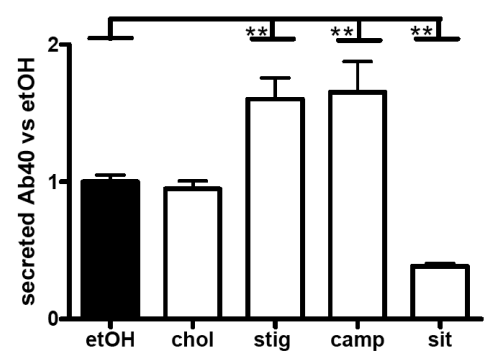

$\mathrm{C}$

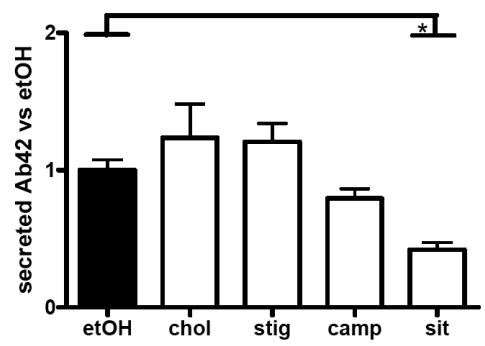

Figure 5. Sitosterol reduces APP glycosylation and subsequent APP cleavage in N2Aapp swe cells

$\mathrm{N} 2 \mathrm{Aapp}_{\text {swe }}$ cells were stimulated with $10 \mu \mathrm{M}$ sterols $(\mathrm{chol}=$ cholesterol, sit $=$ sitosterol, stig $=$ stigmasterol, camp is campesterol and etOH $=$ ethanol) for $24 \mathrm{~h}$. APP and sAPP were detected by western blot in respectively cell lysates and supernatant (A). Secreted A 340 (B) and A $\beta 42$ (C) were detected by ELISA. ANOVA with Dunnett's post hoc comparison to ethanol indicates differences as: *: $\mathrm{P}<0.05$ and ${ }^{* *}: \mathrm{P}<0.01$.

\section{Discussion}

Here we demonstrate for the first time that increased dietary intake of plant sterols results in a concentration dependent irreversible accumulation of plant sterols within the brain. In addition, we showed both in vitro and in vivo that plant sterols preferentially accumulate within DRMs of brain cells. Only upon sitosterol 
stimulation, APP cleavage into $A \beta 40$ and $A \beta 42$ peptides was significantly lowered in neuronal cell lines due to a diminished APP glycosylation.

Previously, we showed that dietary plant sterols can accumulate in the brain [36, 37]. This is confirmed by our present data showing that cerebral plant sterol concentrations significantly increased after 6 weeks dietary plant sterol supplementation, with a selective advantage for campesterol. More importantly, while plasma and liver were almost depleted from plant sterols for a period of 6 months following the 6 wks plant sterol supplementation, plant sterol concentrations in the brain remained unaffected, indicating an almost irreversible accumulation of the plant sterols. Campesterol accumulated more efficiently (1.2fold) in the brain in vivo, and also in vitro campesterol transfer across the hMEC/d3 monolayer was 1.4 times more efficient than for sitosterol. The two-fold increase in resecretion of sterols in the presence of primary astrocytes at the bottom of the lower chamber might involve the secretion of sterol accepting lipoprotein like particles by the astrocytes. Therefore, it can be assumed that both endothelial cells and astrocytes are involved in plant sterol transport across the BBB. Compared to the plant sterols we found five-fold higher re-secretion of the apical internalized cholesterol after 24h incubation. Therefore, trans-endothelial flux efficiency seems to be determined by the molecular complexity of the sterol side-chain. A possible explanation for this may be a difference in esterification rate within the endothelial cells. Similar to acyl-CoA:cholesterol O-acyltransferase (ACAT)2, mainly expressed in enterocytes, it has also been shown that ACAT1, ubiquitously expressed, esterifies cholesterol more efficient than sitosterol, independent of ACAT1 protein expression [39, 40]. However, the rate of free cholesterol in hMEC/D3 cells was more than $99 \%$, making it hard to reliably detect differences in esterification between cholesterol and plant sterols (data not shown). In general, it is assumed that the brain synthesizes all of the required cholesterol in situ and is therefore independent of peripheral cholesterol supply [11, 41]. However, several in vitro and in vivo studies demonstrated a small, but significant transport of cholesterol across the BBB. Administration 4- ${ }^{14}$-cholesterol to seven terminally ill patients revealed an average cholesterol accumulation of $3.2 \%$ cholesterol into the brain [42]. However, a defective BBB in these terminally ill patients could not be excluded. Administration of d6-cholesterol to an adult guinea pig, resulted in a $1.23 \%$ and $0.93 \%$ accumulation in respectively cerebrum and cerebellum [43]. In addition, feeding of $0.5 \% \mathrm{~d} 6$-cholesterol to mice and rats for a period of 10 days resulted in both species into a cerebral accumulation of less than 1\% [44]. An in vitro porcine endothelial brain cells Transwell ${ }^{\circledR}$ setup revealed an about $2 \%$ apical flux of cholesterol after basolateral administration [45]. In our experiments, the 
basolateral uptake of both plant sterols and cholesterol over $24 \mathrm{~h}$ is very low (campesterol: $0.26 \%$, sitosterol: $0.21 \%$ and cholesterol $3.68 \%$ compared to the common $52 \%$ uptake at the apical side; data not shown). The subsequent apical secretion was only very a very limited percentage of the absorbed sterols (campesterol: $4.74 \%$ sitosterol: $1.83 \%$ and cholesterol: $2.32 \%$; data not shown), making the absolute flux more efficient from apical to basolateral side than the reverse transport. Together, these data all show a small but significant flux of cholesterol across the BBB. However, due to the high cerebral cholesterol content and due to the tightly regulated cholesterol turnover mechanisms, this marginal amount of cholesterol crossing the BBB is expected to be negligible and to be balanced by cerebral cholesterol homeostatic mechanims. In the human brain, cholesterol is metabolized into the brain-specific 24(S)-hydroxycholesterol in order facilitate cerebral cholesterol efflux, accounting for $60 \%$ of cholesterol efflux [4648]. Plant sterols cross the endothelial monolayer even less efficiently than cholesterol. Yet, plant sterols cannot be turned into 24(S)-hydroxysterols due to the methyl/ethyl group on this C-24 position. This might, at least partly, explain why plant sterols, once crossed the BBB, mixing within this large cholesterol pool, can hardly leave the brain, leading to the almost irreversible accumulation as demonstrated in this study.

Our in vitro endothelium Transwell ${ }^{\circledR}$ system shows a rather slow transport of plant sterols through endothelial cells of the vessel walls. It needs to be taken into account that plant sterol accumulation in endothelial cells can lead to impaired endothelium-dependent vasorelaxation and increased cerebral lesion size after middle cerebral artery occlusion followed by reperfusion [9, 49]. Behind the BBB, we demonstrated that plant sterols are residing in the lipid rafts within the brain cell membranes. Moreover, plant sterols reduce the molecular order in membranes by interacting less efficiently with saturated phospholipids compared to cholesterol as required in the formation of compact, liquid ordered lipid rafts and therefore alter the membrane fluidity $[50,51]$. It was demonstrated that the magnitude of the foregoing effect depends on the geometry of the sterol molecule that is determined by the structure of its side chain (cholesterol $>>$ campesterol $>$ sitosterol $>$ stigmasterol) [50]. Mild membrane cholesterol reduction impacts the cleavage of APP upstream of $\gamma$-secretase and is mediated by changes in APP trafficking and partitioning into lipid rafts [17]. Therefore, we hypothesized that incorporation of plant sterols in lipid rafts would disturb lipid raft resident $\gamma$ secretase cleavage of APP. However, our data indicate that only sitosterol and not campesterol or stigmasterol reduced the glycosylation of APP. Therefore, it is unlikely that the altered membrane fluidity due to plant sterol incorporation is involved in the 
reduced secretion of $A \beta 40 / 42$ upon sitosterol stimulation [52]. Our findings were confirmed in a human neuronal cell line endogenously expressing $\mathrm{APP}_{695}$, whereas this could not be confirmed in non-neuronal HEK cells, stably overexpressing $\mathrm{APP}_{\text {swe }}$, suggesting it is a neuron-specific effect.

So far, consumption of plant sterol fortified diets on neurocognitive functioning or mood in non-demented statin-treated hypercholesterolemic individuals could not be detected [53]. Recently we demonstrated in a case-control study that AD patients display an impaired cholesterol turnover $[54,55]$. Liver X receptors (LXRs) are so called "master regulators" of cholesterol turnover [56]. We showed that cerebral LXR activation with the synthetic compound T0901317 resulted in an improved cerebral cholesterol turnover associated with significantly improved memory functions in our APPSLXPS1mut mouse model [57]. However, control mice being fed the LXR agonist did not further improve memory performance, stressing the specific AD modulating influence of LXR activation. Plant sterols have been found to activate LXRs $[58,59]$. Therefore, prospective studies are required on the influence of long term intake of plant sterol fortified foods on the development of $\mathrm{AD}$ and cognitive consequences.

\section{Acknowledgements}

The project was financially supported by Marie Curie Early Stage Training Fellowship (MEST-CT-2005-02058) and by funding from the European Community's Seventh Framework Programme (FP7/2007-2013) under grant agreement $\mathrm{n}^{\circ} 211696$. All experimental procedures concerning the used mice were performed according to German governmental guidelines. There are no actual conflicts of interest. 


\section{References}

1. Pollak, O.J. and D. Kritchevsky, Sitosterol. Monogr Atheroscler, 1981. 10: p. 1-219.

2. Salen, G., E.H. Ahrens, Jr., and S.M. Grundy, Metabolism of beta-sitosterol in man. J Clin Invest, 1970. 49(5): p. 952-67.

3. Law, M., Plant sterol and stanol margarines and health. Bmj, 2000. 320(7238): p. 861-4.

4. Lees, A.M., et al., Plant sterols as cholesterol-lowering agents: clinical trials in patients with hypercholesterolemia and studies of sterol balance. Atherosclerosis, 1977. 28(3): p. 325-38.

5. Thompson, G.R. and S.M. Grundy, History and development of plant sterol and stanol esters for cholesterol-lowering purposes. Am J Cardiol, 2005. 96(1A): p. 3D-9D.

6. $\quad$ Ostlund, R.E., Jr., Phytosterols and cholesterol metabolism. Curr Opin Lipidol, 2004. 15(1): p. 3741.

7. Clifton, P., Lowering cholesterol - a review on the role of plant sterols. Aust Fam Physician, 2009. 38(4): p. 218-21.

8. Calpe-Berdiel, L., J.C. Escola-Gil, and F. Blanco-Vaca, New insights into the molecular actions of plant sterols and stanols in cholesterol metabolism. Atherosclerosis, 2008.

9. Weingartner, O., et al., Vascular effects of diet supplementation with plant sterols. J Am Coll Cardiol, 2008. 51(16): p. 1553-61.

10. Calpe-Berdiel, L., et al., Increased plasma levels of plant sterols and atherosclerosis: a controversial issue. Curr Atheroscler Rep, 2009. 11(5): p. 391-8.

11. Turley, S.D., et al., Brain does not utilize low density lipoprotein-cholesterol during fetal and neonatal development in the sheep. J Lipid Res, 1996. 37(9): p. 1953-61.

12. Dietschy, J.M. and S.D. Turley, Cholesterol metabolism in the brain. Curr Opin Lipidol, 2001. 12(2): p. 105-12.

13. Yu, L., et al., Disruption of Abcg5 and Abcg8 in mice reveals their crucial role in biliary cholesterol secretion. Proc Natl Acad Sci U S A, 2002. 99(25): p. 16237-42.

14. Mulder, M., Sterols in the central nervous system. Curr Opin Clin Nutr Metab Care, 2009. 12(2): p. $152-8$.

15. Shobab, L.A., G.Y. Hsiung, and H.H. Feldman, Cholesterol in Alzheimer's disease. Lancet Neurol, 2005. 4(12): p. 841-52.

16. Stefani, M. and G. Liguri, Cholesterol in Alzheimer's disease: unresolved questions. Curr Alzheimer Res, 2009. 6(1): p. 15-29.

17. Guardia-Laguarta, C., et al., Mild cholesterol depletion reduces amyloid-beta production by impairing APP trafficking to the cell surface. J Neurochem, 2009. 110(1): p. 220-30.

18. Ledesma, M.D. and C.G. Dotti, Amyloid excess in Alzheimer's disease: what is cholesterol to be blamed for? FEBS Lett, 2006. 580(23): p. 5525-32.

19. Ehehalt, R., et al., Amyloidogenic processing of the Alzheimer beta-amyloid precursor protein depends on lipid rafts. J Cell Biol, 2003. 160(1): p. 113-23.

20. Martin, S.W., B.J. Glover, and J.M. Davies, Lipid microdomains--plant membranes get organized. Trends Plant Sci, 2005. 10(6): p. 263-5.

21. Mongrand, S., et al., Lipid rafts in higher plant cells: purification and characterization of Triton X100-insoluble microdomains from tobacco plasma membrane. J Biol Chem, 2004. 279(35): p. 36277-86.

22. Rebolj, K., et al., Steroid structural requirements for interaction of ostreolysin, a lipid-raft binding cytolysin, with lipid monolayers and bilayers. Biochim Biophys Acta, 2006. 1758(10): p. 1662-70.

23. Weksler, B.B., et al., Blood-brain barrier-specific properties of a human adult brain endothelial cell line. Faseb J, 2005. 19(13): p. 1872-4.

24. Richter-Landsberg, C. and M. Heinrich, OLN-93: a new permanent oligodendroglia cell line derived from primary rat brain glial cultures. J Neurosci Res, 1996. 45(2): p. 161-73.

25. Tamboli, I.Y., et al., Inhibition of glycosphingolipid biosynthesis reduces secretion of the betaamyloid precursor protein and amyloid beta-peptide. J Biol Chem, 2005. 280(30): p. 28110-7.

26. Walter, J., et al., Ectodomain phosphorylation of beta-amyloid precursor protein at two distinct cellular locations. J Biol Chem, 1997. 272(3): p. 1896-903.

27. Reijerkerk, A., et al., Diapedesis of monocytes is associated with MMP-mediated occludin disappearance in brain endothelial cells. Faseb J, 2006. 20(14): p. 2550-2.

28. Dixon, W.J., Analyses of extreme values. Annals of Mathematical Statistics, 1959. 21: p. 488-506. 
29. Dixon, W.J., Ratios involving extreme values. Annals of Mathematical Statistics, 1959. 22: p. 6878.

30. Prat, A., et al., Glial cell influence on the human blood-brain barrier. Glia, 2001. 36(2): p. 145-55.

31. Redgrave, T.G., D.C. Roberts, and C.E. West, Separation of plasma lipoproteins by densitygradient ultracentrifugation. Anal Biochem, 1975. 65(1-2): p. 42-9.

32. Yoon, I.S., et al., Low-density lipoprotein receptor-related protein promotes amyloid precursor protein trafficking to lipid rafts in the endocytic pathway. Faseb J, 2007. 21(11): p. 2742-52.

33. Nebl, T., et al., Proteomic analysis of a detergent-resistant membrane skeleton from neutrophil plasma membranes. J Biol Chem, 2002. 277(45): p. 43399-409.

34. Lutjohann, D., et al., Profile of cholesterol-related sterols in aged amyloid precursor protein transgenic mouse brain. J Lipid Res, 2002. 43(7): p. 1078-85.

35. Thelen, K.M., et al., Brain cholesterol synthesis in mice is affected by high dose of simvastatin but not of pravastatin. J Pharmacol Exp Ther, 2006. 316(3): p. 1146-52.

36. Jansen, P.J., et al., Dietary plant sterols accumulate in the brain. Biochim Biophys Acta, 2006.

37. Fricke, C.B., et al., Increased plant sterol and stanol levels in brain of Watanabe rabbits fed rapeseed oil derived plant sterol or stanol esters. Br J Nutr, 2007. 98(5): p. 890-9.

38. Vetrivel, K.S. and G. Thinakaran, Membrane rafts in Alzheimer's disease beta-amyloid production. Biochim Biophys Acta, 2010.

39. Liu, J., et al., Investigating the allosterism of acyl-CoA:cholesterol acyltransferase (ACAT) by using various sterols: in vitro and intact cell studies. Biochem J, 2005. 391(Pt 2): p. 389-97.

40. Temel, R.E., et al., Compared with Acyl-CoA:cholesterol O-acyltransferase (ACAT) 1 and lecithin:cholesterol acyltransferase, ACAT2 displays the greatest capacity to differentiate cholesterol from sitosterol. J Biol Chem, 2003. 278(48): p. 47594-601.

41. Dietschy, J.M. and S.D. Turley, Control of cholesterol turnover in the mouse. J Biol Chem, 2002. 277(6): p. 3801-4.

42. Chobanian, A.V. and W. Hollander, Body cholesterol metabolism in man. I. The equilibration of serum and tissue cholesterol. J Clin Invest, 1962. 41: p. 1732-7.

43. Lutjohann, D., et al., High doses of simvastatin, pravastatin, and cholesterol reduce brain cholesterol synthesis in guinea pigs. Steroids, 2004. 69(6): p. 431-8.

44. Meaney, S., et al., Formation of oxysterols from different pools of cholesterol as studied by stable isotope technique: cerebral origin of most circulating 24S-hydroxycholesterol in rats, but not in mice. Biochim Biophys Acta, 2000. 1486(2-3): p. 293-8.

45. Panzenboeck, U., et al., ABCA1 and scavenger receptor class B, type I, are modulators of reverse sterol transport at an in vitro blood-brain barrier constituted of porcine brain capillary endothelial cells. J Biol Chem, 2002. 277(45): p. 42781-9.

46. Xie, C., et al., Quantitation of two pathways for cholesterol excretion from the brain in normal mice and mice with neurodegeneration. J Lipid Res, 2003. 44(9): p. 1780-9.

47. Bjorkhem, I., et al., Cholesterol homeostasis in human brain: turnover of 24S-hydroxycholesterol and evidence for a cerebral origin of most of this oxysterol in the circulation. J Lipid Res, 1998. 39(8): p. 1594-600.

48. Lund, E.G., et al., Knockout of the cholesterol 24-hydroxylase gene in mice reveals a brainspecific mechanism of cholesterol turnover. J Biol Chem, 2003. 278(25): p. 22980-8.

49. Hodzic, A., et al., Differential modulation of membrane structure and fluctuations by plant sterols and cholesterol. Biophys J, 2008. 94(10): p. 3935-44.

50. Hac-Wydro, K., et al., Cholesterol and phytosterols effect on sphingomyelin/phosphatidylcholine model membranes--thermodynamic analysis of the interactions in ternary monolayers. J Colloid Interface Sci, 2009. 329(2): p. 265-72.

51. $\mathrm{Xu}, \mathrm{X}$., et al., Effect of the structure of natural sterols and sphingolipids on the formation of ordered sphingolipid/sterol domains (rafts). Comparison of cholesterol to plant, fungal, and disease-associated sterols and comparison of sphingomyelin, cerebrosides, and ceramide. J Biol Chem, 2001. 276(36): p. 33540-6.

52. Galbete, J.L., et al., Cholesterol decreases secretion of the secreted form of amyloid precursor protein by interfering with glycosylation in the protein secretory pathway. Biochem J, 2000. 348 Pt 2: p. 307-13.

53. Schiepers, O.J., et al., Consuming Functional Foods Enriched with Plant Sterol or Stanol Esters for 85 Weeks Does Not Affect Neurocognitive Functioning or Mood in Statin-Treated Hypercholesterolemic Individuals. J Nutr, 2009.

54. Kolsch, H., et al., Alterations of cholesterol precursor levels in Alzheimer's disease. Biochim Biophys Acta, 2010. 
55. Kolsch, H., et al., Altered levels of plasma 24S- and 27-hydroxycholesterol in demented patients. Neurosci Lett, 2004. 368(3): p. 303-8.

56. Wojcicka, G., et al., Liver X receptors (LXRs). Part I: structure, function, regulation of activity, and role in lipid metabolism. Postepy Hig Med Dosw (Online), 2007. 61: p. 736-59.

57. Vanmierlo, T., et al., Liver $X$ receptor activation restores memory in aged AD mice without reducing amyloid. Neurobiol Aging, 2009.

58. Plat, J., J.A. Nichols, and R.P. Mensink, Plant sterols and stanols: effects on mixed micellar composition and LXR (target gene) activation. J Lipid Res, 2005. 46(11): p. 2468-76.

59. Yang, C., et al., Sterol intermediates from cholesterol biosynthetic pathway as liver $X$ receptor ligands. J Biol Chem, 2006. 281(38): p. 27816-26. 



\section{Chapter V}

\section{A Study on the Role of Cerebral Plant Sterol Accumulation on Memory and Anxiety Related Behavior in Abcg5-/- mice}




\section{Chapter V}

\section{A Study on the Role of Cerebral Plant Sterol Accumulation on Memory and Anxiety Related Behavior in Abcg5-/- mice}

Tim Vanmierlo, a,b, Kris Rutten,, Leonie C. van Vark -van der Zee ${ }^{\mathrm{c}}$, Silvia Friedrichs ${ }^{\mathrm{b}}$, Vincent W. Bloks ${ }^{\mathrm{d}}$, Arjan Blokland ${ }^{\mathrm{e}, \mathrm{g}}$, Frans C. Ramaekers ${ }^{\mathrm{f}}$, Jos Prickaerts $^{\mathrm{a}, \mathrm{g}}$, Harry Steinbusch ${ }^{\mathrm{a}, \mathrm{g}}$, Folkert Kuipers ${ }^{\mathrm{d}}$, Dieter Lütjohann ${ }^{\mathrm{b}, \mathrm{g}}$, Monique Mulder ${ }^{\mathrm{a}, \mathrm{g}}$

a Department of Neuroscience, Maastricht University, Maastricht, The Netherlands; ${ }^{\mathrm{b}}$ Institute of Clinical Chemistry and Pharmacology, University Clinics Bonn, Bonn, Germany; ${ }^{\mathrm{c}}$ Department of Internal Medicine, Division of Pharmacology, Vascular and Metabolic Diseases, Erasmus Medical Center, Rotterdam, The Netherlands; ${ }^{\mathrm{d}}$ Department of Pediatrics, University Medical Center Groningen, Groningen, The Netherlands; ${ }^{\mathrm{e}}$ Department of Neuropsychology and Psychopharmacology, Maastricht University, Maastricht, The Netherlands; ${ }^{\mathrm{f}}$ Department of Molecular Cell Biology, Maastricht University, Maastricht, The Netherlands; ${ }^{\mathrm{g}}$ EURON (European Graduate School for Neuroscience) 


\section{Abstract}

Plant sterols such as sitosterol and campesterol are frequently applied as functional food additives in the prevention of atherosclerosis. Recently, it became clear that plasma derived plant sterols accumulate in murine brains.

We addressed the question whether plant sterol accumulation in the brain is associated with alterations in brain cholesterol homeostasis and subsequently its functions, using Abcg5-/- mice, a model for phytosterolemia. In Abcg5-/- mice on a standard chow diet serum and brain plant sterol concentrations were increased 3570-fold and 5-12-fold, respectively. Compared with wild type mice, Abcg5-/- mice accumulated campesterol in the brain, particularly in the cerebellum, despite raise sitosterol levels in the serum. In addition, lanosterol concentrations were increased in the cortex, whereas desmosterol and 24(S)-hydroxycholesterol were decreased in the hippocampus. Abcg5-/- mice challenged with a diet high in plant sterols resulted in modestly increased expression of Liver X receptor target genes Abcg1 and Abca1 in their brains. Abcg5-/- and Abcg5+/+ mice fed a standard chow diet, showed no differences in learning and memory functions or in anxiety and mood related behavior, as assessed by performance in behavioral tasks. An exception was that Abcg5-/- mice swam faster than Abcg5+/+ mice in the Morris Water Maze task.

In conclusion, accumulation of plant sterols in the brains of Abcg5-/- mice does have limited consequences for brain cholesterol metabolism, but does not lead to an overt phenotype in either learning and memory functions or mood and anxiety related behavior in the behavioral tasks applied.

\section{Introduction}

Consumption of high amounts of plant sterols leads to reduced serum and LDL cholesterol concentrations, making them attractive compounds in the treatment of atherosclerosis and cardiovascular diseases (CVD) [1]. As such, they are frequently used as functional food additives in the prevention of atherosclerosis, although no hard end-point studies are available [2,3]. Plant sterols, exclusively derived from the diet, differ from cholesterol only by an additional ethyl or methyl group at $\mathrm{C} 24$ and/or a double bond at C22 [4,5]. All cholesterol within the brain is synthesized in situ, since circulating cholesterol is prevented from entering the brain by the bloodbrain barrier (BBB). Unlike dietary cholesterol, however, plant sterols can accumulate in the brain $[6,7]$. 
ATP binding cassette G5 (ABCG5) and ABCG8 act as functional heterodimer transporters at the apical membranes of enterocytes and hepatocytes where they resecrete plant sterols into the intestinal lumen and bile, respectively [8]. Despite this exclusion mechanism, small amounts of plant sterols reach the brain. In humans and mice defects in ABCG5 and/or ABCG8 cause phytosterolemia, a rare autosomal recessive disorder characterized by a massive plant sterol accumulation in the circulation and tissues [9-11]. Patients with phytosterolemia display xanthoma, an increased risk of premature atherosclerosis, hemolysis, and macrothrombocytopenia [12-16].

Brain cholesterol homeostasis is a complex but well orchestrated system. Disturbances of this system are associated with severe neurological diseases, such as Niemann-Pick disease type $\mathrm{C}$ and Smith-Lemli-Opitz's syndrome [17, 18]. Plant sterols may alter brain cholesterol metabolism by enhancing cholesterol turnover through activation of Liver X receptors (LXRs) [19, 20]. LXR-activation was found to improve memory and brain cholesterol turnover in a model of Alzheimer's Disease [21].

Besides an involvement of brain cholesterol metabolism in memory processes [22, 23], patients with depression, anxiety, co-morbid depression, suicidal ideation and current or past suicidal behavior display low serum cholesterol levels $(<160 \mathrm{mg} / \mathrm{dl})$ (for review, see: [24]). Monkeys fed a low cholesterol diet exhibited less affiliative interaction in comparison with their counterparts fed a normal diet [25]. Part of the mechanism for this may be linked with the in vitro finding that decreased neuronal membrane cholesterol concentrations were shown to reduce serotonin neurotransmission [26-28]. Phytosterolemia patients which are rare $( \pm 100$ known cases worldwide), notably have relatively high educational levels (personal communication Dr. G. Salen). Therefore, we addressed the question whether accumulation of plant sterols in the brain interferes with brain cholesterol homeostasis and if this consequently affects learning, memory, mood, and anxiety related behavior.

\section{Materials and Methods}

Animals

Abcg5-/- mice and Abcg5+/+ littermates were generated by intercrossing F1 heterozygotes on a mixed C57BL/6Jx129/OlaHsd background by Deltagen (Redwood City, CA, USA) as described by Plösch et al. [29]. In parallel to the 
human situation, in which the plant sterol fortified food consumption starts at an adult age, mice were fed either a standard laboratory chow diet $(\mathrm{n}(\mathrm{Abcg}+/+)=9$ and $\mathrm{n}(\mathrm{Abcg} 5-/-)=9)$ or a high plant sterol containing diet $(\mathrm{n}(\mathrm{Abcg}+/+)=6$ and $\mathrm{n}($ Abcg5-/-) $=6)(0.5 \% \mathrm{wt} / \mathrm{wt} ; 65 \%$ sitosterol and 35\% campesterol) starting from the adult age of 4 months, for 3 months. All animals had ad libitum access to the appropriate diet and water. One week prior to the behavioral experiments, mice were housed singly in individually ventilated cages in the room where the behavioural tests were performed. However, mice on a high plant sterol diet were not included in the behavioral experiments due to the high mortality rate $(50 \%)$ in the Abcg5-/- group (Kaplan- Meier survival analysis (Abcg5+/+: t1: $\mathrm{n}=6$; $\mathrm{t} 2: \mathrm{n}=6$; and Abcg5-/-: t1: $\left.\mathrm{n}=6 ; \mathrm{t} 2: \mathrm{n}=3): \chi^{2}(1)=3.67 ; \mathrm{P}=0.05\right)$. Therefore, preceding behavioral experiments, these mice were sacrificed at an age of 7 months. Mice on the normal diet $(\mathrm{n}(\mathrm{Abcg}+/+)=9$ and $\mathrm{n}(\mathrm{Abcg} 5-/-)=8)$ were kept on a regular $12 \mathrm{~h}$ day/night cycle (lights on 7AM; lights off 7PM) and were subjected to a battery of behavioral tasks between 7 and 9 months. A radio played softly, providing background noise in the room. Testing always took place between $8 \mathrm{~h} 00$ and $15 \mathrm{~h} 00$. Handling procedures were conform previous descriptions [30]. Tests were performed by an experimenter, unaware of the genetic background of the animals. The sequence of behavioral tasks was set to perform the least stressful tasks first, and the more stressful tasks last. Therefore, the object recognition task was performed first, followed by open field task, elevated zero maze, Morris water escape maze and finally the tail suspension task. Each task was separated by three resting days, trying to reduce the interference between the tasks to a minimum. The code was broken after mice were sacrificed at the age of 9 months. All experimental procedures were approved by the local ethical committee of Maastricht University (The Netherlands) for animal experiments according to governmental guidelines.

\section{Object Recognition Task}

The object recognition task (ORT) was performed as described previously [30, 31]. In brief, the apparatus consisted of a circular arena of $43 \mathrm{~cm}$ in diameter. Half of the $40 \mathrm{~cm}$ high wall consisted of white polyvinyl chloride, the other half of transparent polyvinyl chloride. The different objects were: (1) a cone made of brass (maximal diameter $6 \mathrm{~cm}$ and total height $3.8 \mathrm{~cm}$ ), (2) a transparent glass bottle (diameter 2.7 $\mathrm{cm}$, height $8.5 \mathrm{~cm})$ filled with sand, (3) a heavy metal cube $(2.5 \mathrm{~cm} \times 5 \mathrm{~cm} \times 7.5$ $\mathrm{cm}$ ) with two holes (diameter $1.5 \mathrm{~cm}$ ), and (4) a heavy aluminium cube with a tapering top $(4.5 \mathrm{~cm} \times 4.5 \mathrm{~cm} \times 8.5 \mathrm{~cm})$. None of the objects could be moved by the mice. The order of objects used per subject per session was determined using a randomization scheme. For habituation purposes, mice were allowed to explore the empty arena in trials of $4 \mathrm{~min}$ for two days. On the third day of the habituation, 
mice were subjected to two trials of 4 minutes in the arena with two similar objects. The two objects were placed symmetrically about $10 \mathrm{~cm}$ away from the wall of the circular arena. After one resting day, the experiment started at 50 lux illumination. During the first trial ( $\operatorname{Tr} 1)$ the apparatus contained two identical objects (samples). After the first exploration period of $4 \mathrm{~min}$, the mouse was placed back into its home cage. Subsequently, after a predetermined delay interval of $1 \mathrm{~h}$, the mouse was returned to the arena for the second trial of $4 \mathrm{~min}(\mathrm{Tr} 2)$, but now with two dissimilar objects: the familiar object (the sample) and a new object. The time spent exploring each object during $\operatorname{Tr} 1$ and $\operatorname{Tr} 2$ was recorded manually using a personal computer. Total exploration time (e.g. sum of the time spent to explore either of the objects) in Tr1 and Tr2 will respectively be referred to as T1 and T2. Sitting on the object was not considered as exploratory behavior. The discrimination index $\mathrm{d} 2$ in $\operatorname{Tr} 2$ was calculated as measure for object memory $(\mathrm{d} 2=[($ exploration time for the novel object in $\operatorname{Tr} 2$ ) - (exploration time for the familiar object in $\operatorname{Tr} 2)] / \mathrm{T} 2$ ).

\section{The Morris Water Escape Maze}

Spatial memory performance was measured in the Morris water escape maze (MWEM). The maze consisted of a circular gray water tank (diameter $81 \mathrm{~cm}$ ) filled with clear tap water $\left(22-24^{\circ} \mathrm{C}\right)$. A video camera, centrally positioned above the water tank, automatically recorded movements of mice at a rate of five samples per second via a tracking system (Etho Vision ${ }^{\mathrm{TM}}$, Noldus, Wageningen, The Netherlands). The mice were subjected to a total of 24 acquisition trials: 6 days, 4 trials a day, separated by a 5 min inter-trial interval, using 4 different starting positions assigned in a random order between mice to avoid side bias. A gray platform (diameter $5 \mathrm{~cm}$ ), submerged beneath the water surface, was located at one fix position for all mice throughout the experiments. If a mouse was unable to find the platform within $60 \mathrm{~s}$, it was guided to the platform and had to climb onto the platform. Mice were allowed to stay on the platform for $3 \mathrm{~s}$ before being returned to their home cage. During all acquisition trials, swimming speed $(\mathrm{cm} / \mathrm{s})$, distance moved $(\mathrm{cm})$, and swimming speed before reaching the platform (s) were measured (Etho Vision ${ }^{\mathrm{TM}}$, Noldus, Wageningen, The Netherlands). Next, $1 \mathrm{~h}$ after the final acquisition trial (day 6), mice were subjected to a probe trial in which the platform was removed from the pool. Mice were released into the pool, opposite the previous platform location and allowed to swim for $60 \mathrm{~s}$. The arena was divided into four quadrants, with the target zone being the quadrant in which the platform was formerly located. As depicted in figure 2e, the other quadrants were called respectively left, right and opposite. The computer assisted camera device recorded the time spent in each quadrant as described previously $[32,33]$. 


\section{Open Field}

The open field test (OF) was conducted as described previously [23]. The arena consisted of a square, clear Plexiglas box $(50 \mathrm{~cm} \times 50 \mathrm{~cm} \times 30 \mathrm{~cm})$, with an open top and a grey floor. The arena was subdivided into 'corner' (four squares each $8 \mathrm{~cm} \mathrm{x}$ $8 \mathrm{~cm}$ ), 'wall' (four rectangles each $34 \mathrm{~cm} \times 8 \mathrm{~cm}$ ) and 'centre' $(34 \mathrm{~cm} \times 34 \mathrm{~cm})$ zones. A camera was installed $2.5 \mathrm{~m}$ above the centre of the field. Immediately after a mouse was placed in the centre of the OF, the movements and position of the animals were recorded and registered automatically by a computerized system (Etho Vision $^{\mathrm{TM}}$, Noldus, Wageningen, The Netherlands) in order to determine time in zone (TIZ) and distance moved (DM) over the $20 \mathrm{~min}$ trial by at about 50 lux illumination. Odour trails were removed after each trial with $70 \%$ alcohol.

\section{Elevated Zero Maze}

The elevated zero maze (EZM), originally described in Shepherd et al. (1994), was made of black, infrared-transparent, plastic [34]. It is a modification of the elevated plus maze model for anxiety in rodents [35]. It consists of a circular runway $(50 \mathrm{~cm}$ in diameter), $6 \mathrm{~cm}$ path width, $20 \mathrm{~cm}$ above floor level) which was divided equally into two opposite open zones (OZ) and two opposite zones, enclosed with $20 \mathrm{~cm}$ high side walls (CZ) [36]. Measurements were performed under almost dark conditions for the open area (1-2lux) [37, 38]. The entrance of each $\mathrm{OZ}$ and $\mathrm{CZ}$ contains at each side respectively two outer border (BO) and two inner border (BI) areas, covering $3 \mathrm{~cm}$ of the running way each (Fig. 4c). A $3 \mathrm{~mm}$ high edge surrounded the open zones to prevent falls. Mice were placed into one of the open zones and were allowed to freely explore the maze for a period of $5 \mathrm{~min}$. Time spent and distance moved in $\mathrm{OZ}, \mathrm{BI}, \mathrm{BO}$, and $\mathrm{CZ}$ were measured via an infrared video camera connected to a video tracking system (Etho Vision ${ }^{\mathrm{TM}}$, Noldus, Wageningen, The Netherlands). The maze was cleaned with $70 \%$ alcohol after each animal trial.

\section{Tail Suspension Task}

The tail suspension task (TST) is designed to test antidepressant-like activity in mice [39]. The test is based on the fact that animals subjected to the short-term, inescapable stress of being suspended by their tail, will develop an immobile posture [40]. Four cages with closed side walls and an open front were used to test the mice in the TST (18cm of width and $30 \mathrm{~cm}$ of height). Each animal was individually hung by its tail to a hook at the ceiling of the chamber, using adhesive tape. Their movements were recorded for 5 min using a video camera device, centrally positioned in front of the cages at a rate of five samples per s via an automated tracking system (Etho Vision ${ }^{\mathrm{TM}}$, Noldus, Wageningen, The 
Netherlands). The behavioral variable "immobility" was defined as no movement or no obvious effort to escape from the hook. The cutoff value for immobility was set at less than $15 \%$ movement. Total activity versus inactivity is regarded as a measure of antidepressant-like activity of plant sterols [39].

\section{Analyses}

\section{Sample preparation}

Following the behavioral experiments, mice were anaesthetized with IP ketamine/xylazine (100 mg/kg ketamine and $5 \mathrm{mg} / \mathrm{kg}$ xylazine). Subsequently, the whole body was perfused with ice cold phosphate buffered saline (PBS) (137mM $\mathrm{NaCl} ; 2.7 \mathrm{mM} \mathrm{KCl} ; 6.5 \mathrm{mM} \mathrm{Na}_{2} \mathrm{HPO}_{4} ; 1.5 \mathrm{mM} \mathrm{KH}_{2} \mathrm{PO}_{4} ; \mathrm{pH}=7.3$ ). Blood was collected prior to perfusion by puncturing the right atrium of the heart. Blood samples were spun $10 \mathrm{~min}$ at $0.2 \mathrm{G}$ to obtain serum. Prior to the sterol analysis extraction, brains were divided in two hemispheres, then the cerebellum, whole hippocampus and top part of the neo-cortex (Bregma -0.5 to -4 ) were dissected out and snap frozen. Brain hemispheres of 2 Abcg5+/+ mice on normal diets were omitted due to wrong dissection and excluded from the sterol analysis. One hemisphere of each Abcg5+/+ and each Abcg5-/- mouse on high plant sterol diet was used for mRNA extraction. The other hemisphere was examined for pathological brain haemorrhage using immunohistochemical staining, but no reliable data were generated (data not shown).

\section{Sterol profile determination}

Samples were spun in a Speedvac (12mbar) (Savant AES 1000) for 24h so that we could relate individual sterol concentrations to dry weight. The sterols were extracted from the dried tissue by placing them in a $2 \mathrm{ml}$ mixture of chloroformmethanol $(2: 1)$ for $24 \mathrm{~h}$ at $4^{\circ} \mathrm{C}$. Sterol levels were determined by gas chromatography/mass spectrometry as described previously [41].

\section{$R N A$ isolation and RT-procedures}

Single brain hemispheres from Abcg5+/+ and Abcg5-/- mice on high plant sterol diet were homogenized in $1 \mathrm{ml}$ of Trizol Reagent (In vitrogen, Breda, The Netherlands) preceding RNA isolation. Total RNA was isolated using the Trizol Reagent method (Invitrogen, Breda, The Netherlands) according to manufacturer instructions and quantified using Ribogreen (Molecular Probes, Inc., Eugene, OR). cDNA equivalent to $10 \mathrm{ng}$ of total RNA was amplified using the Q-RT-PCR core kit (Eurogentec, Seraing, Belgium) according to the manufacturer's protocol. QRT-PCR was performed using an Applied Biosystems 7700 sequence detector according to the manufacturer's instructions, as described previously [42], with 
modifications [11]. Primer oligomers (Invitrogen, Breda, The Netherlands) and fluorogenic probes, labeled with 6-carboxyfluorescein (5'-FAM) and 6carboxytetramethylrhodamine (3'-TAMRA), were purchased from Eurogentec (Seraing, Belgium). APP primer and probe sequences were generated using ABI PRISM $^{\mathrm{TM}}$ Primer Express 2.0.0 ${ }^{\mathrm{TM}}$ (Applied Biosystems, Foster City, USA) CCGTGGCACCCTTTTGG (forward), GGGCGGGCGTCAACA (reverse) and AGCCAATACCGAAAAT (probe). Other primer and probe sequences (Dhcr24 and 18S) have been published in Vanmierlo et al. [43] or under RTprimerDB IDs (http://www.labpediatricsrug.nl latest access on 0601 2010): 524 (Hmgcr), 522 (Cyp27a1), 3597 (Cyp46a1), 516 (Abca1), 1263 (Abcg1), 493 (Apoe), 1276 (Apoc1), 496 (Ldlr), 501 (Lxra), 4239 (Lxrb), 8118 (Cyp51) and 503 (Srebp1c). All expression data were subsequently normalized for $18 \mathrm{~S}$ rRNA, which was analyzed in a separate run.

\section{Statistics}

All statistical analyses were performed using GraphPad Prism 4TM. The acquisition times, acquisition distance, and swimming speed in the MWEM were statistically analyzed using the 2-way analysis of variances assay (2-way ANOVA) with experimental day and genotype as independent variables. The MWEM probe trial (between location, within genotype), the OF location data (between location, within genotype), and the serum and brain plant sterol levels (between anatomical region, within genotype) were analyzed using the 1-way ANOVA with a post hoc Bonferroni's Multiple Comparison Test (indicated as "PH"). d2, T1 and T2 values in ORT (between genotype), distance moved and time spent at each location in OF (between genotype), immobility TST (between genotype), zones and frequencies of zone entrance in EZM (between genotype), and sterol data (between genotype), were compared using an unpaired 2 -sided Student's t-test. To test whether $\mathrm{d} 2$ values in the ORT were different from 0, a one sample Student's t-test was performed. Animals that did not reach the minimum of $5 \mathrm{~s}$ exploration in ORT in either Tr1 or $\operatorname{Tr} 2$ were excluded from further analysis (Sik et al, 2003). All data sets were tested for normality. A Gaussian distribution could not been proven in the mRNA expression data. Therefore, for mRNA expression data, the genotypes were compared using the non parametrical Mann Whitney $\mathrm{U}\left(\mathrm{MWU}, \mathrm{n}_{1}, \mathrm{n}_{2}, \mathrm{P}\right)$ test. The Kaplan Meier survival analysis was verified on significance using the log rank chi square test. Sterol correlations were calculated, using Pearson's correlation test (Pearson's $\mathrm{r}^{2}$ (number of pairs);P). Extreme values were excluded by means of Dixon's principles of exclusion of extreme values [44, 45]. Significance levels were determined on $* \mathrm{P}<0.05,{ }^{*} * \mathrm{P}<0.01$ or $* * * \mathrm{P}<0.001$. Statistical details on data which 
were not statistically significantly different according to the applied test were not displayed in the results section.

\section{Results}

Abcg5-/- mice display remarkably increased plant sterol levels in all brain regions in particular in the cerebellum.

Corroborating previous results [7, 29], Abcg5-/- mice on normal chow diet displayed strongly increased serum levels of sitosterol, campesterol, and stigmasterol (30-, 5- and 70-fold, respectively) in comparison with their wild-type littermates, whereas cholesterol concentrations were significantly decreased (2-fold) (data not shown).

The hippocampus, the cortex, and the cerebellum of the Abcg5-/- mice on standard chow diet, displayed higher concentrations of sitosterol (12-fold), campesterol (7fold), and stigmasterol (5-fold) than in Abcg5+/+ mice (see Fig 1). Both Abcg5+/+ and Abcg5-/- mice display the highest absolute concentrations of sitosterol and campesterol in the cerebellum in comparison with the cortex and the hippocampus (Sitosterol in Abcg5+/+: $\mathrm{F}(2,20)=7.07, \mathrm{P}<0.01 \mathrm{PH}$ : cerebellum versus cortex $(\mathrm{t}(12)=3.44 ; \mathrm{P}<0.01)$ and hippocampus $(\mathrm{t}(12)=3.04 ; \mathrm{P}<0.05)$; Sitosterol in Abcg5-/-: $\mathrm{F}(2,23)=9.05, \mathrm{P}<0.01 \mathrm{PH}$ : cerebellum versus cortex $(\mathrm{t}(14)=3.87 ; \mathrm{P}<0.01)$ and hippocampus $(\mathrm{t}(14)=3.46 ; \mathrm{P}<0.01)$; Campesterol in Abcg5+/+: $\mathrm{F}(2,20)=6.92$, $\mathrm{P}<0.01 \mathrm{PH}$ : cerebellum versus cortex $(\mathrm{t}(12)=3.42 ; \mathrm{P}<0.01)$ and hippocampus $(\mathrm{t}(12)=2.99 ; \mathrm{P}<0.05)$; and Campesterol in Abcg5-/-: $\mathrm{F}(2,23)=9.97, \mathrm{P}<0.01 \mathrm{PH}$ : cerebellum versus cortex $(\mathrm{t}(14)=4.02 ; \mathrm{P}<0.01)$ and hippocampus $(\mathrm{t}(14)=3.70$; $\mathrm{P}<0.01)$ ) (see Fig1a). Also the ratio of sitosterol and campesterol over cholesterol was highest in the cerebellum in Abcg5-/- mice (Sitosterol: $\mathrm{F}(2,23)=5.21 ; \mathrm{P}<0.05$ $\mathrm{PH}$ : cerebellum versus cortex $(\mathrm{t}(14)=2.83 ; \mathrm{P}<0.05)$ and hippocampus $(\mathrm{t}(14)=2.76$; $\mathrm{P}<0.05)$; and Campesterol: $\mathrm{F}(2,23)=5.85 ; \mathrm{P}<0.01 \mathrm{PH}$ : cerebellum versus cortex $(\mathrm{t}(14)=2.95 ; \mathrm{P}<0.05)$ and hippocampus $(\mathrm{t}(14)=2.98 ; \mathrm{P}<0.05))$. In contrast to the Abcg5-/- mice, no brain region specific differences in plant sterol accumulation relative to local cholesterol content were found in Abcg5 $+/+$ controls. No absolute or relative differences were found for stigmasterol in either of the two groups. 


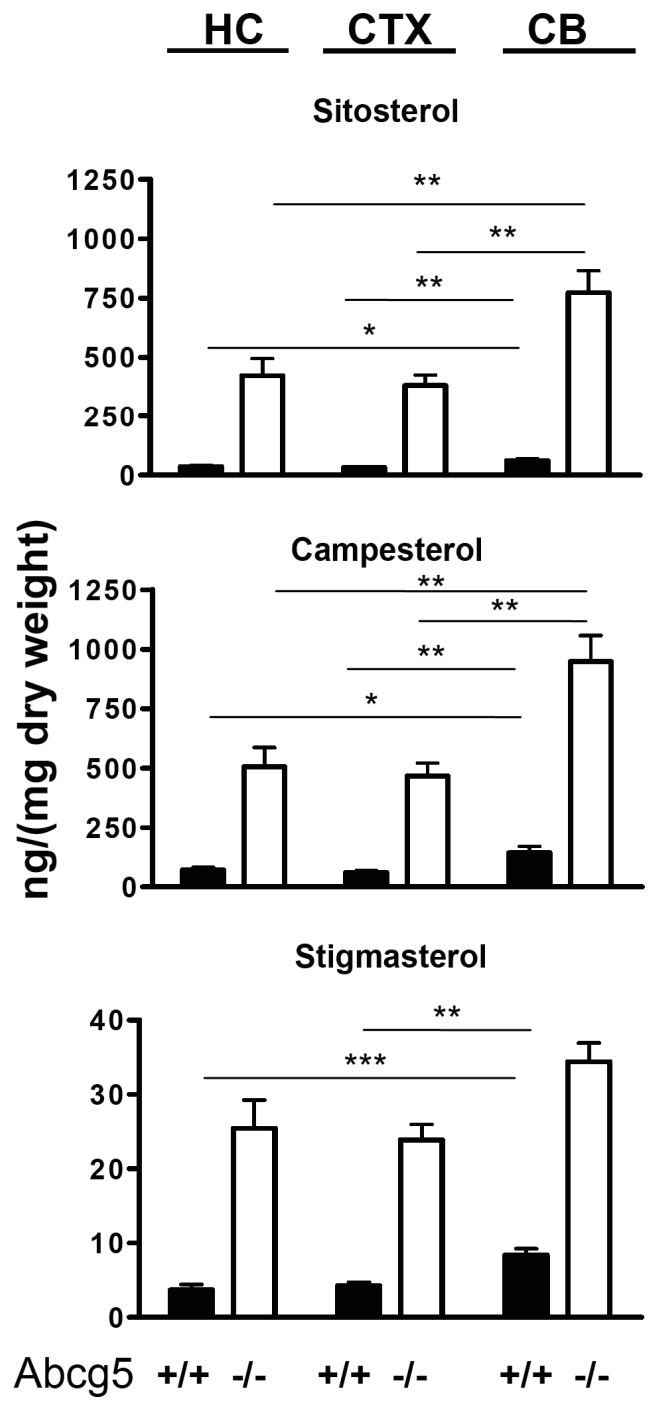

Fig.1. Plant sterol levels in specific brain regions of Abcg5+/+ and Abcg5-/- mice on normal chow diet.

Plant sterol levels in the brain were measured in three different regions: hippocampus, cortex, and cerebellum of Abcg5-/- $(n=8)$ and Abcg5+/+ $(n=7)$ mice. All plant sterols measured were markedly increased in all brain regions $(\mathrm{p}<0.001$, not indicated) of Abcg5-/- mice in comparison with Abcg5+/+ mice. Regional differences within Abcg5+/+ and Abcg5-/- mice brains are indicated: $*=\mathrm{P}<0.05, * *=\mathrm{P}<0.01, * * *=\mathrm{P}<0.001(\mathrm{~b})$. Values are displayed as mean $+/-$ SEM. 
Thus, Abcg5-/- mice on a normal diet were prone to accumulate plant sterols in their brain, in particular in the cerebellum. Moreover, in Abcg5-/- mice plant sterol levels in the brain were not directly related to their serum levels. Abcg5-/- mice displayed serum sitosterol levels that were twice as high as campesterol levels (data not shown), while campesterol and sitosterol levels were comparable in the brain. Sitosterol concentrations correlated significantly with lathosterol concentrations in Abcg5-/- cerebellum $\left(\mathrm{r}^{2}(8)=0.73 ; \mathrm{P}<0.01\right)$ and hippocampus $\left(\mathrm{r}^{2}(8)=0.51 ; \mathrm{P}<0.05\right)$, but not in the cortex or in Abcg $5+/+$ brain regions. Despite overall increases in brain plant sterol concentrations, the levels of endogenous sterols were affected to a limited extent only in specific brain regions (Table I). Lanosterol levels were increased in the cortex of Abcg5-/- mice $(\mathrm{t}(13)=3.17 ; \mathrm{P}<0.01)$ and to a lesser extent also in the hippocampus, in line with increased levels in serum $(\mathrm{t}(13)=5.18$; $\mathrm{P}<0.001)$. Desmosterol $(\mathrm{t}(13)=3.46 ; \mathrm{P}<0.01)$ and $24(\mathrm{~S})-O H c h o l e s t e r o l ~(t(12)=3.62$; $\mathrm{P}<0.01$ ) levels were significantly reduced (to $72 \%$ and $68 \%$ of the control levels, respectively) exclusively in the hippocampus.

Table I: Sterol profiles in brain regions of Abcg5-/- and Abcg5+/+ mice on normal chow diet.

\begin{tabular}{|c|c|c|c|c|c|c|}
\hline Brain & Abcg5 & HC & & CTX & & CB \\
\hline Cholesterol & $+/+$ & $71.5(3.4)$ & & $61.3(3.4)$ & & $91.2(3.6)$ \\
\hline$(\mu \mathrm{g} / \mathrm{mg})$ & $-/-$ & $66.8(5.9)$ & & $63.3(4.2)$ & & $89.0(3.6)$ \\
\hline Lathosterol & $+/+$ & $68.6(3.3)$ & & $70.0(2.1)$ & & $78.2(5.8)$ \\
\hline$(\mathrm{ng} / \mathrm{mg})$ & $-/-$ & $83.1(18.4)$ & & $88.6(9.0)$ & & $87.7(5.9)$ \\
\hline Desmosterol & $+/+$ & $422.0(14.6)$ & $* *$ & $277.9(9.0)$ & & $406.6(30.2)$ \\
\hline$(\mathrm{ng} / \mathrm{mg})$ & $-/-$ & $305.8(28.6)$ & & $312.1(19.7)$ & & $400.8(25.4)$ \\
\hline Lanosterol & $+/+$ & $13.0(2.0)$ & & $12.8(0.6)$ & $* *$ & $17.8(1.0)$ \\
\hline (ng/mg) & $-/-$ & $16.1(2.8)$ & & $17.1(1.1)$ & & $17.0(2.2)$ \\
\hline 27-OHcholesterol & $+/+$ & $6.1(2.0)$ & & $1.14(0.23)$ & & $2.68(0.5)$ \\
\hline$(\mathrm{ng} / \mathrm{mg})$ & $-/-$ & $6.7(1.3)$ & & $1.00(0.16)$ & & $2.42(0.2)$ \\
\hline 24-OHcholesterol & $+/+$ & $300.7(20.0)$ & $* *$ & $244.3(11.4)$ & & $56.61(4.3)$ \\
\hline (ng/mg) & $-/-$ & $205.8(16.5)$ & & $266.6(8.5)$ & & $48.32(3.3)$ \\
\hline
\end{tabular}

Mean \pm SEM, $*=P<0.05 \quad * *=P<0.01 \quad * * *=P<0.001$ (2 sided unpaired student's T-test). HC = hippocampus, CTX = cortex, and $C B=$ cerebellum. $n($ Abcg5+/+) $=7 \quad n($ Abcg5-/-) $=8$ 
Since we previously found no significant differences in the expression of genes involved in the brain cholesterol homeostasis of Abcg5-/- versus Abcg5+/+ mice [7], we examined gene expression in the brains of Abcg5-/- mice fed a high plant sterol diet. The expression of the LXR target genes Abca1 (MWU $=0, \mathrm{n}_{1}=6, \mathrm{n}_{2}=3$, $\mathrm{P}<0.05$ ) and Abcg1 (MWU=1, $\left.\mathrm{n}_{1}=6, \mathrm{n}_{2}=3, \mathrm{P}<0.05\right)$ were significantly increased in comparison to Abcg5+/+ mice on a high plant sterol diet (Table II). Other LXR responsive genes, Srebp1c, ApocI and LXR $\alpha$ which are known to be significantly upregulated by the synthetic LXR-activator T0901317 [21] were not affected. There was a small, but non-significant increase in Apoe mRNA. Due to the increased mortality of the Abcg5-/- mice on a high plant sterol diet, as described in the materials and methods section, no behavioral tests were performed with these mice.

Table II: mRNA expression of genes involved in brain cholesterol metabolism in brains of Abcg+/+ and Abcg5-/- mice on high plant sterol containing diet, normalized against $18 \mathrm{~S}$ rRNA expression.

\begin{tabular}{|c|c|c|c|}
\hline & Abcg5+/+ & Abcg5-/- & \\
\hline App & $1.00(0.17)$ & $1.09(0.08)$ & \\
\hline Dhrc24 & $0.99(0.07)$ & $1.00(0.13)$ & \\
\hline Cyp51 & $0.73(0.09)$ & $0.67(0.03)$ & \\
\hline Hmgcr & $0.09(0.01)$ & $0.09(0.02)$ & \\
\hline Сур27a1 & $0.05(0.01)$ & $0.03(0.00)$ & \\
\hline Сур46а1 & $0.99(0.12)$ & $1.10(0.22)$ & \\
\hline Abca1 & $0.32(0.03)$ & $0.53(0.01)$ & * \\
\hline Abcg1 & $0.93(0.08)$ & $1.85(0.36)$ & * \\
\hline Apoe & $0.77(0.09)$ & $1.04(0.06)$ & \\
\hline Apoc1 & $0.26(0.04)$ & $0.24(0.07)$ & \\
\hline Srebp1 & $0.90(0.16)$ & $1.18(0.19)$ & \\
\hline Ldlr & $0.29(0.04)$ & $0.23(0.11)$ & \\
\hline Lxra & $5.62(0.58)$ & $6.80(0.77)$ & \\
\hline Lxrb & $2.83(0.22)$ & $3.18(0.23)$ & \\
\hline
\end{tabular}

$n($ Abcg5+/+) $=6 \quad n($ Abcg5-/-) $=3$. Mean $\pm(S E M), P<0.05=*$ (non parametric MWU-test) 
No difference in learning and memory between the Abcg5-/- and Abcg5+/+ mice on normal chow diet as indicated by their performance in the ORT and the MWEM

The Abcg5-/- mice did not display any alterations in overall appearance or general behavior in comparison with the Abcg5+/+ mice. Body weights of all mice were comparable and remained stable during the testing period (data not shown).

To examine possible effects of elevated brain plant sterols on learning and memory functions, 7-month old Abcg5-/- and Abcg5+/+ littermates were subjected to the ORT and the MWEM.

There were no significant performance differences between the two groups the ORT (Fig. 2a); d2 values were significantly higher than 0 for both the Abcg5-/$(\mathrm{t}(6)=3.99 ; \mathrm{P}<0.01)$ and Abcg5+/+ mice $(\mathrm{t}(6)=5.46 ; \mathrm{P}<0.01)$, indicating the mice remembered the familiar object. The $\mathrm{d} 2$ value was slightly, but not significantly $(\mathrm{t}(12)=1.14 ; \mathrm{P}=0.28)$ higher for the Abcg5-/- mice in comparison with that of the Abcg5+/+ mice. Furthermore, T1 and T2 exploration times were not significantly different between the genotypes (Fig.2b). Hence, increased plant sterol levels in the brain of the Abcg5-/- mice did not result in detectable effects on object memory performance at the $1 \mathrm{~h}$ inter-trial interval.

a

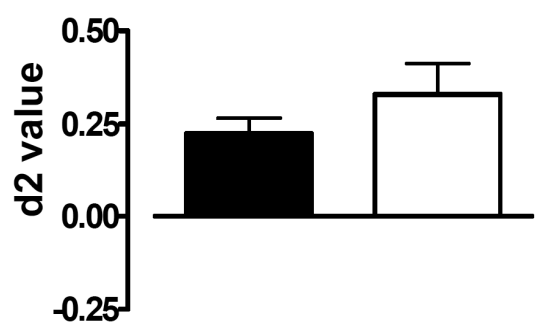

b

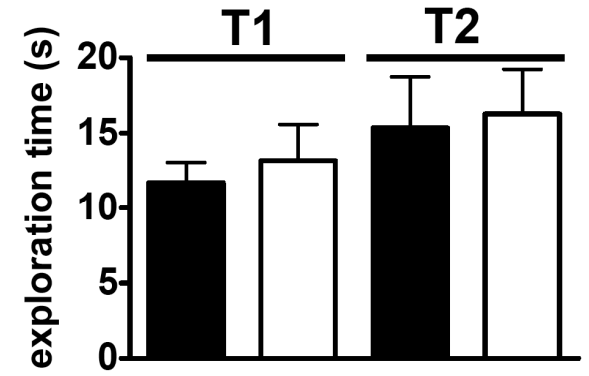

Abcg5

$+/+$

$-1-\quad+/+\quad-/-$

Fig. 2. The performance of Abcg5-/- compared to Abcg5+/+ mice on normal chow diet in the ORT.

The Abcg5 genotype had no influence on object memory performance in an ORT with a $1 \mathrm{~h}$ delay between $\operatorname{Tr} 1$ and $\operatorname{Tr} 2$. Animals that did not reach the minimum of $5 \mathrm{~s}$ exploration in ORT in either $\operatorname{Tr} 1$ or Tr2 were excluded from d2 analysis (exclusion numbers: Abcg5-/- $(\mathrm{n}=1)$ and Abcg5+/+ $(n=1)$ ) (Sik et al, 2003). Extreme values were excluded by means of Dixon's principles of exclusion of extreme values (exclusion numbers: Abcg5-/- $(n=0)$ and Abcg5 $+/+(n=1 ; d 2=0.60)$ ) $[44,45]$. No differences in $d 2$ values could be detected between Abcg5-/- $(\mathrm{n}=7)$ and Abcg5+/+ $(\mathrm{n}=7)$ (a). T1(s) and T2(s) exploration times in respectively $\operatorname{Tr} 1$ and $\operatorname{Tr} 2$ were not different between Abcg5-/- $(\mathrm{n}=8)$ and Abcg5+/+ $(\mathrm{n}=9)(\mathrm{b})$. Values are displayed as mean +/- SEM. 
Subsequently, both groups of mice were subjected to MWEM. As shown in Figure 3a, Abcg5-/- and Abcg5+/+ mice located the platform well $(F(5,90)=31.58$; $\mathrm{P}<0.001)$. There were no significant differences in the acquisition time curves in the trials performed over the different experimental days between the two genotypes (Fig. 3a). In parallel to the acquisition times, the acquisition distance was measured. The distance covered to reach the platform in both groups of mice decreased progressively over the experimental days $(\mathrm{F}(5,90)=22.02 ; \mathrm{P}<0.001)$, whereas there was no difference detected between the genotypes (Fig. 3c). At day $6,1 \mathrm{~h}$ after the last acquisition trial, a $1 \mathrm{~h}$ probe trial was performed. As shown in figure $3 \mathrm{~d}$ and $3 \mathrm{e}$, both Abcg5-/- and Abcg5+/+ mice spent significantly more time in the target quadrant compared to the other quadrants (Abcg5-/-: $\mathrm{F}(3,28)=13.18$; $\mathrm{P}<0.001$ : $\mathrm{PH}$ : target versus left $(\mathrm{t}(14)=3.54 ; \mathrm{P}<0.01)$, right $(\mathrm{t}(14)=5.42 ; \mathrm{P}<0.001)$ and opposite $(\mathrm{t}(14)=5.47 ; \mathrm{P}<0.001)$; and Abcg5+/+: $\mathrm{F}(3,32)=26,82 ; \mathrm{P}<0.001$ : $\mathrm{PH}$ : target versus left $(\mathrm{t}(16)=5.80 ; \mathrm{P}<0.001)$, right $(\mathrm{t}(16)=7.58 ; \mathrm{P}<0.001)$ and opposite $(\mathrm{t}(16)=7.89$; $\mathrm{P}<0.001)$ ), indicating both groups memorized the prior location of the platform.

Since there was no difference between the two groups, it can be concluded that neither object- nor spatial memory was affected by increased brain plant sterol levels. Remarkably, the swimming speed measured in the MWEM was significantly higher in the Abcg5-/- group than in their wild-type littermates $(\mathrm{F}(1,90)=13.49 ; \mathrm{P}<$ 0.001 , Fig. 3b).

Performance of Abcg5-/- and Abcg5+/+ on normal chow in the EZM, the OF and the TST

In order to examine effects of plant sterol accumulation on anxiety- and moodrelated behavior, mice were subjected to the EZM, the OF and the TST. In the EZM task, the latency time before first entering the closed zone of the arena was longer for the Abcg5-/- mice than the Abcg5+/+ mice, although this effect did not reach statistical significance. After the first entrance of the closed zone, time spent in the closed zone, open zone, inner borders and outer borders did not significantly differ between the two genotypes either (Fig. 4a). In addition, the frequency of entrance in the different zones was comparable (Fig. 4b). Therefore, no significant differences in anxiety related behavior were detected in the Abcg5-/- and Abcg5+/+ mice based on the EZM. 
a

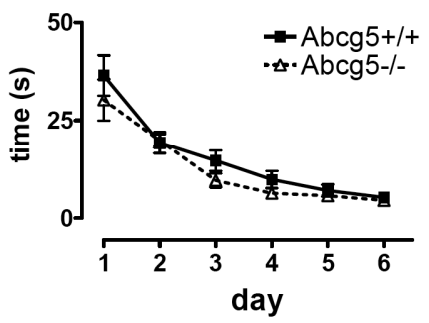

C

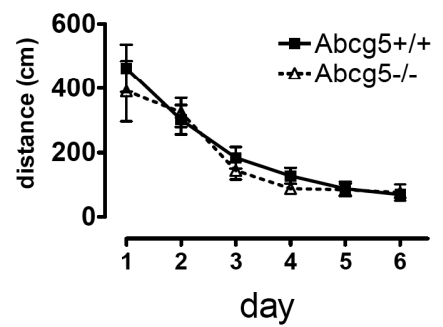

e

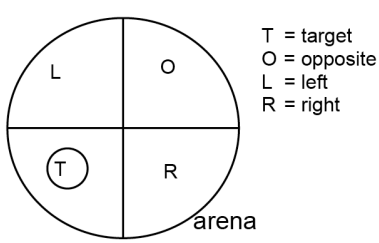

b

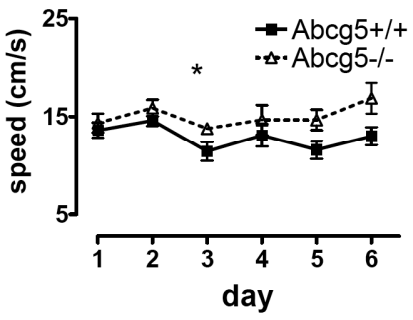

d

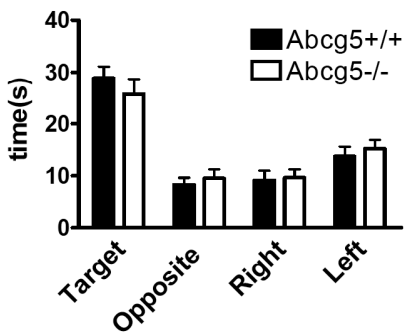

Fig. 3. Spatial learning and memory of Abcg5-/- compared to Abcg5+/+ mice on normal chow diet in the MWEM.

Spatial memory performance did not differ between Abcg5+/+ and Abcg5-/- mice in the MWEM. Acquisition times (a) and distance moved (c) were not different between the Abcg5 $5+/+(n=9)$ and Abcg5-/- $(\mathrm{n}=8)$. Abcg5-/- swim significantly faster than Abcg5+/+ mice (b). In a probe trial without platform, all mice spent significantly more time searching in the target zone and no differences were found between Abcg5+/+ and Abcg5-/- mice (d). Using software the pool was divided into 4 virtual quadrants and a target zone (picture modified from [46]) (e). (Significant differences indicated $*=\mathrm{P}<0.05$ ). Values are displayed as mean $+/$ - SEM. 
a

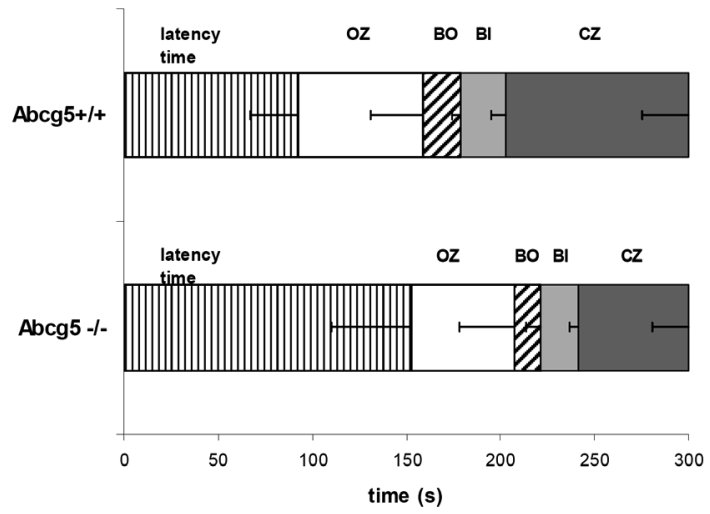

b

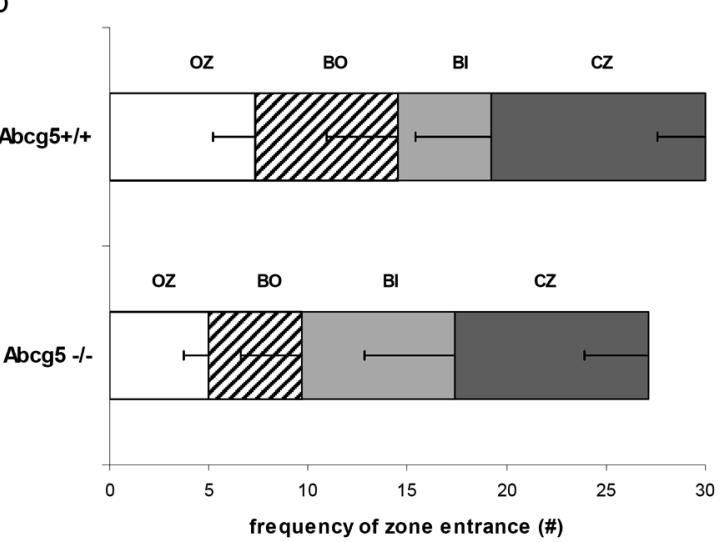

C

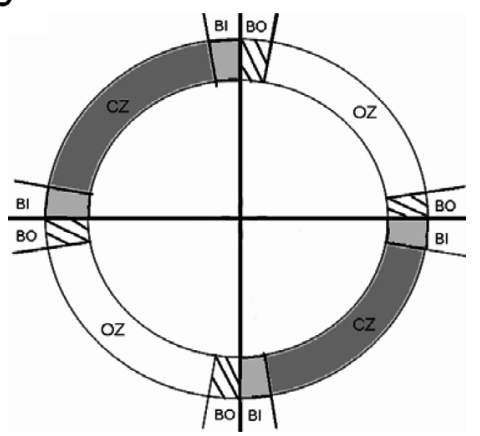

Fig.4. The effect of Abcg5 genotype on anxiety-related behavior in the EZM after being fed a normal chow diet.

The time in zone in the open zone (OZ), the closed parts $(\mathrm{CZ})$, the border-in $(\mathrm{BI})$, the border-out (BO) (indicated in (c)) as well as the latency to enter the CZ (latency time) did not differ between Abcg5+/+ and Abcg5-/- mice in the EZM (a). The frequency of entrance in CZ, OZ, BO and BI were not significantly different either (b). Values are displayed as means - SEM.

In the OF task both Abcg5-/- and Abcg5+/+ mice spent most of their time in the corners and near the walls, whereas the center of the arena was avoided (Abcg5+/+: $\mathrm{F}(2,26)=50.14 ; \mathrm{P}<0.001 ; \mathrm{PH}$ : corners versus center $(\mathrm{t}(16)=6.42 ; \mathrm{P}<0.001)$ and walls versus center $(\mathrm{t}(16)=9.87 ; \mathrm{P}<0.001)$; and Abcg5-/-: $\mathrm{F}(2,20)=14.65 ; \mathrm{P}<0.001$; $\mathrm{PH}$ : corners versus center $(\mathrm{t}(12)=2.82 ; \mathrm{P}<0.05)$ and walls versus center $(\mathrm{t}(12)=5.41$; $\mathrm{P}<0.001)$ ). As shown in Fig. 5a, no significant differences were detected between the Abcg5-/- and Abcg5+/+ mice with respect to the time spent in the center $(\mathrm{t}(14)=1.57 ; \mathrm{P}=0.14)$, the corners $(\mathrm{t}(14)=0.64 ; \mathrm{P}=0.54)$, or near the walls $(\mathrm{t}(14)=1.39 ; \mathrm{P}=0.19)$. Although, there was a difference in swimming speed, the 
locomotor activity measured as the distance moved in a 20 min time interval in the OF task was comparable between the two groups (Abcg5+/+: $8.8 \pm 1.2 \mathrm{~m}$ versus Abcg5-/-: $7.1 \pm 0.5 \mathrm{~m}$; figure $5 \mathrm{~b}$ )
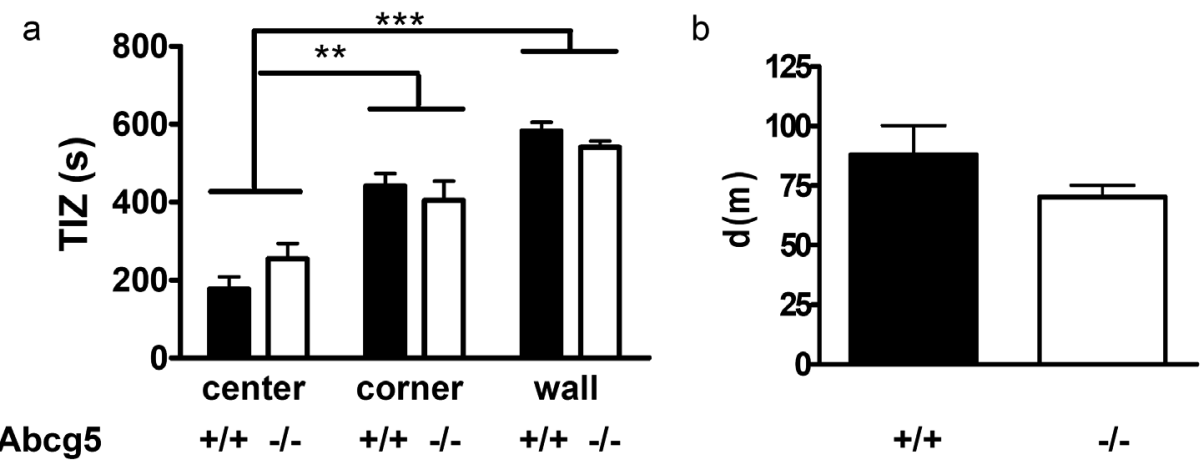

Fig.5. Behavior of the Abcg5-/- and the Abcg5+/+ mice in the OF.

Location pattern was measured for Abcg5+/+ and Abcg5-/- mice spending 20 minutes in the OF. Based on Dixon's principles of exclusion of extreme values [44, 45], 1 animal from the Abcg5-/group was excluded from the analysis $(\mathrm{DM}=29,7 \mathrm{~m})$. Both Abcg5+/+ $(\mathrm{n}=9)$ and Abcg5-/- $(\mathrm{n}=7)$ spent significant less time in the center then in the corners and near the walls (a). No significant difference was measured between the groups in each zone or in total distance moved (DM) (b). (Significant differences indicated $*=\mathrm{P}<0.05$ ). Values are displayed as mean $+/$ - SEM.

Lastly, both groups were subjected to the TST to test antidepressant-like activity of increased presence of plant sterols in the brain. Abcg5-/- $(131.4 \pm 10.8 \mathrm{~s})$ and Abcg5+/+ $(108.9 \pm 20.6 \mathrm{~s})$ mice did not differ with respect to immobility in the TST (data not shown). Overall, the performance in the applied tasks did not reveal any obvious differences in anxiety- or mood- related behavior between Abcg5-/- and Abcg5+/+ mice.

\section{Discussion}

Since it has become clear that plant sterols can accumulate in the brain, it is of major importance to investigate potential consequences of plant sterol accumulation on brain functions. Here we show that accumulation of plant sterols in the brain does have limited consequences on brain cholesterol metabolism in specific brain regions, but does not have any major effects on learning and memory functions, or 
on anxiety and mood related behavior in Abcg5-/- mice in the applied tasks. The Abcg5-/- mice did, however, display increased swimming speed in the MWEM.

As previously reported, in the absence of the Abcg5 gene, plant sterols accumulate not only in serum, but also in the brain [7]. In line with these previous observations, we show that campesterol accumulated preferentially in the brains of Abcg5-/-, but not Abcg5+/+ mice. In addition to the sterol levels described by Plösch et al. [29] and Jansen et al. [7] in serum and total brain hemispheres, we demonstrate that campesterol and sitosterol, relative to the local cholesterol content, accumulated predominantly in the cerebellum. The high plant sterol concentrations in the brain were associated with increased lanosterol levels in the cortex and decreased desmosterol- and 24(S)-OHcholesterol levels in the hippocampus.

Since plant sterols have been reported to activate the LXR-pathway in cultured cells $[19,20,47]$, we further examined their effect on LXR-target genes in the brain. Although Abcg5-/- mice fed a regular chow diet have unaltered LXR-target gene expression, feeding them with a plant sterol-rich diet caused an increased expression of Abcg1 and, to a lesser extent, Abcal mRNA levels in their brains compared with Abcg5+/+ mice. However, other LXR-target genes, including LXR $\alpha$, Apoc1, and Srebp1c were not affected, suggesting the mechanism for their upregulation is not via the LXR-pathway. Apoe was slightly, but not significantly, higher in the Abcg5-/- mice on a high plant sterol diet, while in cultured astrocytes its expression was significantly increased by sitosterol [7]. The difference between the in vivo and in vitro effects may be due to the plant sterol concentrations present. The data indicate that raised plant sterol levels in the brain affect cholesterol metabolism in a brain region specific manner, but do not markedly disturb it.

When fed a high plant sterol diet, $50 \%$ of the Abcg5-/- mice died, while none of the Abcg5+/+ mice did. Recently, it was reported that Abcg5-/- mice on a normal chow diet display macrothrombocytopenia, low platelets counts (32\% of normal) and enlarged spleens (Abcg5-/-: $5.6 \pm 0.5 \mathrm{mg} / \mathrm{g}$ body weight versus Abcg5+/+:3.0 \pm 0.4 $\mathrm{mg} / \mathrm{g}$ body weight) due to their high plant sterol levels [13]. This was also observed in human phytosterolemia patients carrying mutations in either ABCG5 or ABCG8 [48]. In our experiment, Abcg5-/- mice on a high plant sterol diet had even higher spleen weights (Abcg5-/-: $7.5 \pm 0.5 \mathrm{mg} / \mathrm{g}$ body weight versus Abcg5+/+:2.8 \pm 0.3 $\mathrm{mg} / \mathrm{g}$ body weight). Therefore, the increased mortality in the Abcg5-/- mice may be due to increased internal bleeding events due to the macrothrombocytopenia and very low platelet counts trombocytes [13]. However, this requires further examination. 
Our data show that despite the substantially increased plant sterol concentrations and restricted alterations in brain cholesterol metabolism in the Abcg5-/- mice, neither spatial nor object memory were overtly affected. In the present study, the ORT was performed using a $1 \mathrm{~h}$ inter-trial interval in order to reveal a strong overt phenotype. Although, the Abcg5-/- mice performed slightly better in the task, the difference was not significant. Prior power analyses, based on a minimum difference in the $\mathrm{d} 2$ value of 0.18 points to be detected and a standard deviation of 0.09 points, indicated that 6 animals per group would be sufficient. However, since the actual difference between the two groups was less than the predetermined threshold, no significant differences were detected. However, our data-set cannot exclude that an increased sample size would reveal a significant difference at an acceptable power [31]. In retrospect, therefore, calculated sample size could be considered a weakness of this study. It is possible that testing at different inter-trial intervals in the ORT could provide a more complete overview of the performance of Abcg5+/+ and Abcg5-/- animals. In parallel with the ORT, a second probe trial in the MWEM for example, performed at a 24h inter-trial interval, may reveal longterm spatial memory differences between genotypes. Yet, in line with our findings, it was recently shown that 85 weeks of dietary plant sterol supplementation to 43-69 year old statin-treated hypercholesterolemic individuals did not affect cognitive function [49]. In addition, no relation was detected between serum sitosterol and campesterol concentrations and cognitive performance in a healthy aging population, neither at baseline nor after 6 years of follow-up [50]. A correlation with serum lanosterol and lathosterol levels [50] was, however, reported in the same population. Neither serum nor brain latho- and lanosterol levels showed a relationship with object or spatial memory in the Abcg5-/- mice. Serum plant sterol levels in healthy aged subjects with plant sterol supplementation are not as high as the serum plant sterol levels in the Abcg5-/- mice, which are in the same range as the serum plant sterol levels measured in patients with phytosterolemia [51]. Due to the small number of phytosterolemia patients, it is not possible to test the effect of plant sterols on their cognitive functions. Patients with familial hypercholesterolemia on statins in combination with plant sterol supplementation also display strongly increased serum plant sterol levels [52]. Brain plant sterol levels in relation to cognitive functions have hardly been examined in humans. The use of the Abcg5-/- mouse model, however, indicates some differences with phytosterolemic patients; Abcg5-/- mice do not develop premature atherosclerosis and do not show increased serum cholestanol levels [51, 53]. 
Activation of the LXR-pathway by T0901317 for example was found to enhance memory functions in an AD-model, but not in C57BL/6NCrl wild-type mice [21, 54]. Therefore, although there were no significant differences in cognitive endpoints between Abcg5-/- and Abcg5+/+ mice, it remains to be established whether increased brain plant sterols levels have beneficial effects on cognitive function in animal models of neurodegenerative diseases.

As described in the introduction, decreased serum cholesterol concentrations are strongly linked with severe depressive behavior. However, the markedly suppressed serum cholesterol concentrations caused by elevated serum plant sterol concentrations in the Abcg5-/- mice did not affect anxiety and mood related behavior as indicated by their performance in the applied tasks. Plant sterols from the plant species, Tilia americana var. mexicana, have been reported to possess anxiolitic properties, with sitosterol as the identified bioactive anxiolytic compound, having a dose dependent sedative response resembling diazepam [55]. Nonetheless, the strongly increased serum and brain sitosterol concentrations in the Abcg5-/mice were not associated with aberrant behavior in anxiety or mood related tasks. Therefore, our results show no overt effects of strongly elevated brain plant sterol levels on unforced mood or anxiety related behavior in mice. Further studies (e.g. conditioned anxiety tests) are required to elucidate whether other cognitive and emotive domains are similarly unaffected.

Administration of sitosterol to an LXR $\beta-/-$ mouse model caused CNS-dependent locomotor disease resembling amyotrophic lateral sclerosis and Parkinson's disease [56]. Death of motor neurons in the lumbar region of the spinal cord and loss of tyrosine hydroxylase-positive dopaminergic neurons in the substantia nigra followed sitosterol administration in the LXR $\beta^{-/}$mice, but not in wild type controls. In line with the results obtained from sitosterol fed wild-type mice, our Abcg5-/mice did not show aberrations in locomotor behavior in the OF. On the contrary, Abcg5-/- mice displayed a significantly raised swimming speed during the acquisition trials in the MWEM compared with their Abcg5+/+ littermates. Since plant sterols preferentially accumulate in the cerebellum of Abcg5-/- mice, a rotarod coordination function assay might lead to better insights into the mechanism of this effect [57].

Alterations in brain cholesterol homeostasis can be associated with neurological problems. Therefore, it is of major importance that we demonstrate that strongly elevated serum and brain plant sterol concentrations in a phytosterolemia mouse model, Abcg5-/- mice do not result in an overt negative phenotype in behavioral, 
e.g. learning and memory, and anxiety and mood in the applied behavioral tasks. An exception was increased swimming speed in the MWEM in the Abcg5-/- mice in comparison with the Abcg5+/+ mice. Further research on the consequences of plant sterol administration in human neurodegenerative disease with cognitive decline is required.

\section{Acknowledgements}

We are grateful to Professor Dr. Gerald Salen and Dr. Patric Delhanty for critically reading the manuscript. We would like to thank Juul Baller for his excellent technical assistance. The project was financially supported by Marie Curie Early Stage Training Fellowship: Contract number MEST-CT-2005-02058. All experimental procedures concerning the used mice were approved by the local ethical committee of the Maastricht University for animal experiments according to governmental guidelines. There are no actual conflicts of interest. 


\section{References}

1. Thompson, G.R. and S.M. Grundy, History and development of plant sterol and stanol esters for cholesterol-lowering purposes. Am J Cardiol, 2005. 96(1A): p. 3D-9D.

2. Calpe-Berdiel, L., J.C. Escola-Gil, and F. Blanco-Vaca, New insights into the molecular actions of plant sterols and stanols in cholesterol metabolism. Atherosclerosis, 2008.

3. Clifton, P., Lowering cholesterol - a review on the role of plant sterols. Aust Fam Physician, 2009. 38(4): p. 218-21.

4. Pollak, O.J. and D. Kritchevsky, Sitosterol. Monogr Atheroscler, 1981. 10: p. 1-219.

5. Salen, G., E.H. Ahrens, Jr., and S.M. Grundy, Metabolism of beta-sitosterol in man. J Clin Invest, 1970. 49(5): p. 952-67.

6. $\quad$ Fricke, C.B., et al., Increased plant sterol and stanol levels in brain of Watanabe rabbits fed rapeseed oil derived plant sterol or stanol esters. Br J Nutr, 2007. 98(5): p. 890-9.

7. Jansen, P.J., et al., Dietary plant sterols accumulate in the brain. Biochim Biophys Acta, 2006.

8. $\quad \mathrm{Yu}, \mathrm{L}$., et al., Disruption of Abcg5 and Abcg8 in mice reveals their crucial role in biliary cholesterol secretion. Proc Natl Acad Sci U S A, 2002. 99(25): p. 16237-42.

9. $\quad$ Berge, K.E., et al., Accumulation of dietary cholesterol in sitosterolemia caused by mutations in adjacent ABC transporters. Science, 2000. 290(5497): p. 1771-5.

10. Lee, M.H., et al., Identification of a gene, ABCG5, important in the regulation of dietary cholesterol absorption. Nat Genet, 2001. 27(1): p. 79-83.

11. Plosch, T., et al., Increased hepatobiliary and fecal cholesterol excretion upon activation of the liver X receptor is independent of ABCA1. J Biol Chem, 2002. 277(37): p. 33870-7.

12. Bhattacharyya, A.K. and W.E. Connor, Beta-sitosterolemia and xanthomatosis. A newly described lipid storage disease in two sisters. J Clin Invest, 1974. 53(4): p. 1033-43.

13. Kruit, J.K., et al., Plant sterols cause macrothrombocytopenia in a mouse model of sitosterolemia. J Biol Chem, 2008. 283(10): p. 6281-7.

14. Miettinen, T.A., Phytosterolaemia, xanthomatosis and premature atherosclerotic arterial disease: a case with high plant sterol absorption, impaired sterol elimination and low cholesterol synthesis. Eur J Clin Invest, 1980. 10(1): p. 27-35.

15. Salen, G., et al., Sitosterolemia. J Lipid Res, 1992. 33(7): p. 945-55.

16. Shulman, R.S., et al., Beta-sitosterolemia and xanthomatosis. N Engl J Med, 1976. 294(9): p. 4823.

17. Maxfield, F.R. and I. Tabas, Role of cholesterol and lipid organization in disease. Nature, 2005. 438(7068): p. 612-21.

18. Tint, G.S., et al., Markedly increased tissue concentrations of 7-dehydrocholesterol combined with low levels of cholesterol are characteristic of the Smith-Lemli-Opitz syndrome. J Lipid Res, 1995. 36(1): p. 89-95.

19. Plat, J., J.A. Nichols, and R.P. Mensink, Plant sterols and stanols: effects on mixed micellar composition and LXR (target gene) activation. J Lipid Res, 2005. 46(11): p. 2468-76.

20. Yang, C., et al., Disruption of cholesterol homeostasis by plant sterols. J Clin Invest, 2004. 114(6): p. 813-22.

21. Vanmierlo, T., et al., Liver $X$ receptor activation restores memory in aged AD mice without reducing amyloid. Neurobiol Aging, 2009.

22. Dufour, F., et al., Cholesterol-enriched diet affects spatial learning and synaptic function in hippocampal synapses. Brain Res, 2006. 1103(1): p. 88-98.

23. Mulder, M., et al., Low-density lipoprotein receptor-knockout mice display impaired spatial memory associated with a decreased synaptic density in the hippocampus. Neurobiol Dis, 2004. 16(1): p. 212-9.

24. Papakostas, G.I., et al., Cholesterol in mood and anxiety disorders: review of the literature and new hypotheses. Eur Neuropsychopharmacol, 2004. 14(2): p. 135-42.

25. Kaplan, J.R., et al., Demonstration of an association among dietary cholesterol, central serotonergic activity, and social behavior in monkeys. Psychosom Med, 1994. 56(6): p. 479-84.

26. Engelberg, H., Low serum cholesterol and suicide. Lancet, 1992. 339(8795): p. 727-9.

27. Heron, D.S., et al., Lipid fluidity markedly modulates the binding of serotonin to mouse brain membranes. Proc Natl Acad Sci U S A, 1980. 77(12): p. 7463-7.

28. Scanlon, S.M., D.C. Williams, and P. Schloss, Membrane cholesterol modulates serotonin transporter activity. Biochemistry, 2001. 40(35): p. 10507-13. 
29. Plosch, T., et al., Sitosterolemia in ABC-transporter G5-deficient mice is aggravated on activation of the liver-X receptor. Gastroenterology, 2004. 126(1): p. 290-300.

30. $\quad$ Rutten, K., et al., The selective PDE5 inhibitor, sildenafil, improves object memory in Swiss mice and increases cGMP levels in hippocampal slices. Behav Brain Res, 2005. 164(1): p. 11-6.

31. Sik, A., et al., Performance of different mouse strains in an object recognition task. Behav Brain Res, 2003. 147(1-2): p. 49-54.

32. Blokland, A., E. Geraerts, and M. Been, A detailed analysis of rats' spatial memory in a probe trial of a Morris task. Behav Brain Res, 2004. 154(1): p. 71-5.

33. Mulder, M., et al., Apolipoprotein E protects against neuropathology induced by a high-fat diet and maintains the integrity of the blood-brain barrier during aging. Lab Invest, 2001. 81(7): p. 953-60.

34. Shepherd, J.K., et al., Behavioural and pharmacological characterisation of the elevated "zeromaze" as an animal model of anxiety. Psychopharmacology (Berl), 1994. 116(1): p. 56-64.

35. Kulkarni, S.K., K. Singh, and M. Bishnoi, Elevated zero maze: a paradigm to evaluate antianxiety effects of drugs. Methods Find Exp Clin Pharmacol, 2007. 29(5): p. 343-8.

36. van Donkelaar, E.L., et al., Acute tryptophan depletion in C57BL/6 mice does not induce central serotonin reduction or affective behavioural changes. Neurochem Int, 2009.

37. Steckler, T., et al., Effects of $m$ Glul receptor blockade on anxiety-related behaviour in the rat lick suppression test. Psychopharmacology (Berl), 2005. 179(1): p. 198-206.

38. Parfitt, D.B., et al., Early life stress effects on adult stress-induced corticosterone secretion and anxiety-like behavior in the C57BL/6 mouse are not as robust as initially thought. Horm Behav, 2007. 52(4): p. 417-26.

39. Cryan, J.F., C. Mombereau, and A. Vassout, The tail suspension test as a model for assessing antidepressant activity: review of pharmacological and genetic studies in mice. Neurosci Biobehav Rev, 2005. 29(4-5): p. 571-625.

40. Steru, L., et al., The automated Tail Suspension Test: a computerized device which differentiates psychotropic drugs. Prog Neuropsychopharmacol Biol Psychiatry, 1987. 11(6): p. 659-71.

41. Lutjohann, D., et al., Profile of cholesterol-related sterols in aged amyloid precursor protein transgenic mouse brain. J Lipid Res, 2002. 43(7): p. 1078-85.

42. Heid, C.A., et al., Real time quantitative PCR. Genome Res, 1996. 6(10): p. 986-94.

43. Vanmierlo, T., et al., Alterations in Brain Cholesterol Metabolism in the APPSLxPS1mut mouse, a Model for Alzheimer's Disease. J Alzheimers Dis, 2009.

44. Dixon, W.J., Analyses of extreme values. Annals of Mathematical Statistics, 1959. 21: p. 488-506.

45. Dixon, W.J., Ratios involving extreme values. Annals of Mathematical Statistics, 1959. 22: p. 6878.

46. Blokland, A., K. Rutten, and J. Prickaerts, Analysis of spatial orientation strategies of male and female Wistar rats in a Morris water escape task. Behav Brain Res, 2006. 171(2): p. 216-24.

47. Kaneko, E., et al., Induction of intestinal ATP-binding cassette transporters by a phytosterolderived liver X receptor agonist. J Biol Chem, 2003. 278(38): p. 36091-8.

48. Rees, D.C., et al., Stomatocytic haemolysis and macrothrombocytopenia (Mediterranean stomatocytosis/macrothrombocytopenia) is the haematological presentation of phytosterolaemia. Br J Haematol, 2005. 130(2): p. 297-309.

49. Schiepers, O.J., et al., Consuming Functional Foods Enriched with Plant Sterol or Stanol Esters for 85 Weeks Does Not Affect Neurocognitive Functioning or Mood in Statin-Treated Hypercholesterolemic Individuals. J Nutr, 2009.

50. Teunissen, C.E., et al., Serum cholesterol, precursors and metabolites and cognitive performance in an aging population. Neurobiol Aging, 2003. 24(1): p. 147-55.

51. Salen, G., et al., Increased plasma cholestanol and 5 alpha-saturated plant sterol derivatives in subjects with sitosterolemia and xanthomatosis. J Lipid Res, 1985. 26(2): p. 203-9.

52. Ketomaki, A., H. Gylling, and T.A. Miettinen, Non-cholesterol sterols in serum, lipoproteins, and red cells in statin-treated FH subjects off and on plant stanol and sterol ester spreads. Clin Chim Acta, 2005. 353(1-2): p. 75-86.

53. Salen, G., et al., Lethal atherosclerosis associated with abnormal plasma and tissue sterol composition in sitosterolemia with xanthomatosis. J Lipid Res, 1985. 26(9): p. 1126-33.

54. Riddell, D.R., et al., The LXR agonist TO901317 selectively lowers hippocampal Abeta42 and improves memory in the Tg2576 mouse model of Alzheimer's disease. Mol Cell Neurosci, 2007. 34(4): p. 621-8.

55. Aguirre-Hernandez, E., et al., Bioactivity-guided isolation of beta-sitosterol and some fatty acids as active compounds in the anxiolytic and sedative effects of Tilia americana var. mexicana. Planta Med, 2007. 73(11): p. 1148-55. 


\section{Chapter V}

56. Kim, H.J., et al., Liver X receptor beta (LXRbeta): a link between beta-sitosterol and amyotrophic lateral sclerosis-Parkinson's dementia. Proc Natl Acad Sci U S A, 2008. 105(6): p. 2094-9.

57. Crawley, J.N., Behavioral phenotyping of transgenic and knockout mice: experimental design and evaluation of general health, sensory functions, motor abilities, and specific behavioral tests. Brain Res, 1999. 835(1): p. 18-26. 



\section{Chapter VI}

\section{The Plant Sterol Brassicasterol as Biomarker in Alzheimer's Disease}




\section{Chapter VI}

\section{The Plant Sterol Brassicasterol as Biomarker in Alzheimer's Disease}

Tim Vanmierlo a,b,f , Julius Popp ${ }^{\mathrm{c}}$, Heike Kölsch ${ }^{\mathrm{c}}$, Silvia Friedrichs ${ }^{\mathrm{a}}$, Frank Jessen c,f Thomas Bertsch ${ }^{\mathrm{d}}$, Tobias Hartmann ${ }^{\mathrm{e}}$, Wolfgang Maier ${ }^{\mathrm{c}}$, Harry Steinbusch ${ }^{\mathrm{b}, \mathrm{f}}$, Monique Mulder ${ }^{\mathrm{d}}$ and Dieter Lütjohann ${ }^{\mathrm{a}, \mathrm{f}^{\prime}}$

${ }^{a}$ Institute of Clinical Chemistry and Pharmacology, University Clinics Bonn, Germany; ${ }^{b}$ Department of Neuroscience, Maastricht University, The Netherlands; ${ }^{\mathrm{c}}$ Department of Psychiatry, University of Bonn, Germany; ${ }^{\mathrm{d}}$ Department of Internal Medicine, Division of Pharmacology, Vascular and Metabolic Diseases, Erasmus

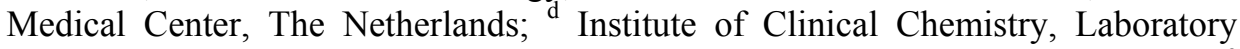
Medicine and Transfusion Medicine, Nuremberg Clinic, Nuremberg Germany; German Institute for Dementia Prevention, Neurodegeneration and Neurobiology, Saarland University, Homburg/Saar, Germany; ${ }^{f}$ EURON (European Graduate School for Neuroscience)

Submitted 


\section{Abstract}

Plant sterols are solely dietary derivable sterols which are structurally very likely to cholesterol. In contrast to peripheral cholesterol, plant sterols can cross the bloodbrain barrier and can accumulate within mammalian brains. Since disturbances in cerebrospinal fluid (CSF)-blood barrier are linked to $\mathrm{AD}$, we investigated if this results in altered plant sterol concentrations in CSF and if these are potential biomarkers for AD. Applying gas chromatography/mass spectrometry analysis plant sterol concentrations were measured in plasma and CSF of AD patients $(n=67)$ and non-demented controls $(\mathrm{n}=29)$. Age, gender, serum vs. CSF albumin ratio, CSF $\mathrm{A} \beta_{42}$, CSF-phospho-tau, APOE4 genotype and serum creatinine were introduced in a backwards linear regression model to determine predictive covariates for the individual plant sterols. The proven relevant predictors were introduced as covariates in a univariate analysis of variance in order to compare plasma and CFS plant sterol concentrations between the two experimental populations. Albumin quotient appeared a consistent and strong predictor in CSF for both cholesterol and the methyl-plant sterols campesterol and brassicasterol. Phospho-tau was a consistent predictor for ethyl-plant sterols sitosterol and stigmasterol in CSF of AD patients. The presence of an APOE4 allele was the only consistent predictor of all plant sterols and cholesterol in plasma. Comparison of the lipid parameters per diagnose based on the relevant predictors in the univariate analysis of variance revealed significantly lower concentrations of brassicasterol $(\mathrm{P}<0.001)$ and sitosterol $(\mathrm{P}<0.05)$ in $\mathrm{CSF}$ of $\mathrm{AD}$ patients in comparison with controls. After correction for CSF cholesterol content, brassicasterol $(\mathrm{P}<0.01)$ remained significantly lower in CSF of AD patients, representing a relevant biomarker for AD.

\section{Introduction}

At present, Alzheimer's disease (AD) patients can not be distinguished from controls based on one single biomarker. The most sturdy biomarkers for late onset $\mathrm{AD}(\mathrm{LOAD})$ are brain atrophy, lowered concentrations of amyloid $\beta\left(\mathrm{A} \beta_{42}\right)$, increased concentrations of total microtubule-associated tau protein ( $\mathrm{t}$-tau), and increased hyperphosphorylated tau at threonine 181 (phospho-tau) in CSF [1-6]. However, none of these biomarkers is fully unassailable or conclusive. Therefore, 
we believe that additional biomarkers are required to constitute a gamma of easily accessible parameters which together posses an increasingly predictive power in making a more correct and early AD diagnosis.

The brain cholesterol metabolism is a balanced and well regulated complex homeostatic system [7]. The brain has virtually no access to circulating cholesterol concentrations and is consequently almost entirely dependent on in situ cholesterol synthesis [8]. The choroid plexus filters and actively secretes compounds from the plasma into the CSF and thereby predominantly determines the CSF composition [9]. Plant sterols comprise a group of exogenous sterols that enter the body only via the diet. Relatively large amounts of plant sterols are present in plant oils, nuts, and nowadays plant sterol enriched dairy products. The chemical structure of plant sterols is very similar to that of cholesterol. They differ from cholesterol only by an additional methyl-group (campesterol) or ethyl-group (sitosterol) at the C24position, or by an additional double bond at the C22-position (brassicasterol or stigmasterol, respectively) $[10,11]$. Yet, this small structural difference leads to very divergent metabolic fates of plant sterols and cholesterol in mammals. In contrast to plasma cholesterol, plant sterols cross the blood-brain barrier and accumulate within the brain $[12,13]$. Plasma accumulation of plant sterols upon temporally increased feeding of a plant sterol enriched diet in mice resulted in an irreversible accumulation in the brain, more in particular within the membrane lipid rafts of the neurons (chapter IV). Female gender, high mid-life plasma cholesterol, decreased CSF A $\beta 42$, increased CSF phospho-tau and an impaired blood-CSF barrier permeability at the level of the choroid plexus, all have been described to be risk factors of $\mathrm{AD}[1,14-18]$. Based on the solely exogenous nature of plant sterols in the CSF and the impaired choroid plexus function in $\mathrm{AD}$, we hypothesize that plant sterol concentrations in CSF are altered in AD pathology and therefore can be added to the relevant spectrum biomarkers for AD. CSF and plasma plant sterol concentration were compared between a group of diagnosed $\mathrm{AD}$ patients and an age matched control group with age, gender, apoE, albumin quotient, A $\beta 42$ and phospho-tau as covariates. 


\section{Materials and Methods}

\section{Study population}

After overnight fasting, CSF and plasma samples of $67 \mathrm{AD}$ patients and 29 nondemented controls were obtained by respectively lumbar puncture and venous puncture. AD patients were recruited from the Department of Psychiatry, University of Bonn, Germany. Non-demented controls were patients, referred to the Department of Neurology, University of Bonn, who underwent lumbar puncture during clinical routine diagnosis of neurological disorders. Patient characteristics are given in Table I. AD patients were diagnosed according to DSM-IV, supported by clinical examination, detailed structured interviews, neuropsychological testing, cognitive screening and neuroimaging studies. The cognitive status of the control group was confirmed by MMSE (Table I) [19]. ApoE4, Albumin Ratio (Ralb) [CSF albumin (milligrams/ liter) divided by plasma albumin (grams/liter)*1000], CSF A 342 , CSF phospho-tau and plasma creatinine were measured within the population. Serum creatinine was measured during the clinical routine. Quantitative assessment of serum and CSF albumin was performed during the clinical routine. CSF Aß1-42 and P-tau181 concentrations were measured by ELISA using commercially available assays (Innogenetics, Gent, Belgium). Leukocyte genomic DNA was isolated from $10 \mathrm{ml}$ EDTA blood with the Qiagen blood isolation kit (Qiagen, Hilden, Germany) and the APOE genotype was determined.

Since so far plasma creatinine was not associated with CSF sterol concentrations and $\mathrm{AD}$ pathology before, creatinine was included as negative control in the backwards linear regression analysis as initial covariate. Subjects with symptomatic cardiac disease, renal or hepatic dysfunction, insulin-dependent diabetes mellitus, untreated thyroidal dysfunction, inflammatory disease, blood-brain barrier (BBB) disturbance (Ralb > 9), depression and other neurodegenerative disorders or alcohol abuse were excluded from this study. Also patients receiving treatment with known cholesterol lowering medication were excluded from this study.

All participants of the study gave informed written consent. The study has been approved by the Ethics Committee of the Faculty of Medicine of the University Clinics of Bonn, Germany. 
Table 1: Characteristics of AD patients and controls

\begin{tabular}{llll}
\hline Patients & Variables & Values & P \\
\hline Healthy & MMSE (score on 30) & $29.4 \pm 0.7$ & T-test: $\mathrm{P}<0.001$ \\
AD patients & & $21.3 \pm 4.6$ & \\
\hline Healthy & $\beta 1:$ Age (years) & $69.0 \pm 6.9$ & T-test: $\mathrm{P}=0.08$ \\
AD patients & & $71.8 \pm 7.5$ & \\
\hline Healthy & $\beta 2:$ Gender $(\%$ female) & $62.7 \%$ & $\chi^{2}$-test: $\mathrm{P}=0.11$ \\
AD patients & & $44.8 \%$ & \\
\hline Healthy & $\beta 3:$ APOE4 allele carrier & $13.8 \%$ & $\chi^{2}$-test: $\mathrm{P}<0.001$ \\
AD patients & & $58.2 \%$ & \\
\hline Healthy & $\beta 4:$ CSF:plasma & $6.41 \pm 2.35$ & T-test: $\mathrm{P}<0.05$ \\
AD patients & Albumin quotient x $10^{3}$ & $5.17 \pm 2.14$ & \\
\hline Healthy & $\beta 5:$ A $\beta 42$ CSF $(\mathrm{pg} / \mathrm{ml})$ & $884.2 \pm 164.4$ & T-test: $\mathrm{P}<0.001$ \\
AD patients & & $458.6 \pm 241.1$ & \\
\hline Healthy & $\beta 6:$ P-tau CSF $(\mathrm{pg} / \mathrm{ml})$ & $49.0 \pm 13.5$ & T-test: $\mathrm{P}<0.001$ \\
AD patients & & $94.5 \pm 44.9$ & \\
\hline Healthy & $\beta 7:$ plasma creatinine & $1.07 \pm 1.17$ & T-test: $\mathrm{P}<0.05$ \\
AD patients & mg/dl & $0.83 \pm 0.18$ & \\
\hline
\end{tabular}

Diagnosis related mini mental state examination (MMSE) and covariates ( $\beta$ ) for AD patients $(n=67)$ and non-demented (healthy) controls $(n=29)$ were displayed as mean $+/-$ SD.

\section{Sterol extraction and analysis from plasma and CSF samples}

CSF was collected by diagnostic lumbar punctures at the Departments of Neurology or Psychiatry, University of Bonn. A standardized technique with a $22 \mathrm{G}$ "atraumatical" spinal needle and a sitting or lying position for the patient was applied. The CSF samples were kept on ice for a maximum of $1 \mathrm{~h}$ until being centrifuged for $10 \mathrm{~min}$ at $2000 \mathrm{~g}$ at $4^{\circ} \mathrm{C}$. Samples were aliquoted to $0.25 \mathrm{ml}$ and were stored in polypropylene tubes at $-80{ }^{\circ} \mathrm{C}$ until assay procedures. CSF and plasma concentrations of campesterol, brassicasterol, sitosterol and stigmasterol and CSF cholesterol were measured by a modified sensitive method using combined gas 
chromatography/mass spectrometry (GC/MS) as described previously [20]. Plasma concentrations of cholesterol were measured by gas chromatography/flame ionization detection (GC/FID). $50 \mu \mathrm{g} 5 \alpha$-cholestane (Serva) $(50 \mu \mathrm{L}$ from a stock solution of $5 \alpha$-cholestane in cyclohexane; $1 \mathrm{mg} / \mathrm{mL}$ ) and $1 \mu \mathrm{g}$ epicoprostanol (Sigma) $(10 \mu \mathrm{L}$ froma stock solution epicoprostanol in cyclohexane; $100 \mu \mathrm{g} / \mathrm{mL})$ were added to $100 \mu \mathrm{L}$ plasma and $0.5 \mu \mathrm{g}$ epicoprostanol ( $5 \mu \mathrm{L}$ from a stock solution epicoprostanol in cyclohexane; $100 \mu \mathrm{g} / \mathrm{mL}$ ) was added to $250 \mu \mathrm{L}$ CSF. $1 \mathrm{~mL} \mathrm{NaOH}$ (1M) in $80 \%$ ethanol, alkaline hydrolysis was performed for $60 \mathrm{~min}$ at $61{ }^{\circ} \mathrm{C}$, and the sterols were subsequently extracted with $3 \mathrm{~mL}$ of cylcohexane twice. The organic solvents were evaporated and the residual plasma sterols were dissolved in $160 \mu \mathrm{L}$ n-decane. $80 \mu \mathrm{L}$ of the plasma $\mathrm{n}$-decane samples were transferred into micro-vials for GC/MS quantification of plant sterols. The sterols were derivatized to trimethylsilyl (TMSi) ethers by adding $10 \mu \mathrm{L}$ TMSi-reagent (pyridine: hexamethylsisilazane- rimethylchlorosilane; $9: 3: 1$, by volume; all reagents were applied from Merck) and incubated for $1 \mathrm{~h}$ at $64{ }^{\circ} \mathrm{C}$.

The residual $80 \mu \mathrm{L}$ of the plasma n-decane samples were diluted with $300 \mu \mathrm{L} \mathrm{n}$ decane and derivatized with $30 \mu \mathrm{L}$ TMSi-reagent preceding analysis of cholesterol by gas chromatography-flame ionization detection (GC/FID). The sterols from CSF were dissolved in $50 \mu \mathrm{L}$-decane and transferred into micro-vials for GC/MS of cholesterol and plant sterols.

\section{GC/FID and $G C / M S$}

Plasma cholesterol was quantified by GC/FID on an HP 6890 series II plus GC (Agilent Technologies, Böblingen, Germany) using $5 \alpha$-cholestane as an internal standard. An aliquot of $2 \mu \mathrm{L}$ was injected in a splitless mode at $280{ }^{\circ} \mathrm{C}$ by an automated sampler and injector (HP 7683). Hydrogen was used as carrier gas with an inlet pressure of $9.9 \mathrm{psi}$, resulting in a total gas-flow of $1.1 \mathrm{~mL} / \mathrm{min}$ and the temperature of the flame ionization detector was kept at $280{ }^{\circ} \mathrm{C}$. The sterols were separated on a cross-linked methyl silicone DB-XLB 122-1232 fused silica capillary column (J\&W, Folsom, USA) $(30 \mathrm{~m} \times 0.25 \mu \mathrm{mm}$ i.e. $\times 0.25 \mu \mathrm{m}$ film thickness) in an Hewlett-Packard (HP 6890) gas chromatograph. The oven temperature was initially kept at $150{ }^{\circ} \mathrm{C}$ for $3 \mathrm{~min}$, and then gradually increased to a final temperature of $290{ }^{\circ} \mathrm{C}$. The ratios of the cholesterol areas to the area of internal standard were calculated and multiplied by the added amount of the internal standard ( $50 \mu \mathrm{g} 5 \alpha$ - cholestane) to reveal absolute cholesterol concentrations.

GC/MS was performed on an HP GC-MSD system (HP 5890 series II GC) combined with a 5971 mass selective detector (Agilent Technologies, Böblingen, Germany) equipped with a DB-XLB 122-1232 fused silica capillary column (J\&W, 
Folsom, USA) $(30 \mathrm{~m} \times 0.25 \mathrm{~mm}$ i.e. $\times 0.25 \mu \mathrm{m}$ film thickness $)$ in the splitless mode using helium $(1 \mathrm{~mL} / \mathrm{min})$ as the carrier gas. The temperature program was as follows: $150{ }^{\circ} \mathrm{C}$ for $1 \mathrm{~min}$, followed by $20^{\circ} \mathrm{C} / \mathrm{min}$ up to $260^{\circ} \mathrm{C}$, and $10^{\circ} \mathrm{C} / \mathrm{min}$ upto $280{ }^{\circ} \mathrm{C}$ (for $15 \mathrm{~min}$ ). Neutral sterols were monitored as their TMSi derivatives in the selected ionmonitoringmode using the followingmasses: epicoprostanol (internal standard) $\mathrm{m} / \mathrm{z} 370$ [M-OTMSi] $]^{+}$, cholesterol at $\mathrm{m} / \mathrm{z} 458[\mathrm{M}]^{+}$, campesterol $\operatorname{atm} / \mathrm{z} 472[\mathrm{M}]^{+}$, brassicasterol at $\mathrm{m} / \mathrm{z} 470[\mathrm{M}]^{+}$, sitosterol at $\mathrm{m} / \mathrm{z} 486[\mathrm{M}]^{+}$and stigmasterol at $\mathrm{m} / \mathrm{z} 484[\mathrm{M}]^{+}$. Identity of all sterols was proven by comparison with the full-scan mass spectra of authentic compounds (range, m/z 50-500). All the above determined plant sterols were sufficiently separated on the column from additional cholesterol precursors and metabolites present in serum or in CSF samples. Accuracy of the method was established by recovery experiments, day to day variation (below 3\%), limit of detection and limit of quantification below the present concentrations for each sterol.

\section{Statistical analysis}

Since plant sterols in plasma correlate with intestinal cholesterol absorption rates, plant sterols in plasma and CSF are additionally corrected for cholesterol concentrations. These data are given as ratios of plant sterol/cholesterol in Table IV. The correlation of plant sterols and cholesterol was investigated by Pearson's correlation analysis. The predictive value of potential covariates age $(\beta 1)$, gender $(\beta 2)$, the APOE4 allele $(\beta 3)$, albumin quotient $(\beta 4), A \beta 42(\beta 5)$, phospho-tau $(\beta 6)$, and creatinine concentration $(\beta 7)$ for the individual plant sterol concentrations in CSF and plasma were determined by backwards linear regression analysis for total population and split population by diagnosis. Consequently, the corresponding predictive covariates were included in a univariate analysis by diagnose to evaluate absolute levels of cholesterol and plant sterols and corresponding ratios of plant sterol / cholesterol for both CSF and plasma parameters (SPSS, V 15.0). P-values were set two sided: $*=\mathrm{P}<0.05, * *=\mathrm{P}<0.01, * * *=\mathrm{P}<0.001$.

\section{Results}

$\mathrm{AD}$ and healthy control patients were age and gender matched. The presence of the APOE4 allele and CSF phospho-tau concentrations were significantly higher in the AD group compared to the healthy controls, whereas CSF A $\beta 42$ was significantly lower. Albumin quotient and plasma creatinine concentrations were slightly, but significantly higher in the healthy control group (Table 1). 
Backwards linear regression analysis with age, gender, presence of APOE4, albumin quotient, $\mathrm{A} \beta 42$, phospho-tau and creatinine as initial covariates was performed to reveal which covariates are predictive for the individual absolute plant sterol concentrations in CSF and plasma (Table 2). In CSF, the analysis revealed that in particular the albumin quotient and to a lesser extent age were good predictors for both methyl plant sterols (campesterol and brassicasterol) and for cholesterol, but not for ethyl plant sterols (sitosterol and stigmasterol, Table 2). Furthermore, the presence of APOE4 was a strong covariate in the prediction of campesterol concentrations. In addition, phospho-tau was shown to be a significantly predicting covariate for the ethyl plant sterols and cholesterol in case of $\mathrm{AD}$. Gender, creatinine concentrations and $\mathrm{A} \beta 42$ concentrations were systematically excluded by the backwards linear regression, indicating a rather limited predictive value for the individual plant sterols concentrations.

Subsequently, individual plant sterol concentrations were compared for diagnosis in a general linear model (GLM) after univariate analysis of variance including the covariates which were proven to have a significant predictive value in the backwards linear regression analysis. Herein, it was shown that brassicasterol $(\mathrm{P}<0.001)$ and sitosterol $(\mathrm{P}<0.05)$ were significantly lower in CSF of AD patients compared to the healthy controls (Table 4). When the CSF plant sterol concentrations were related to the corresponding CSF cholesterol concentrations, only brassicasterol remained significantly different between the AD and control group (Table 4).

Plant sterol concentrations in plasma are indistinguishable between AD patients and Healthy controls

In plasma, the presence of APOE4 allele and, to a lesser extent, age were good predictors for campesterol, brassicasterol and sitosterol. For plasma cholesterol, rather gender and the presence of APOE4 were strong predictors (Table 3). Moreover, CSF-A $\beta 42$ concentrations were highly predictive for plasma campesterol, sitosterol and stigmasterol concentrations in the healthy control group only. Comparison of individual plasma plant sterol concentrations by diagnosis in the GLM with univariate analysis of variance including the predicting covariates revealed that none of the plant sterols were significantly different between the healthy control group and the AD patients (Table IV). After correction for plasma cholesterol concentrations, stigmasterol $(\mathrm{P}<0.05)$ was significantly lower in plasma of AD patients. 


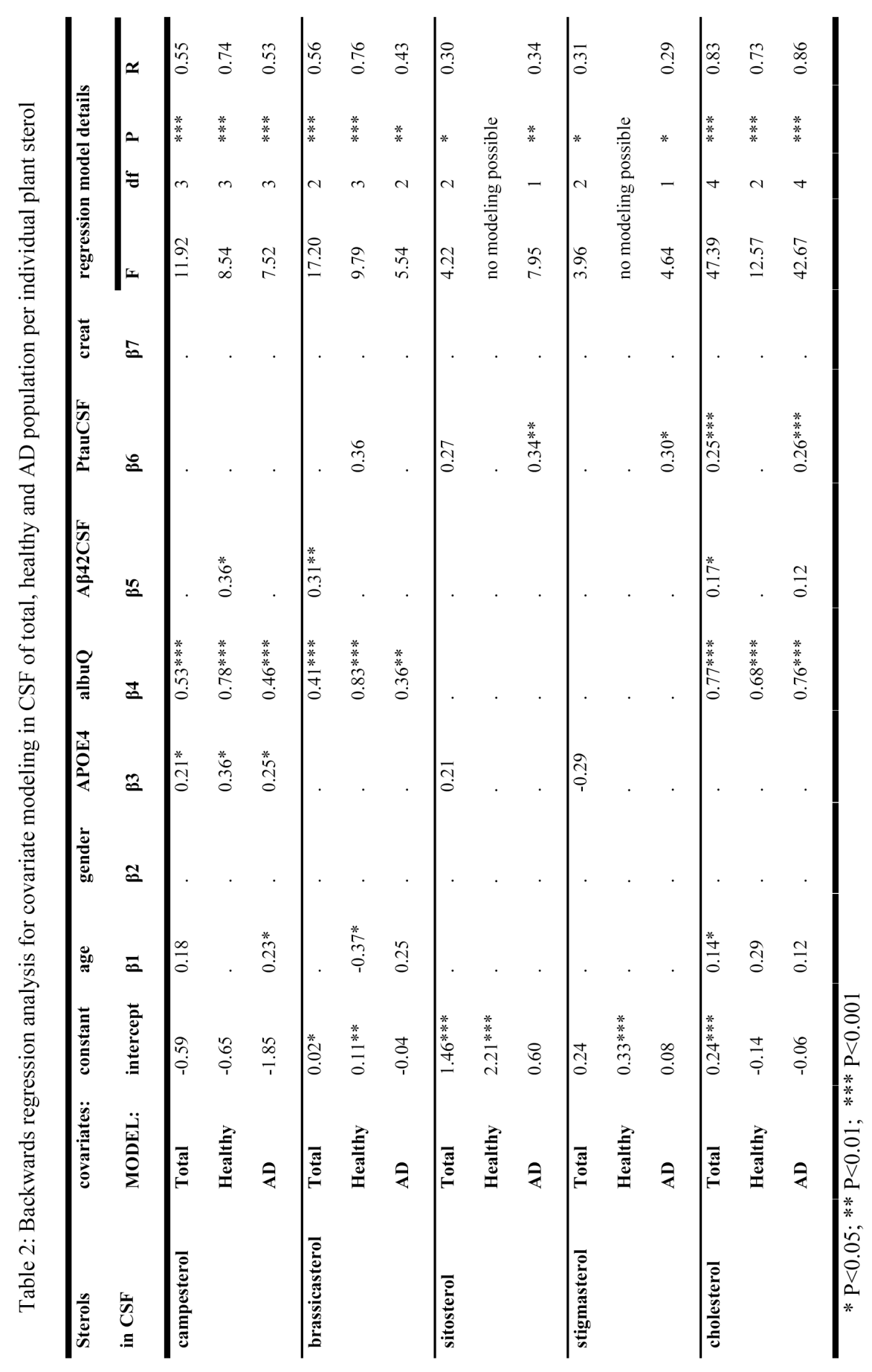




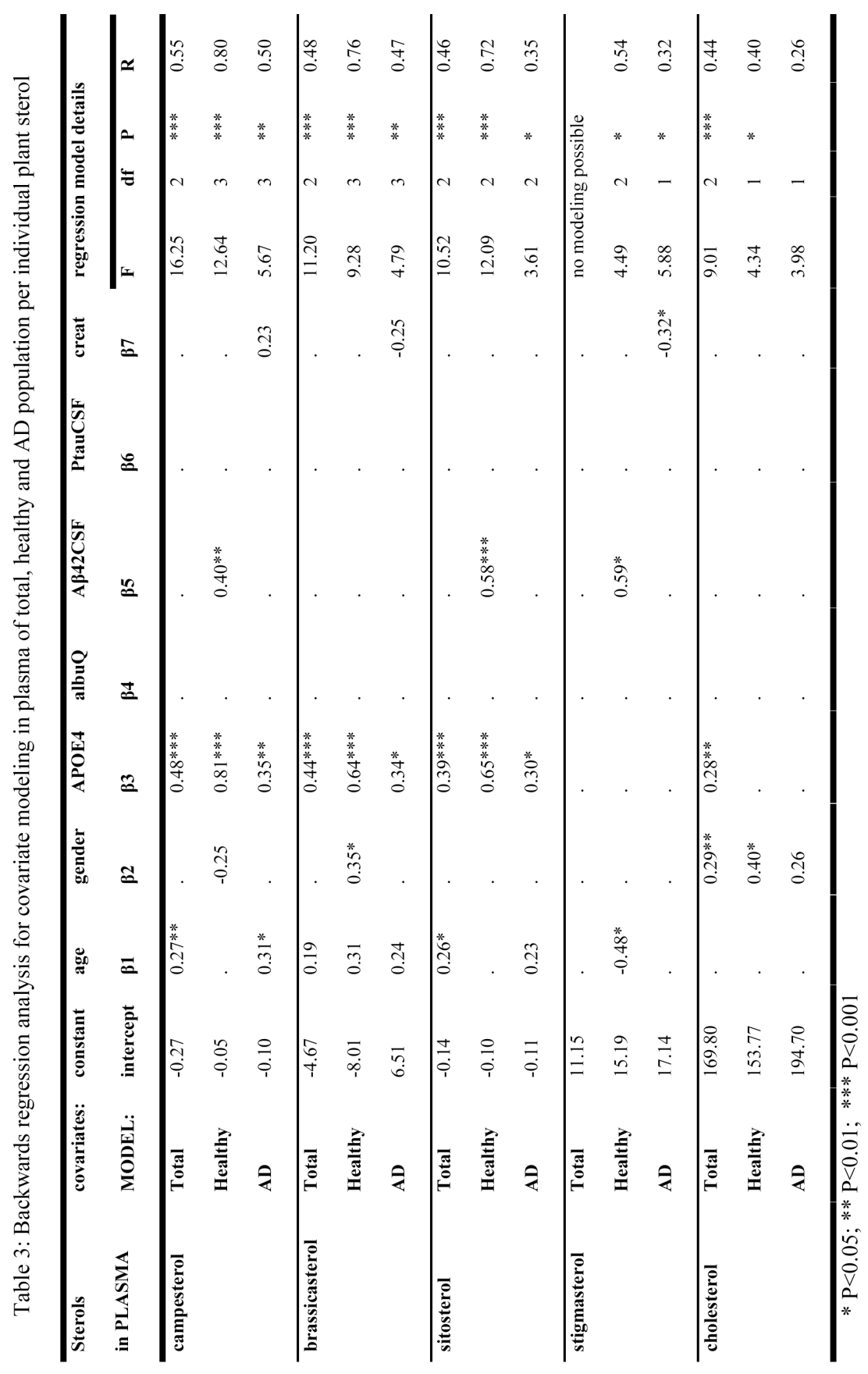




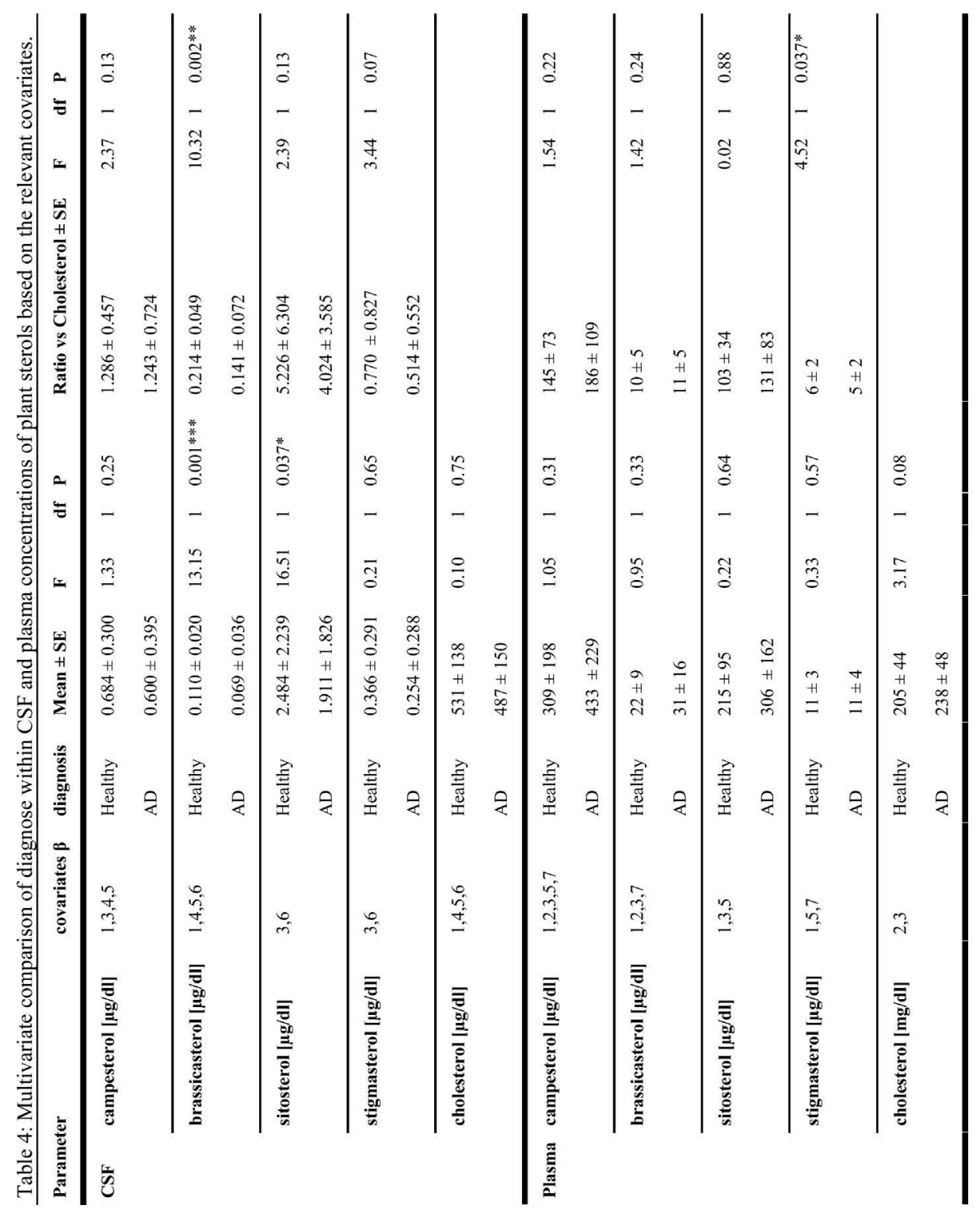

Covariates $\beta$ (1-7) were implied in the GLM univariate ANOVA, based on the integration in the final regression model as generated by backwards linear regression analysis as represented in Table II and III. ( $\beta 1=$ age, $\beta 2=$ gender, $\beta 3=$ possession of APOE4 allele, $\beta 4=$ albumin quotient, $\beta 5=\mathrm{CSFA} \beta 42, \beta 6=$ CSFphospho-tau, $\beta 7=$ plasma creatinine). ${ }^{*} \mathrm{P}<0.05 ; * * \mathrm{P}<0.01 ; * * * \mathrm{P}<0.001$ 


\section{Discussion}

In this study we tested the hypothesis that CSF plant sterols are decreased in $\mathrm{AD}$ patients and therefore may be suitable biomarkers for $\mathrm{AD}$. We found a strong correlation between albumin quotient and methyl plant sterols in CSF. In addition, we showed that the absolute concentrations of brassicasterol and sitosterol were significantly reduced in the $\mathrm{CSF}$ of $\mathrm{AD}$ patients compared to non-demented controls. When corrected for CSF cholesterol concentrations, only brassicasterol remained significantly reduced in CSF of AD patients. In contrast, absolute plant sterol concentrations in the plasma were comparable between the two groups. Our data suggest a plant sterol specific difference in transport across the CSF-brain or CSF-blood barrier in AD patients compared to controls.

So far it has been shown that the brain specific cholesterol metabolite 24(S)hydroxycholesterol is found to be increased in CSF of AD patients, whereas cholesterol precursors were reduced, pointing to an $\mathrm{AD}$ related disturbance in cholesterol turnover [21,22]. Based on their exclusive exogenous nature and the inability to be metabolized into 24-OH-sterols, plant sterols are good markers for sterol flux between the different compartments separated by the BBB, blood-CSF barrier and CSF-brain barrier.

Recently, we reported accumulation of sitosterol and campesterol within the brains of Abcg5-/- mice and of Watanabe rabbits [12, 13]. Due to their relatively low plasma concentrations, not much is known with respect to the $\Delta 22$ plant sterols (stigmasterol and brassicasterol) within the brain and the CSF. Independent of AD pathology, we showed that the albumin quotient was a strong predictor for methyl plant sterol concentrations in the CSF. The albumin ratio served as a measure of blood-CSF barrier function [23, 24]. Although both AD patients and control patients have albumin quotients within the normal range, $\mathrm{AD}$ patients show small but significantly lower albumin quotients. In line herewith, all plant sterols, from which sitosterol and brassicasterol significantly, are reduced in CSF of AD patients, compared to the non-demented controls. These data indicate that the secretion and re-secretion of plant sterols and albumin is depending on the functionality of the blood-CSF and/or CSF-brain barrier. Although plasma concentrations of plant sterols are indistinguishable between $\mathrm{AD}$ patients and non-demented controls, all plant sterol concentrations, in particular sitosterol and brassicasterol, are retrieved less in the CSF of $\mathrm{AD}$ patients. The choroid plexus maintains the vital function of producing up to $90 \%(450-1000 \mathrm{ml} /$ day) of cerebrospinal fluid (CSF). The choroid 
plexus also acts as a selective barrier between blood and CSF to regulate proteins, lipoprotein particles and other essential molecules $[25,26]$. A number of pathological alterations have been observed in the choroid plexus of AD patients affecting the vital functions of the choroid plexus [27, 28]. Patients with a leaky blood-CSF barrier were excluded from the analysis. Previously, a reduced secretory function of the choroid plexus was proven by a reduced folate, vitamin B12, vitamin $\mathrm{E}$ and transthyretin secretion in a group of AD patients [29-33]. Morphological studies further demonstrated fibrosis and basement membrane thickening in choroid plexus of deceased AD patients [28, 34]. This suggests that choroid plexus alterations, leading to an abnormal production of CSF, could be involved in the pathogenesis of late-onset AD. Therefore, a disturbed secretory function of the choroid plexus could at least partially underlie the reduction in CSF plant sterols in AD [28]. Interestingly, phospho-tau in CSF is a strong predictor for the ethyl plant sterols in CSF only in AD patients, supporting a parallel re-secretory deficit. Corroborating previous studies $[35,36]$, we showed that the presence of the APOE4 allele was highly predictive for plant sterol and cholesterol concentrations in the plasma in humans and also in mice $[37,38]$. Furthermore, A $\beta 42$ in CSF was a strong predictor for campesterol, sitosterol and stigmasterol in plasma only in the non-demented group.

Furthermore, sitosterol is the most abundant plant sterol in the CSF. Sitosterol concentrations in CSF within the total population were 3.2 fold higher than CSF campesterol concentrations, whereas plasma campesterol is 1.4 fold higher than plasma sitosterol, resulting in a 4.5 fold higher accumulation of sitosterol in the CSF. In contrast, intestinal campesterol absorption by the enterocytes is 2 fold higher than for sitosterol [39]. In parallel herewith, it was shown that transport of campesterol into murine brains is more efficient than for sitosterol [13]. In nondemented human post mortem hippocampal brain samples $(\mathrm{n}=5$; data not shown) we found $50 \%$ higher campesterol concentrations compared to sitosterol concentrations, indicating a 1.2 fold improved flux for campesterol from serum towards the brain. A similar 7.7 fold higher stigmasterol accumulation compared to brassicasterol was measured in the CSF. Since methyl plant sterols (campesterol and brassicasterol) in comparison to ethyl plant sterols (sitosterol and stigmasterol) are found to be higher in both the brain and the plasma compartment [13] and based on the transport advantages of methyl plant sterols in other cell types, it seems unlikely that increased secretion of ethyl plant sterols from plasma towards the CSF is underlying their higher concentrations. Alterations in plant sterol secretion at the level of the brain-CSF barrier are not expected to be involved in plant sterol CSF 
concentrations since we recently showed that the accumulation of plant sterols in the brain is irreversible, and therefore the secretion out of the brain is expected to be neglected (chapter V). Together, in total population these data indicate a side chain specific and therefore lipophilicity dependent, sterol specific transport across the blood-CFS barrier, with a less efficient secretion/re-secretion of ethyl sterols compared to methyl sterols at the level of choroid plexus, resulting in a net increase in ethyl plant sterols in the CSF.

Consumption of high amounts of plant sterols $(\sim 2.25 \mathrm{~g} /$ day $)$ leads to a reduction of serum and LDL cholesterol concentrations (10-15\%) [40, 41]. Cholesterol loading and depletion in in vitro systems and transgenic mouse models were found to modulate the efficiency to cleave amyloid precursor protein (APP), leading to the production of $\beta$-amyloid-42 (A $\beta 42)$ peptide [42-45]. In parallel, in vitro stimulation of neuronal cells stably over-expressing $\mathrm{APP}_{\text {swe }}$ with plant sterols showed that only sitosterol reduced the processing of APP into $A \beta 40$ and $A \beta 42$ (chapter IV). Therefore, a possibility of an in vivo disease modulating connection between plant sterols and $\mathrm{AD}$ cannot be excluded.

In conclusion, our data demonstrate that the re-secretion of plant sterols from the CSF into the plasma is limited by the complexity of the side-chain, leading to relatively higher CSF sitosterol and stigmasterol concentrations in the total population. Moreover, our data suggest that the secretion of plant sterols from the plasma via the choroid plexus into CSF is reduced in association with $\mathrm{AD}$ pathogenesis. After correction for the CSF cholesterol content, only brassicasterol remained strongly related to $\mathrm{AD}$. Therefore, CSF brassicasterol may be a suitable additional biomarker, besides existing biomarkers for AD such as brain atrophy, tau, phospho-tau en A $\beta 42$.

\section{Acknowledgements}

The project was financially supported by grants from Marie Curie Early Stage Training Fellowship (MEST-CT-2005-02058), Deutsche Forschungsgemeinschaft (KO2327/2-1), the European Community's Seventh Framework Programme (FP7/2007-2013) under grant (agreement $\mathrm{n}^{\circ} 211696$ ), and by the German Federal Ministry for Education and Research within the framework of the Competence Network Dementia (grant: 01GI0422). There are no actual conflicts of interest. 


\section{References}

1. Itoh, N., et al., Large-scale, multicenter study of cerebrospinal fluid tau protein phosphorylated at serine 199 for the antemortem diagnosis of Alzheimer's disease. Ann Neurol, 2001. 50(2): p. 1506.

2. de Leon, M.J., et al., Longitudinal CSF and MRI biomarkers improve the diagnosis of mild cognitive impairment. Neurobiol Aging, 2006. 27(3): p. 394-401.

3. Du, A.T., et al., Different regional patterns of cortical thinning in Alzheimer's disease and frontotemporal dementia. Brain, 2007. 130(Pt 4): p. 1159-66.

4. Goedert, M. and M.G. Spillantini, A century of Alzheimer's disease. Science, 2006. 314(5800): p. 777-81.

5. Hampel, H., et al., Core candidate neurochemical and imaging biomarkers of Alzheimer's disease. Alzheimers Dement, 2008. 4(1): p. 38-48.

6. Shaw, L.M., et al., Cerebrospinal fluid biomarker signature in Alzheimer's disease neuroimaging initiative subjects. Ann Neurol, 2009. 65(4): p. 403-13.

7. Mulder, M., Sterols in the central nervous system. Curr Opin Clin Nutr Metab Care, 2009. 12(2): p. 152-8

8. Dietschy, J.M., S.D. Turley, and D.K. Spady, Role of liver in the maintenance of cholesterol and low density lipoprotein homeostasis in different animal species, including humans. J Lipid Res, 1993. 34(10): p. 1637-59.

9. Segal, M.B., The choroid plexuses and the barriers between the blood and the cerebrospinal fluid. Cell Mol Neurobiol, 2000. 20(2): p. 183-96.

10. Pollak, O.J. and D. Kritchevsky, Sitosterol. Monogr Atheroscler, 1981. 10: p. 1-219.

11. Salen, G., E.H. Ahrens, Jr., and S.M. Grundy, Metabolism of beta-sitosterol in man. J Clin Invest, 1970. 49(5): p. 952-67.

12. Fricke, C.B., et al., Increased plant sterol and stanol levels in brain of Watanabe rabbits fed rapeseed oil derived plant sterol or stanol esters. Br J Nutr, 2007. 98(5): p. 890-9.

13. Jansen, P.J., et al., Dietary plant sterols accumulate in the brain. Biochim Biophys Acta, 2006.

14. Bowman, G.L., et al., Blood-brain barrier impairment in Alzheimer disease: stability and functional significance. Neurology, 2007. 68(21): p. 1809-14.

15. Fratiglioni, L., et al., Incidence of dementia and major subtypes in Europe: A collaborative study of population-based cohorts. Neurologic Diseases in the Elderly Research Group. Neurology, 2000. 54(11 Suppl 5): p. S10-5.

16. Hebert, L.E., et al., Age-specific incidence of Alzheimer's disease in a community population. Jama, 1995. 273(17): p. 1354-9.

17. Lobo, A., et al., Prevalence of dementia and major subtypes in Europe: A collaborative study of population-based cohorts. Neurologic Diseases in the Elderly Research Group. Neurology, 2000. 54(11 Suppl 5): p. S4-9.

18. Andreasen, N., et al., Evaluation of CSF biomarkers for axonal and neuronal degeneration, gliosis, and beta-amyloid metabolism in Alzheimer's disease. J Neurol Neurosurg Psychiatry, 2001. 71(4): p. 557-8.

19. Folstein, M.F., S.E. Folstein, and P.R. McHugh, "Mini-mental state". A practical method for grading the cognitive state of patients for the clinician. J Psychiatr Res, 1975. 12(3): p. 189-98.

20. Teunissen, C.E., et al., Serum cholesterol, precursors and metabolites and cognitive performance in an aging population. Neurobiol Aging, 2003. 24(1): p. 147-55.

21. Kolsch, H., et al., Alterations of cholesterol precursor levels in Alzheimer's disease. Biochim Biophys Acta, 2010.

22. Leoni, V., et al., Are the CSF levels of 24S-hydroxycholesterol a sensitive biomarker for mild cognitive impairment? Neurosci Lett, 2006. 397(1-2): p. 83-7.

23. Blennow, K., et al., Protein analysis in cerebrospinal fluid. II. Reference values derived from healthy individuals 18-88 years of age. Eur Neurol, 1993. 33(2): p. 129-33.

24. Tibbling, G., H. Link, and S. Ohman, Principles of albumin and IgG analyses in neurological disorders. I. Establishment of reference values. Scand J Clin Lab Invest, 1977. 37(5): p. 385-90.

25. Spector, R. and C.E. Johanson, The mammalian choroid plexus. Sci Am, 1989. 261(5): p. 68-74. 
26. Fujiyoshi, M., et al., 24S-hydroxycholesterol induces cholesterol release from choroid plexus epithelial cells in an apical- and apoE isoform-dependent manner concomitantly with the induction of ABCA1 and ABCG1 expression. J Neurochem, 2007. 100(4): p. 968-78.

27. Wen, G.Y., H.M. Wisniewski, and R.J. Kascsak, Biondi ring tangles in the choroid plexus of Alzheimer's disease and normal aging brains: a quantitative study. Brain Res, 1999. 832(1-2): p. 40-6.

28. Serot, J.M., et al., Morphological alterations of the choroid plexus in late-onset Alzheimer's disease. Acta Neuropathol, 2000. 99(2): p. 105-8.

29. Hagnelius, N.O., L.O. Wahlund, and T.K. Nilsson, CSF/serum folate gradient: physiology and determinants with special reference to dementia. Dement Geriatr Cogn Disord, 2008. 25(6): p. 516-23.

30. Ikeda, T., et al., Vitamin B12 levels in serum and cerebrospinal fluid of people with Alzheimer's disease. Acta Psychiatr Scand, 1990. 82(4): p. 327-9.

31. Jimenez-Jimenez, F.J., et al., Cerebrospinal fluid levels of alpha-tocopherol (vitamin E) in Alzheimer's disease. J Neural Transm, 1997. 104(6-7): p. 703-10.

32. Riisoen, H., Reduced prealbumin (transthyretin) in CSF of severely demented patients with Alzheimer's disease. Acta Neurol Scand, 1988. 78(6): p. 455-9.

33. Serot, J.M., et al., Cerebrospinal fluid transthyretin: aging and late onset Alzheimer's disease. J Neurol Neurosurg Psychiatry, 1997. 63(4): p. 506-8.

34. Jellinger, K. and A. Rett, Agyria-pachygyria (lissencephaly syndrome). Neuropadiatrie, 1976. 7(1): p. 66-91.

35. Jansen, P.J., et al., Absence of ApoE Upregulates Murine Brain ApoD and ABCA1 Levels, But Does Not Affect Brain Sterol Levels, While Human ApoE3 and Human ApoE4 Upregulate Brain Cholesterol Precursor Levels. J Alzheimers Dis, 2009.

36. Kempen, H.J., et al., Plasma levels of lathosterol and phytosterols in relation to age, sex, anthropometric parameters, plasma lipids, and apolipoprotein E phenotype, in 160 Dutch families. Metabolism, 1991. 40(6): p. 604-11.

37. Geelen, A., et al., Apolipoprotein E polymorphism and serum lipid response to plant sterols in humans. Eur J Clin Invest, 2002. 32(10): p. 738-42.

38. Hallikainen, M.A., et al., Comparison of the effects of plant sterol ester and plant stanol esterenriched margarines in lowering serum cholesterol concentrations in hypercholesterolaemic subjects on a low-fat diet. Eur J Clin Nutr, 2000. 54(9): p. 715-25.

39. Heinemann, T., G. Axtmann, and K. von Bergmann, Comparison of intestinal absorption of cholesterol with different plant sterols in man. Eur J Clin Invest, 1993. 23(12): p. 827-31.

40. Miettinen, T.A., et al., Reduction of serum cholesterol with sitostanol-ester margarine in a mildly hypercholesterolemic population. N Engl J Med, 1995. 333(20): p. 1308-12.

41. Thompson, G.R. and S.M. Grundy, History and development of plant sterol and stanol esters for cholesterol-lowering purposes. Am J Cardiol, 2005. 96(1A): p. 3D-9D.

42. Wahrle, S., et al., Cholesterol-dependent gamma-secretase activity in buoyant cholesterol-rich membrane microdomains. Neurobiol Dis, 2002. 9(1): p. 11-23.

43. Hayashi, H., et al., Cholesterol is increased in the exofacial leaflet of synaptic plasma membranes of human apolipoprotein E4 knock-in mice. Neuroreport, 2002. 13(4): p. 383-6.

44. Guardia-Laguarta, C., et al., Mild cholesterol depletion reduces amyloid-beta production by impairing APP trafficking to the cell surface. J Neurochem, 2009. 110(1): p. 220-30.

45. Vetrivel, K.S. and G. Thinakaran, Membrane rafts in Alzheimer's disease beta-amyloid production. Biochim Biophys Acta, 2010. 



\section{Chapter VII}

\section{General Discussion}


Alterations in peripheral and central cholesterol metabolism are closely associated with the development and progression of $\mathrm{AD}$. In this thesis, we addressed the question whether the modulation of the brain sterol metabolism could influence AD. So far, based on mutations in genes causing FAD, several animal models are generated and available to study AD. In contrast to the commonly used AD-models that overexpress mutants of the human APP specifically several-fold in neurons [1], APPSLXPS1mut mice carry a humanized (knock-in) APP sequence, that contains both the Swedish and the London mutation under the control of the endogenous murine APP promoter [2]. Therefore, expression of APPSL is not restricted to neurons but comparable to that of murine-APP, and the levels of APPSL are in the physiological range. When crossed with PS1M146L-transgenic mice, amyloid is deposited predominantly in the cortex and in the hippocampus from the age of 6-9 months onwards with a steep increase from the age of 18 months [2]. In this APPSLXPS1mut mouse model, we found an increased cholesterol turnover, following the pathological AD manifestation. Our results pointed towards a secondary LXR activation in an attempt to rescue the brain from AD associated pathology. The nuclear receptor, LXR, is a master regulator in peripheral and central cholesterol turnover. Therefore, we subsequently administered a synthetic LXR agonist T0901317 to aged APPSLxPS1mut mice and found a significant memory improvement, however, independent of A $\beta$ deposition. Since plant sterols are described to be naturally occurring LXR agonists able to accumulate within the brain, we hypothesized a disease modulating influence of plant sterols on AD. Therefore we first studied the possibility of a concentration dependent dietary derived cerebral plant sterol accumulation within the brain. We found an irreversible accumulation of plant sterols within mice brains, without affecting normal brain functions. However in relation to $\mathrm{AD}$, we found a significant in vitro reduction in $A \beta$ processing upon administering the specific plant sterol sitosterol. In addition, a reduction in CSF sitosterol and brassicasterol were found to be hallmarks for $\mathrm{AD}$. Together, we believe that this thesis provides a strong basis to further study the in vivo potential of plant sterols to modulate AD. Dietary intervention in the treatment and prevention of $\mathrm{AD}$ provides important future perspectives.

The production and aggregation of $\mathrm{A} \beta$ is considered a key event in neuronal dysfunction and in early tissue impairment in AD. Any factor modulating APP processing and $\mathrm{A} \beta$ deposition can be of high importance in $\mathrm{AD}$ pathogenesis [3]. Chapter I focuses on the role of cholesterol in the fate of $A \beta$. 
Epidemiological studies indicate a strong association between high mid-life plasma cholesterol concentrations and the risk of sporadic LOAD [4-8]. In parallel, the cholesterol synthesis inhibitors that lead to reduced circulating cholesterol concentrations also reduce the risk of $\mathrm{AD}$ development $[9,10]$. However, peripheral cholesterol is generally assumed not to cross the BBB [11]. An overview of retrospective studies on the relationship between the use of statins and a reduction in risk of $\mathrm{AD}$ development did not reveal a uniform outcome [12]. Moreover, irrespective of their potential to cross the $\mathrm{BBB}$, positive associations were found, indicating a brain cholesterol independent association. Together, the risk modulating influence of peripheral cholesterol concentrations on the development of $\mathrm{AD}$ are assumed to be via indirect paths. Accelerated brain vessel atherosclerosis, a reduction of cerebral blood circulation and a reduced CNS oxygen and glucose supply upon increased peripheral plasma cholesterol concentrations resulted in cerebral parenchymal stress, increased susceptibility to $\mathrm{AD}$ damage and a reduced regeneration capacity of the brain $[13,14]$. These data provide at least in part a rationale explanation for high plasma cholesterol as indirect risk factor for $\mathrm{AD}[3]$.

Next, we investigated whether alterations in the brain cholesterol homeostasis are cause or consequence in the disease pathology (Chapter II). Controversial data and hypothesises exist concerning the etiologic role of cerebral cholesterol in the development of AD. In a first model it is postulated that high neuronal membrane cholesterol is in favor of $\mathrm{AD}$ development. Increased neuronal membrane cholesterol would induce clustering of APP with $\beta / \gamma$-secretase complexes and consequently result in an improved APP processing into the toxic A $\beta$ fragments and AD pathology [15]. Corroborating this hypothesis, the reduction in neuronal cholesterol resulted in an impaired transport of APP to the neuronal membrane, a disturbed APP internalization, a reduced $\beta / \gamma$-cleavage of APP and increased ADAM-10 expression [16-19]. However, most of these studies were based on unphysiological membrane cholesterol depletion upon methyl $\beta$-cyclodextrin administration, therewith inhibiting APP endocytosis, required in APP processing by $\beta / \gamma$-secretases. In contrast, other groups reported a role for high membrane cholesterol in the prevention of $A \beta$ generation. In this second model, a reduction of membrane cholesterol is associated with a translocation of $\beta$-secretase from lipid raft domains towards the APP-rich phospholipid membrane domains [15]. Therefore, membrane cholesterol is essential in order to prevent contact between APP and $\beta / \gamma$-secretases. Consequently, it was found that mild reduction of membrane cholesterol and subsequent lipid raft depletion resulted in an increased $\mathrm{A} \beta$ generation [20-26]. In line with this last hypothesis, it has been shown that AD 
patients have moderately decreased hippocampal membrane cholesterol contents [27]. On the other hand, the rate limiting enzyme in cholesterol synthesis, HMGCR, is negatively regulated by $\mathrm{A} \beta 40$ pointing to a tight communication [28]. Yet, despite elaborated theories and obvious associations between cholesterol and APP, it is not clear whether alterations in the brain cholesterol homeostasis are preceding or following disease pathology.

In our study, we therefore investigated brain cholesterol homeostasis in the APPSLXPS1mut AD mouse model before the onset of the pathology (9 months) and upon strong presence of A $\beta$ pathology (21 months) [2]. The APPSLxPS1mut mice displayed age-related alterations in their brain sterol profiles and in the expression of cholesterol metabolism-related genes in comparison with wild-type mice. Brain levels of cholesterol precursors, in particular desmosterol and metabolite 24SOHcholesterol were found to be increased in particular in the 21-month old APPSLXPS1mut mice, in parallel with the increasing amyloid deposition [2]. Astrocyte dependent desmosterol upregulation and neuronal 24(S)-OHcholesterol upregulation are driving an LXR mediated cholesterol turnover [29-31]. In addition, LXR-responsive genes (Abcal and Apoc1) were significantly upregulated. Together our data suggest an overall enhanced cholesterol turnover in the brains of the APPSLXPS1mut mice, in particular in the hippocampus, being secondary to the development of $\mathrm{AD}$ pathology. Therefore, the data indicate that brain cholesterol metabolism is altered as a consequence of the ongoing neuropathology induced by the expression of the APPSL and/or PS1M146L mutants in this murine AD model. We consequently concluded that increased brain cholesterol turnover may be a rescue mechanism possibly to remove the toxic $\mathrm{A} \beta$ peptides and cope with the pathology. However, we do not exclude a causal role for cholesterol in AD.

Based on the experiments performed in Chapter II, we hypothesized that enhancement of the cerebral cholesterol turnover by administration of a synthetic LXR agonist, T0901317, may result in a reduction of A $\beta$ deposition (Chapter III). T0901317 was administered during the steep onset of the pathology progress in the APPSLXPS1mut mice for a period of 10 weeks (21-23 months). LXR activation had been shown in vitro to reduce AD pathology [32-34]. We therefore hypothesized that LXR activation would reduce AD pathology in parallel with a cognitive improvement in aged APPSLXPS1mut mice. We demonstrated that long-term pharmacological LXR-activation by T0901317 partly restores/prevents the impaired object and spatial memory in aged APPSLxPS1mut mice with major A $\beta$ deposition in their brain. In line with previous observations, T0901317 treatment up regulated concentrations of cholesterol precursors, lathosterol, lanosterol and desmosterol, in 
all brain regions examined, indicating an enhanced cholesterol turnover in the brain [35]. Abca1, Apoe and Abcg1 may be involved in the enhanced cholesterol flux, since their expression was increased upon T0901317 treatment. In contrast with our hypothesis, the enhanced cholesterol turnover in brain was not accompanied by a decrease in hippocampal and cortex $A \beta$ plaque load. Our data suggest that a reduction in $\mathrm{A} \beta$ plaque load is not required for improved memory functions in this model, but that an enhanced cholesterol turnover in the brain may be involved. Although T0901317 treatment also upregulated levels of cholesterol precursors and the expression of LXR-target genes in brain of $\mathrm{C} 57 \mathrm{BL} / 6 \mathrm{NCrl}$ mice, it did not further improve their memory functions.

Jiang and coworkers [36] showed that LXR activation resulted in an improved lipidation status of apoE, mainly due to an upregulation of ABCA1 in astrocytes. Consequently, they show that lipidated apoE promotes both microglial intracellular and extracellular interstitial degradation of $A \beta$ in an isoform $(E 2>E 3>E 4)$ and lipidation status dependent manner. In addition, they show that short term (6 days) administration of the synthetic LXR-agonists GW3965 and T0901317 to 20 weeksold pre-plaque Tg2576 mice improved memory performance [36, 37]. Furthermore, 4 months of GW3965 administration to 12 months old Tg2576 is described to reduce total plaque number with 50\% [36]. LXR activation reduced the $A \beta_{40}$ and $\mathrm{A} \beta_{42}$ levels both in vitro and in vivo in $\mathrm{AD}$-models [32, 33, 37]. Moreover, T0901317 was reported to decrease the neuronal amyloid production independent of cholesterol efflux [33]. In contrast herewith, in our experiments, no significant differences in $\mathrm{A} \beta$ deposition was detected in hippocampus or cortex of the APPSLxPS1mut mice after T0901317 administration to aged APPSLxPS1mut mice. This indicates that a reduction in $A \beta$ plaque load is not essential for improvement of memory functions. APPSLxPS1mut mice display a steep increase in $\mathrm{A} \beta$ plaque deposition from 18 months on. LXR activation may prevent the deposition of $A \beta$, but not reduce the already deposited $A \beta$. Therefore, the possibility remains that administration of T0901317 starting at the pre-plaque stage, would have prevented $\mathrm{A} \beta$ deposition in the APPSLxPS1mut mice. However, dissociation between memory performance and $A \beta$ plaque load or levels has been reported by others as well $[38,39]$.

LXR activation results in depletion of cholesterol from membrane lipid rafts and consequently has been reported to decrease the production of amyloid [40]. It has been suggested that the deposition of $A \beta$ is prevented by increased interstitial concentrations of HDL-like particles by binding A $\beta$ thereby preventing its deposition and/or by directing it to glial cells for degradation [41]. The increased 
levels of cholesterol precursors, together with the upregulation of the expression of Abca1, Abcg1 and Apoe by T0901317 suggest that the levels of interstitial HDLlike particles may increase due to an enhanced secretion of cholesterol. The expression of Abca1, Abcg1 and Apoe were increased by T0901317 treatment in all brain regions with the exception of the cortex of the APPSLxPS1mut mice, where the largest number of plaques is found. This suggests that the altered processing of APP and/or the deposition of A $\beta$ interferes with the LXR-pathway in the cortex. All together, we can conclude that LXR agonists can have treating and prophylactic potential in $\mathrm{AD}$. However, so far, the undesirable contra-indication of LXR agonists is the coinciding liver lipogenesis, which leads to hypertriglyceridemia and liver steatosis. LXRs, as part of the nuclear receptor family, consist of two isoforms, namely: $\mathrm{LXR} \alpha(\mathrm{NR} 1 \mathrm{H} 3)$ and $\mathrm{LXR} \beta(\mathrm{NR} 1 \mathrm{H} 2)$. $\mathrm{LXR} \alpha$ is mainly expressed in liver, adrenal glands, intestine, adipose tissue and macrophages, whereas LXR $\beta$ is expressed ubiquitously [42]. LXR $\alpha$ and LXR $\beta$ are both present in the brain and thought to be involved in the regulation of brain cholesterol homeostasis [43]. To overcome the liver steatosis, an LXR $\beta$ specific agonist is desirable [44]. The peripheral synthetic LXR agonist WAY-252623 is described to have a preference for LXR $\beta$ activation and to overcome long term plasma triglyceride elevation [45]. So far no central effects of the WAY-252623 compound are described.

Interestingly, plant sterols have been found to activate LXRs, although the literature is inconsistent. Yang et al. showed in a CHO-7 cell line, using reporter gene assay that stigmasterol is a potent LXR agonist [46]. In addition, Plat et al., demonstrated in a cell free system that sitosterol, campesterol and fucosterol have an LXR agonist activity [47]. However, Kaneko et al. could not confirm LXR agonist activity in a human embryonic kidney cell line (HEK293) using a LXR-GAL4-chimeric receptor / GAL4 responsive luciferase reporter assay for any of the naturally occurring phytosterols tested (sitosterol, campesterol, brassicasterol, fucosterol, sitostanol, and campestanol) or ergosterol [48]. Together these data suggest a cell-type or tissue specific co-factor recruitment, determining differences in LXR agonizing potential for the individual phytosterols. Recently, it became clear that plant sterols can accumulate within murine brains [49]. In parallel a sitosterol specific upregulation of LXR responsive genes in a human astrocytoma, though not in a human neuroblastoma cell line was demonstrated [49]. Since astrocytes are the most important cholesterol synthesizing cells in the brain, beneficial effects due to an LXR dependent increased cholesterol turnover upon plant sterols supplementation in $\mathrm{AD}$ can be hypothesized. Therefore, we questioned in Chapter IV whether this accumulation of plant sterols in the brain would be reversible and 
whether plant sterol accumulation can modulate cholesterol related pathology in Alzheimer's disease (AD).

In this chapter we demonstrated for the first time that increased dietary intake of plant sterols resulted in a concentration dependent irreversible accumulation of plant sterols in the brain. The flux of campesterol across the BBB, was both in vitro and in vivo significantly more efficient than for sitosterol. In addition, beyond the $\mathrm{BBB}$, we showed both in vitro and in vivo that plant sterols preferentially accumulated within lipid rafts of brain cells. Moreover, plant sterols have been shown to reduce the molecular order in membranes by interacting less efficiently with saturated phospholipids compared to cholesterol as required in the formation of compact, liquid ordered lipid rafts and therefore alter the membrane fluidity [50, 51]. It was demonstrated that the magnitude of the foregoing effect depends on the geometry of the sterol molecule that is determined by the structure of its side chain (cholesterol $>>$ campesterol $>$ sitosterol $>$ stigmasterol) [50]. Mild membrane cholesterol reduction impacts the cleavage of APP upstream of $\gamma$-secretase and is mediated by changes in APP trafficking and partitioning into lipid rafts [16]. Moreover, processing of APP into A $\beta$ is requiring cholesterol rich lipid raft microdomains [52]. Plant sterols are known to be organized in lipid rafts within plant cell membranes [53-55]. Therefore, based on the lipid raft accumulation and LXR agonizing potential, we hypothesized that plant sterols could modulate AD pathology by interfering with the membrane cholesterol metabolism in an in vitro setup. However, only upon sitosterol stimulation, APP cleavage into A $\beta 40$ and A $\beta 42$ peptides was significantly lowered in neuronal cell lines due to a diminished APP glycosylation. Since only sitosterol and not campesterol and/or stigmasterol reduced the cleavage of APP, it is unlikely that the alteration of lipid raft rigidity, but rather a sitosterol specific effect is involved in the underlying mechanisms. These data implicate a potential for plant sterols in future AD treatment by dietary supplementation.

Based on the irreversible character of the plant sterols accumulation in the brain described in chapter IV and the increasing application of plant sterol enriched food spreads in the prevention of atherosclerosis [56], we questioned in Chapter $\mathbf{V}$ whether plant sterols could modulate brain functions in a "healthy" brain. We therefore compared cognitive, motor, mood and anxiety related behavior between an Abcg5 knockout model and their respective littermates. Abcg5-/- mice are featured by strongly increased serum and brain plant sterol concentrations, due to an intestinal an liver plant sterol efflux disturbance [49, 57]. Within the brains of Abcg5-/- mice we showed a strong accumulation of plant sterols in the 
hippocampus and the cortex, but even stronger in the cerebellum. The accumulation of plant sterols in the brain only had limited consequences on brain cholesterol metabolism in specific brain regions. Therefore, it is of major importance that we demonstrated that strongly elevated serum and brain plant sterol concentrations in an Abcg5-/- mouse model did not result in an overt negative phenotype in behavioral, e.g. learning and memory, and anxiety and mood in the applied behavioral tasks. An exception was increased swimming speed in the MWEM in the Abcg5-/- mice in comparison with the Abcg5+/+ mice. Further research on the consequences of plant sterol administration in human neurodegenerative disease with cognitive decline is required.

In Chapter VI, we investigated whether plant sterol concentrations in CSF and plasma correlated with AD pathology. Female gender, high mid-life plasma cholesterol, decreased CSF A $\beta 42$ and increased CSF phosphorylated-tau are all described to be risk factors of AD [58-63]. In addition, an impaired blood-CSF barrier permeability at the level of the choroid plexus was found to be a risk factor. Based on the solely exogenous nature of plant sterols in the CSF and the impaired choroid plexus function in $\mathrm{AD}$, we hypothesize that plant sterol concentrations in CSF are reduced in AD pathology and therefore can act biomarkers in AD. CSF and plasma plant sterol concentration were compared between a group of diagnosed $\mathrm{AD}$ patients and an age matched control group with age, gender, apoE, albumin quotient, $\mathrm{A} \beta 42$ and phospho-tau as covariates. We found that the absolute concentrations of brassicasterol and sitosterol were significantly reduced in the CSF of $\mathrm{AD}$ patients as compared to non-demented controls. In contrast, absolute plant sterol concentrations in the plasma were comparable between the two groups. Our data suggest a plant sterol specific difference in transport across the CSF-brain or CSF-blood barrier in AD patients as compared to controls. We demonstrated that the re-secretion of plant sterols from the CSF into the plasma is limited by the complexity of the side-chain, leading to relatively higher CSF sitosterol and stigmasterol concentrations in CSF of the total population. Moreover, our data suggest that the secretion of plant sterols from the plasma via the choroid plexus into CSF is reduced in association with $\mathrm{AD}$ pathogenesis. After correction for the CSF cholesterol content, only brassicasterol remained strongly related to AD. Since plasma brassicasterol concentrations are shown to be hardly modifiable, CSF brassicasterol may be a suitable and sturdy additional biomarker, besides tau, Phospho-tau and A $\beta 42$ in AD. 


\section{Conclusions}

In conclusion, in this thesis we demonstrated that alterations in brain cholesterol turnover can be secondary to the AD pathology onset in the APPSLxPS1mut mouse model. In addition, upon synthetic activation of the LXR regulated brain cholesterol turnover, the cognitive decline was partially reversed in aged APPSLXPS1mut mice. Consequently, we deduct from our data that increased cerebral cholesterol turnover may be involved in rescuing the brain from $A \beta$ mediated toxicity on cognitive performances in AD. Furthermore, we found that the dietary available, naturally occurring LXR agonists, plant sterols, accumulated irreversible in mice brains upon short term dietary supplementation. In vitro, we found a reduction in APP cleavage upon sitosterol stimulation only of an $\mathrm{APP}_{\text {swe }}$ overexpressing neuronal cell line. In parallel to LXR activation in non-AD mice, increased cerebral accumulation of plant sterols did not further improve cognitive performances in the Abcg5-/- mice. Together these data indicate the need for further animal experiments concerning the potential therapeutical role of plant sterols in AD.

Furthermore, we showed significantly reduced sitosterol and brassicasterol concentrations in CSF of AD patients, compared to age and gender matched control patients with comparable plasma plant sterol concentrations. Besides phospho-tau and $\mathrm{A} \beta_{42}$, in particular brassicasterol appears to be a sturdy biomarker in $\mathrm{AD}$. Together, we conclude that there is a need for a prospective study addressing long term dietary supplementation of plant sterols in the prevention of AD. However, since the administration of plant sterols in the prevention of atherosclerosis is a current topic of debate [64], the role of plant sterols in the prevention of atherosclerosis should first be clarified. So far, hard end-point studies are lacking, insights in potential underlying molecular mechanisms are unclear and an increasing amount of adverse events upon plant sterol administration in animal studies has been reported [56, 64-66].

In conclusion, we believe that this thesis provides a strong basis to further study the in vivo potential of brain sterol metabolism modulation in the treatment and prevention of $\mathrm{AD}$. Dietary intervention may herein provide important future perspective. 


\section{References}

1. Hsiao, K., et al., Correlative memory deficits, Abeta elevation, and amyloid plaques in transgenic mice. Science, 1996. 274(5284): p. 99-102.

2. Kohler, C., et al., Alzheimer's disease-like neuropathology of gene-targeted APP-SLxPS1mut mice expressing the amyloid precursor protein at endogenous levels. Neurobiol Dis, 2005. 20(2): p. 528-40.

3. Stefani, M. and G. Liguri, Cholesterol in Alzheimer's disease: unresolved questions. Curr Alzheimer Res, 2009. 6(1): p. 15-29.

4. Jarvik, G.P., et al., Interactions of apolipoprotein E genotype, total cholesterol level, age, and sex in prediction of Alzheimer's disease: a case-control study. Neurology, 1995. 45(6): p. 1092-6.

5. Kivipelto, M., et al., Apolipoprotein E epsilon4 allele, elevated midlife total cholesterol level, and high midlife systolic blood pressure are independent risk factors for late-life Alzheimer disease. Ann Intern Med, 2002. 137(3): p. 149-55.

6. Notkola, I.L., et al., Serum total cholesterol, apolipoprotein E epsilon 4 allele, and Alzheimer's disease. Neuroepidemiology, 1998. 17(1): p. 14-20.

7. Whitmer, R.A., et al., Midlife cardiovascular risk factors and risk of dementia in late life. Neurology, 2005. 64(2): p. 277-81.

8. Pappolla, M.A., et al., Mild hypercholesterolemia is an early risk factor for the development of Alzheimer amyloid pathology. Neurology, 2003. 61(2): p. 199-205.

9. Wolozin, B., et al., Decreased prevalence of Alzheimer disease associated with 3-hydroxy-3methyglutaryl coenzyme A reductase inhibitors. Arch Neurol, 2000. 57(10): p. 1439-43.

10. Zandi, P.P., et al., Do statins reduce risk of incident dementia and Alzheimer disease? The Cache County Study. Arch Gen Psychiatry, 2005. 62(2): p. 217-24.

11. Dietschy, J.M. and S.D. Turley, Cholesterol metabolism in the brain. Curr Opin Lipidol, 2001. 12(2): p. 105-12.

12. Shobab, L.A., G.Y. Hsiung, and H.H. Feldman, Cholesterol in Alzheimer's disease. Lancet Neurol, 2005. 4(12): p. 841-52.

13. Beach, T.G., et al., Circle of Willis atherosclerosis: association with Alzheimer's disease, neuritic plaques and neurofibrillary tangles. Acta Neuropathol, 2007. 113(1): p. 13-21.

14. Ledesma, M.D. and C.G. Dotti, The conflicting role of brain cholesterol in Alzheimer's disease: lessons from the brain plasminogen system. Biochem Soc Symp, 2005(72): p. 129-38.

15. Ledesma, M.D. and C.G. Dotti, Amyloid excess in Alzheimer's disease: what is cholesterol to be blamed for? FEBS Lett, 2006. 580(23): p. 5525-32.

16. Guardia-Laguarta, C., et al., Mild cholesterol depletion reduces amyloid-beta production by impairing APP trafficking to the cell surface. J Neurochem, 2009. 110(1): p. 220-30.

17. Kojro, E., et al., Low cholesterol stimulates the nonamyloidogenic pathway by its effect on the alpha -secretase ADAM 10. Proc Natl Acad Sci U S A, 2001. 98(10): p. 5815-20.

18. Simons, M., et al., Cholesterol depletion inhibits the generation of beta-amyloid in hippocampal neurons. Proc Natl Acad Sci U S A, 1998. 95(11): p. 6460-4.

19. Wahrle, S., et al., Cholesterol-dependent gamma-secretase activity in buoyant cholesterol-rich membrane microdomains. Neurobiol Dis, 2002. 9(1): p. 11-23.

20. Abad-Rodriguez, J., et al., Neuronal membrane cholesterol loss enhances amyloid peptide generation. J Cell Biol, 2004. 167(5): p. 953-60.

21. Arispe, N. and M. Doh, Plasma membrane cholesterol controls the cytotoxicity of Alzheimer's disease AbetaP (1-40) and (1-42) peptides. Faseb J, 2002. 16(12): p. 1526-36.

22. Cecchi, C., et al., Seladin-1/DHCR24 protects neuroblastoma cells against Abeta toxicity by increasing membrane cholesterol content. J Cell Mol Med, 2008. 12(5B): p. 1990-2002. 
23. Crameri, A., et al., The role of seladin-1/DHCR24 in cholesterol biosynthesis, APP processing and Abeta generation in vivo. Embo J, 2006. 25(2): p. 432-43.

24. Devanathan, S., et al., Effects of sphingomyelin, cholesterol and zinc ions on the binding, insertion and aggregation of the amyloid Abeta(1-40) peptide in solid-supported lipid bilayers. Febs J, 2006. 273(7): p. 1389-402.

25. Molander-Melin, M., et al., Structural membrane alterations in Alzheimer brains found to be associated with regional disease development; increased density of gangliosides GM1 and GM2 and loss of cholesterol in detergent-resistant membrane domains. J Neurochem, 2005. 92(1): p. 171-82.

26. Tamboli, I.Y., et al., Inhibition of glycosphingolipid biosynthesis reduces secretion of the betaamyloid precursor protein and amyloid beta-peptide. J Biol Chem, 2005. 280(30): p. 28110-7.

27. Ledesma, M.D., et al., Raft disorganization leads to reduced plasmin activity in Alzheimer's disease brains. EMBO Rep, 2003. 4(12): p. 1190-6.

28. Grimm, M.O., et al., Regulation of cholesterol and sphingomyelin metabolism by amyloid-beta and presenilin. Nat Cell Biol, 2005. 7(11): p. 1118-23.

29. Yang, C., et al., Sterol intermediates from cholesterol biosynthetic pathway as liver $X$ receptor ligands. J Biol Chem, 2006. 281(38): p. 27816-26.

30. Nieweg, K., H. Schaller, and F.W. Pfrieger, Marked differences in cholesterol synthesis between neurons and glial cells from postnatal rats. J Neurochem, 2009. 109(1): p. 125-34.

31. Abildayeva, K., et al., 24(S)-Hydroxycholesterol Participates in a Liver X Receptor-controlled Pathway in Astrocytes That Regulates Apolipoprotein E-mediated Cholesterol Efflux. J Biol Chem, 2006. 281(18): p. 12799-12808.

32. Koldamova, R.P., et al., The liver X receptor ligand T0901317 decreases amyloid beta production in vitro and in a mouse model of Alzheimer's disease. J Biol Chem, 2005. 280(6): p. 4079-88.

33. Sun, Y., et al., Expression of liver X receptor target genes decreases cellular amyloid beta peptide secretion. J Biol Chem, 2003. 278(30): p. 27688-94.

34. Zelcer, N., et al., Attenuation of neuroinflammation and Alzheimer's disease pathology by liver $x$ receptors. Proc Natl Acad Sci U S A, 2007. 104(25): p. 10601-6.

35. Repa, J.J., et al., Liver $X$ receptor activation enhances cholesterol loss from the brain, decreases neuroinflammation, and increases survival of the NPC1 mouse. J Neurosci, 2007. 27(52): p. 14470-80.

36. Jiang, Q., et al., ApoE promotes the proteolytic degradation of Abeta. Neuron, 2008. 58(5): p. 68193.

37. Riddell, D.R., et al., The LXR agonist TO901317 selectively lowers hippocampal Abeta42 and improves memory in the Tg2576 mouse model of Alzheimer's disease. Mol Cell Neurosci, 2007. 34(4): p. 621-8.

38. McLean, C.A., et al., Soluble pool of Abeta amyloid as a determinant of severity of neurodegeneration in Alzheimer's disease. Ann Neurol, 1999. 46(6): p. 860-6.

39. Snowdon, D.A., Aging and Alzheimer's disease: lessons from the Nun Study. Gerontologist, 1997. 37(2): p. 150-6.

40. $\quad$ Eckert, G.P., et al., Regulation of central nervous system cholesterol homeostasis by the liver $X$ receptor agonist TO-901317. Neurosci Lett, 2007. 423(1): p. 47-52.

41. Wahrle, S.E., et al., Overexpression of ABCA1 reduces amyloid deposition in the PDAPP mouse model of Alzheimer disease. J Clin Invest, 2008. 118(2): p. 671-82.

42. Zelcer, N. and P. Tontonoz, Liver X receptors as integrators of metabolic and inflammatory signaling. J Clin Invest, 2006. 116(3): p. 607-14.

43. Whitney, K.D., et al., Regulation of cholesterol homeostasis by the liver X receptors in the central nervous system. Mol Endocrinol, 2002. 16(6): p. 1378-85.

44. Li, G., et al., Statin therapy and risk of dementia in the elderly: a community-based prospective cohort study. Neurology, 2004. 63(9): p. 1624-8. 
45. Wrobel, J., et al., Indazole-based liver $X$ receptor (LXR) modulators with maintained atherosclerotic lesion reduction activity but diminished stimulation of hepatic triglyceride synthesis. J Med Chem, 2008. 51(22): p. 7161-8.

46. Yang, C., et al., Disruption of cholesterol homeostasis by plant sterols. J Clin Invest, 2004. 114(6): p. 813-22.

47. Plat, J., J.A. Nichols, and R.P. Mensink, Plant sterols and stanols: effects on mixed micellar composition and LXR (target gene) activation. J Lipid Res, 2005. 46(11): p. 2468-76.

48. Kaneko, E., et al., Induction of intestinal ATP-binding cassette transporters by a phytosterolderived liver X receptor agonist. J Biol Chem, 2003. 278(38): p. 36091-8.

49. Jansen, P.J., et al., Dietary plant sterols accumulate in the brain. Biochim Biophys Acta, 2006.

50. Hac-Wydro, K., et al., Cholesterol and phytosterols effect on sphingomyelin/phosphatidylcholine model membranes--thermodynamic analysis of the interactions in ternary monolayers. J Colloid Interface Sci, 2009. 329(2): p. 265-72.

51. $\mathrm{Xu}, \mathrm{X}$., et al., Effect of the structure of natural sterols and sphingolipids on the formation of ordered sphingolipid/sterol domains (rafts). Comparison of cholesterol to plant, fungal, and disease-associated sterols and comparison of sphingomyelin, cerebrosides, and ceramide. J Biol Chem, 2001. 276(36): p. 33540-6.

52. Ehehalt, R., et al., Amyloidogenic processing of the Alzheimer beta-amyloid precursor protein depends on lipid rafts. J Cell Biol, 2003. 160(1): p. 113-23.

53. Martin, S.W., B.J. Glover, and J.M. Davies, Lipid microdomains--plant membranes get organized. Trends Plant Sci, 2005. 10(6): p. 263-5.

54. Mongrand, S., et al., Lipid rafts in higher plant cells: purification and characterization of Triton X100-insoluble microdomains from tobacco plasma membrane. J Biol Chem, 2004. 279(35): p. 36277-86.

55. Rebolj, K., et al., Steroid structural requirements for interaction of ostreolysin, a lipid-raft binding cytolysin, with lipid monolayers and bilayers. Biochim Biophys Acta, 2006. 1758(10): p. 1662-70.

56. Clifton, P., Lowering cholesterol - a review on the role of plant sterols. Aust Fam Physician, 2009. 38(4): p. 218-21.

57. Plosch, T., et al., Sitosterolemia in ABC-transporter G5-deficient mice is aggravated on activation of the liver-X receptor. Gastroenterology, 2004. 126(1): p. 290-300.

58. Itoh, N., et al., Large-scale, multicenter study of cerebrospinal fluid tau protein phosphorylated at serine 199 for the antemortem diagnosis of Alzheimer's disease. Ann Neurol, 2001. 50(2): p. 1506.

59. Bowman, G.L., et al., Blood-brain barrier impairment in Alzheimer disease: stability and functional significance. Neurology, 2007. 68(21): p. 1809-14.

60. Fratiglioni, L., et al., Incidence of dementia and major subtypes in Europe: A collaborative study of population-based cohorts. Neurologic Diseases in the Elderly Research Group. Neurology, 2000. 54(11 Suppl 5): p. S10-5.

61. Hebert, L.E., et al., Age-specific incidence of Alzheimer's disease in a community population. Jama, 1995. 273(17): p. 1354-9.

62. Lobo, A., et al., Prevalence of dementia and major subtypes in Europe: A collaborative study of population-based cohorts. Neurologic Diseases in the Elderly Research Group. Neurology, 2000. 54(11 Suppl 5): p. S4-9.

63. Andreasen, N., et al., Evaluation of CSF biomarkers for axonal and neuronal degeneration, gliosis, and beta-amyloid metabolism in Alzheimer's disease. J Neurol Neurosurg Psychiatry, 2001. 71(4): p. 557-8.

64. Calpe-Berdiel, L., et al., Increased plasma levels of plant sterols and atherosclerosis: a controversial issue. Curr Atheroscler Rep, 2009. 11(5): p. 391-8.

65. Calpe-Berdiel, L., J.C. Escola-Gil, and F. Blanco-Vaca, New insights into the molecular actions of plant sterols and stanols in cholesterol metabolism. Atherosclerosis, 2008.

66. Weingartner, O., et al., Vascular effects of diet supplementation with plant sterols. J Am Coll Cardiol, 2008. 51(16): p. 1553-61. 



\section{Summary / Samenvatting}




\section{Summary}

Alzheimer's disease (AD) is the most common form of elderly dementia, leading to a high burden on caregivers and costs to society. Research on the causality and treatment of $\mathrm{AD}$ is therefore of high social and economic importance. Based on immunohistological hallmarks featuring post mortem diagnosis of $\mathrm{AD}$, several hypothesises concerning the causality of late onset $\mathrm{AD}$ were postulated. The rationale in this thesis is based on the amyloid- $\beta$ (A $\beta)$ hypothesis. The close association between the development and progression of $\mathrm{A} \beta$ dependent $\mathrm{AD}$ pathology and alterations in the cholesterol metabolism are extensively described in chapter I. The peripheral cholesterol metabolism is clearly separated form the cerebral cholesterol metabolism. Whereas the influence of plasma cholesterol concentrations is rather indirect, alterations in the brain cholesterol metabolism are described to be directly involved in the development and progression of AD. A detailed overview of the most important cerebral cholesterol regulatory mechanisms is provided in the general introduction, along with their relation to $\mathrm{AD}$. In addition, the exogenous cholesterol analogues, plant sterols, and the potential consequences on their cerebral accumulation are introduced.

In chapter II of the thesis, we questioned whether alterations in the cerebral cholesterol metabolism are cause or consequence of the amyloid pathology in the brain. We applied a unique AD mouse model, possessing a more physiological expression pattern of the involved APP gene, namely the APPSLxPS1mut mouse. Brains and serum of APPSLxPS1 mut mice and control littermates were examined at different ages: at the pre-plaque stage ( 9 months) versus the advanced pathology stage (21 months). Different parameters in the brain cholesterol metabolism were measured and compared: cholesterol, its precursors and metabolites, and genes involved in cholesterol synthesis, transport and metabolizing. We found age-related alterations in their brain sterol profiles and in the expression of cholesterol metabolism-related genes in comparison with wild-type mice. Concentrations of 24(S)-OHcholesterol and desmosterol were only modestly affected at 9 months, whereas they were significantly increased in 21 months old APPSLxPS1mut mice in comparison to their healthy littermates. In parallel, the liver $\mathrm{x}$ receptor (LXR) responsive genes, Apoc1 and Abcal displayed an increase in mRNA expression pattern. We therefore concluded that the alterations in the cerebral cholesterol metabolism are rather a consequence of the AD pathology in the brains of APPSLxPS1mut mice. We suggested that the increased cerebral cholesterol 
turnover attempts to rescue and to cope with the cerebral AD pathology. Yet, we did not exclude a causative influence of an altered cerebral cholesterol metabolism in the development of Alzheimer's disease pathology.

In line with the outcome of the experiments obtained in chapter II, we fed aged APPSLxPS1mut mice a synthetic LXR agonist (T0901317) over 10 weeks in the terminal phase of the disease pathology (chapter III). We aimed to reverse/prevent $\mathrm{A} \beta$ related $\mathrm{AD}$ pathology and the parallel cognitive deficits. Upon this long term LXR activation, cognitive performances were significantly improved compared to the untreated APPSLxPS1mut mice. Surprisingly, in contrast to our hypothesis, A $\beta$ deposition was not reduced upon LXR activation. Our data suggest that rather an enhanced cholesterol flux is underlying the beneficial effects of T0901317. Cerebral cholesterol turnover may therefore be a suitable target in the treatment and/or retardation of neurodegenerative diseases, including those other than AD.

Several plant sterols, exclusively derivable form dietary origin are classified as exogenous, naturally occurring LXR agonists. Chapter IV deals with the consequences of the recently described accumulation of plant sterol within mice brains. In this chapter, we questioned the fate and the reversibility of cerebral plant sterol accumulation in mice brains. In addition, we investigated the capacity of the most prevalent plant sterols (sitosterol, campesterol and stigmasterol) to modulate $\mathrm{A} \beta$ production in an in vitro model for $\mathrm{AD}$. We demonstrated that 6 weeks of dietary enrichment with plant sterols resulted in an irreversible accumulation of plant sterols in the brains of normal mice, despite a drastic reduction of circulating plant sterol level upon 6 subsequent months. Moreover we found that campesterol is more efficiently transferred across an in vitro blood-brain barrier (BBB) model compared to sitosterol. Behind the $\mathrm{BBB}$, plant sterols are incorporated within the cholesterol rich lipid raft membrane domains of brain cells. A neuronal cell line stably over-expressing the amyloid precursor protein (APP), displayed a reduced processing of APP upon sitosterol administration. Together, these data pointed to the potential therapeutical implications for plant sterol enriched food spreads in the treatment and prevention of $\mathrm{AD}$.

In chapter $\mathbf{V}$, we investigated the functional consequences of cerebral plant sterol accumulation in mice. We investigated cognition, anxiety and motor skills in ATP binding-cassette transporter (Abc)g5 knockout mice, bearing strongly increased brain plant sterol concentrations. Except for an increased swimming speed in the Morris water escape maze, none of the above mentioned brain functions were influenced by the increased cerebral accumulation of plant sterols. However, 
whether plant sterols can modify cognitive decline in a model for AD remains to be elucidated.

In chapter VI, we hypothesized, based on the solely exogenous nature of plant sterols in the CSF and the impaired choroid plexus function in AD, that plant sterol concentrations in CSF are reduced in AD pathology and therefore can be added to the relevant spectrum of biomarkers for $\mathrm{AD}$. We found that, despite comparable plasma plant sterol concentrations, absolute sitosterol and brassicasterol concentrations were significantly reduced in cerebrospinal fluid (CSF) of AD patients. After correction for CSF cholesterol concentrations, only brassicasterol was found to be a relevant biomarker for $\mathrm{AD}$.

Together, our findings provide more insight into the role of cerebral cholesterol turnover in $\mathrm{AD}$. The main findings are described and reflected against the up to date literature in the general discussion (chapter VII). 


\section{Samenvatting}

Alzheimer's disease (AD), de meest voorkomende vorm van dementie in ouderen, leidt tot een hoge belasting op mantelzorgers en tot hoge kosten voor de samenleving. Onderzoek naar de oorzaken en behandeling van $\mathrm{AD}$ is dan ook van groot sociaal en economisch belang. Gebaseerd op immunohistologische kenmerken die de post mortem diagnose van $\mathrm{AD}$ kenmerken, zijn een aantal hypothesen over de causaliteit van "late onset" AD gepostuleerd. De naar voor gebrachte hypotheses van dit proefschrift zijn gebaseerd op de amyloid- $\beta$ (A $\beta$ ) hypothese. De nauwe relatie tussen de ontwikkeling en progressie van $A \beta-$ afhankelijk pathologie in $\mathrm{AD}$ en de daaraan gerelateerde veranderingen in het cholesterol metabolisme worden uitvoerig beschreven in hoofdstuk I. De perifere cholesterol huishouding is gescheiden van het cerebrale cholesterol metabolisme. De invloed van verandering van plasma cholesterol concentraties op de ontwikkeling van $\mathrm{AD}$ is eerder indirect. Echter, veranderingen in het cholesterol metabolisme in de hersenen is beschreven direct betrokken te zijn bij de ontwikkeling en progressie van $\mathrm{AD}$. In de algemene inleiding wordt een gedetailleerd overzicht van de belangrijkste cholesterol regulatiemechanismen in de hersenen in relatie tot AD gegeven. Verder worden ook de exogene cholesterolanalogen, namelijk de plantensterolen, en de mogelijke gevolgen van hun accumulatie in de hersen beschreven.

In hoofdstuk II van dit proefschrift stelden we de vraag of veranderingen in het cerebrale cholesterol metabolisme eerder oorzaak of gevolg zijn van de amyloid pathologie in de hersenen. We gebruikten hiervoor een uniek AD muis model, dat een meer fysiologisch expressie patroon van het betrokken APP-gen vertoont, namelijk de APPSLxPS1mut muis. Hersenen en serum van APPSLxPS1mut en controle muizen werden onderzocht op verschillende leeftijden: (1) in het preplaque stadium (9 maanden) en (2) in het eindstadium van de pathologie (21 maanden). Verschillende parameters van het cholesterol metabolisme in de hersenen werden gemeten en vergeleken: precursoren van cholesterol, cholesterol zelf, cholesterol metabolieten en genen die betrokken zijn bij cholesterol synthese, transport en de stofwisseling. In vergelijking met wild-type muizen vonden we leeftijds gerelateerde veranderingen in de sterol profielen in de hersenen en in de expressie van de cholesterol metabolisme-gerelateerde genen. Concentraties van 24 (S)-OHcholesterol en desmosterol werden in bescheiden mate beïnvloed op 9 maanden, terwijl zij significant verhoogd waren in 21 maanden oude 
APPSLxPS1mut muizen in vergelijking met hun niet-AD nestgenoten. Parallel hieraan was er een verhoogde transcriptie van de liver $\mathrm{X}$ receptor (LXR) responsieve genen, Apoc1 en Abca1. Daarom concludeerde we dat de veranderingen in het cerebrale cholesterol metabolisme een gevolg konden zijn van de AD-pathologie in de hersenen van APPSLxPS1mut muizen. We suggereerden daarom dat de verhoogde turnover van het cerebrale cholesterol dient om de hersenen te redden van de cerebrale amyloid pathologie in AD. Desondanks sluiten we niet uit dat veranderingen in het cholesterol metabolisme in de hersenen de ontwikkeling van $\mathrm{AD}$ mede kan veroorzaken.

Gebasseerd op de resultaten verkregen in hoofdstuk II, besloten we oude APPSLxPS1mut muizen 10 weken lang met een synthetische LXR agonist (T0901317) in de terminale fase van hun AD pathologie te behandelen (hoofdstuk III). Ons doel was de $\mathrm{A} \beta$ gerelateerde pathologie en de daaraan verbonden cognitieve achteruitgang om te keren of te voorkomen. Volgend op de langedurige LXR activatie werden de cognitieve prestaties aanzienlijk verbeterd in vergelijking met de onbehandelde APPSLxPS1 muizen. In tegenstelling tot onze hypothese was de $A \beta$ depositie niet verminderd na LXR activering. Onze gegevens suggereren dat een verbeterde cholesterol flux meer waarschijnlijk ten grondslag ligt aan de gunstige effecten van T0901317. Cerebrale cholesterol turnover kan dus een geschikt doelwit voor de behandeling en vertraging van neurodegeneratieve ziekten in het algemeen worden.

Verschillende plantensterolen zijn geclassificeerd als exogene, natuurlijk voorkomende LXR agonisten. Hoofdstuk IV behandelt de gevolgen van de onlangs beschreven accumulatie van plantensterolen in muizen hersenen. In dit hoofdstuk, stelden we het lot en de omkeerbaarheid van cerebrale plantensterolen accumulatie in muizen hersenen in vraag. Daarnaast onderzochten we de mogelijkheid van de meest voorkomende plant sterolen (sitosterol, campesterol en stigmasterol) om A $\beta$ productie te moduleren in een in vitro model voor AD. We hebben aangetoond dat 6 weken van dieet verrijking met plantensterolen resulteerde in een onomkeerbare ophoping van plantensterolen in de hersenen van normale muizen, ondanks een verdere afname van serum plantensterol concentraties bekomen door een plantenstanolen verrijkt dieet gedurende 6 daarop volgende maanden. Bovendien vonden we dat campesterol in vergelijking met sitosterol efficiënter over de bloedhersenbarrière (BHB) wordt getransporteerd. Achter de BBB worden plantensterolen opgenomen in de cholesterolrijke" lipid raft" membraan domeinen van hersencellen. Een neuronale cellijn die het amyloid precursor proteïne (APP) stabiel tot overexpressie brengt, toont een verminderde productie van $A \beta$ na 
toediening van sitosterol. Samen duiden deze gegevens op potentiële therapeutische implicaties voor plantensterolen verrijkte voedingsmiddelen in de preventie en behandeling van AD.

In hoofdstuk $\mathbf{V}$, onderzochten we de functionele gevolgen van de accumulatie van plantensterolen in muizen hersenen. We onderzochten cognitie, angst en motorische vaardigheden in de ATP-binding cassette transporter (Abc)g5 knock-out muizen. Abcg5 knock-out muizen vertonen sterk verhoogde concentraties aan plantensterolen in het plasma en brein. Uitgezonderd de hogere zwemsnelheid in de Morris water escape maze, werden geen van de hierboven genoemde functies van de hersenen beïnvloed door de toegenomen cerebrale accumulatie van plantensterolen. Echter, of plantensterolen de cognitieve achteruitgang in een model voor AD kan voorkomen of terugdringen moet nog worden opgehelderd.

Gebaseerd op de uitsluitend exogene aard van plantensterolen in de hersenen en de verminderde plexus choroideus functie in $\mathrm{AD}$, onderzochte wij in hoofdstuk VI de hypothese dat plantensterolen concentraties in liquor van $\mathrm{AD}$ patiënten lager liggen dan in controle patiënten en daardoor dus mogelijke biomarkers voor AD zijn. We vonden dat, ondanks vergelijkbare plasma concentraties aan plantensterolen, absolute sitosterol en brassicasterol concentraties aanzienlijk lager waren in de liquor van $\mathrm{AD}$ patiënten. Na correctie voor liquor cholesterol concentraties, vonden wij dat brassicasterol een een relevante biomarker voor $\mathrm{AD}$ is.

Tot slot bieden onze bevindingen meer inzicht in de rol van de cerebrale cholesterol turnover in de ontwikkeling en preventie van $\mathrm{AD}$. De belangrijkste bevindingen worden beschreven en gereflecteerd tegen de up to date literatuur in de algemene discussie (hoofdstuk VII). 



\section{Curriculum Vitae}




\section{Curriculum vitae}

Tim Vanmierlo was born on May $17^{\text {th }}, 1983$ in Neerpelt, Belgium. In 2001 he obtained his high school diploma at the Sint-Augustinusinstituut in Bree, Belgium. He started his bachelor education in molecular life sciences at the transnational University Limburg (tUL; campus Hasselt, Belgium) in 2002 and finalized this study in 2005 with great distinction. The minor and major internships of the bachelor study were performed at Maastricht University, respectively at the department of molecular genetics and at the department of molecular cell biology. Subsequently, he participated in the master "clinical molecular life sciences" at the tUL (campus Hasselt), with an internship at the department of molecular cell biology and at the department of neuroscience (Maastricht University) and graduated with great distinction in July 2006.

In October 2006, he started his $\mathrm{PhD}$ program as a Marie Curie fellow at the department of Neuroscience within the school for Mental Health and Neuroscience, member of the European graduate school of neuroscience (Euron) under supervision of Dr. M. Mulder and Prof. Dr. H. Steinbusch. The topic of his research was the modulation of the brain sterol metabolism in Alzheimer's disease. In October 2007, he went to Columbia University, New York, concerning a 3 months internship on the role of cerebral cholesterol receptors in Alzheimer's disease under supervision of Prof. Dr. A. Tall and Dr. N. Wang. From January 2008 till September 2010 he continued his PhD program, partly as a Marie Curie fellow, at the institute of clinical chemistry and clinical pharmacology at the University Clinics of Bonn (Germany), supervised by Prof. Dr. Dr. D. Lütjohann. Additionally, he performed a large part of his research in and in collaboration with laboratories at University Medical Centrum Groningen, Erasmus MC Rotterdam, Vrije Universiteit Amsterdam, St.-Radboud University Nijmegen, and University Clinics Homburg/Saar. During the $\mathrm{PhD}$ program, he actively attend international conferences, among which the Endo-Neuro-Phsycho meeting, the Alzheimer's disease / Parkinson's disease meeting, the Society of Neuroscience meeting and the European Lipoprotein Club. He obtained the first price for best oral presentation at the $13^{\text {th }}$ Euron PhD days in Nijmegen 2009. 


\section{List of publications}

- Vanmierlo T., Bloks V.W., van Vark-van der Zee L.C., Rutten K., Kerksiek A., Friedrichs S., Sijbrands E., Steinbusch H.W., Kuipers F., Lutjohann D. and Mulder M.: Alterations in brain cholesterol metabolism in the APPSLxPS1mut mouse, a model for Alzheimer's disease. J Alzheimers Dis. 2010 Jan;19(1):117-27

- Vanmierlo T., Rutten K., Dederen J., Bloks V.W., van Vark-van der Zee L.C., Kuipers F., Kiliaan A., Blokland A., Sijbrands E.J., Steinbusch H., Prickaerts J, Lütjohann D. and Mulder M.: Liver X receptor activation restores memory in aged $\mathrm{AD}$ mice without reducing amyloid. Neurobiol Aging. 2009 Aug 10 [Epub ahead of print]

- Vanmierlo T., Berbée J.F.P, Abildayeva K, Blokland A, Jansen P.J., Lütjohann D, Gautier T., Sijbrands E., Ramaekers F.C.S., Kuipers F., Rensen P.C.N. and Mulder M.: Apolipoprotein CI Knock-Out Mice Display Impaired Memory Functions.

J. Alzheimers Dis. Conditionally Accepted

- Lütjohann D., Vanmierlo T. and Mulder M.: Chapter V: "Cholesterol trafficking in the brain" in Ehnholm C. (ed) Cellular lipid metabolism, Berlin: Springer Berlin Heidelberg 131-155 (2009)

- Vanmierlo T., Weingärtner O., van der Pol S., Tamboli I., Kerksiek A., Friedrichs S., Sijbrands E., Steinbusch H., Laufs U., Böhm M., de Vries H.E., Walter J., Mulder M., Lütjohann D.: Irreversible accumulation of plant sterols in the brain

Submitted

- Vanmierlo T., Rutten K., van Vark -van der Zee L.C., Friedrichs S., Bloks V.W., Blokland A., Ramaekers F.C.S., Prickaerts J., Steinbusch H., Kuipers F., Lütjohann D., Mulder M.: Memory and Anxiety Related Behavior in Abcg5-/Mice Bearing High Brain Plant Sterol Concentrations.

Submitted

- Vanmierlo T., Popp J., Kölsch H., Kerksiek A., Frank Jessen F., Maier W., Steinbusch H., Mulder M. and Lütjohann D.: The Plant Sterol Brassicasterol as Biomarker in Alzheimer's Disease Submitted

- Diane Jansen D., Janssen C.I.F., Vanmierlo T., Hooijmans C.R., Dederen P.J., van Rooij D., ZinnhardtB., Nobelen C.L.M., Janssen A-L., Hafkemeijer A., Mutsaers M.P.C, Doedée A.M.C., Kuipers A.A.M., Broersen L.M., Mulder M. and Kiliaan A.J.: Compensatory mechanism for impaired synaptic function in aging Alzheimer mouse brains In preparation 
- Wang N., Yvan-Charvet L., Lütjohann D., Mulder M., Vanmierlo T., Kim T.W., Tall A.R..: ATP-binding cassette transporters G1 and G4 mediate cholesterol and desmosterol efflux to HDL and regulate sterol accumulation in the brain. FASEB J. 2008 Apr;22(4):1073-82.

- Mulder M. and Vanmierlo T.: "Cholesterol: nieuw therapeutisch target bij ziekte van Alzheimer" Neuropraxis, vol 11, 2007

- Jansen P.J., Lüthjohann D., Abildayeva K., Vanmierlo T., Plösch T., Plat J., von Bergmann K., Groen A.K., Ramaekers F.C.S., Kuipers F., Mulder M.: Dietary plant sterols accumulate in the brain.

Biochim Biophys Acta. 2006 Apr;1761(4):445-53

\section{Oral presentations}

- "Plant sterols in the brain, good or bad?" 1st Marie-Curie Fellow meeting (Maastricht, NL), 23-Feb-07

- "Liver X Receptor activation in an Alzheimer mouse model", 6th Dutch EndoNeuro-Psycho Meeting (Doorwerth, NL), 5-8-Jun-07

- “Liver X Receptor activation in an Alzheimer's, Disease mouse model improves memory deficits", 30th annual European Lipoprotein Club meeting (Tutzing, D), 3-6-Sep-07

- 'Liver X Receptor activation in an Alzheimer's, Disease mouse model improves memory deficits", 11th Euron PhD-student meeting (Brussel, B), 13-14-Sep-07

- 'Liver X Receptor activation in an Alzheimer's Disease mouse model improves memory deficits", 2nd Marie-Curie Fellow meeting (Maastricht, NL), 22-Feb-08

- "Plant sterols in the brain, good or bad?", 3rd Marie-Curie Fellow meeting (Maastricht, NL), 25-Mar-09

- "Plant Sterols in the Brain - Modulating Alzheimer's Disease Pathology", Nederlandse lipoprotein club NLC (Leiden, NL), 20-May-09

- "Plant Sterols in the Brain - Modulating Alzheimer's Disease Pathology", 13th Euron PhD-student meeting (First price oral presentation) (Nijmegen, NL), 1-2Oct-09

- "Plant Sterols in the Brain - Modulating Alzheimer's Disease Pathology", 39e Jahrestagung des Arbeitskreises "Klinischer Lipidstoffwechsel" (Maikammer, D), 26-28-Nov-09

- "Plant sterols crossing the blood brain barrier?", 4th Marie-Curie meeting (Maastricht, NL), 16-Mar-10 


\section{Poster presentations}

- "Liver X Receptor activation in an Alzheimer mouse model", 6th Dutch EndoNeuro-Psycho Meeting (Doorwerth, NL), 5-8-Jun-07

- "Liver X Receptor activation in an Alzheimer's Disease mouse model improves memory deficits", 7th institutes day School for Mental Health and Neuroscience (Maastricht, NL), 11-Apr-08

- "Plant sterols in the brain, good or bad?", 49th International Conference on the bioscience of lipids (ICBL) (Maastricht, NL), 26-30-Aug-08

- "Plant sterols in the brain, good or bad?", 12th Euron PhD-student meeting (Aachen, D), 18-19-Sept-08

- "Plant sterols in the brain, good or bad?", ADPD meeting (Praque, CZE), 1115-March-09

- "Plant sterols in the brain, good or bad?", 32nd annual European Lipoprotein Club meeting (Tutzing, D), 7-10-Sep-09

- "Plant Sterols in the Brain - Modulating Alzheimer's Disease Pathology", 9th institutes day School for Mental Health and Neuroscience (Maastricht, NL), 17Mar-10 



\section{Dankwoord}


$\mathrm{Na}$ bijna vier jaar hard werken, maar ook veel plezier en mooie ervaringen is mijn proefschrift eindelijk klaar. Ik noem het wel "mijn" proefschrift, maar het is natuurlijk een project dat tot stand gekomen is met de hulp, toewijding en steun van vele mensen. Daarom zou ik ook graag in dit deel van mijn proefschrift iedereen, al dan niet bij naam, willen bedanken die mij in de afgelopen vier jaren op welke manier dan ook in of naast het lab gesteund of geholpen heeft. Hierbij zou ik op de eerste plaats mijn begeleidingsteam willen danken voor opportuniteit om als Marie Curie fellow dit veelzijdige project te hebben mogen aanvatten.

Monique, dank $u$ wel omdat $u$ er als begeleider van mijn traject altijd voor me was. $\mathrm{Na}$ een geslaagde bachelor en master stage onder uw toezicht was ik gebeten door de planten sterolen en "Alzheimer's disease" microbes. Ik was dan ook erg blij dat $\mathrm{u}$ me de kans gaf om in dit vakgebied verder te gaan voor mijn doctoraatsonderzoek. In het eerste jaar in Maastricht was u mijn directe supervisor, maar ik apprecieer ook erg dat $\mathrm{u}$ mij ook nadien nauwgezet en met veel overgave bent blijven begeleiden. De afgelopen vier jaar heb ik veel van u geleerd. U hebt van mij een betere wetenschapper gemaakt en mij vele opportuniteiten gegeven. Ik weet dat ik niet altijd even stuurbaar was, maar $\mathrm{u}$ wist mij toch steeds met de nodige vrijheid en vertrouwen de juiste richting in te krijgen. In bijzonder bewonder ik uw brede, creatieve onderzoeks- en levensvisie, uw netwerk capaciteiten en uw luisterend oor. Verder wil ik u nog bedanken voor uw enorme gastvrijheid. Bedankt voor alles en ik hoop dat we in de toekomst nog verder samen kunnen werken.

Professor Dr. Dr. Dieter Lütjohann, I would like to thank you for your unconditional support and confidence in me. You gave me the opportunity to integrate in your nice Bonner "lipid-team". I can look back on many interesting lectures, courses and meetings where I had the opportunity to take part in. Although I was a little bit shocked that I had to hold a lecture in German in Maikammer, I am now proud to have done it. I'm really grateful that you let me integrate in your professional network and that you provided top infrastructure which allowed me to perform my project in optimal conditions. The numerous scientific discussions at coffee time, paper discussions at sun set, and project planning with ice cream were unique! I appreciate that you were always there, willing to discuss and help. I admire your scientific fascination and you broad theoretic background! After having spent almost three fantastic years in Bonn, Bonn will remain in the hearts of me and my family. Danke schön Dieter!

Professor Dr. Harry Steinbusch, bedankt omdat u promotor was van mijn Ph.D. project! Ik apprecieer uw nauwgezette opvolging, organisatie en wetenschappelijke 
discussie van het project. Ik ben blij dat ik deel uit kon maken van Euron graduate school of neuroscience als Marie Curie fellow zowel in als buiten uw afdeling. Bedankt!

Nicole, $u$ was een rode draad doorheen de complexe organisatie en administratie van mijn doctoraatstraject. Ik waardeer uw zichtbare en onzichtbare bijdrage enorm. Zonder $\mathrm{u}$ had deze project constructie onmogelijk kunnen werken. Ik kan me dan ook volledig scharen achter de conclusie van de reacreditatie commissie in Amsterdam “de Ph.D. studenten zouden een Nicole op elke locale Euron afdeling wensen"! Ik ben u en Euron dankbaar en erkentelijk voor de organisatie van talrijke cursussen, Euron Ph.D. dagen, Marie Curie fellow meetings en de steun om andere wetenschappelijke congressen en cursussen bij te kunnen wonen.

Vervolgens zou ik ook mijn paranimfen Kristiaan en Frederik willen bedanken voor hun steun en wetenschappelijke bijdrage in de afgelopen vier jaar. Als student, leerde ik jullie kennen als "carpool Belgen in Maastricht". De chemie volgde snel en niet veel later zat ik braaf bij jullie op de achterbank. Via memorabele sluiproutes, desperate -maar daarom niet minder schitterende- omwegen over Luik, passeerden dagelijks tal van diepgaande wetenschappelijk en minder wetenschappelijke discussies de revue op weg van en naar Maastricht. Samen met jullie schitterende vrouwen en kinderen zijn we een hecht vrienden-team geworden. Frederik en Annelies, ik ben dan ook enorm fier dat ik peter mag worden van één van jullie aankomende tweeling kinderen. Kristiaan en Ellen, we kijken samen met jullie uit naar een gemeenschappelijke terugkeer naar Limburg! Volgens de statistieken is het in 2012 aan jullie:)!

Het eerste jaar van mijn onderzoek werkte ik op het department of neuroscience en department of molecular cell biology in Maastricht. Paula en Karly jullie hebben mij mijn eerste stappen helpen zetten in het praktisch wetenschappelijk onderzoek. Paula ik kijk nog regelmatig met een glimlach terug naar de lijst met Duitse lab termen in mijn eerst labjournaal die je voor me maakte bij mijn eerste bezoek aan Bonn.

Dr. Rutten, Kris, ik wil je bedanken voor de nauwe betrokkenheid bij mijn projecten en je kritisch oog in de gemeenschappelijke papers. De gedragsexperimenten die we samen uitvoerden met zowel witte als zwarte muizen waren onvergetelijk. Je hebt me ook wetenschappelijk beter leren schrijven. Ik hoop in de toekomst nog met jou samen te kunnen blijven werken! 
Verder, zou ik iedereen van de afdeling neuroscience in Maastricht die mij op een of andere manier geholpen heeft willen bedanken en in het bijzonder: Hellen, Marianne, Wiel en Jorike. Ook mijn kamergenoten Sofie, Marko, Mayenne en Marie-Lune hebben me hier een fantastische tijd bezorgd. Sofie, jouw gedrevenheid werkte erg inspirerend en kort overleg was vaak voldoende voor vruchtbare uitkomst. Marko, jij zorgde voor de vrolijke noot op de kamer en ons "fietstochtje" over Limburgse heuvels staat me nog vers in het geheugen!

Dr. Jos Prickaerts, de samenwerking met u en uw groep (Kris, Daniel, Jochen, Olga, Eva, Annerieke, Sven en Ayhan) was vruchtbaar en erg aangenaam! De teamgeest en de gedrevenheid binnen jullie hechte groep onderstrepen het eendrachtige doelgerichte onderzoek. Ik kijk er dan ook erg naar uit om een volgende stap te zetten in het wetenschappelijk onderzoek als deel van uw groep! De pitchers, de ponies en het verloren sjoepke droegen bij aan fantastische anti-stress momenten die ik samen met jullie kon beleven na de SONS meeting in San Diego, 2007.

Graag zou ik ook Professor Dr. Folkert Kuipers en Vincent Bloks van het Universitair Medisch Centrum Groningen willen bedanken. Folkert, bedankt voor uw wetenschappelijke en organisatorische bijdrage aan de verschillende samenlopende projecten, alsook de mogelijkheid die ik kreeg om onder de deskundige begeleiding van Vincent in uw lab Q-RT-PCR experimenten uit te voeren. Vincent, bedankt voor je hulp bij de Q-RT-PCR experimenten. Jouw snelle, weloverwogen, kritische, maar zeer bruikbare "comments" op manuscripten hielpen me steeds weer snel op weg naar een beter verhaal!

Dr. Arjan Blokland, bedankt voor uw eerlijke, kritische visie en zeer bruikbare opmerkingen in de opbouw van manuscripten en de planning van experimenten. Ik vond het erg aangenaam om met u samen te werken!

Verder dank aan Professor Dr. Frans Ramaekers en de onderzoeksgroep molecular cell biology voor de samenwerkingen in het begin van mijn project: Mieke, Helma, Wiel, Frederik, An, Monique, Marie-Hélène, Ronald, Sandra, Francine,... Ook op de afdeling moleculaire genetica kon ik steeds terecht met vragen of hulp bij experimenten. In het bijzonder wil ik hier Patrick, Veerle, Ronit, Monique, Kristiaan, Ellen, Arjen, Inge, Pieter en Mathijs bedanken.

Uit Nijmegen zou ik Dr. Amanda Kiliaan, Jos, Carlijn en Diane willen bedanken voor de aangename en succesvolle samenwerking. Het wetenschappelijke overleg binnen de driehoek Monique-Amand-Dieter lijkt me een goede formule. Hopelijk 
kan deze samenwerking nog verder bekroond worden. Jos, bedankt voor je deskundige uitleg en hulp bij de immunohistochemische kleuringen.

Uit Leiden wil ik Dr. Patrick Rensen en Dr. Jimmy Berbée bedanken voor de korte, maar zeer efficiënte samenwerking.

During the second year of my Ph.D. trajectory, I spent the first three months at Columbia University Medical Centre in New York. I am grateful to Professor Dr. Alan Tall and his research group for providing me the opportunity to experience the work in their laboratory. In particular, I would like to thank Dr. Nan Wang. Nan, although the period of my stay was relatively short, I learned a lot of laboratory skills from you. Moreover, you taught me how to interpret laboratory protocols between the lines. Marit, dank je wel voor de erg aangename tijd in en naast het lab in New York! Ik heb erg genoten van de uitstapjes en de rondleidingen! Hopelijk treffen we ons nog eens in New York of Nederland! Ik kijk er naar uit!

The larger part of my research has been performed in Bonn. I would like to thank my colleagues of the lipid-team. Anja, I enjoyed our discussions on scientific approaches, political views, language, sports, music and movies. I also appreciate your help in the lab and with the (re)construction of the GC/MS. Silvia, I really liked to work together with you and appreciated your helpfulness at all points. I also enjoyed our family meetings and I hope that we help Helder and Christian to keep on meeting. Constanze and Steffi I enjoyed our relaxing joint noon breaks! Herein, I learnt a lot about plants, cooking and salads! I wish you both al the best in your future scientific careers! Milka, I admire your scientific dedication. You are the only one I know, who will achieve to become both pharmacist, M.D. and Ph.D.! However, don't forget to plan and take enough C.O.T.!

I would like to thank professor dr. med. G. Hartmann, director of the institute for clinical chemistry and clinical pharmacology at the university clinics Bonn for providing the facilities, necessary to perform my research.

Verder zou ik Dr. Eric Sijbrands en zijn onderzoeksgroep aan het Erasmuc M.C. willen bedanken voor de samenwerking. Leonie en Jeanette, bedankt voor jullie hulp en gedrevenheid in de uitvoering van onze gemeenschappelijke experimenten. Ik vond het erg prettig om met jullie samen te werken! Jorie, bedankt voor de leuke samenwerking en nog veel succes met het afronden van je proefschrift. 
Ook zou ik Dr. Elga de Vries en Susanne van der Pol van de Vrije Universiteit Amsterdam willen bedanken voor de samenwerking in verband met de in vitro bloed-hersen barrière.

Dear Professor Dr. Walter, I would like to thank you for the supporting discussions and the opportunity you gave me to join the AD journal clubs at your department. Irfan, thank you for the fruitful discussions and for the nice collaborations. I appreciated your help and input in my projects. I also enjoyed the time together with our families at the Waldau.

Katherine en Sheen, de link met de universiteit Hasselt, ik vond ons contact, hoewel meestal via mail, maar ook via euron/Marie Curie erg aangenaam en wie weet kunnen we in de toekomst nog verder samen werken!

Dr. Julius Popp, Dr. Heike Kölsch and Dr. Oliver Weingärtner, thank you for the nice collaborations.

Zowel in Bonn als in Maastricht hebben studenten bijgedragen aan het onderzoek. Daarom zou ik mijn dank willen uitdrukken aan Anke, Katrijn en Angelika. Ik wens jullie nog veel succes in jullie verdere carrière.

Buiten het lab ben ik ook mijn vrienden (Gert, Joris, Roel \& Liesbeth, Daphne \& Leander, Tom \& Ronny, An \& Pieter, Helen \& Luc en natuurlijk de meer dan fantastische mannen van ZVK Echternach) erg dankbaar voor de steun, de leuke ontspanning, het bezoek in Bonn en de gezellige avonden samen die bijgedragen hebben aan het welslagen van mij proefschrift.

Verder heb ik ook een schitterende familie rondom mij die me door dik en dun steunt en waar ik altijd bij de terecht kan. Mama en papa, bedankt voor de vele kansen die ik van jullie heb gekregen, het geloof in mijn kunnen en de onvoorwaardelijke steun. Naar jullie liefde, genegenheid en warm nest is het altijd fijn terug keren. Als het eens wat moeilijker gaat, denk ik aan mijn uurwerk,... tijd om gelukkig te zijn! Dank jullie wel! Bert, An en Louise, bedankt voor jullie steun! Bert, je bent de beste broer die ik me kan voorstellen en ik ben blij dat ik met om het even wat bij jou terecht kan! Veel succes nog in je opleiding en hopelijk heb je snel iets meer tijd! An, ik wens je nog veel succes toe met het afronden van je studie en hopelijk komen ook jullie ook snel weer dichterbij wonen! Louise, mijn peterke, doe het goed op school meisje! 
Willy en Myriam, tijdens dit proefschrift werden jullie officieel mijn schoonouders. Dit veranderde natuurlijk niet veel aan de goede band die we al hadden. Willy, ik bewonder je inzicht en je kunde op technisch, maar vooral op menselijk vlak. Zonder enige ervaring in het lab, kan ik met jou diepgaand van gedachten wisselen over experimenten en theorieën. Myriam, ik geniet van de band die je hebt met de kleinkinderen en ik ben er zeker van dat dit ook omgekeerd is! Ik apprecieer het erg dat jullie er steeds voor ons allen zijn! Daan, je bent de meest fantastische en sociale teenager die ik ken. Keep on the good work! Silke en Maarten, bedankt voor jullie steun en de mooie cover van dit boekje! De vele gezellige avonden met zijn allen rond de stookkorf en het stoom af laten op de hof waren de perfecte afleiding in drukke tijden.

Evy, Helder en Noortje, aan jullie heb ik alles te danken. Jullie wisten me steeds weer tot rust en tot lachen te brengen na een moeilijkere dag op het werk, maar jullie waren ook de eerste die een telefoontje kregen bij positieve resultaten. Helder, telkens jij vol enthousiasme op mij toeloopt als ik 's avonds thuis kom, straalt mijn hart en loopt mijn energie tank weer vol! Je bent een schitterend kereltje! Noortje, als ik nog maar aan je lief snoetje denk, komt er spontaan een brede glimlach op mijn gezicht! Ik hoop dat je mag opgroeien tot een flinke meid! Evy, de opofferingen die jij hebt gemaakt door met mij mee te verhuizen naar Duitsland, me naar New York te laten gaan, de zorg en liefde die je aan onze kinderen geeft en je onvoorwaardelijke begrip, steun en liefde waarmee je er dag na dag voor mij bent, zijn van onschatbare waarde. Ik ben je ongelooflijk dankbaar en ik ben dan ook ongelooflijk fier dat wij samen horen! Evy, jij bent een kei, want zonder jou had dit nooit kunnen lukken!

Het gevoel te hebben dat ik er nooit alleen voor heb gestaan, heb ik aan jullie allemaal te danken! 



\section{Abbreviations}




\section{Abbreviations}

18S rRNA 18 (Svedberg units) ribosomal RNA

aa amino acid

$\mathrm{ABC}$

ATP binding cassette

ACAT

Acyl CoA (Coenzyme A): Cholesterol Acyltransferase

$\mathrm{AD}$

Alzheimer's disease

AICD

amyloid precursor protein intracellular domain

APOE

apolipoprotein E

APP

$\mathrm{A} \beta$

amyloid precursor protein

BBB

amyloid $\beta$

BSA

$\mathrm{CA}$

blood-brain barrier

bovine serum albumine

$\mathrm{CB}$

cornu amonis

CHAPSO

cerebellum

ChAT

3-[(3-Cholamidopropyl)dimethylammonio]-1-propanesulfonate

CNS

$\mathrm{CoA}$

Choline Acetyltransferase

central nervous sytem

CSF

Co-enzyme A

CTX

cerebrospinal fluid

cortex

CYP27A1 cytochrome P450 Cholesterol 27-hydroxylase

CYP46A1

DAB-Ni

cytochrome P450 Cholesterol 24-hydroxylase

DG

Diaminobenzidine-Nickel

DHCR24

DM dendate gyrus

\section{DRM}

24-dehydrocholesterol reductase

distance moved

EDTA

detergent resitant membrane

EOAD

EZM

ethylenediaminetetraacetic acid

early onset Alzheimer's disease

FAD

elevated zero maze

FASN

familial Alzheimer's disease

FCS

Fatty Acid Synthase

FF-MAS

featal calf serum

FITC

Follicular Fluid Meiosis Activating Sterol

FTDP-17

Fluorescein isothiocyanate

Frontotemporal Dementia With Parkinsonism Linked to 
Chromosome 17

GC/MS gas chromatography / mass spectometry

GLM general linear model

GS-FID gas chromatography - flame ionization detector

HC hippocampus

HDL high density lipoprotein

HMGCR 3,3-hydroxymethylglutaryl Co-enzyme A Reductase

HMG-CoA 3,3-hydroxymethylglutaryl Co-enzyme A

IDL intermediate density lipoprotein

IHC immunohistochemical

IL1 interleukin 1

INSIG1 insulin induced gene 1

KPI Kunitz protease inhibitor

LCAT Lecithin-Cholesterol Acyltransferase

LDL low density lipoprotein

LDLR low density lipoprotein receptor

LOAD late onset Alzheimer's disease

LRP-1 low density lipoprotein receptor-related protein 1

LXR liver $X$ receptor

LXRE liver $X$ receptor responsive element

MMSE mini mental state examination

MRI Magnetic resonance imaging

mRNA messenger ribonucleotide acid

MS multiple sclerosis

MWEM Morris water escape maze

MWU Mann-Withney U

M $\beta C D \quad$ methyl $\beta$ cyclo dextrin

NADPH Nicotinamide Adenine Dinucleotide Phosphate Hydrogen

$\mathrm{NC}$ normal chow

NFT neurofibrillar tangles

NPC1L1 Niemann-Pick Disease, Type C 1 like 1

OF open field

OLT object location task

ORT object recognition task

PBS phosphate buffered saline

$\mathrm{PH} \quad$ post hoc

PHF paired helical filaments

PiB Pittsburg compound

PS1 presenilin-1 


$\begin{array}{ll}\text { PS2 } & \text { presenilin-2 } \\ \text { PSA } & \text { plant stanol enriched } \\ \text { PSE } & \text { plant sterol enriched } \\ \text { Q-RT-PCR } & \text { quantitative real time polymerase chain reaction } \\ \text { RXR } & \text { retinoid X receptor } \\ \text { SCAP } & \text { SREBP Cleavage activating protein } \\ \text { SD } & \text { standard deviation } \\ \text { SELADIN-1 } & \text { Selective Alzheimer's disease indicator 1 } \\ \text { SEM } & \text { standard error of the means } \\ \text { SREBP } & \text { Sterol Regulatory Element Binding Protein } \\ \text { TBS } & \text { tris buffered saline } \\ \text { TIZ } & \text { time in zone } \\ \text { T-MAS } & \text { testis meiosis-activating sterol } \\ \text { TMSi } & \text { Trimethylsilyl (gas chromatography) } \\ \text { TST } & \text { tail suspension task } \\ \text { VLDL } & \text { very low density lipoprotein } \\ \text { WST } & \text { Water Soluble Tetrazolium }\end{array}$



